\title{
Décrocher son diplôme (et l'emploi de ses rêves!)
}

Comment maîtriser les compétences essentielles menant au succès à l'école, au travail et dans la vie

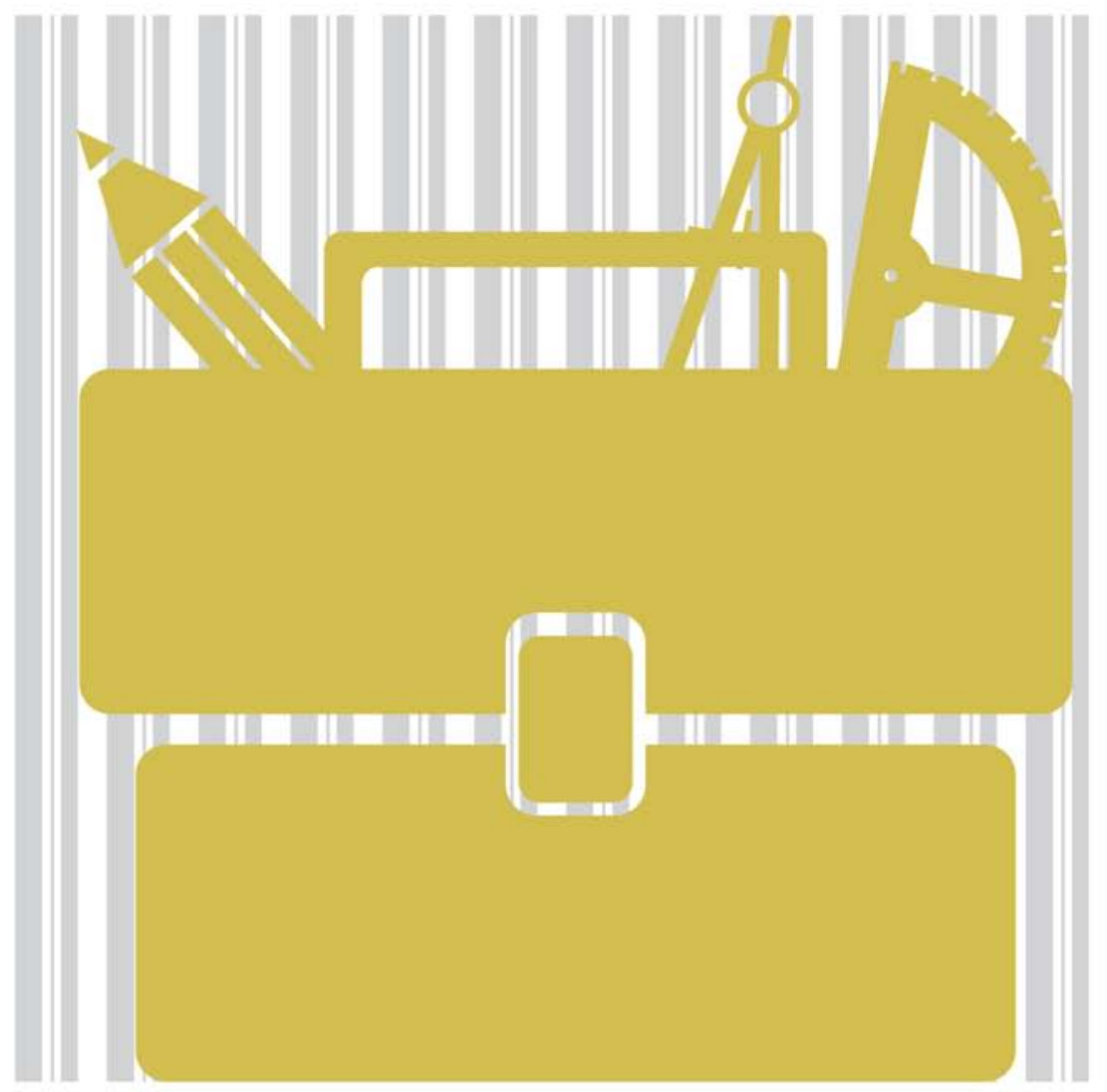

Thomas R. Klassen et John A. Dwyer Traduit par Émilie Laramée 


\section{DÉCROCHER SON DIPLÔME (ET L'EMPLOI DE SES RÊVES!)}


Page blanche conservée intentionnellement 


\section{DÉCROCHER SON DIPLÔME (ET L'EMPLOI DE SES RÊVES!) Comment maîtriser les compétences essentielles menant au succès à l'école, au travail et dans la vie}

Thomas R. Klassen et John A. Dwyer

Traduit par Émilie Laramée 
IIII Les Presses de I'Université d'Ottawa

University of Ottawa Press

Les Presses de l'Université d'Ottawa (PUO) sont fières d'être la plus ancienne maison d'édition universitaire francophone au Canada et le seul éditeur universitaire bilingue en Amérique du Nord. Depuis 1936, les PUO «enrichissent la vie intellectuelle et culturelle » en publiant, en français ou en anglais, des livres évalués par les pairs et primés dans le domaine des arts et lettres et des sciences sociales.

\section{Catalogage avant publication de Bibliothèque et Archives Canada}

Klassen, Thomas R. (Thomas Richard), 1957-

[How to succeed at university (and get a great job!). Français]

Décrocher son diplôme (et l'emploi de ses rêves!) /

Thomas R. Klassen et John A. Dwyer.

Traduction de: How to succeed at university (and get a great job!).

Comprend des références bibliographiques.

Publié en formats imprimé(s) et électronique(s).

ISBN 978-2-7603-2644-6 (couverture souple)

ISBN 978-2-7603-2646-0 (EPUB)

ISBN 978-2-7603-2647-7 (Kindle)

ISBN 978-2-7603-2645-3 (PDF)

1. Étudiants-Habiletés de base-Guides, manuels, etc. 2. Pensée critique. 3. Étude-Méthodes. 4. Succès. 5. Habiletés de base. I. Dwyer, John, 1948-, auteur II. Titre. III. Titre: How to succeed at university (and get a great job!). Français

Dépôt légal : premier trimestre 2018

Bibliothèque et Archives Canada

Bibliothèque et Archives nationales du Québec

(C) Presses de l'Université d'Ottawa 2018

Imprimé au Canada

Traduction : Émilie Laramée

Révision linguistique : Nicole Jetté

Correction d'épreuves: Karine Lavoie

Mise en page : Édiscript enr.

Maquette de la couverture : Édiscript enr.

Illustration de la couverture : iStock

Cet ouvrage est le fruit d'une collaboration entre Les Presses de l'Université d'Ottawa et l'École de traduction et d'interprétation de l'Université d'Ottawa.

Les PUO reconnaissent l'aide financière du gouvernement du Canada par l'entremise du Fonds du livre du Canada pour leurs activités d'édition. Elles reconnaissent également l'appui du Conseil des arts du Canada et de la Fédération canadienne des sciences humaines par l'intermédiaire des Prix d'auteurs pour l'édition savante. Nous reconnaissons également avec gratitude le soutien de l'Université d'Ottawa. 


\section{Table des matières}

Avant-propos

\section{Chapitre 1}

Se préparer à décrocher un bon emploi pendant les études

postsecondaires

À quoi servent les études postsecondaires ? ................................. 6

Que se passera-t-il une fois les études terminées ? ..................... 6

Y aura-t-il un emploi bien payé à la clé ?..................................... 9

Comment choisir mes cours .......................................................... 9

Étudiants adultes, retour aux études et études

à temps partiel ............................................................................... 10

Quelles compétences applicables seront acquises

pendant cette période?

\section{Chapitre 2}

Compétences pour réussir à l'école et au travail ................................ 15

Les présentations orales........................................................ 16

Les travaux de groupe................................................................ 24

Faire preuve $d$ 'initiative en tant que professionnel .................. 26

Savoir y faire avec les chiffres .................................................... 31

La prise de notes ...................................................................... 33

S'organiser............................................................................. 36

Se fixer des objectifs à long terme et des objectifs

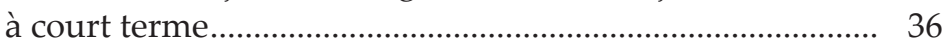

Gérer son temps .................................................................. 37

Garder le cap ...................................................................... 39

Gérer les distractions de manière efficace........................... 40

\section{Chapitre 3}

Réussir en classe et au travail............................................................ 43

Les examens, ça fait partie de la vie............................................. 44

Réduire l'anxiété ............................................................... 45

Se préparer pour un examen important ................................ 47

Le jour J .......................................................................... 48 
Stratégies pour réussir les examens à choix multiples .............. 49

Stratégies pour réussir les examens écrits.................................. 51

L'utilité des dissertations ......................................................... 56

Écrire pour un lectorat d'érudits................................................ 61

Comment organiser une dissertation............................................. 65

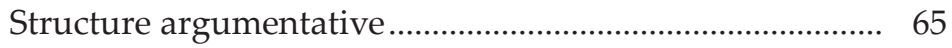

Structure explicative............................................................. 65

Structure descriptive .......................................................... 66

Structure comparative/exploratoire ……………….............. 66

Structure progressive ......................................................... 66

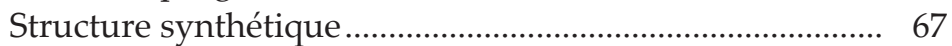

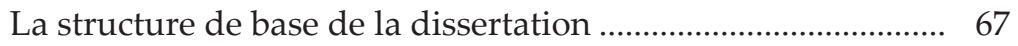

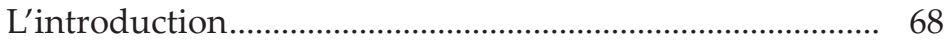

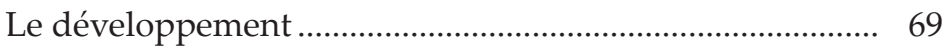

La conclusion.............................................................. 70

La rédaction de dissertations est un processus .......................... 72

La première ébauche ........................................................... 72

La deuxième ébauche ............................................................. 74

L'ébauche finale................................................................... 74

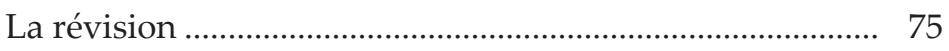

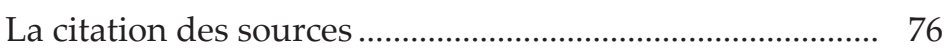

La page-titre....................................................................... 78

Les erreurs fréquentes en rédaction de dissertations ................ 78

La surutilisation de la voix passive .................................... 79

Le mélange de pronoms ..................................................... 79

Les erreurs de temps de verbes .......................................... 79

Les phrases alambiquées .................................................... 79

Les mauvais choix de mots ou les mots manquants........... 80

Les anglicismes ….............................................................. 80

L'accord du participe passé ................................................ 81

L'accord avec « on » ............................................................. 81

«Ça sonne correct ! » ........................................................... 81

Les rapports de laboratoire et autres devoirs du genre ............ 82

Le mot de la fin........................................................................ 82

\section{Chapitre 4}

Améliorer son sens critique ................................................................... 85

Qu'est-ce que le sens critique ?................................................. 86

Communication et pensée critique............................................... 87

Améliorer son sens critique....................................................... 89 
Les limites du récit.................................................................... 92

Mettre l'information en contexte .............................................. 94

La signification des théories ...................................................... 98

Niveaux et formes théoriques ....................................................... 100

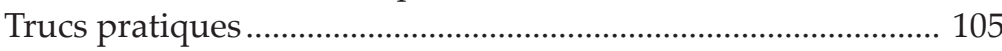

\section{Chapitre 5}

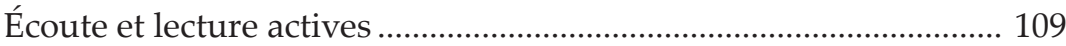

L'écoute passive................................................................ 110

L'écoute active ............................................................... 110

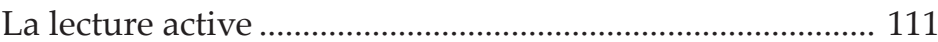

La lecture au niveau postsecondaire..................................... 112

Améliorer ses habiletés .............................................................. 116

\section{Chapitre 6}

Se documenter sur un sujet ................................................................. 119

Les règles de base de la recherche ................................................ 120

Deux stratégies de recherche...................................................... 124

L'écriture spontanée ………………………………............. 124

La carte mentale .................................................................. 125

\section{Chapitre 7}

La résolution de problèmes pratiques à l'école, au travail et dans la vie

Pourquoi la résolution de problèmes pratiques est-elle

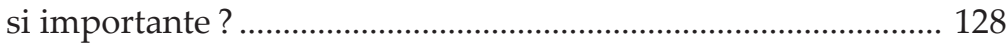

La résolution de problèmes comme processus.......................... 129

Étape 1 : définir le problème.......................................................... 132

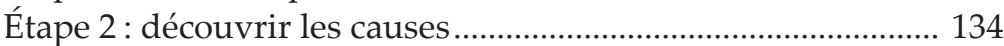

Étape 3: dresser la liste des solutions ......................................... 137

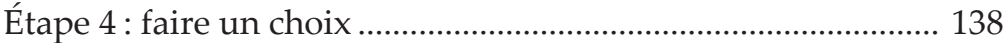

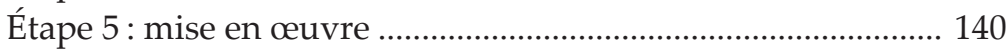

Étape 6 : points de repère et évaluation......................................... 141

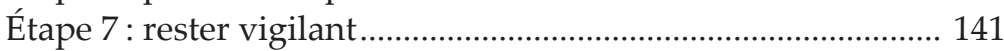

La résolution de problèmes comme compétence de vie ............ 142

\section{Chapitre 8}

Faire preuve de créativité en résolution de problèmes ..................... 143

Le lien avec la résolution de problèmes ........................................... 144

La rigidité de l'esprit.................................................................... 146 


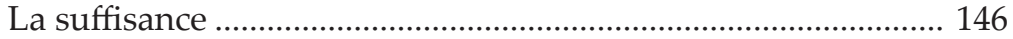

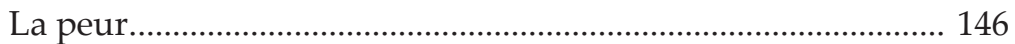

La cohérence/la constance .......................................................... 147

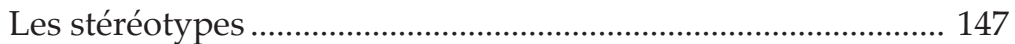

La compression/la réduction........................................................ 147

Techniques créatives de résolution de problèmes...................... 149

Configuration créative du problème.............................................. 153

Maximiser les solutions créatives ................................................. 154

L'environnement idéal pour penser de manière créative........... 155

\section{Chapitre 9}

Trouver et décrocher l'emploi idéal.................................................. 159

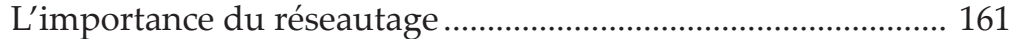

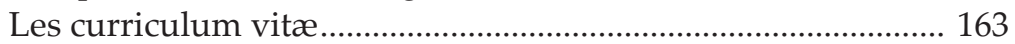

Exemples de curriculums vitæ ............................................ 165

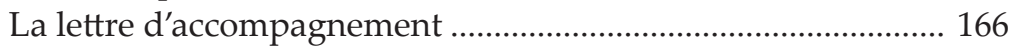

Format de la lettre d'accompagnement ............................... 168

Ce qu'il faut éviter ................................................................ 170

Le réseautage et l'entrevue d'information..................................... 172

Après l'entrevue d'information ............................................. 173

Le réseautage quand on est aux études................................. 174

L'usage inapproprié de l'entrevue d'information................ 176

Réseautage et mentorat.................................................... 176

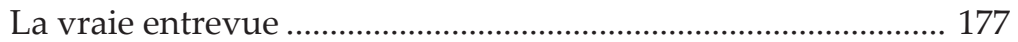

Les exceptions ..................................................................... 179

Le courriel ou la lettre de remerciement ............................... 180

Décoder les questions d'entrevue................................................ 180

Les services et les entrevues sur le campus ........................... 188

Les entrevues dans de petites entreprises............................. 188

Les styles d'entrevue ...................................................................... 189

L'approche conversationnelle................................................. 190

L'approche par consensus.................................................... 190

L'approche par stress ............................................................... 190

Les entrevues d'une journée et au restaurant....................... 191

Les deuxième et troisième entrevues................................... 191

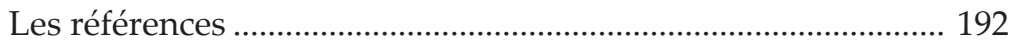

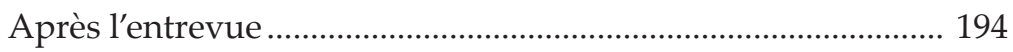

Annexe 1: Exemples de curriculum vitæ..................................... 195

Annexe 2: Exemple de lettre de présentation............................... 201

Annexe 3: Exemple de lettre de remerciement ........................... 203 


\section{Chapitre 10}

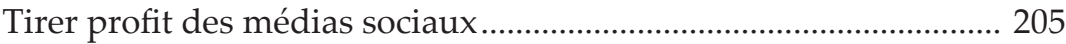

Les cinq règles d'or des médias sociaux ...................................... 206

Les médias sociaux et la création de relations............................ 208

Créer une image de marque ......................................................... 210

La métaphore des sites Web de rencontre.................................... 213

\section{Chapitre 11}

La réussite au travail et au-delà ........................................................... 215

Le premier emploi...................................................................... 216

Se garder à jour ............................................................................ 219

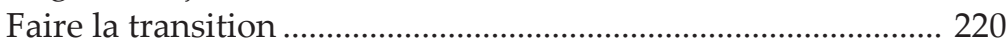

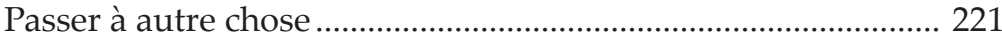

On n'arrête jamais d'apprendre ..................................................... 223

Les études supérieures, les formations professionnelles et techniques, et les cours en ligne ................................................ 223

Les collèges communautaires et la formation en ligne .............. 226

Cumuler les succès ..................................................................... 227

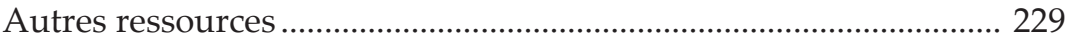

Au sujet des auteurs ............................................................................. 233 
Page blanche conservée intentionnellement 


\section{Avant-propos}

ous avons écrit ce livre pour répondre aux nombreuses ques-
tions que se posent les étudiants et les nouveaux diplômés sur la réussite à l'école et sur la carrière qui s'ensuivra. Nos conseils proviennent de questions que nous ont posées les milliers d'étudiants à qui nous avons enseigné aux universités Ryerson, Trent, de la Colombie-Britannique, Simon Fraser, York, ainsi qu'au North Island College, sur l'île de Vancouver, au Canada.

Ce livre est le résultat d'un effort collectif. Des étudiants et des collègues de l'Université York ont vivement insisté pour que nous en écrivions la première version, A Practical Guide to a Great Job after University. L'accueil chaleureux qu'il a reçu d'une grande variété de lecteurs (des parents, des conseillers pédagogiques, des conseillers d'orientation, mais, surtout, des étudiants) nous a motivés à en rédiger un deuxième, amélioré : Flourishing in University and Beyond.

Des centaines d'étudiants nous ont dit par courriel, en classe et lors de rencontres que le livre les avait aidés à trouver leur voie et qu'il avait même parfois changé leur vie. L’Université York et d'autres universités en ont fait un livre obligatoire pour quiconque entame ses études postsecondaires et le recommandent à tous ceux qui sont sur le point d'obtenir leur diplôme. Le livre a accumulé les critiques positives, dont celle du Globe and Mail. Le fait qu'autant de personnes nous ont dit avoir bénéficié de nos conseils et de nos trucs a achevé de nous convaincre d'en publier une troisième édition, revue et améliorée. 
C'est elle que vous tenez présentement entre vos mains ou que vous lisez à votre écran.

Cette édition contient du nouveau matériel sur l'écoute active, la prise de notes et les médias sociaux ainsi que des mises à jour de tous les chapitres. Au moment de rédiger cette nouvelle version, nous avons eu le privilège d'obtenir l'appui des Presses de l'Université de la Colombie-Britannique (UBC). Emily Andrew, rédactrice en chef à Toronto, a été l'une des premières à nous encourager. Sans son aide, le projet n'aurait jamais abouti. De la manière douce et efficace qui lui est propre, Melissa Pitts, directrice des Presses de l'Université de la Colombie-Britannique, s'est assurée que tous les éléments du livre étaient cohérents. Holly Kelly, directrice des services de la production et de la rédaction, a veillé, avec son équipe, à ce que le manuscrit passe de mots mal formés au charmant produit final qu'il est devenu.

Nous demeurons reconnaissants aux collègues actuels et anciens de l'Université York, Stephen Glassman, Michael Jackel et Michael Legris, d'avoir soutenu les premières versions du livre. Nous sommes aussi profondément reconnaissants envers tous nos collègues du monde universitaire et au-delà, qui nous ont encouragés à écrire un ouvrage qui combine études postsecondaires et compétences de vie, deux ensembles qui sont trop souvent séparés.

Dans cette édition, nous continuons à faire sauter les barrières entre l'expérience des études postsecondaires et les carrières après l'obtention du diplôme. Nous croyons toujours fermement que les compétences pour réussir aux études et celles pour le faire au travail sont similaires et s'apprennent. Nous savons que ceux qui adjoignent les bons conseils à la motivation finissent invariablement par s'épanouir à l'école et au-delà. 


\section{Se préparer à décrocher un bon emploi pendant les études postsecondaires}

«Le moment idéal est toujours le moment présent puisque lui seul permet de passer à l'action. » - Georges Vanier

$\mathrm{L}$ es études postsecondaires coûtent plus cher que jamais et sont de plus en plus exigeantes. De plus, et contrairement à ce qu'il en était il n'y a pas si longtemps, l'obtention d'un diplôme ne garantit pas qu'on trouvera un emploi, encore moins un bon emploi. La concurrence sur le marché du travail est sans merci, les bons emplois se font rares et les employeurs placent la barre très haut. Pour se trouver du travail, les nouveaux diplômés doivent non seulement rivaliser les uns avec les autres, mais souvent avec des candidats d'autres régions et pays.

Même si vous savez qu'il est important de faire des études postsecondaires pour obtenir un bon emploi, il n'est pas toujours évident pour vous de voir le lien entre études et travail. Plusieurs cours semblent en effet peu pertinents ou trop abstraits, et les professeurs peuvent paraître déconnectés de la réalité et de ses exigences. Shakespeare, Marx et Platon font clairement partie de leur top 10 des personnages importants, mais qu'en est-il de vos futurs employeurs? Plusieurs patrons et collègues plus âgés, en particulier au sein de petites entreprises, n'ont peut-être pas complété d'études supérieures 
et ne savent pas nécessairement à quel point il faut travailler dur pour obtenir un diplôme. Vous serez peut-être tenté de faire preuve de cynisme ou de découragement quand viendra le temps d'assister aux cours, de faire des recherches en bibliothèque, de rédiger des dissertations et de préparer des présentations et des examens.

Quand vous obtiendrez votre diplôme, vous aurez dépensé beaucoup d'argent pour ce petit bout de papier. En effet, les droits de scolarité de la première année d'études postsecondaires s'élèvent en moyenne à $6300 \${ }^{1}$. À ce montant, il faut ajouter le coût des livres et du matériel scolaire, les frais de transport ainsi que le loyer, si vous n'habitez pas chez vos parents. Au total, un diplôme d'études postsecondaires pourrait vous coûter au moins 30000 \$, et assurément plus si vous devez reprendre des cours ou si vous changez de programme d'études. Certains d'entre eux, comme les programmes en gestion et en sciences informatiques, coûteront plusieurs milliers de dollars supplémentaires en droits de scolarité chaque année. Si vous quittez la maison pour étudier, le coût de votre diplôme pourrait donc s'élever à $70000 \$$ !

Si vous obtenez un diplôme d'études postsecondaires, vous grossirez les rangs des 64 \% d'adultes canadiens qui ont fait de même, dont $25 \%$ détiennent un grade universitaire. Soutenu ou non par votre famille, vous aurez travaillé dur et fait beaucoup de sacrifices pour appartenir à ce groupe sélect. Malheureusement, détenir un diplôme ne garantit pas une carrière prometteuse.

Dans un monde parfait, votre emploi idéal vous attendrait dès votre cérémonie de collation des grades terminée. Illusoire? Pas du tout! Avec un peu de planification, cela pourrait très bien vous arriver. Il suffit de vous préparer intelligemment et stratégiquement pour le marché du travail et de transformer les compétences acquises pendant vos études en aptitudes que recherchent les employeurs !

Que vous veniez de commencer vos études postsecondaires, que vous vous trouviez au beau milieu de celles-ci ou que vous soyez sur le point d'obtenir votre diplôme, ce livre vous aidera à acquérir les compétences menant au succès à la fois dans vos études et sur l'exigeant marché du travail. Il s'adresse à tous les étudiants, qu'ils soient à l'université, au collège, au cégep ou au secondaire, ainsi qu'à leurs parents. Bien qu'il soit préférable de le lire avant d'entreprendre des études postsecondaires, ceux qui s'apprêtent à quitter le collège ou l'université pour commencer un emploi à temps plein y trouveront aussi leur compte. 
Ce livre vous permettra également de vous épanouir sur les plans scolaire, professionnel et personnel. S'épanouir signifie vous développer au point de vue personnel et accroître votre potentiel de contribution aux autres, y compris aux employeurs. Au bout du compte, il s'agit du bonheur qui accompagne une vie réfléchie, équilibrée et sur laquelle vous avez le contrôle. Ne vous laissez surtout pas intimider par la connotation sophistiquée de ces termes. S'épanouir est à la portée de tous.

Pourtant, nombreux sont ceux qui n'arrivent pas à s'épanouir, que ce soit dans les études ou au travail, car ils veulent à tout prix décrocher un emploi incroyable au salaire faramineux. Ils acceptent, sans la remettre en question, la pression que leur mettent parents, amis et autres personnes influentes dans leur vie. Les étudiants sont, en effet, constamment bombardés de messages subtils, mais puissants, qui visent à les diriger vers les professions traditionnellement prestigieuses, comme le droit, la médecine et la comptabilité, ou vers des cours et des programmes qui se marchandent mieux.

Vous risquez de ne pas vivre pleinement votre expérience des études postsecondaires - ni votre vie - si vous vous limitez de la sorte. Les études postsecondaires peuvent mener à un emploi épanouissant et bien payé, mais là n'est pas leur principale raison d'être.

Faire de vos études une simple et bête préparation à un emploi potentiel ne mènerait à rien, de toute façon. Personne ne peut prédire avec exactitude à quoi ressemblera le marché du travail après les quelque quatre années que vous prendrez généralement pour obtenir votre diplôme. Il est donc préférable de profiter pleinement de votre expérience des études postsecondaires et de ne pas vous inquiéter constamment de votre avenir. Il reste, toutefois, que ce serait faire preuve de gros bon sens que d'utiliser vos années d'études pour vous préparer à réussir dans la vie après avoir obtenu votre diplôme. Vous vous épanouirez d'autant plus dans vos études, au travail et dans la vie si vous portez une attention particulière à cette dynamique entre les études postsecondaires et le succès dans la vie.

En effet, si vous ne comprenez pas comment l'école est liée au travail et le travail, à l'école, vous ne tirerez pas pleinement avantage de vos études postsecondaires pour vous préparer au marché du travail. Décrocher un emploi ne doit pas vous occuper complètement l'esprit; en fait, nous vous montrerons comment le fait d'être obsédé par ce sujet peut se retourner contre vous. Il s'agit simplement de prendre en considération la connexion entre l'école et le travail. 
Plus vous saisirez la nature de ce lien, mieux vous serez préparé et moins vous serez stressé quant à votre avenir, sans compter que vous aurez beaucoup plus de plaisir à étudier!

\section{À quoi servent les études postsecondaires?}

«Toto, j'ai l'impression que nous ne sommes plus au Kansas. » Cette réplique classique du Magicien d'Oz résume l'expérience de bon nombre d'étudiants qui entreprennent des études postsecondaires, où les exigences sont plus élevées qu'au secondaire et où il est plus difficile d'obtenir de bons résultats. Pour couronner le tout, la transition sociale et culturelle de l'école secondaire au collège ou à l'université ajoute à tout un coefficient supérieur de difficulté.

Les études postsecondaires servent à vous préparer non seulement à votre emploi, mais aussi au reste de votre vie. Grâce à elles, vous découvrirez des matières dont vous n'aviez jamais entendu parler et dont vous ignoriez donc qu'elles vous intéresseraient. Ces études englobent plusieurs choses: apprendre, rencontrer des gens, prendre des décisions, tisser des liens avec autrui, peut-être quitter la maison, voyager, faire face à des difficultés financières, travailler à temps partiel, voire à temps plein, et plus encore.

Vous vous ferez de nouveaux amis et rencontrerez peut-être l'amour de votre vie. Vous en apprendrez très certainement beaucoup sur l'amour, la vie et le domaine d'études que vous avez choisi, mais une fois votre diplôme en poche, une bonne partie de cet apprentissage semblera très peu pertinent à votre emploi et à votre carrière. Pourtant, le temps que vous passerez aux études pourrait - et devrait être consacré à préparer votre entrée sur le marché du travail.

Comme nous l'expliquons dans ce livre, il n'y a rien de mieux que les études postsecondaires pour découvrir et cultiver ses ambitions de carrière. Ce qu'il y a de merveilleux, c'est que vous pouvez à la fois profiter autant que vous le voulez de vos années d'études et vous assurer de décrocher l'emploi de vos rêves après l'obtention de votre diplôme. Trop beau pour être vrai ? Poursuivez votre lecture pour le savoir!

\section{Que se passera-t-il une fois les études terminées?}

Une des plus grandes inquiétudes qui accompagnent les études postsecondaires est de ne pas savoir ce qui arrivera après. Vais-je me 
trouver un bon emploi ? Vais-je l'aimer? Gagnerai-je assez d'argent? Devrai-je déménager pour trouver du travail ? Serai-je coincé dans un rythme de vie effréné sans avoir le temps de reprendre mon souffle? Me retrouverai-je au chômage? Ces questions sont d'une importance capitale. Heureusement, il est possible de répondre à la majorité d'entre elles dès maintenant.

Quand vous obtiendrez votre diplôme, vous intégrerez un marché professionnel différent de celui de vos parents ou, s'ils ont fait des études postsecondaires, de celui avec lequel ils sont familiers. Il n'y a pas si longtemps, détenir un diplôme d'études postsecondaires garantissait l'obtention d'un bon poste permanent. De nos jours, les entreprises exigent plus qu'un diplôme : elles cherchent des personnes qui font preuve de flexibilité, peuvent régler des problèmes, sont polyvalentes, s'adaptent bien au changement et travaillent bien en équipe.

Vos parents ne comprendront pas nécessairement à quel genre de pression vous êtes exposé, étant donné qu'ils n'auront peut-être pas eu la même expérience que vous. Ce qui les préoccupe, c'est que vous trouviez un poste stable et que vous subveniez à vos besoins le plus rapidement possible. Comme vos professeurs, ils ont tendance à croire que les choses n'ont pas vraiment changé depuis le temps où ils étaient jeunes. Il n'est parfois pas inutile de leur rappeler ce qu'ils faisaient à votre âge.

Pour être réellement en mesure de subvenir à vos besoins et de vous épanouir pleinement, vous devrez faire preuve de beaucoup plus d'initiative et de flexibilité qu'ils l'ont fait à votre âge. Votre cheminement de carrière sera parsemé d'embûches, ce qui ne signifie pas nécessairement qu'il sera stressant, et certainement pas moins intéressant. Cela signifie tout simplement que vous ne pouvez pas vous permettre d'être passif : vous devez être stratégique, créatif et saisir les occasions qui se présentent à vous.

Après trois ou quatre ans à étudier comme un forcené et à tirer le diable par la queue, il est normal d'espérer être récompensé par un emploi permanent et stable. Il est, par contre, très peu probable que cela se produise. En effet, ce n'est pas la façon dont le marché du travail fonctionne. La plupart des employeurs offrent généralement des contrats à court terme aux nouveaux diplômés de collèges et d'universités. Il s'agit là d'une façon pour chacun de se mettre au banc d'essai : le courant passe-t-il ? Le nouvel employé veut-il et peut-il apprendre?

De nos jours, les employeurs des secteurs privé et public ne cherchent pas des employés qui demeureront pour toujours au sein 
de leur organisation. Ils s'attendent, en effet, à ce que les gens restent à l'affût de nouvelles occasions de carrière et fassent le saut aux cinq ans environ. Autrement dit, les employeurs s'attendent à ce qu'un bon pourcentage de leurs employés actuels quitte pour un autre emploi, à la recherche de nouveaux défis et d'un meilleur salaire. Demeurer trop longtemps au même endroit pourrait même être perçu comme un manque d'ambition! C'est là la réalité du monde du travail qui sera la vôtre.

Un professionnel doit être prêt à apprendre sa vie durant, à continuellement se mettre à niveau et à développer de nouvelles compétences. Apprendre n'est plus circonscrit à une seule période dans la vie d'un individu; c'est un processus continu. Chacun a la responsabilité de se garder à jour et de suivre les développements dans son domaine ou dans la carrière qui l'intéresse, et celui qui demeure passif ou qui ne prend pas d'initiatives finit par mordre la poussière.

Étant donné l'importance qu'aura l'apprentissage dans votre vie professionnelle future, il est logique de commencer à apprendre alors que vous êtes aux études. La plupart des cours postsecondaires vous donneront l'occasion de mettre à l'essai les compétences qui vous permettront de vous adapter à votre milieu de travail et au-delà. Après tout, vous aurez aussi une carrière à gérer! Vos années d'études postsecondaires sont pour vous une occasion unique d'apprendre, de mettre en pratique et de perfectionner les compétences essentielles au succès sur le marché du travail.

En tant que futur professionnel, vous aurez besoin de quatre ensembles de compétences:

- excellentes habiletés en communication (lecture, écriture, expression orale, écoute) ;

- facilité à apprendre et à résoudre des problèmes (autrement dit, la capacité à penser de façon critique, créative et offrant une «valeur ajoutée »);

- capacité à travailler en équipe et à vous comporter avec les autres (éthique, attitude positive, responsabilité) ;

- volonté de s'adapter aux circonstances changeantes et d'appliquer ses connaissances à des situations nouvelles.

Plus vous maîtriserez ces compétences, mieux vous serez préparé à contribuer à votre profession et à atteindre vos objectifs de carrière. En plus, devinez quoi : ces compétences constituent aussi, ni plus ni moins, la clé du succès des études postsecondaires. Ce sont ces compétences dont nous traitons dans ce livre. 


\section{Y aura-t-il un emploi bien payé à la clé?}

Les bons emplois sont habituellement accompagnés de bons salaires et la majorité des diplômés universitaires et collégiaux finissent par se trouver un poste bien rémunéré. Votre but est de faire comme eux et d'éviter de rester coincé dans un travail mal payé qui ne vous satisfait pas.

L'argent ne fait pas le bonheur, mais en avoir n'y nuit pas. Pour plusieurs diplômés, avoir un bon revenu est une nécessité, puisqu'ils sont criblés de dettes. Rembourser vos prêts et avoir, peut-être, de l'argent pour voyager, acheter les choses dont vous vous êtes privé, voire vous marier, sont de bonnes raisons de prendre au sérieux la préparation à votre carrière et votre recherche d'emploi.

Vous serez probablement heureux d'apprendre que trois ans après avoir terminé un baccalauréat, votre salaire s'élèvera à environ $41600 \${ }^{2}$. Il s'agit là, bien sûr, du salaire moyen; certains diplômés gagneront considérablement plus, alors que d'autres feront moins. Le but, c'est de faire partie de ceux qui gagnent plus et qui, critère tout aussi important, aiment ce qu'ils font!

\section{Comment choisir mes cours}

Un des aspects les plus stressants des études postsecondaires est de devoir choisir un programme, une majeure ou une spécialisation, et des cours. Vous recevrez certainement moult conseils d'orienteurs, de conseillers aux études, de professeurs, de membres de votre famille et d'amis, mais, au final, c'est vous qui devrez décider. Et c'est une décision importante. Bien sûr, certains programmes ou cours sont peut-être hors de votre portée à cause de leurs exigences et de leurs préalables, mais vous aurez tout de même une foule de choix à faire. Voici le meilleur conseil de ce livre: faites ces choix pour vous et pas pour quelqu'un d'autre!

Faites-vous confiance. Choisissez des programmes et des cours qui vous intéressent, sans d'abord trop vous préoccuper de l'emploi que vous devrez trouver ou du salaire que vous ferez après avoir obtenu votre diplôme. Oui! Sans prendre en considération le marché du travail! Il s'agit de l'approche la plus intelligente, car, au bout du compte, vos cours doivent vous plaire et vous satisfaire, de la même façon que vous devez trouver votre travail satisfaisant après avoir obtenu votre diplôme. 
Par exemple, il ne sert à rien de choisir la comptabilité si c'est pour échouer vos cours parce que les bilans financiers et les rapports annuels ont sur vous un effet soporifique. Beaucoup d'étudiants choisissent des programmes et des cours en lien avec le commerce parce qu'ils (ou leurs parents) croient que c'est de cette façon qu'ils obtiendront un bon emploi. Le programme en commerce est difficile et rigide, et, après quatre années ou plus, certains diplômés préféreraient travailler dans n'importe quel autre domaine. Quel gaspillage de temps! Ne serait-il pas préférable d'obtenir un diplôme dans une matière qui vous plaît et pour laquelle vous voudrez toujours donner le meilleur de vous-même ? Vous pourrez toujours faire un Master of Business Administration (MBA - Maîtrise en administration des affaires) par la suite, à temps plein ou à temps partiel, si le commerce vous intéresse toujours. En somme, ça ne sert à rien de vous rendre malheureux; vous avez le choix. Et il devrait être le vôtre!

Comme vous le lirez plus loin dans ce livre, plusieurs chemins différents peuvent mener à votre emploi idéal. Celui que vous choisissez doit être celui qui vous attire le plus. Ne faites pas la grave erreur de croire qu'il faut absolument souffrir maintenant pour avoir du succès plus tard.

Choisir des cours qui vous intéressent constitue la meilleure façon de vous assurer que vous les réussirez, d'autant plus qu'obtenir de bons résultats (ce qui ne signifie pas nécessairement avoir des A partout) est essentiel à l'obtention d'un bon emploi. Le meilleur chemin vers l'emploi que vous voulez ne requiert donc pas que vous viviez des frustrations, que vous soyez malheureux, que vous échouiez des cours que vous détestez ou que vous changiez constamment de programme ou de majeure. Ce serait là une façon ridiculement coûteuse et douloureuse de se doter d'une éducation.

\section{Étudiants adultes, retour aux études et études à temps partiel}

Pour un tas de raisons différentes, y compris un épuisement professionnel ou burnout (presque tout le monde en souffre à un moment ou à un autre), il se peut que vous décidiez d'étudier à temps partiel ou de retourner aux études en tant qu'étudiant adulte. Ce qui est vraiment bien avec les collèges et les universités, c'est qu'ils sont toujours là pour vous! Vous pouvez donc choisir comment et où vous souhaitez apprendre.

Les études à temps plein ne conviennent pas à tout le monde et il existe des programmes à temps partiel dans la majorité des domaines 
d'études. Même si certains programmes ne permettent pas les études à temps partiel, ne vous laissez pas décourager; il y a beaucoup de façons créatives d'arriver à vos fins. Il y a de plus en plus de cours et de programmes en ligne, lesquels ont l'avantage d'être disponibles partout et en tout temps.

Étudier à temps partiel peut faire partie d'une bonne stratégie, que ce soit pour un été, pour une année ou pour toute la durée de votre programme. Les études à temps partiel mettent également en évidence une réalité indéniable du monde du travail : l'apprentissage ne s'arrête pas à un âge prédéterminé. Le temps où les études postsecondaires commençaient à la fin de l'adolescence pour se confiner à quelques années de collège ou d'université, et se terminer dans la jeune vingtaine, est en voie d'être révolu. Il ne faut pas s'accrocher à un modèle dépassé.

Ce n'est pas non plus un drame si vous ne pouvez pas entrer au collège ou à l'université dès votre diplôme d'études secondaires en poche. Le fait de ne pas étudier pendant quelques années pourrait même représenter un avantage quand vous demanderez l'admission à un programme d'études postsecondaires.

En fait, les professeurs (y compris les auteurs de ce livre) apprécient généralement les étudiants qui ont pris une pause des études. Il s'agit souvent d'étudiants hautement motivés, aux capacités d'écoute plus développées et qui savent déjà que le succès réside dans le fait de prendre des cours qui les intéressent.

Les étudiants qui font un retour aux études peuvent, par contre, avoir de la difficulté à voir le lien entre l'école et leur expérience de travail, ou l'inverse. Ils se concentrent parfois trop sur l'aspect pratico-pratique de leur apprentissage, c'est-à-dire qu'ils se préoccupent tellement de déterminer de quelle manière leur éducation correspond aux besoins du marché du travail qu'ils en oublient qu'apprendre est un plaisir. Au chapitre 11, nous traitons de quelques aspects en lien avec le retour aux études après avoir été sur le marché du travail pendant un certain temps.

\section{Quelles compétences applicables seront acquises pendant cette période?}

Pratiquement toutes les compétences que vous acquerrez et que vous utiliserez dans vos cours sont exactement celles dont vous aurez besoin pour avoir un emploi intéressant, stimulant et bien payé. Ça vous étonne? 
Faire des présentations orales, travailler en équipe, respecter les échéances, surmonter des épreuves, aborder des problèmes selon des perspectives différentes, résumer de l'information de façon concise, identifier des liens et des constantes, trouver et dépouiller des sources d'information, expliquer des événements passés et faire des projections pour le futur, écrire correctement et en faisant preuve d'une certaine profondeur analytique, et traiter avec des personnes en position d'autorité font très certainement partie de l'idée que vous vous faites de votre emploi idéal. Eh bien, ce sont ces compétences que vous pouvez, et que vous devez, acquérir au cours de vos études postsecondaires!

Prenez n'importe quelle occupation professionnelle ou poste de niveau supérieur dans les secteurs privé ou public et vous constaterez que les gens qui occupent ces emplois passent des heures à communiquer: ils convainquent les autres d'adopter de nouvelles idées ou propositions, ils obtiennent et interprètent de l'information et l'expliquent en personne ou par écrit. Ils consacrent également beaucoup de temps et d'efforts à comprendre les événements et les circonstances de façon critique et créative afin de régler des problèmes et apporter des améliorations: pourquoi avons-nous accusé des pertes ce trimestre? Comment pourrions-nous développer une stratégie plus forte et mieux adaptée ? Pourquoi certains de mes étudiants ontils échoué? Pourquoi y a-t-il eu des manquements à l'éthique dans notre compagnie? Y a-t-il un lien entre ce qui est arrivé aujourd'hui, ce qui s'est produit le mois dernier et ce qui pourrait arriver demain? Finalement, quiconque veut évoluer dans le monde professionnel doit être capable de bien travailler avec les autres, qu'ils soient superviseurs, collègues, subordonnés, clients ou autres.

Un des auteurs de ce livre a été directeur de placement étudiant pendant un an pour un programme de MBA dans une école prestigieuse, où les étudiants étaient recrutés par de grandes sociétés canadiennes et internationales. Ce que ces compagnies bien connues recherchent chez leurs employés potentiels, encore plus que des notes élevées, ce sont la capacité à résoudre des problèmes de façon créative, d'excellentes habiletés en communication, des aptitudes pour le travail d'équipe et une bonne capacité d'adaptation au changement.

Quand les recruteurs des sociétés de premier ordre évaluent les curriculums vitæ (CV) ou font passer des entrevues, ils essayent de détecter ces compétences universelles chez les candidats. Ils se débarrassent sans merci de façon experte de ceux qui n'ont qu'une bonne 
mémoire, qui ne pensent pas de façon créative et qui manquent d'entregent ou de flexibilité.

Même si les études postsecondaires constituent le meilleur investissement qu'une personne puisse faire, elles ne garantissent pas l'obtention d'un emploi qui vous permettra de vous épanouir. Vous devez utiliser vos années d'études pour développer et mettre en pratique les quatre principaux ensembles de compétences :

- communication;

- résolution de problèmes ;

- travail d'équipe ;

- adaptabilité.

La beauté de la chose, c'est qu'en le faisant, vous réussirez merveilleusement bien vos cours et vous trouverez le temps de profiter de tous les autres bénéfices qu'offre une éducation postsecondaire.

Les chapitres qui suivent vous donnent des trucs et des conseils sur la façon d'acquérir, dans vos cours, les compétences qui garantiront votre succès dans les études et au travail. Le chapitre 2 traite de manière approfondie des compétences générales que vous pouvez, et devez, développer au cours de vos études postsecondaires pour vous assurer de réussir dans le monde professionnel. Le chapitre 3 explique comment utiliser les examens, les dissertations et les rapports que vous produisez dans vos cours pour vous préparer à votre premier emploi et à votre carrière. Le chapitre 4 révèle comment acquérir les compétences critiques essentielles pour réussir au collège ou à l'université. Les chapitres 5 et 6 aident à développer vos aptitudes d'écoute et de lecture actives, et à renforcer vos habiletés en recherche. Les chapitres 7 et 8 abordent les aptitudes en résolution de problèmes nécessaires pour faire face aux diverses situations qui se présentent au cours des études, au bureau et dans la vie en général. Le chapitre 9 parle des rouages de la recherche d'emploi postcollégiale ou postuniversitaire. Le chapitre 10 traite des médias sociaux et de la façon d'en tirer profit. Enfin, le chapitre 11 termine le livre en jetant un coup d'œil au marché du travail et au-delà.

Une dernière chose avant de sauter dans le vif du sujet : les compétences qui mènent à la réussite, tant dans les études postsecondaires que sur le marché du travail, sont peut-être pratiquement identiques, mais elles doivent être adaptées à leur contexte propre. L'ensemble de compétences est peut-être le même, mais la période, les attentes et l'environnement dans lequel il est utilisé seront différents.

Personne n'a jamais affirmé que les études postsecondaires sont à l'image du monde réel. Par contre, le chemin qui mène des études 
postsecondaires au monde réel est simple et passionnant si vous savez ce que vous faites.

\section{Notes}

1. Statistique Canada, «Frais de scolarité pour les programmes menant à un grade, 2016-2017», Le Quotidien, 7 septembre 2016, http://www.statcan.gc.ca/dailyquotidien/160907/dq160907a-fra.pdf.

Statistique Canada, «Scolarité au Canada : niveau de scolarité, domaine d'études et lieu des études ", Recensement 2011, http://www12.statcan.gc.ca/nhs-enm/ 2011/as-sa/99-012-x/99-012-x2011001-fra.cfm.

2. Tel que rapporté par Statistique Canada dans son Enquête nationale auprès des diplômés, 2013, pour les diplômés de 2009-2010. Pour un résumé, consultez Josh Dehaas, « National Graduates Survey Shows Class of 2010 Did Well», Maclean's, 8 avril 2014, http://www.macleans.ca/work/jobs/national-graduate-survey-shows-classof-2009-10-did-well/. 


\section{Compétences pour réussir à l'école et au travail}

«Il n'y a qu'une chose plus douloureuse que d'apprendre de l'expérience et c'est de ne pas apprendre de l'expérience.» - Archibald Macleish

\footnotetext{
Omme nous l'avons mentionné dans le chapitre précédent, tous les professionnels du monde du travail doivent savoir communiquer, résoudre des problèmes, travailler en équipe et s'adapter au changement. Si vous ne maîtrisez pas au moins un de ces ensembles de compétences, dites tout de suite adieu à vos perspectives de carrière. Par contre, savoir communiquer constitue peut-être le plus important des quatre ensembles. Après tout, une avocate, un travailleur social, une enseignante, un directeur d'entreprise, une journaliste ou tout autre professionnel du marché du travail pourrait-il réussir sans posséder des habiletés en communication hors pair?

Dans ce chapitre, nous expliquons comment l'un des aspects les plus négligés et malaimés des études postsecondaires vous sera des plus utiles dans vos activités futures. Notre expérience, ainsi que celle de plusieurs récents diplômés, parle d'elle-même : faire des présentations, travailler en groupe, faire preuve d'initiative, prendre des notes et organiser vos tâches ne vous faciliteront pas seulement la vie à l'école, mais aussi dans votre emploi après les études. Commençons avec les présentations orales.
} 


\section{Les présentations orales}

Pour plusieurs étudiants, faire une présentation dans un cours ou un tutoriel est un des aspects les plus pénibles des études postsecondaires. Les symptômes qui accompagnent la grippe des présentations incluent le plus souvent: mains moites, papillons dans l'estomac et paralysie totale. Les présentations sont stressantes, les préparer prend du temps et on ne sait jamais à quoi s'attendre. Malgré tout, elles représentent une des activités des études postsecondaires les plus directement liées au marché du travail.

Nous utilisons ici une définition englobante des présentations orales. Du côté formel du spectre des présentations, vous devrez probablement parler devant la classe sur un sujet choisi par votre professeur ou par vous. Mais ce type de présentation ne diffère qu'en intensité des interactions plus informelles en salle de classe. Chacune d'entre elles est toutefois importante pour réussir à l'école.

Il en est de même dans le monde du travail. Sans de bonnes habiletés en présentation, vous aurez de la difficulté à décrocher un emploi. Après tout, la première présentation orale que vous ferez pour votre employeur sera votre entrevue! Et puisque les emplois d'aujourd'hui deviennent rarement des emplois pour la vie, vous devrez subir cette épreuve plusieurs fois si vous voulez progresser dans votre carrière ou même seulement garder votre emploi.

Il y a donc de fortes chances que les présentations jouent un rôle majeur dans votre vie professionnelle. Qu'elles soient formelles et prononcées devant un grand auditoire ou données informellement lors d'un déjeuner ou un dîner d'affaires, elles constituent le moyen privilégié de partager de l'information dans le monde professionnel. Si vous devenez directeur ou propriétaire d'une entreprise, une bonne partie de vos journées sera consacrée à préparer et à faire des présentations de toutes sortes, ainsi qu'à y réagir. Et à moins que votre employeur ne vous enferme au troisième sous-sol et ne vous y oublie, vous ferez plusieurs présentations dans le cadre de vos fonctions. Vous devrez assurément expliquer quelque chose à un moment donné, communiquer vos idées aux autres et encourager les gens avec qui vous interagirez. Clients, investisseurs, collègues, superviseurs et peut-être le grand public composeront votre auditoire. Même si vous ne faites pas beaucoup de présentations au cours de votre premier emploi, vous devrez probablement en préparer pour votre superviseur. Dans tous les cas, vous ne ferez pas bonne impression auprès 
de votre patron et de vos collègues si vos habiletés en communication sont nulles.

À cause de la place importante qu'occupent les présentations dans le monde du travail, plusieurs carrières prometteuses ont été sabotées par l'incapacité à bien communiquer. Pourquoi donc ne pas apprendre à bien le faire maintenant, alors que les enjeux ne sont pas aussi élevés? Profitez de vos années d'études postsecondaires pour vous exercer. Si vous devez faire une présentation formelle pour un cours, essayez de la planifier tôt pendant la session pour qu'il y ait moins de risques de conflit avec d'autres tests et travaux. Apprenez également à utiliser PowerPoint ou un autre logiciel de présentation. Votre employeur, vos collègues et vos clients s'attendront à ce que vous sachiez le faire de toute façon et que vous soyez en mesure de manipuler toute autre technologie du même genre. C'est dans de telles situations que vous serez récompensé pour le temps et les efforts investis dans vos présentations scolaires.

Si vos cours n'exigent pas que vous fassiez de présentations formelles, ils offrent tout de même beaucoup d'occasions de développer vos compétences oratoires. Poser des questions au professeur ou à l'assistant d'enseignement est une présentation orale miniature. Parler à un instructeur pendant ses heures de bureau ou dans toute autre situation est aussi une forme de présentation, car vous devez vous y préparer pour être efficace.

Il est stressant de faire une présentation en classe, mais moins si vous suivez les étapes ci-dessous. Vous allez peut-être même finir par aimer l'expérience! N'oubliez pas le conseil principal (et le meilleur!) de ce livre. Il est beaucoup plus facile de faire une présentation si le sujet vous intéresse. Après tout, pourquoi parler de quelque chose qui vous ennuie à mourir? Par conséquent, la clé est de suivre des cours (et, en fin de compte, d'avoir une carrière) qui vous satisfont. Même si vous êtes pris avec un mauvais sujet de présentation - que ce soit parce que les autres étudiants ont choisi les meilleurs ou parce que le professeur vous l'a assigné -, ne vous contentez pas de la médiocrité. Seuls ceux qui manquent de créativité n'arrivent pas à trouver quelque chose d'intéressant dans un sujet peu attrayant. Embaucheriez-vous quelqu'un comme ça? Il est inévitable qu'à un moment ou à un autre de votre carrière, vous devrez parler d'un sujet qui ne vous intéresse pas.

Vous exercer à l'école peut donc vous donner le savoir-faire nécessaire pour bien y arriver. Ce qui nous mène au secret par 
excellence du succès de tout présentateur: l'enthousiasme! Il n'y a, en effet, rien de plus contagieux, de plus efficace et de plus convaincant que l'enthousiasme. Les meilleurs enseignants et les meilleurs présentateurs sont ceux qui parviennent à transmettre leur enthousiasme personnel et leur passion. Quand on combine l'enthousiasme et la passion au savoir et à un peu de pratique, on obtient un résultat final génial. Consacrer autant de travail à une courte présentation peut vous sembler beaucoup de temps et d'efforts pour si peu. Mais ça ne l'est pas vraiment, en partie parce que plus vous vous préparerez et vous exercerez, plus facile sera la prochaine présentation. Vous trouverez peut-être que vous manquez de temps pour vous préparer ; c'est la vie! Une fois que vous aurez un emploi à temps plein, avec des responsabilités, vous aurez encore moins de temps pour vous préparer. Votre patron, vos clients et vos collègues s'attendront à ce que vous soyez efficace dès le départ. Quand il vous demandera de parler d'un sujet précis, votre patron pourrait ne vous donner qu'un court préavis, voire pas de préavis du tout. Pourquoi ne pas profiter de vos années d'études postsecondaires pour devenir un bon communicateur? Vous pensez que certaines personnes sont meilleures que d'autres pour communiquer oralement? À vrai dire, n'importe qui peut s'améliorer significativement en communication orale. Un des auteurs de ce livre souffre d'un handicap que la plupart d'entre vous n’ont pas: il bégaie. Et quand il était au secondaire et à l'université, il n'a jamais levé la main ou répondu à une seule question. Il a néanmoins réussi à devenir professeur et conférencier ainsi que communicateur hautement efficace.

Quand on excelle à quelque chose de difficile, on éprouve beaucoup de satisfaction, voire de plaisir, à le faire. Faire des présentations est de toute évidence difficile, et donc en faire une qui est efficace peut être incroyablement satisfaisant. Vous n'avez qu'à regarder les visages de vos collègues de classe après une présentation réussie pour vous en convaincre.

Abordez les présentations comme une chance de développer une compétence utile, de prouver votre capacité à simplifier la matière et à impressionner vos professeurs et vos collègues (sans oublier la personne la plus importante: vous-même). Voici quelques trucs faciles pour les présentations formelles, que vous pouvez adapter à votre situation:

- Faites preuve d'initiative. Voyez toujours une présentation comme une chance d'impressionner tout le monde, non 
seulement en leur montrant à quel point vous avez travaillé fort, mais aussi par votre intelligence et votre originalité ;

- Faites une répétition générale. C'est en forgeant qu'on devient forgeron! Exercez-vous devant le miroir, vos frères et sœurs, vos amis, peu importe. Filmez-vous ou enregistrezvous. Il s'agit là d'un exercice utile pour toute présentation, qu'elle dure plusieurs minutes ou qu'elle soit une simple question lors du cours du lendemain ;

- L'apparence compte pour beaucoup. Habillez-vous bien pour montrer à tout le monde que vous prenez votre présentation au sérieux et que vous respectez votre auditoire ;

- Arrivez tôt. Vérifiez votre équipement. Relisez vos notes. Décidez de la façon dont vous accueillerez les gens ;

- Accueillez les gens chaleureusement. Faites de votre auditoire votre allié dès le début. Présentez-vous, en plus de votre sujet. Rappelez-vous que ceux qui vous écoutent veulent que vous réussissiez;

- Souriez beaucoup, mais ne faites pas le fanfaron. Une mauvaise blague pourrait tout gâcher ;

- Ayez l'air en confiance et en contrôle, même si vous avez des papillons dans l'estomac;

- Parlez directement à votre auditoire. Ne lisez jamais vos notes, si possible, et ne les apprenez pas par cœur. Vous risqueriez de sonner comme un robot. Référez-vous à vos notes seulement si vous avez un blanc de mémoire ;

- Demeurez vous-même, mais faites attention aux manies. Tout le monde a des tics nerveux. Détectez-les, éliminez-les ou transformez-les en vous exerçant devant un miroir ou en vous filmant;

- Variez les activités. Rappelez-vous que l'auditoire a une capacité d'attention réduite ;

- Donnez à l'auditoire un résumé clair qui pourrait lui être utile comme guide d'étude pour un examen. Les autres étudiants apprécieront d'autant plus votre présentation que vous leur épargnerez un peu de travail ;

- Assurez-vous de toujours avoir au moins une surprise en banque. Si vous fournissez une valeur ajoutée à laquelle personne ne s'attend, tout le monde se souviendra de votre présentation. Si c'est pertinent, apportez des bonbons ou d'autres friandises que vous distribuerez. Soyez créatif ; 
- Finissez de façon percutante et ne soyez pas trop modeste. La fausse modestie est pour les perdants. Les gagnants s'efforcent toujours de terminer en force ;

- Personne n'aime la critique. Essayez de la voir de façon constructive, sans être sur la défensive si on vous pose des questions après la présentation. Répondez de façon succincte et positive, avec une formule du genre: "C'est un point de vue intéressant, merci. »

Vous croyez peut-être que les présentations orales ne méritent pas qu'on leur accorde autant d'importance? Pensez un peu aux raisons qu'ont les professeurs et les employeurs de les préférer à d'autres façons de transmettre de l'information ou des idées :

- Les présentations constituent la façon la plus personnalisée de transmettre la matière. Vous n'avez qu'à penser à leur pouvoir de persuasion ;

- La moitié des communications interpersonnelles se font sous la forme d'expressions faciales et de langage corporel ;

- Les gens apprennent mieux quand ils voient et entendent en même temps. Une bonne présentation permet à l'auditoire de voir et d'entendre le présentateur. L'utilisation appropriée de diapos ou d'autre matériel aide l'auditoire à assimiler l'information et à s'en souvenir ;

- Un texte écrit rend difficilement les styles rhétoriques et les outils utilisés par un bon communicateur. Celui-ci peut produire un effet par une pause, s'ajuster en fonction des réactions, changer de ton et d'accent, inviter l'auditoire à poser des questions et y répondre;

- Une présentation bien préparée et bien rendue exprime un engagement réel envers l'auditoire. En faisant preuve d'autant de respect pour ceux qui les écoutent, les présentateurs gagnent davantage de respect pour leur sujet qu'il n'est possible d'en obtenir sous toute autre forme.

Les mauvais présentateurs, ou ceux qui sont inexpérimentés, ont tendance à faire les six mêmes erreurs :

- Ne pas parler assez fort. La meilleure présentation du monde est inefficace si personne ne peut l'entendre. Exercez-vous à parler à plusieurs endroits afin de vérifier que tout le monde dans la pièce peut vous entendre. Plusieurs présentateurs ne parlent pas assez fort et ont tendance à marmonner, surtout quand ils se penchent pour lire leurs notes. Ceux dont la 
présentation est inaudible ne font qu'indiquer qu'ils n'ont pas envie d'être là, qu'ils ne savent pas trop de quoi ils parlent et qu'au fond, ils s'en foutent;

- Ne pas regarder l'auditoire dans les yeux. Les présentations permettent d'interagir et d'établir un rapport privilégié avec l'auditoire, ce qui est impossible à faire si l'orateur ne regarde pas l'auditoire dans les yeux. Éviter de regarder l'auditoire trahit de l'incertitude et un manque d'engagement à l'égard du sujet ou de la présentation elle-même ;

- Donner trop d'informations. Il s'agit là de la pire erreur des orateurs inexpérimentés et qui manquent d'assurance ; ils tentent souvent d'en faire trop. En règle générale, un auditoire ne peut pas absorber plus que trois grandes idées, illustrées par deux ou trois exemples chacune. Si on lui en donne plus, il ne porte plus attention. Déterminez ce qui doit absolument être transmis et laissez le reste de côté. De toute façon, aucune présentation ne pourra jamais communiquer tout ce que vous savez sur votre sujet, ni même la majorité des connaissances que vous avez sur celui-ci ;

- Prendre plus que le temps alloué. Que pensez-vous des professeurs qui continuent de parler après la fin du cours? Il n'y a pas meilleure façon de saboter une bonne présentation que de dépasser le temps alloué. Déborder donne l'impression à l'auditoire que votre temps est plus important que le sien, ce qui s'oppose à l'atmosphère de respect mutuel que vous avez essayé de créer. Si vous croyez que vous ne terminerez pas à temps, laissez simplement du matériel de côté. Faitesnous confiance, personne ne s'en rendra compte. Pour éviter de déborder, faites une présentation qui est plus courte que le temps alloué. Vous vous assurerez ainsi non seulement de terminer à temps, mais aussi d'avoir assez de temps pour les questions à la fin ;

- Distribuer du matériel. Rendez le matériel disponible avant ou après votre présentation. Distribuez-le pendant que vous parlez seulement si vous comptez le parcourir dans son entièreté avec votre auditoire. Autrement, ce dernier ne vous portera plus attention. Certains commenceront à lire le matériel tout de suite et vous devrez redoubler d'ardeur pour regagner leur attention. Il est beaucoup plus intelligent de ne pas la perdre du tout ; 
- Utiliser des appuis audiovisuels de façon inappropriée. Ne soyez pas séduit par la technologie! Une présentation réussie dépend rarement d'effets spéciaux audiovisuels, lesquels peuvent représenter une distraction de taille s'ils sont mal utilisés ou s'ils ne fonctionnent pas correctement. Si vous projetez des diapos à l'écran, ne vous contentez pas de les lire ; utilisez-les plutôt pour vous guider ou pour mettre en relief vos thèmes principaux. Assurez-vous que les appuis visuels sont :

o bien utilisés et pertinents au contexte ;

o utilisés pour appuyer vos arguments et non pas pour en distraire ;

o simples et faciles à voir ou à entendre.

Pour plusieurs, les questions de l'auditoire, en particulier celles du professeur (ou du patron), représentent la partie la plus stressante d'une présentation. Rappelez-vous que c'est vous qui menez. Vous avez fait les recherches nécessaires et vous vous êtes bien préparé. Vous êtes l'expert en la matière. En effet, pour quiconque a lu ou compris moins que vous sur le sujet (y compris votre professeur), c'est vous l'expert! C'est vous qui contrôlez la dynamique de la présentation et qui structurez le cadre des questions et des réponses.

Établissez donc d'emblée les règles du jeu pour profiter de vos forces. Si vous préférez garder les questions pour la fin, mentionnez-le au début. D'un autre côté, si vous voulez favoriser les interactions, vous pouvez demander à l'auditoire de poser des questions pendant votre présentation.

Encourager l'auditoire à poser des questions est une bonne façon de l'inviter à établir un lien avec vous. C'est cette invitation, plutôt que les questions, qui permet de créer ce lien.

L'invitation à poser des questions doit être sincère. Ne vous contentez pas de dire simplement : « Avez-vous des questions ? », puis de vous rasseoir. L'auditoire doit avoir le temps de réagir pour interagir avec vous. Donnez-lui le temps de le faire. Dites quelque chose comme: "J'espère que vous avez quelques questions à me poser» ou «Est-ce qu'il y a quelque chose que vous aimeriez que je clarifie ou qui mérite d'être discuté davantage? » Regardez les gens dans les yeux. Souriez. Attendez. Vingt secondes peuvent ressembler à 20 minutes quand on attend que quelqu'un parle, mais les gens ont besoin de temps pour passer du mode écoute au mode question. Attendez que ça se produise. 
Chaque fois qu'on vous pose une question, répétez-la pour l'auditoire. Cela prouvera que vous la prenez au sérieux (gratifiant du même coup la personne qui l'a posée) et encouragera le reste de l'auditoire à participer.

Reformulez positivement les questions négatives, qu'on appelle aussi parfois «interjections » ou «interventions ». Elles sont posées habituellement par quelqu'un qui essaie de faire étalage de son intelligence au détriment de la vôtre. N'essayez pas de faire taire la personne puisque cela viendrait à l'encontre de votre accessibilité en tant que présentateur. Mettez simplement en évidence ce qu'il y a de positif.

Ne prétendez jamais savoir si vous ne savez pas. Ne perdez pas non plus votre sang-froid. Si vous ne connaissez pas la réponse à une question, dites-le. Mais ne vous arrêtez pas là : ajoutez qu'il vous fera plaisir de trouver l'information pour quiconque est intéressé. Si vous avez confiance que vous serez capable de reprendre le contrôle de la discussion, vous pouvez même poser la question à l'auditoire, au cas où quelqu'un aurait la réponse. N'essayez cela que si vous savez comment rediriger l'attention vers vous par la suite. Mettez poliment un terme à toute question ou discussion qui ne mène nulle part. N'oubliez pas : c'est vous qui menez la discussion. L'auditoire s'attend à ce que vous empêchiez gentiment les gens de s'éterniser et que vous redirigiez les sujets sans issue. Rappelez à tous pourquoi vous intervenez à l'aide d'une phrase comme: «Je vois que d'autres personnes attendent de parler. Pouvez-vous terminer votre question? ? ou «Je suis sûr qu'il y a beaucoup d'autres problèmes à soulever ; nous allons donc passer à une autre personne.»

Si quelqu'un pose une mauvaise question, convertissez-la en bonne question, profitant de l'occasion pour exposer votre talent et séduire votre auditoire. Les présentateurs d'expérience réussissent à trouver du bon dans une mauvaise question et à en tirer parti. Celui qui a posé la question ne remettra que très rarement en doute l'interprétation que vous en ferez, puisque la reformulation que vous en proposez le mettra à son avantage. Ceux qui sont perspicaces apprécieront le fait que vous avez évité une situation embarrassante sans faire offense à la dignité de quiconque. Tout le monde en sortira gagnant.

Le plus important, c'est de ne pas être sur la défensive et d'essayer de justifier votre position quand vous répondez aux questions. Il n'est pas facile d'entendre: "vous avez oublié ceci» ou «je ne suis pas d'accord », voire "vous avez tort». Votre réaction naturelle sera de vous lancer dans une longue explication ou de répéter certaines 
parties de votre exposé. N'en faites rien. Répondez plutôt brièvement à toutes les questions et n'hésitez pas à dire : «C'est un excellent argument que vous apportez. J'en prends note.»

Remerciez toujours les gens d'avoir posé des questions et ditesleur qu'ils ont été un bon auditoire. Si vous le faites, ils n'auront d'autre choix que de conclure que vous êtes un présentateur efficace!

Nous espérons que vous commencez maintenant à voir les présentations orales sous un autre jour, non plus comme des tâches pénibles, mais bien comme des activités intéressantes de grande valeur qui peuvent même être amusantes! Passons maintenant à une autre activité souvent détestée par les étudiants, mais qui est presque aussi essentielle sur le marché du travail.

\section{Les travaux de groupe}

Au cours de vos études secondaires, vous avez probablement dû faire quelques travaux de groupe. Dirigés par un bon enseignant, ils peuvent être passionnants. Mais il arrive souvent que certains membres du groupe travaillent plus fort que d'autres, même si tout le monde reçoit le crédit pour le travail. Une fois au collège ou à l'université, plusieurs étudiants croient donc que les projets de groupe ne reconnaissent pas l'effort individuel et les perçoivent comme une perte de leur précieux temps.

Ne laissez pas les expériences négatives du passé influencer votre appréciation de la valeur des projets de groupe. Dans le monde du travail, rares sont ceux qui travaillent isolément. La collaboration efficace se trouve à la base du succès des compagnies et des organisations. Et, contrairement à ce qui se passe au secondaire, si vous ne contribuez pas au succès du groupe, votre réputation en prendra un coup et vous ne ferez pas long feu au sein de la compagnie. De nos jours, la communauté d'affaires et le marché de l'emploi sont de grands amateurs de la collaboration. Il n'est donc pas étonnant que les présentations dans les écoles de commerce et dans beaucoup de programmes professionnels postsecondaires soient faites en équipe.

Les présentations de groupe ont ceci de fascinant que le produit final est toujours bien meilleur que la somme de ce que chaque individu aurait pu produire seul. La raison en est simple: chacun apporte sa propre expertise.

Malgré ces avantages, le travail d'équipe comporte sans contredit des désavantages. Il y a presque toujours des différences d'opinions 
quant à la façon de procéder, se mettre d'accord prend du temps et tout le monde ne travaille pas aussi fort.

Vous devez toutefois apprendre à composer avec ces frustrations. Dans votre futur emploi, vous travaillerez principalement en groupe. Rédiger seul un document (comme une proposition) et le soumettre à une seule autre personne pour révision est chose rare sur le marché du travail. La plupart des activités se font en groupe. En fait, être capable de bien travailler en équipe vous permettra d'obtenir des emplois, alors que l'incapacité à le faire vous les fera perdre. Il en ira de même pour vos promotions.

Dans le monde professionnel, le travail d'équipe est crucial puisque presque toutes les activités contribuent à des projets complexes qui dépassent les compétences d'une seule personne. Peu importe l'emploi que vous aurez, vous devrez être en mesure de travailler avec une ou plusieurs équipes. Même si vous travaillez à votre compte, vous devrez collaborer avec vos clients, vos fournisseurs et plusieurs autres intervenants.

Utilisez vos années d'études postsecondaires pour parfaire votre habileté à collaborer avec les autres. Essayez de vous joindre à différents types de groupes: hommes et femmes, étudiants plus vieux et plus jeunes, étudiants étrangers ou qui viennent de milieux différents du vôtre. Jouez des rôles variés au sein de ces groupes : prenez parfois le contrôle et laissez parfois les autres le prendre.

Observez comment les membres du groupe interagissent entre eux. Regardez comment ils agissent et réagissent. Pensez aux façons grâce auxquelles l'équipe pourrait fonctionner différemment. Réfléchissez à la manière dont le sexe, l'origine ethnique et d'autres facteurs en influencent le fonctionnement. Portez attention à la façon dont le produit final bénéficie de cette variété d'expériences et de points de vue. Les présentations de groupe et les projets collaboratifs présentent des difficultés particulières. Voici des conseils simples, applicables à n'importe quelle situation de groupe :

- Faites toujours une séance de remue-méninges avant de décider de la stratégie à adopter ;

- Ne laissez pas une seule personne décider du plan. Tous doivent sentir que le projet leur appartient ;

- Assurez-vous que tous ont un rôle précis à jouer pour mener à bien le projet. Assurez-vous également que tous comprennent qu'avoir une responsabilité particulière ne les soustrait pas à celle de l'ensemble du projet ; 
- Si la pression des pairs n'est pas suffisante, soyez prêt à éjecter les membres qui traînent de la patte. Cela peut s'avérer difficile si vous êtes étudiant, mais le groupe, en tant qu'entité, peut demander de l'aide à l'instructeur (et plus tard à votre patron!) pour qu'il s'occupe de ceux qui ne font pas leur part ;

- Agissez en équipe pendant la présentation ou le projet. $\mathrm{Si}$ vous n'avez pas de rôle particulier à jouer, appuyez vos collègues ou manifestez votre solidarité aux clients ou aux auditoires. Soyez toujours prêt à fournir de l'aide à vos collègues s'ils rencontrent des difficultés ;

- N'essayez pas d'en mettre plein la vue. Certaines personnes ont tendance à prendre les commandes et à dominer le groupe, mais cette attitude entraîne des répercussions négatives sur la présentation ou sur l'ensemble du projet ;

- Terminez les choses en beauté. Célébrez la fin du projet avec vos collègues. Bien que brièvement, vous avez été une équipe efficace et vous voudrez en être reconnaissants et respectueux les uns envers les autres.

Comme dans le cas des présentations orales, nous espérons que vous percevez maintenant la valeur et l'intérêt des travaux de groupe. Sautez sur toutes les occasions de développer les compétences qui leur sont liées. L'efficacité de la collaboration est une notion que tout employeur progressif comprend. Ainsi, si vous voulez travailler pour une compagnie ou une organisation avant-gardiste, soyez prêt à abandonner tout espoir de tout faire seul. La vraie magie du travail de groupe est qu'il vous permet d'accomplir, en partenariat avec d'autres, beaucoup plus que ce que vous pourriez faire seul. Le résultat vaut la peine de surmonter quelques frustrations en cours de route et le temps qu'on y passe n'est pas perdu.

\section{Faire preuve d'initiative en tant que professionnel}

Les gens qui ont de l'initiative, qui ont l'âme d'un entrepreneur, sont hautement prisés dans les organisations publiques et privées. Le problème, selon les professeurs de collège et d'université, et les employeurs, c'est que trop d'étudiants et de diplômés sont passifs et s'attendent à ce qu'on leur serve tout sur un plateau d'argent. Si cette situation s'applique à vous, vous n'êtes pas prêt à affronter la vraie vie.

Jusqu'à un certain point, les cours que vous suivez pendant vos études postsecondaires ne sont pas très différents du travail que 
vous ferez probablement après avoir terminé vos études. Comme le font vos professeurs et les assistants à l'enseignement, votre patron ou votre client vous donnera des instructions vagues et s'attendra à ce que vous les compreniez. Si vous cherchez un emploi comportant des instructions claires et détaillées, il ne s'agira probablement pas de l'emploi de vos rêves et certainement pas d'un poste destiné à un professionnel.

Les « Mc-emplois » se caractérisent par le fait qu'ils ne demandent pas aux employés de penser par eux-mêmes ou d'avoir de l'initiative. C'est pourquoi ils sont aussi mal rémunérés. Si vous voulez un bon emploi, vous devez être capable de travailler sans avoir besoin que quelqu'un vous prenne par la main pour vous guider. Vous devrez faire preuve d'initiative.

À l'école, agissez avec le professionnalisme que vous prévoyez avoir sur le marché du travail. Remettez vos travaux à temps et ne soyez pas en retard aux cours. Lisez attentivement le plan de cours. Faites les lectures requises avant le cours pour pouvoir bénéficier de l'interprétation du professeur. Si vous ne prenez pas vos cours au sérieux, les risques sont élevés que vous agissiez de la même façon dans votre emploi futur.

C'est là la véritable raison qui explique pourquoi les employeurs veulent connaître vos résultats scolaires. Ceux qui obtiennent de bonnes notes sont consciencieux, dévoués et prennent des initiatives pour réaliser les tâches qu'impliquent les études postsecondaires. Un A suppose une bonne préparation et une volonté d'aller au-delà des attentes.

Essayez d'abord de comprendre les choses par vous-même au lieu de consulter votre assistant à l'enseignement ou votre professeur dès que vous avez une question. Votre patron, vos clients et vos collègues n'apprécieront certainement pas que vous les dérangiez trop souvent avec vos questions relativement sans importance ou votre besoin constant d'être rassuré.

Demander de repousser la date limite de remise d'un travail le jour même où vous devez le remettre peut être acceptable dans certains cours, mais cette approche entravera très certainement votre avancement et votre carrière, une fois vos études postsecondaires terminées. Vous effondrer devant votre professeur la veille de l'examen final à cause du stress, du manque de sommeil ou d'une mauvaise alimentation peut, peut-être, inspirer de la sympathie quand vous êtes au collège ou à l'université. Un effondrement semblable 
devant votre patron ou votre client vous coûtera votre promotion, voire votre emploi.

Petit conseil : évitez de faire une fixation sur vos notes quand vous parlez à un professeur. N'hésitez pas à demander comment faire pour mieux comprendre le matériel ou pour améliorer vos résultats. Après tout, les notes ne sont que le reflet de votre compréhension et de vos connaissances.

De la même façon, votre patron ou vos clients réagiront mal si vous leur parlez de salaire ou si vous leur demandez s'ils sont satisfaits de votre travail. Ils seront toutefois toujours prêts à discuter du travail que vous avez fait.

Vous devrez parfois demander quelque chose à un professeur ou à un assistant à l'enseignement. Encore une fois, abordez la situation en professionnel : préparez votre dossier ou vos questions, exercez-vous et essayez d'analyser la situation. Les professeurs sont des professionnels et les assistants à l'enseignement, des professionnels en formation. Leur emploi du temps est chargé et l'enseignement n'est qu'une partie de leur travail. Ils ont donc parfaitement le droit d'exiger que vous ne leur fassiez pas perdre leur temps.

Vous croyez peut-être que la différence entre les études postsecondaires et le marché du travail est si grande qu'il importe peu que vous remettiez vos travaux en retard, rédigiez vos dissertations la veille de la date limite, fassiez du bourrage de crâne, séchiez vos cours, fassiez le minimum et soyez désorganisé, car dès que vous recevrez votre diplôme, vous deviendrez une personne complètement différente. Ce genre de métamorphose n'arrive que très rarement. Généralement, la façon dont vous agissez pendant vos études donne une bonne indication de celle dont vous agirez après avoir obtenu votre diplôme. Les employeurs potentiels sont certainement conscients de cela. Après tout, ce n'est pas la première fois qu'ils embauchent des diplômés.

Évidemment, la salle de classe n'est pas du tout comme le bureau. Vous avez beaucoup plus de liberté en classe pour vous exprimer et approfondir vos intérêts personnels que vous en aurez au travail. Si vous choisissez de manquer des cours ou de ne pas participer aux discussions en classe, vous n'apprendrez pas grand-chose (après tout, c'est vous qui payez pour votre éducation), mais ce sera probablement la seule répercussion. Quoi qu'il en soit, le degré de professionnalisme dont vous faites preuve au cours de vos études postsecondaires est le meilleur indicateur de ce qu'il sera après l'obtention de votre diplôme. 
On peut acquérir bien d'autres habiletés à l'école. Il est vrai que plusieurs d'entre elles peuvent s'acquérir d'une autre manière, comme en voyageant ou en travaillant à temps partiel. Mais l'avantage des études postsecondaires à cet égard, c'est qu'elles ont été créées pour faciliter l'apprentissage et renforcer les habiletés et les compétences. Par exemple, la diversité culturelle est importante pour les collèges et les universités, car elle permet d'élargir les horizons de l'esprit et de puiser à même des connaissances qui proviennent de sources très variées. Les collèges et les universités ne sont pas les seuls endroits où on peut en apprendre sur différentes cultures, mais la plupart de vos professeurs voudront plus volontiers vous aider à explorer les différences culturelles. De plus, il est beaucoup plus facile d'en apprendre sur les autres quand ils sont vos pairs et qu'ils font partie du même laboratoire que vous, conçu pour favoriser l'apprentissage.

L'information sur les autres cultures est précieuse dans n'importe quelle profession qui souhaite tirer son épingle du jeu dans l'économie mondiale actuelle. Saviez-vous que, parmi les Asiatiques, seuls les Coréens utilisent le nom de famille «Park» et que c'est le nom de $25 \%$ des Coréens? Eh bien! Maintenant, quand vous aurez votre superemploi et que vous rencontrerez un client asiatique qui s'appelle «Park», vous aurez une longueur d'avance.

Il peut être très utile d'avoir une connaissance approfondie des autres cultures. Les organisations nord-américaines ont en effet beaucoup à apprendre de leurs homologues asiatiques. Les sociétés japonaises et coréennes réussissent bien, en partie parce qu'elles mettent autant l'accent sur la dynamique de groupe que sur les réalisations individuelles, et parce qu'elles adoptent des perspectives à long terme.

À l'université et au collège, on vous encourage à explorer systématiquement les connexions comme celles établies entre la culture et le commerce. Ce serait beaucoup plus difficile, et imprudent, d'apprendre ce genre de chose au travail. Vous ne voudrez probablement pas demander à un nouveau client d'expliquer un aspect particulier de sa culture ou de sa famille.

En fait, puisqu'il est pratiquement assuré que vous ferez affaire avec des clients, des collègues et des patrons qui proviennent de cultures différentes, pourquoi ne pas prendre une longueur d'avance pendant vos études postsecondaires? Certains de vos camarades de classe sont-ils d'une autre culture ou proviennent-ils d'une autre région du globe? Pourquoi ne pas apprendre d'eux? Si vous intégrez systématiquement vos apprentissages formels et informels, vous 
multiplierez de manière exponentielle les bénéfices de vos études postsecondaires.

Comment être systématique à cet égard ? Mémorisez quelques phrases dans une langue étrangère. Apprenez comment dire bonjour correctement (incliner la tête, serrer la main, faire la bise sur une joue ou sur les deux). Informez-vous sur les gens qui proviennent d'horizons différents. Explorez un peu plus en profondeur les autres cultures (et perspectives).

Ne tenez jamais pour acquis que votre communauté ethnique ou votre groupe socioéconomique détient toutes les réponses. Une connaissance plus vaste et plus approfondie fera de vous un meilleur professionnel, peu importe le domaine dans lequel vous travaillerez, du commerce à la science, en passant par le travail social.

Un fait important à considérer est que quelques-uns de vos camarades de classe pourraient devenir vos clients ou vos collègues. Les entrepreneurs et les cadres supérieurs nomment souvent leurs colocs de collège ou d'université dans des postes élevés. Les gestionnaires à la recherche de nouveaux employés consultent souvent leurs connaissances. Le réseautage informel et le bouche-à-oreille ont beaucoup plus de poids que les demandes d'emploi formelles. Les connaissances que vous vous faites ne sont peut-être pas aussi importantes que ce que vous apprenez, mais il reste que savoir comment construire votre réseau de contacts est essentiel (consultez le chapitre 9 pour plus de détails sur le réseautage).

Bien qu'elles ne soient pas enseignées en salle de classe, certaines compétences générales vont de pair avec les études postsecondaires. Par exemple, on y encourage et appuie les étudiants qui souhaitent acquérir de nouvelles habiletés, comme écrire, faire de la recherche et maîtriser les technologies de l'information. Les minicours et autres ateliers semblables offerts à l'extérieur du campus vous coûteront les yeux de la tête.

La technologie change constamment, mais ne jouez pas à l'autruche. Essayez de vous tenir au courant des plus récents développements dans ce domaine. L'utilisation d'un logiciel de traitement de texte de base ne constitue plus un avantage pour l'obtention d'un emploi. Beaucoup d'employeurs veulent du personnel qui peut, à tout le moins, utiliser des tableurs Excel, des logiciels de graphisme et de visualisation, et des outils en ligne. Si vos cours n'incluent pas ce genre d'apprentissage, profitez des ateliers gratuits offerts sur le campus, habituellement par l'entremise de la bibliothèque ou du centre 
d'aide à la rédaction. Ils vous aideront à développer vos compétences dans ce domaine.

Finalement, un établissement postsecondaire, en particulier s'il est de taille importante, est un endroit où vous pouvez trouver du travail et des occasions d'accumuler de l'expérience. Vous y trouverez des postes au sein du régime travail-études et d'autres offerts par des professeurs pour les aider à mener des recherches, faire des entrevues ou animer des laboratoires informatiques. De plus, il y a des dizaines de clubs, d'équipes sportives, d'associations, de stations de radio et de troupes de théâtre qui sont souvent à la recherche de personnel.

Au moment d'embaucher des diplômés récents, plusieurs sociétés scrutent les $\mathrm{CV}$ pour savoir si les candidats se sont impliqués dans les clubs ou dans les équipes sportives. Pourquoi ? Parce que la plupart des employeurs potentiels sont impressionnés par quiconque peut combiner études et activités parascolaires; ces candidats font preuve d'équilibre, d'engagement, de volonté de travailler dur et de le faire en équipe.

N'oubliez pas le plus évident : vous joindre à un groupe ou à une organisation qui vous intéresse sur le campus ou en résidence vous procurera beaucoup de plaisir et de satisfaction.

Vous commencez probablement à comprendre le message : les études postsecondaires présentent un environnement propice au développement de compétences en lien avec le marché du travail. Même si vos cours n'offrent pas d'occasions de travailler en équipe ou de faire des présentations orales, vous pouvez en trouver ailleurs sur le campus. Ça vous donne une idée de la façon dont vous pouvez créer des débouchés pour vous-même?

Vous vous dites peut-être que les études postsecondaires représentent beaucoup de travail : les cours et tout ce qui va avec, les activités parascolaires et de bénévolat et, peut-être, un emploi à temps partiel. Ça l'est en effet si vous voulez sérieusement trouver un bon emploi après avoir obtenu votre diplôme. La bonne nouvelle ? Si vous suivez des cours qui vous plaisent et participez à des activités qui vous tentent, vous ne réaliserez même pas qu'il s'agit de travail. Vous vous amuserez, comme ce sera le cas dans votre carrière.

\section{Savoir y faire avec les chiffres}

Nos conseils n'auront malheureusement pas tous l'air aussi plaisant. Le plus difficile reste à venir. 
De plus en plus d'employeurs ont besoin d'employés qui s'y connaissent en chiffres. En d'autres termes, vous devez savoir compter. De plus en plus, les professionnels doivent être en mesure de manipuler et de comprendre de l'information numérique. À plus grande échelle encore, tout citoyen doit connaître les rudiments des mathématiques pour prendre des décisions éclairées sur ses investissements, ses prêts, son compte de banque, et pour comprendre le monde qui les entoure.

Certains des cours que vous suivrez vous permettront de le faire, en particulier ceux qui comportent du travail en laboratoire. Certains programmes d'études offrent cependant moins d'occasions à cet égard, mais vous aurez toujours la chance d'améliorer vos compétences.

Les étudiants des programmes de sciences sociales et humaines essaient généralement d'éviter les cours qui impliquent beaucoup de chiffres et de calculs. C'est compréhensible: les cours quantitatifs (comme les cours de statistiques et de méthodes de recherche) sont exigeants et intimidants.

La bonne nouvelle, c'est qu'il est en fait difficile d'échouer ces cours (pratiquement tous les étudiants qui persévèrent jusqu'à la fin réussissent) et qu'ils procurent des compétences utiles sur le marché du travail et dans la vie en général. Être en mesure de dire à un employeur potentiel, pendant une entrevue, que vous avez des compétences quantitatives de base ou des aptitudes en recherche jouera toujours en votre faveur. Il n'est toutefois pas nécessaire de vous inscrire à un cours de ce genre pour améliorer vos compétences relatives aux chiffres. Vous trouverez probablement des cours gratuits sur le campus pour apprendre à utiliser Excel ou un programme similaire, et des tutoriels à ce sujet sur YouTube, ou encore demandez à un ami ou à un camarade de classe de vous aider. Les gens adorent montrer à quel point ils savent compter!

L'utilisation de logiciels de tableurs ou de logiciels de statistiques plus avancés, comme Statistical Package for the Social Sciences (SPSS), Statistical Analysis System (SAS) et d'autres outils d'analyse de données, n'est pas aussi douloureuse que vous pourriez le croire, même si vous vous considérez archinul en mathématiques. Le truc, pour plusieurs, c'est de structurer l'apprentissage à partir d'un problème particulier ou d'une tâche spécifique. Essayez de prévoir vos notes. En êtes-vous capable? Pouvez-vous calculer rapidement votre moyenne à l'aide d'Excel ou d'un logiciel similaire? Pouvez-vous déterminer combien d'argent vous devrez investir? 
Beaucoup de travaux de session ou de devoirs seront bonifiés par l'ajout d'un tableau ou d'un graphique. Consultez vos manuels pour savoir comment les graphiques et les tableaux y sont utilisés pour illustrer un argument, au lieu de l'expliquer en un long paragraphe, et utilisez la même stratégie. Vous serez étonné de voir à quel point vos professeurs seront impressionnés, surtout s'ils ne sont pas particulièrement doués pour les chiffres.

Les chiffres sont tellement mystérieux pour certains qu'atteindre n'importe quel niveau d'expertise en la matière en vaudra la peine. Développer cette compétence peut être gratifiant (irons-nous jusqu'à dire amusant?). Un graphique, un diagramme ou un tableau améliore souvent la note d'une dissertation ou d'un devoir. Et, sans aucun doute, la capacité à créer un graphique, un diagramme ou un chiffrier simple joue un rôle quand vient le moment de décider qui est embauché et qui ne l'est pas. Chose sûre : la majorité de vos patrons, de vos collègues et de vos clients s'attendront à ce que vous soyez à l'aise avec les chiffres.

Savoir compter un tant soit peu pourrait donc avoir les plus grandes retombées sur votre future carrière. Ce n'est pas un hasard si les directeurs généraux et les cadres supérieurs de grandes sociétés ont souvent étudié la comptabilité et la gestion, après avoir fait des études en sciences sociales ou humaines.

\section{La prise de notes}

Vous pensez peut-être que prendre des notes en classe ou en lisant des manuels ne s'applique qu'aux études postsecondaires. Eh bien, détrompez-vous, car c'est tout le contraire! La prise de notes fait partie intégrante d'à peu près tous les emplois professionnels que vous pouvez nommer.

Par exemple, les travailleurs sociaux, les policiers, les banquiers, les professionnels de la santé et les avocats doivent prendre des notes détaillées sur leurs clients ou sur les événements. Leurs notes peuvent devenir des éléments de preuve cruciaux lors d'un procès, parfois des années après avoir été rédigées. Les enseignants prennent des notes sur les progrès que font leurs élèves et les incluent dans leur bulletin. Le travail des journalistes et autres écrivains dépend de leur capacité à prendre des notes. Les conseillers financiers prennent en note les préférences de leurs clients et les conseils qu'ils leur ont donnés, et doivent être en mesure de se souvenir rapidement de ces informations. 
Quelqu'un prendra des notes à chaque réunion d'affaires ou gouvernementale importante. Au cours de vos premières années sur le marché du travail, vous devrez peut-être préparer les procès-verbaux de différentes réunions. L'un des auteurs de ce livre a commencé sa carrière administrative en prenant des notes lors des réunions du vice-président d'une grande organisation.

Soyez assuré qu'on vous demandera souvent, au cours de votre carrière, «d'assister pour moi à la réunion de demain et de prendre des notes pour que je sache ce qui s'y est dit » ou de «lire ce rapport et m'en faire un résumé ». Si vous le faites bien, on vous remarquera et on vous récompensera. Si vous le faites mal, on ne fera que vous remarquer, et pas pour les bonnes raisons.

Vous trouverez, dans n'importe quel bureau de professionnels, des tiroirs ou des dossiers électroniques remplis de notes prises lors de cours de développement professionnel, d'observations sur le rendement des employés, de remarques sur le travail à faire, une variété de lettres et de documents ainsi que des rapports de progrès sur les projets ou les activités en cours.

Pour prendre de bonnes notes dans le monde professionnel, il faut être clair, concis et précis, soit exactement ce qu'il faut maîtriser quand on prend des notes en classe. La prise de notes en classe est toutefois plus facile parce que personne d'autre que vous ne les lira, alors que, sur le marché du travail, d'autres que vous les utiliseront souvent.

Quand vous prenez des notes en classe ou lors d'une réunion, essayez :

- de résumer l'information complexe de manière succincte ;

- d'organiser l'information pour qu'elle ait du sens (le cours ou la réunion étaient peut-être désorganisés, mais vos notes ne doivent pas l'être) ;

- de faire ressortir les idées principales et de clarifier leur signification ;

- d'incorporer et d'intégrer les faits et les concepts à des thèmes ou à des idées générales ;

- d'inclure le point de vue de plusieurs sources (de l'orateur, des lectures, des questions posées, des événements passés);

- d'identifier les sections et les problèmes qui requièrent d'explorer un peu plus avant le sujet.

Votre technique de prise de notes laisse à désirer si :

- vous essayez de tout prendre en note (il n'y a aucune raison de le faire puisque les notes sont des résumés); 
- vous n'établissez pas de contact visuel avec l'orateur (continuez de lui porter attention pendant que vous écrivez) ;

- vous n'avez pas de questions ou de sujets qui requièrent d'être développés (si possible, posez des questions au présentateur pour clarifier certains points);

- vos notes n'ont aucun lien entre elles ou se présentent sous forme de liste à puces, qui n'est d'aucune utilité.

Les techniques qui vous permettront d'améliorer votre capacité à prendre des notes en classe sont les suivantes:

- faites les lectures avant le cours;

- créez votre propre code d'abréviations pour vous souvenir de connexions ou de questions importantes (comme =, ?, », N.B. ; N.B. est l'abréviation de nota bene ou notez bien!) ;

- relisez vos notes alors qu'elles sont encore fraîchement prises et faites des connexions ou développez ce que vous n'avez pas eu le temps d'écrire pendant le cours ;

- répondez aux questions que vous avez prises en note, en consultant les lectures, les autres étudiants ou le professeur ;

- résumez régulièrement vos notes sur un sujet précis ou sur une section du cours;

- comparez vos notes avec celles des autres étudiants dans votre groupe.

Nous enseignons, depuis longtemps, à de grands groupes d'étudiants. Nous pouvons donc affirmer avec une certaine autorité que la capacité à prendre de bonnes notes de cours fait une énorme différence entre les étudiants qui réussissent bien dans leurs évaluations et ceux qui éprouvent des difficultés. Plusieurs étudiants prennent des notes à l'aide de leur ordinateur; dans certains cours, les ordinateurs sont obligatoires. Toutefois, nous recommandons fortement d'utiliser un crayon et du papier. La raison en est bien simple : avoir un ordinateur ou une tablette entre les mains incite à faire plusieurs choses à la fois : chercher de l'information en ligne, jouer à des jeux vidéo, regarder des films ou se balader sur les réseaux sociaux. Faire plusieurs choses à la fois pendant les cours, quoique tentant, donne de moins bonnes notes. Vous ne nous croyez pas? Consultez les études qui comparent les étudiants qui prennent des notes à la main avec ceux qui tapent leurs notes directement sur leur portable. Les étudiants qui prennent leurs notes à l'ordinateur obtiennent des notes $17 \%$ plus basses aux tests, soit la différence entre un $\mathrm{B}+$ et un $\mathrm{B}^{1}$. 
Par ailleurs, être assis au centre ou à l'avant de la salle de classe, le téléphone et l'ordinateur éteints, est plus propice à la prise de bonnes notes et à la réussite. S'asseoir à l'arrière de la classe est mauvais pour l'apprentissage et utiliser un téléphone vous distrait d'une activité qui devrait avoir toute votre attention. Prenez l'habitude de vous asseoir dans les premières rangées et vous vous rendrez compte que l'écoute et la prise de notes deviennent plus faciles et plus agréables.

Si vous n'apprenez pas à prendre de bonnes notes pendant vos études postsecondaires, il vous sera encore plus difficile de le faire plus tard dans la vie et vous limiterez vos ambitions de carrière.

\section{S'organiser}

Dès le départ, le manque de motivation représente l'un des plus gros écueils qui nous empêchent d'exécuter nos tâches ou de faire notre travail. Encore une fois, cela met de l'avant un thème central de ce livre: vous devez choisir des cours et des emplois qui vous plaisent. Cependant, même si vous avez fait tout ce qu'il faut, se mettre au travail peut parfois être difficile.

On se rend tous coupables de procrastination à un moment ou à un autre, par distraction ou parce qu'on se tourne les pouces au lieu d'apprendre. Vous vous imaginez peut-être qu'il nous a été facile d'écrire ce livre parce que nous sommes professeurs et que nous croyons fermement au sujet. Pas du tout!

Vous trouverez ci-dessous des trucs qui nous ont aidés et qui vous aideront à persévérer quand vous préféreriez ne pas travailler. Ils s'appliquent autant aux études qu'au travail.

\section{Se fixer des objectifs à long terme et des objectifs à court terme}

De la même façon que vous avez besoin de raisons et d'objectifs pour vous mettre en forme ou apprendre un nouveau sport, vous devez vous motiver à entreprendre une activité en lien avec vos études, que ce soit étudier pour un test, rédiger une dissertation ou vous attaquer à autre chose. Vos objectifs généraux à long terme peuvent être aussi abstraits que ceux de devenir un bon orateur, de contribuer à la société ou de combler les attentes raisonnables de vos parents ou autres personnes importantes dans votre vie.

Vos objectifs à plus court terme devraient être assez précis pour vous récompenser et vous garder motivé. Beaucoup d'étudiants 
font de leur carrière leur objectif à long terme et de l'obtention de bonnes notes leur objectif à court terme. Cette approche est tout à fait acceptable tant et aussi longtemps que vous êtes conscient que vos bonnes notes doivent elles aussi être divisées en plusieurs objectifs à court terme.

L'obtention de bonnes notes implique normalement:

- de bien étudier la matière ;

- de faire tous les devoirs obligatoires et facultatifs ;

- d'assister à tous les cours et de prendre de bonnes notes;

- de travailler en groupe ou, à tout le moins, de réviser vos notes et de faire les lectures avec d'autres étudiants ;

- de faire preuve d'initiative en allant au-delà de ce qui est demandé, que ce soit dans vos lectures, vos recherches ou vos devoirs écrits.

$\mathrm{Si}$ vous atteignez ces objectifs à court terme, récompensez-vous régulièrement. Vous l'avez bien mérité! En divisant vos objectifs à long terme en objectifs à court terme, vous éviterez de vous poser la question paralysante que se posent beaucoup d'étudiants et qui mène à la procrastination : "J'ai tellement de choses à faire ; qu'est-ce que je devrais faire en premier?»

\section{Gérer son temps}

Les étudiants font souvent les choses à la dernière minute, non pas par paresse, mais parce que c'est une façon rapide et facile de s'organiser et de se motiver. Samuel Johnson (1709-1784), écrivain du XvıII siècle et inventeur du dictionnaire, a un jour observé qu'une personne peut être vraiment concentrée la veille de son exécution. Certains étudiants semblent partager ce point de vue et iront même jusqu'à dire qu'ils font un travail de meilleure qualité la veille de la date de remise. À l'instar des professionnels qui ont du succès, les professeurs et les étudiants d'expérience savent que c'est faux. Voudriez-vous que votre chirurgien vous opère s'il est ultra-stressé ou épuisé et qu'il carbure au café? Voudriez-vous être représenté par un avocat qui n’a pas dormi la veille de votre audience en cour et qui est incapable de se concentrer?

Certains arrivent mieux que d'autres à gérer leur temps. Il se peut que leur cerveau soit fait autrement ou qu'ils aient acquis des habiletés de bonne gestion du temps par osmose avec leurs parents ou leur douce moitié. Il reste que la plupart d'entre nous avons besoin 
de planifier notre temps et de s'en tenir à un horaire. Le temps est une denrée rare quand on est étudiant et c'est pourquoi il est important de bien l'utiliser.

Voici quelques trucs pour éviter de perdre du temps:

- Forcez-vous à suivre une routine. Essayez de vous lever à la même heure chaque matin et de vous coucher à la même heure chaque soir. Selon des experts en planification, établir une routine quotidienne est la meilleure façon de tirer parti du temps passé sur les tâches et de maximiser les accomplissements ;

- Faites un horaire pour la session et inscrivez-y toutes les dates de remise de travaux et d'examens;

- Calculez combien de temps vous aurez besoin pour vous préparer en partant de la date de remise et en comptant à reculons. Estimez honnêtement le nombre d'heures que vous devrez consacrer à chaque activité pour réussir. La plupart des cours, au grand dam des étudiants, comprennent plus de travaux vers la fin de la session. Minimisez les effets de cette période occupée en planifiant votre temps et en commençant à travailler bien avant la date limite ;

- Déterminez les priorités. Certains sujets ou devoirs sont plus importants que d'autres. Passer trop de temps sur une tâche sans importance est une erreur que commettent souvent les étudiants et les professionnels inexpérimentés. Vous pouvez mettre en relief les différences de priorité en utilisant des polices de caractères différentes ou des couleurs variées. Soyez toujours prêt à ajuster vos priorités au fur et à mesure que la session avance ;

- Utilisez des planificateurs pour vos devoirs et vos semaines (voir l'exemple à la page 39). À la fin de chaque semaine ou de chaque mois, prenez une heure pour revoir ces planificateurs et apportez-leur les ajustements nécessaires ;

- Visez une amélioration constante au lieu d'espérer atteindre la perfection. Ce conseil est particulièrement pertinent pour les étudiants qui viennent de commencer à développer une compétence ou une habileté précise. Vous serez assurément nul la première fois que vous essayerez quelque chose. C'est la vie. Ce qui compte, c'est de s'améliorer avec le temps. 
PLANIFICATEUR DE TRAVAUX

Date limite de remise :

\begin{tabular}{|l|c|l|l|}
\hline Composante & Nombre d'heures & Date butoir & Fait \\
\hline Recherche & 4,5 & & \\
\hline $\begin{array}{l}\text { Trouver l'hypothèse } \\
\text { de départ/question }\end{array}$ & 1 & & \\
\hline Ébauche de structure & 3 & & \\
\hline Composition & 7 & & \\
\hline Documentation & 2 & & \\
\hline Révision & 3 & & \\
\hline Travail terminé & 20,5 & & \\
\hline
\end{tabular}

PLANIFICATEUR HEBDOMADAIRE

Semaine du

\begin{tabular}{|l|c|l|c|c|}
\hline Activité & Objectif & \multicolumn{1}{|c|}{ Composante } & Nombre d'heures & Fait \\
\hline $\begin{array}{l}\text { Examen de } \\
\text { statistiques }\end{array}$ & B & Relire textes & 3 & \\
\hline & & Relire notes & 1 & \\
\hline & & Graphiques et tableaux & 1,5 & \\
\hline & & Rencontre groupe d'étude & 2 & \\
\hline Sociologie & B + & Lire chapitres 6 et 7 & 2,5 & \\
\hline & & $\begin{array}{l}\text { Répondre aux questions du } \\
\text { chapitre }\end{array}$ & 1 & \\
\hline Psychologie & A & Aller au labo & 1,5 & \\
\hline & & Lire chapitre 5 & 1,5 & \\
\hline & & Séance de questions et réponses & 1 & \\
\hline Histoire & B & Recherche en bibliothèque & 3,5 & \\
\hline
\end{tabular}

\section{Garder le cap}

Les planificateurs pour les devoirs et les semaines aident les étudiants à garder le cap. En planifiant à reculons, vous pouvez établir un calendrier qui vous permettra de terminer un devoir à temps. Les planificateurs deviendront encore plus efficaces au fur et à mesure que vous découvrirez combien de temps vous mettez à faire les différentes parties d'un devoir.

Certains étudiants trouvent utile d'inclure des récompenses dans leur liste d'activités, comme une soirée au pub ou un film et de la pizza avec des amis. 


\section{Gérer les distractions de manière efficace}

Le philosophe existentialiste français Jean-Paul Sartre (1905-1980) a écrit, un jour, que "l'enfer, c'est les autres. » Quand vous essayez de travailler et que vos amis, vos parents, vos frères et sœurs, ou les autres étudiants veulent vous parler ou vous proposer une activité, vous pourriez être tenté d'accepter. Vous pouvez minimiser ces interruptions en éteignant votre téléphone et en étudiant à la bibliothèque, mais vous ne pourrez jamais les éliminer complètement.

La plupart des gens ne comprennent pas, ne se souviennent pas ou ont préféré oublier à quel point les interruptions sont dérangeantes. Ils peuvent même être insultés si vous leur dites souvent «désolé, mais je suis en train de travailler sur un projet. Je t'appelle plus tard. » Votre façon de gérer ces interruptions peut modeler votre expérience étudiante, voire votre carrière. Les étudiants et les employés qui arrivent à marier fermeté et délicatesse dans de telles situations ont généralement plus de succès, gagnent le respect des autres et parviennent à devenir des leaders.

Tout le monde possède un niveau d'intelligence semblable. Ceux qu'on qualifie de doués ou de brillants ne sont généralement pas très différents des autres. Ils ont seulement appris à se concentrer sur le problème en question et à lui donner toute l'attention qu'il mérite. Pour ce faire, ils réduisent au minimum les interruptions.

Voici quelques trucs pour réduire les distractions tout en gardant vos amis :

- Dites-leur quand vous êtes occupé et quand vous êtes disponible ;

- Restez ferme. Ils comprendront que vos études sont importantes pour vous;

- Soyez constant. De cette façon, vos amis comprendront la situation et ne se sentiront pas rejetés ;

- Communiquez avec eux quand vous êtes libre ;

- Accordez-leur toute votre attention quand vous êtes avec eux. De cette façon, ils en viendront à apprécier la qualité du temps que vous passez ensemble, au lieu de la quantité.

Liez-vous d'amitié avec des gens dont les objectifs et les buts ressemblent aux vôtres et qui reconnaissent l'importance de la concentration. Vous pouvez même combiner interactions sociales et réussite scolaire en formant un groupe d'étude. Certaines amitiés incroyables et des réseaux de contacts futurs naissent souvent d'un groupe 
d'étude. Les études postsecondaires vous offrent la chance de rencontrer de nouvelles personnes et de vous faire de nouveaux amis. Les amitiés qui se créent au cours de vos études durent souvent toute une vie. Un problème se pose uniquement si vous laissez votre vie sociale prendre le dessus sur votre vie d'étudiant. Ceux qui arrivent à trouver un équilibre entre leur vie sociale et leur vie étudiante réussissent généralement très bien, non seulement dans leurs études, mais par la suite. D'un autre côté, trop favoriser leur vie sociale en mène plusieurs à abandonner les études. C'est très tentant de se laisser aller, surtout après s'être libéré des contraintes de l'école secondaire et des parents. C'est pourquoi il est particulièrement important pour ceux qui quittent la maison de développer une force de caractère qui leur permettra de bien réussir dans leurs études. Si vous n'avez pas développé cette qualité au moment d'entrer sur le marché du travail, vous aurez beaucoup de mal. Si vous avez un bon sens de l'organisation et si vous êtes capable de vous concentrer, vous disposez déjà d'une longueur d'avance sur vos pairs.

\section{Note}

1. Voir Fox News, «Students' Use of Laptops in Class Lowers Grades: Canadian Study », 16 août 2013, http://www.youtube.com/watch?v=O2aybVf5QAM. Les résultats sont tirés de Faria Sana, Tina Weston et Nicholas J. Cepeda, « Laptop Multitasking Hinders Classroom Learning for Both Users and Nearby Peers », Computers and Education, vol. 62, mars 2013, p. 24-31, http://ac.els-cdn.com/ S0360131512002254/1-s2.0-S0360131512002254-main.pdf?_tid=c12dccd6-3e6411e7-963a-00000aacb362\&acdnat=1495398991_77bd26a2da81a924e14f79409838e 8 aa. 
Page blanche conservée intentionnellement 


\section{Réussir en classe et au travail}

ous vous expliquerons dans ce chapitre comment bien réussir
vos examens, rédiger des dissertations et produire des rapports tout en vous préparant à faire face aux mêmes défis au cours de votre carrière. La plupart des étudiants passent, en effet, énormément de temps à préparer des examens et à rédiger des dissertations et autres travaux (comme des rapports de laboratoire). Pourquoi ne pas vous servir de cette expérience pour réussir dans le monde du travail ?

Passer des examens, rédiger des dissertations et faire des rapports sont de très bons moyens de vous exercer à passer tous les tests qui vous attendent au cours de votre carrière et pour lesquels vous ferez beaucoup de recherches et de rédaction. En fait, les examens que vous aurez dans la vraie vie auront de bien plus graves conséquences que ceux que vous ferez pendant vos études. Si vous échouez à un examen, votre moyenne baissera ou, dans le pire des cas, vous échouerez le cours. Rater un examen sur le marché du travail pourrait vous coûter votre emploi ou, à tout le moins, ruiner vos chances d'avancement (et d'obtenir l'augmentation de salaire qui l'accompagne).

Commençons donc par les examens. 


\section{Les examens, ça fait partie de la vie}

Vous pensez peut-être que la capacité à passer des examens est ce qu'il y a de moins transférable comme compétence et vous vous imaginez que vous n'aurez plus à en faire une fois que vous aurez obtenu votre diplôme. Détrompez-vous! La plupart des professionnels doivent subir encore beaucoup d'examens après avoir terminé leurs études postsecondaires.

Il n'est pas rare que des tests écrits fassent partie des demandes d'emploi. En fait, plusieurs emplois dans les secteurs public et privé - et pas seulement ceux auxquels on pense automatiquement, comme les emplois de policier et de pompier - exigent des candidats qu'ils réussissent une panoplie d'examens avant même d'être convoqués en entrevue.

Même si une demande d'emploi pour un poste professionnel ne comporte pas de composante écrite, l'entrevue elle-même est un test, donc une sorte d'examen, pour vérifier ce que vous avez appris et comment vous êtes capable d'organiser et de présenter vos idées et vos pensées. Bien réussir en entrevue sera hors de votre portée si vous n'arrivez pas à vous souvenir d'informations importantes, à répondre à des questions inattendues et à penser de façon analytique.

Plusieurs professionnels, comme les avocats, les comptables, les ingénieurs, les physiothérapeutes et les conseillers financiers, doivent passer des examens de qualification et de compétence. Vous-même devrez probablement suivre des cours, subventionnés par votre employeur ou non, pour acquérir des qualifications précises ou en apprendre davantage sur de nouveaux développements dans votre domaine. Ces cours comportent généralement de très longs tests.

Si vous êtes à l'université, vous déciderez peut-être de poursuivre vos études après avoir terminé votre baccalauréat, en étudiant le droit ou en faisant un MBA ou tout autre programme de maitrise. Plusieurs de ces programmes exigent des candidats qu'ils passent un examen d'entrée, comme le Law School Admission Test (LSAT) pour le droit, le Graduate Management Admission Test (GMAT) ou le Test d'aptitude aux études de gestion et au management des entreprises (TAGE MAGE) pour la gestion ou le Graduate Record Examination (GRE) pour les études supérieures. Consultez le chapitre 11 pour plus de renseignements sur les études supérieures.

De plus, vous serez quotidiennement confronté à des tests au travail. Par exemple, votre patron pourrait vous demander de décrire les 
problèmes sous-jacents à votre plus récent projet ou à une entente que vous avez conclue. Ou bien, un client pourrait vous téléphoner pour que vous lui expliquiez pourquoi le rendement de ses actions n'est pas celui que vous lui aviez laissé entendre, il y a deux ans. Il n'a rien à faire de vos excuses et, si vous n'êtes pas en mesure de répondre à ses questions, il ira voir ailleurs. Ou encore, le père de Sabrina pourrait se pointer dans votre salle de classe sans préavis pour discuter des progrès de sa fille. Si vous réussissez de tels tests, vos collègues et votre patron vous lanceront des regards approbateurs. Si vous bredouillez et pataugez misérablement sans arriver à vous en sortir, votre carrière en souffrira.

\section{Réduire l'anxiété}

C'est un fait: les examens sont anxiogènes. S'ils ne vous rendent pas nerveux, nous avons une mauvaise nouvelle pour vous: vous êtes probablement mort. Il importe peu que tout le mauvais sang que vous vous faites à leur sujet soit complètement démesuré par rapport au poids des examens comme tel. Vos émotions vous définissent et vous ne pouvez pas toujours les contrôler. Heureusement, vous êtes aussi défini par ce que vous savez. Si vous arrivez à vous calmer les nerfs un peu et à vous servir de l'examen pour faire la preuve de votre savoir, vous avez de bonnes chances de sortir vivant de la salle d'examen.

Le simple mot « examen » fait naître un sentiment de terreur dans le cœur de plusieurs étudiants. Des diplômés, des années après avoir terminé leurs études, font parfois des cauchemars dans lesquels ils errent sans fin à la recherche de leur salle d'examen. Un professeur n'a qu'à chuchoter «ceci pourrait se retrouver dans l'examen », pour que les centaines d'étudiants qui remplissent l'auditorium lui portent soudainement attention. Des candidats au doctorat ont maintes fois fondu en larmes en se préparant à leurs examens. On pourrait s'attendre à ce qu'ils soient à l'aise avec les examens, étant donné que ça fait une éternité qu'ils sont aux études. Pas du tout.

Vous ne pourrez jamais éliminer complètement l'anxiété causée par les examens ou la peur de ne pas bien réussir, que ce soit à l'école ou au travail. Vous pouvez cependant la diminuer. Commencez à développer vos techniques de gestion de l'anxiété alors que vous êtes toujours aux études pour être en mesure de les appliquer quand vous serez sur le marché du travail.

Voici les façons les plus efficaces de diminuer l'anxiété reliée aux examens : 
- Sachez de quoi vous parlez. Maîtrisez bien le matériel sur lequel porte le test ou l'examen. Plus précisément, assurezvous de comprendre les idées ou les thèmes clés du cours et comment les faits s'y rapportent;

- Tirez profit de ce que le stress peut avoir de positif. Sans stress, nous n'aurions pas de raisons de faire de notre mieux. La capacité à bien fonctionner en situation de stress constitue souvent ce qui sépare les cadres supérieurs des employés ordinaires, et les étudiants forts des étudiants moyens ;

- Débarrassez-vous du stress excessif. Ne le laissez pas vous paralyser. Divisez votre préparation à l'examen en étapes. Quand vous n'en pouvez plus, allez faire de l'exercice, regardez un film ou sortez pour la soirée. Sachez quand éviter les distractions et quand il est productif de vous distraire. Certaines compagnies progressistes disposent de piscines et de tables de ping-pong dans des salles de repos où les employés peuvent relaxer et refaire le plein d'énergie ;

- Ne vous bourrez pas le crâne à la dernière minute. On sait, on sait: ça fonctionnait à l'école secondaire, n'est-ce pas? Eh bien, ça ne fonctionnera pas ici ni sur le marché du travail ;

- S'il faut absolument que vous vous bourriez le crâne, faitesle intelligemment. Remplissez votre mémoire à court terme de faits élémentaires, de noms et de dates, mais n'essayez pas d'apprendre les concepts ou les idées clés à la dernière minute ;

- Vivez le moment présent et demeurez concentré. C'est ici que coïncident les approches du maître zen et du bon étudiant. Il est impossible d'être stressé si vous vivez le moment présent et demeurez concentré. Trouvez du plaisir à l'expérience de l'examen. Et avant de nous demander d'arrêter de dire n'importe quoi, répondez à cette question: quand vous sentez-vous le plus stressé ? Quand vous étudiez pour l'examen ou quand vous vous assoyez et commencez à écrire? Il y a de bonnes chances que votre stress disparaisse dès l'instant où vous vous concentrez sur ce que vous avez à faire. Vivez le moment présent. Réjouissez-vous d'avoir maintenant quelque chose de mieux à faire et de plus pressant que de vous inquiéter ;

- Mettez les choses en perspective. Un examen n'est pas une question de vie ou de mort. Croyez-le ou non, beaucoup, beaucoup de gens ont déjà échoué à des examens et ont tout de même réussi à avoir une brillante carrière. L'un des 
auteurs de ce livre a complètement échoué sa première année d'université (un indice : ses initiales sont J.A.D.) et l'autre, son cours d'histoire de première année!

\section{Se préparer pour un examen important}

Les examens ne sont pas tous importants. Vous perdez votre temps si vous vous en faites au sujet de petits tests ou si vous passez des heures à vous préparer pour un examen dans un cours facile que vous réussissez déjà les doigts dans le nez. Par contre, pour les gros examens, ceux qui font vraiment peur, ou pour les moments qui font ou ruinent une carrière, il est important d'avoir une stratégie personnelle. En voici une qui fonctionne pour certains et qui peut être adaptée à votre situation personnelle:

- Ne vous mettez pas trop de pression. Donnez-vous comme objectif de bien faire, au lieu de viser une note précise. Si vous êtes perfectionniste, laissez de côté votre besoin d'être parfait pour le moment ;

- Ne vous préoccupez pas de l'avenir. Plus précisément, oubliez les répercussions que pourrait avoir l'examen sur votre avenir. Concentrez-vous sur le moment présent. Si vous voulez absolument voir plus loin, pensez à votre soulagement quand l'examen sera terminé ;

- Concentrez-vous. Préparez-vous pour l'examen au lieu d'y penser. La préparation à un examen ne fait pas peur; elle est seulement très ennuyante ;

- Lamentez-vous comme s'il n'y avait pas de lendemain. Dites à vos amis que vous vous préparez à un examen et que vous n'aurez plus de vie sociale (ou de vie tout court) jusqu'à ce que celui-ci soit fini. Ils comprendront et vous laisseront tranquille, ce qui vous permettra de vous concentrer sur votre préparation. De toute façon, vous serez tellement chiant avec vos lamentations qu'ils ne voudront pas passer de temps avec vous;

- Organisez ce que vous devez étudier et rendez votre espace propice à l'étude. Cela pourrait devenir un rituel formel pour vous aider à vous y mettre. Ne laissez toutefois pas l'organisation prendre le dessus sur l'étude. Certains jeunes cadres ne grimpent jamais les échelons parce qu'ils passent plus de temps à faire le ménage de leur bureau qu'à s'assurer qu'ils 
termineront le projet de l'année à temps ;

- Prenez des pauses, que vous en ayez besoin ou non. L'étude est une activité qui prend fin seulement quand l'examen commence. Vous devez donc planifier des pauses régulières pendant que vous étudiez;

- Cernez vos lacunes dès le début. N'attendez pas à la dernière minute pour découvrir que vous ne saisissez pas certains éléments essentiels. Il sera alors trop tard ;

- Retranscrivez vos notes. Relire vos notes est loin d'être la meilleure façon de vous préparer à un test. Par contre, si vous les retranscrivez, vous retiendrez mieux les idées et l'information et ferez des connexions qui vous avaient échappé auparavant.

On sous-estime souvent l'importance d'avoir les idées claires et de relaxer pour réussir ses examens haut la main et pour se lancer dans des projets qui nécessitent de penser de façon critique et créative.

Voici quelques trucs qui vous permettront de relaxer:

- N'apprenez pas la matière par cœur; appliquez-vous plutôt à la comprendre. La mémorisation est une des pires façons d'organiser l'information. Les bons examens n'évaluent pas votre capacité à mémoriser la matière, mais visent plutôt à déterminer à quel point vous la comprenez. Vous verrez peutêtre votre niveau d'anxiété diminuer quand vous vous rendrez compte que vous n'avez plus à autant mémoriser la matière ;

- Soyez ouvert d'esprit. Si, pour vous, un problème n'a qu'une seule solution et qu'au travail, vous n'arrivez pas à déroger de vos solutions toutes faites, vous manquerez de flexibilité et vous vous perdrez dans les détails. Une meilleure approche consiste à vous assurer de bien comprendre la situation et d'être ouvert d'esprit pour pouvoir faire face à toute éventualité ;

- Assurez-vous d'avoir une bonne nuit de sommeil. La veille de l'examen, dites-vous que vous avez fait tout votre possible et que tout ce qui vous reste à faire, c'est de bien dormir pour concentrer toutes vos énergies sur l'examen.

\section{Le jour J}

Voici quelques trucs pour vous aider à bien vous en sortir le jour de l'examen :

- Assurez-vous d'avoir tout ce dont vous avez besoin, y compris des stylos et des crayons supplémentaires ; 
- Arrivez tôt à la salle d'examen ;

- Regardez autour de vous. Vous voyez comment tout le monde est nerveux? Vous n'êtes pas le seul à souffrir ;

- Réjouissez-vous: ce moment pénible et douloureux sera bientôt chose du passé;

- Quand vous recevez l'examen, relaxez et prenez le temps de le lire du début à la fin. Ne commencez pas à y répondre tout de suite ;

- Portez attention à l'échelle de notation. Repérez les questions qui valent le plus de points et assurez-vous de ne pas passer trop de temps sur une partie au détriment d'une autre ;

- Soyez stratégique. Par exemple, donnez-vous comme objectif de répondre à toutes les questions de l'examen au lieu d'obtenir des notes parfaites pour certaines questions ;

- Concentrez-vous sur les questions. Lisez-les attentivement. Lisez-les au moins deux fois. Puis relisez-les.

Transposés à un environnement de travail ou à une réunion, pouvoir se concentrer sur la tâche à accomplir, être préparé, focaliser sur ce qui est réalisable et user de stratégies représentent un avantage non négligeable.

\section{Stratégies pour réussir les examens à choix multiples}

Il y a peu d'examens à choix multiples dans le monde du travail, ce qui, selon nous, suggère qu'ils ne représentent pas la meilleure façon de vérifier la compréhension d'une personne ou de donner une idée du potentiel de celle-ci. Nous doutons également que la réalité corresponde vraiment au modèle noir et blanc des scénarios à choix multiples. Malheureusement, il y a beaucoup d'examens à choix multiples dans le monde professionnel de l'éducation et dans plusieurs domaines d'études postsecondaires. Aussi, quelle que soit la validité éducative des examens à choix multiples, vous feriez mieux de vous préparer à en passer.

Certains étudiants préfèrent les questions à choix multiples aux questions à développements. Le désavantage d'un examen à choix multiples est toutefois que le professeur possède le contrôle absolu sur la façon dont les étudiants doivent démontrer qu'ils connaissent la matière. Habituellement, il n'y a qu'une seule bonne réponse, sans possibilité de la nuancer. De plus, ces examens sont remplis de questions pièges, car elles permettent au professeur de déterminer si les 
étudiants ont bien lu les questions et en ont repéré les subtilités.

Il est plus difficile d'étudier pour un examen à choix multiples que pour tout autre examen. Les examens à choix multiples :

- exigent une compréhension précise de la matière et pas seulement une solution raisonnable à un problème ;

- mêlent les concepts du cours de manière à embrouiller;

- reformulent les concepts pour vous forcer à réfléchir sur d'infimes distinctions;

- jouent avec des détails, des analogies et des comparaisons qui prennent du temps à déchiffrer;

- vous obligent à penser à la fois à la structure et à la signification de la question.

Puisque vous devrez jongler avec plusieurs variables pendant la courte période de temps que durera l'examen, il est essentiel pour vous d'avoir une stratégie claire pour les aborder. Il est particulièrement important de savoir pourquoi les étudiants réussissent moins bien ce genre d'examen: ils lisent les questions trop rapidement, restent tout de suite coincés sur une ou deux d'entre elles et planifient mal leur temps. Voici quelques conseils pour éliminer ou réduire grandement ces problèmes :

- Répondez d'abord aux questions faciles. Mettez en banque les points des questions faciles avant de vous attaquer à quoi que ce soit d'autre. De cette façon, vous vous sentirez bien et en confiance, car vous aurez choisi les bonnes réponses pour la plupart de ces questions. Si vous passez trop de temps sur les questions difficiles, vous risquez de laisser échapper des points qui, autrement, seraient gagnés d'avance ;

- Lisez attentivement les questions difficiles. Encerclez les mots clés. Essayez de trouver la réponse avant de regarder les choix qui vous sont proposés et avant de (probablement) vous embrouiller ;

- Vérifiez ensuite les choix. Ils déclenchent souvent une réponse. Par exemple, si vous savez que trois réponses sont incorrectes, celle qui reste doit être la bonne. Certains examens comprennent des questions dont les réponses n'ont jamais été données en classe et le professeur s'attend à ce que vous éliminiez les choix que vous devez savoir être faux. Plutôt sournois, n'est-ce pas?

- Si vous ne savez pas la réponse, passez à la question suivante. Vous voudrez peut-être bûcher sur la question, et c'est normal, 
mais il est parfois préférable d'abandonner et de passer à la question suivante que de rester coincé. Choisissez vos combats ;

- Essayez de repérer des tendances et des similitudes. Vous devez évidemment le faire à chaque question afin de trouver la bonne réponse, mais faites-le également entre les questions. Il arrive souvent, dans un examen à choix multiples, que la réponse à une question donne une bonne idée de la réponse à une autre question ;

- Devinez du mieux que vous le pouvez. Il ne s'agit pas d'y aller complètement à l'aveuglette. Il faut choisir la plus probable des solutions. Assurez-vous toujours, toutefois, que vous ne perdrez pas de points si vous faites erreur. Le cas échéant, ne répondez pas et passez à la question suivante ;

- Ne changez pas les réponses aux questions dont vous avez deviné la réponse. C'est une perte de temps ;

- Faites attention aux pièges habituels. Les mots "toujours» et «jamais » signifient généralement que la réponse est trop inclusive ou trop exclusive pour être la bonne. Vérifiez s'il y a des doubles négations qui rendent positive la bonne réponse. Par exemple, une double négation comme "pas inutile» se traduit par « utile» et «pas impossible» signifie " possible»;

- Relisez vos réponses encore et encore, si vous en avez le temps. Vous trouverez assurément une mauvaise réponse, signe que vous n'avez pas bien lu la question. De plus, des réponses vous viendront à l'esprit au fur et à mesure que vous établirez des liens entre les questions.

Bien que la validité éducative des examens à choix multiples soit, à tout le moins, discutable, on les utilise souvent dans le monde de l'éducation professionnelle parce qu'ils permettent aux professeurs de classer facilement les étudiants. Si vous vous préparez à une profession ou si vous souhaitez mettre à niveau vos compétences professionnelles, vous devrez probablement faire ce genre d'examen de temps à autre. Selon nous, c'est désolant, mais c'est comme ça.

\section{Stratégies pour réussir les examens écrits}

Les examens écrits sont généralement composés de questions à courts et à longs développements. Les courts développements sont habituellement plus faciles à rédiger que les longs parce qu'ils évaluent généralement la capacité à se souvenir de faits et peuvent souvent être 
écrits sous forme de liste à puces. Les longs développements sont plus difficiles, parce qu'ils évaluent non seulement votre capacité à analyser le matériel du cours, mais aussi à en faire la synthèse de façon éloquente et sensée.

Les examens à longs développements vous prépareront cependant mieux à votre carrière professionnelle. Au travail, il ne sera pas rare qu'on vous demande, et souvent sans vraiment de préavis, d'analyser et d'expliquer un événement ou de trouver une solution à un problème. Pourquoi les ventes n'ont-elles pas augmenté? Pourquoi a-t-on commis ce crime? Qu'arrivera-t-il à notre part de marché ? Quelle devrait être notre stratégie globale? Pourquoi Sabrina a-t-elle échoué son test d'histoire?

L'avantage d'un examen à développements est qu'il vous donne beau jeu. En effet, vous décidez de la meilleure façon d'aborder la question pour mettre en valeur votre compréhension de la matière. Comme le grand joueur de soccer Stanley Matthews avait l'habitude de dire, c'est toujours mieux d'avoir le ballon parce qu'on sait exactement ce qu'on veut faire avec.

Plusieurs des stratégies pour faire un examen écrit sont les mêmes que pour un examen à choix multiples. Vous devez lire attentivement les questions, déterminer le temps que vous passerez sur chacune d'entre elles selon leur valeur et bien planifier votre temps.

De plus, vous devriez passer au moins $20 \%$ de votre temps à préparer le plan de chaque réponse. Loin de constituer une perte de temps, le plan vous permettra ensuite de composer rapidement votre réponse et de vous assurer qu'elle est bien organisée.

Il existe une autre bonne raison de faire un plan et de l'inclure dans vos réponses d'examen. Si vous ne réussissez pas à terminer votre rédaction, le plan prouvera au correcteur que vous maîtrisez le contenu du cours, que vous avez compris la question, que vous savez comment y répondre et que le manque de temps est la seule raison pour laquelle vous n'y avez pas répondu. Beaucoup de correcteurs donneront souvent une aussi bonne note au plan qu'à une réponse complètement rédigée.

Pour bien faire aux examens à développements, vous devez:

- bien comprendre les théories et les idées principales du cours ainsi que les liens entre elles ;

- savoir bien écrire (nous en parlerons plus loin dans le chapitre) ;

- comprendre la question et y répondre ! 
Pourquoi mettre un point d'exclamation ? Parce que la triste réalité, c'est que les étudiants échouent souvent aux examens à développements parce qu'ils ne portent pas attention aux termes utilisés et au but de la question.

Vous devriez vous écrire le message suivant dans le front : si vous ne répondez pas à la question, peu importe vos connaissances, peu importe la quantité de lignes que vous rédigez ou à quel point vous comprenez l'idée générale sur laquelle porte le sujet, vous obtiendrez un beau gros zéro. Rien. Nada.

Pouvoir s'attaquer au cœur du sujet est très important dans le monde professionnel. Quand votre patronne vous demande de lui rapporter ce qui s'est passé lors de la réunion à laquelle vous avez assisté pour elle, que veut-elle (et doit-elle) savoir ? Qui était présent? Qui a soulevé telle et telle problématique? Qui était en désaccord avec qui ? Pourquoi les participants ont-ils choisi telle approche au lieu de telle autre? Vous ne disposez que de cinq minutes, ou d'une page, pour lui dire ce qui s'est passé. Si vous ne lui donnez pas les réponses qu'elle cherche, elle se trouvera dans l'eau chaude avec son patron et ça, c'est généralement mauvais signe pour vous aussi !

Ou encore, que veut réellement savoir un client qui pose des questions sur ses placements? Veut-il qu'on lui explique en détail pourquoi il a perdu de l'argent? Ou cherche-t-il à s'assurer qu'il fera des profits à l'avenir? Essaie-t-il de voir si vous vous souvenez de ce que vous lui avez dit il y a deux ans ? Donnez-lui la mauvaise réponse, que ce soit en personne ou par courriel, parce que vous n'avez pas compris sa question, et vous le perdrez comme client.

Et qu'en est-il du père de Sabrina? Souhaite-t-il une analyse détaillée du test d'histoire de sa fille, question par question? Ou essaie-t-il de déterminer si vous êtes un bon enseignant? Cherchet-il à savoir autre chose? Pour répondre, vous devez commencer par comprendre sa question. Trompez-vous et votre directeur d'école commencera à s'interroger à votre sujet.

Si vous lisez mal une question à choix multiples, vous ne perdrez que quelques points. Si vous interprétez mal une question à développements dans un examen qui ne comporte que trois questions, la meilleure note possible que vous obtiendrez descend rapidement à $66 \%$. C'est pourquoi il est si important de lire attentivement les questions d'un examen à développements. En règle générale, vous devriez passer au moins dix minutes à analyser la question et à vous assurer que vous la comprenez avant de commencer à rédiger. 
Et, comme si ce n'était pas assez compliqué, vous ne pourrez que très rarement répondre à une question à développements par une réponse simple et prémâchée. Normalement, on ne vous demandera pas de faire une liste, de décrire, de définir ou de faire un résumé de la matière. Et, même si vous êtes assez chanceux et qu'on vous le demande, il est fort possible que la personne qui a posé la question s'attende à ce que vous prouviez que vous pouvez comparer, mettre en contraste, évaluer, organiser selon la signification, avoir un esprit critique et résumer des éléments. Le seul fait que vous deviez répondre à une question à développements sous-entend, en effet, que vous devez faire tout ça.

À moins d'indications contraires, soyez prêt à faire preuve d'esprit critique chaque fois que vous avez à répondre à une question à développements. Si on vous demande de donner un aperçu, d'exposer les grandes lignes, d'illustrer, d'expliquer, de discuter, de critiquer ou d'évaluer, d'interpréter ou de réexaminer, on veut que vous fassiez une analyse critique (beaucoup plus de détails à ce sujet dans le prochain chapitre). La signification précise de chacun de ces mots est moins importante que ce qu'on attend de vous.

Il vaut tout de même la peine de réfléchir à ce qui distingue ces mots les uns des autres, puisqu'ils vous donnent des indications sur ce que veut votre professeur (ou votre employeur) :

- Donner un aperçu, c'est exposer l'évolution de quelque chose du début à la fin ;

- Exposer les grandes lignes, c'est vous concentrer sur les composantes principales de quelque chose;

- Illustrer, c'est fournir des exemples concrets pour étayer un argument ;

- Expliquer, c'est donner les raisons ou les causes de l'existence de quelque chose ;

- Débattre, c'est peser le pour et le contre de quelque chose ;

- Critiquer ou évaluer, c'est estimer en profondeur la valeur de quelque chose ;

- Interpréter, c'est trouver une signification profonde ou une tendance sous-jacente à quelque chose ;

- Réexaminer, c'est passer en revue un événement ou une explication pour l'analyser sous un autre angle.

Cependant, les différences subtiles entre ces mots ne sont rien comparativement au fait qu'on s'attendra à ce que vous fassiez une analyse critique qui décompose les différentes parties d'un problème pour en révéler les causes, les connexions et les corrélations. Si vous 
souhaitez exceller, vous devez non seulement faire la preuve que vous êtes capable de penser, mais aussi que vous pouvez le faire avec créativité et imagination.

Vos collègues et votre patron voudront aussi s'assurer que vous avez un esprit critique, que vous êtes créatif et que vous avez de l'imagination, peu importe les tâches que vous entreprenez. Les employeurs ne vous payeront certainement pas un gros salaire pour être ordinaire ou pour ressasser des lieux communs. Non : ils veulent des analyses en profondeur, des explications concises et des options vraisemblables, qui ajoutent de la valeur à ce que vous proposez. Exactement ce que veulent aussi vos professeurs!

Les examens à développements vous forcent à jongler avec des concepts, à élaborer vos propres théories sur la base d'idées existantes et à créer vos propres interprétations et vos propres synthèses. Mais, et c'est important de le souligner, ils vous obligent à faire la preuve que vous possédez et utilisez ces aptitudes complexes en respectant les échéances. Si on lui donne tout le temps du monde, n'importe qui peut réussir, mais le marché de l'emploi est très concurrentiel et rempli d'échéances. Ce n'est pas pour rien qu'en anglais, on appelle une échéance un deadline: votre carrière ne fera pas long feu si vous n'êtes pas capable de la respecter.

Vous trouverez ci-dessous une description des trois mots clés que vous rencontrerez le plus souvent quand vous ferez des examens à développements ou maison. Les trois mots - comparer, discuter et évaluer - sont importants, car ils dénotent le genre d'aptitudes critiques que les professeurs et les employeurs recherchent.

\begin{tabular}{|l|l|l|}
\hline Comparer & Discuter & Évaluer \\
\hline Quand vous comparez, & Pour étayer votre & Quand vous évaluez, \\
vous vous concentrez & discussion, abordez & vous passez au crible \\
sur les similitudes, les & directement les options & les raisonnements et \\
différences et les liens entre & possibles. Ne faites pas & les preuves à votre \\
les idées. Vos comparaisons & appel à un homme de & disposition, à l'aide de \\
doivent démontrer que & paille, c'est-à-dire à un & critères scientifiques, afin \\
vous comprenez bien les & argument que vous créez & de prendre position ensuite. \\
tenants et les aboutissants & dans le seul but de le & Vous devez également \\
des idées. & réfuter. & montrer à quel point \\
& & vous comprenez bien les \\
& problématiques. \\
\hline
\end{tabular}

Pour vous aider à répondre à des questions qui vous demandent de comparer, discuter ou évaluer, suivez ce guide ${ }^{1}$. 


\begin{tabular}{|l|l|l|}
\hline $\begin{array}{l}\text { Comparer: concentrez- } \\
\text { vous sur les similitudes, } \\
\text { mais prenez en note les } \\
\text { différences. }\end{array}$ & $\begin{array}{l}\text { Discuter: énoncez } \\
\text { clairement la thèse ou } \\
\text { l'hypothèse que vous avez } \\
\text { l'intention de prouver. }\end{array}$ & $\begin{array}{l}\text { Évaluer: faites le tour } \\
\text { des positions et des } \\
\text { discussions au sujet d'une } \\
\text { problématique donnée. }\end{array}$ \\
\hline $\begin{array}{l}\text { Opposer: concentrez- } \\
\text { vous sur les différences, } \\
\text { mais soyez conscient des } \\
\text { similitudes. }\end{array}$ & $\begin{array}{l}\text { Prouver: établissez la } \\
\text { validité de votre thèse à } \\
\text { l'aide de raisonnements } \\
\text { logiques et de preuves. }\end{array}$ & $\begin{array}{l}\text { Évaluer: indiquez } \\
\text { les avantages et les } \\
\text { inconvénients de chacune. }\end{array}$ \\
\hline $\begin{array}{l}\text { Faire des liens: montrez } \\
\text { comment les idées sont } \\
\text { liées entre elles. }\end{array}$ & $\begin{array}{l}\text { Débattre: mettez votre } \\
\text { thèse à l'épreuve en la } \\
\text { comparant avec d'autres } \\
\text { options. }\end{array}$ & $\begin{array}{l}\text { Interpréter: simplifiez } \\
\text { ce qui est complexe pour } \\
\text { mieux communiquer les } \\
\text { problématiques. }\end{array}$ \\
\hline $\begin{array}{l}\text { Évaluer: } \text { si vous comparez } \\
\text { des idées, vous devez } \\
\text { obligatoirement les évaluer. }\end{array}$ & $\begin{array}{l}\text { Justifier: montrez en quoi } \\
\text { votre thèse est la meilleure. }\end{array}$ & $\begin{array}{l}\text { Juger }: \text { déterminez la valeur, } \\
\text { l'exactitude et l'utilité } \\
\text { d'approches différentes. }\end{array}$ \\
\hline
\end{tabular}

Voyez-vous pourquoi quelqu'un qui peut comparer, discuter et évaluer sous pression et en respectant une échéance serrée est un atout pour une organisation? Si cette personne est également en mesure de porter des jugements exacts, ne serait-elle pas indispensable? Ajoutez-lui la capacité à convaincre les autres à l'aide d'arguments logiques et de données pertinentes pour appuyer chaque affirmation, et vous avez une perle rare!

\section{L'utilité des dissertations}

Être en mesure d'expliquer le pourquoi de quelque chose est essentiel à l'école comme au travail. Ce genre de question est en fait beaucoup plus fréquent que tout autre. Plus vous grimperez les échelons au travail, plus vos patrons, vos collègues, vos clients et autres parties prenantes s'attendront à ce que vous soyez en mesure d'expliquer pourquoi. Pourquoi mes actions ont-elles perdu de la valeur? Pourquoi avonsnous vu une augmentation de nos ventes ce trimestre? Pourquoi avons-nous moins de clients cette année? Pourquoi veut-il me voir? Pourquoi le projet n'est-il pas terminé ? Pourquoi ma fille ne réussitelle pas bien en deuxième année?

Évidemment, vous devrez également répondre à d'autres questions: quelles actions devrais-je acheter? Que devrais-je enseigner à mes élèves? Celles-ci exigeront aussi de vous que vous expliquiez pourquoi votre réponse est la meilleure.

Aussi, la compétence la plus importante que vous devez développer au cours de vos études postsecondaires est la capacité à expliquer 
pourquoi. Autrement dit, vous devez apprendre à convaincre les autres que votre interprétation d'un développement ou d'un événement précis est la bonne, et pas n'importe quels développements ou événements, mais des sujets complexes et difficiles à comprendre.

À un moment donné, au cours de vos études postsecondaires, vous devrez rédiger au moins une dissertation. On ne vous demandera probablement pas de faire de même au travail, ce qui pourrait vous inciter à croire que la rédaction de dissertations est une perte de temps ou une compétence difficilement transférable. Détrompezvous! Beaucoup d'employeurs exigent qu'une lettre de présentation accompagne le $\mathrm{CV}$ des candidats, car elle vise à expliquer pourquoi ils devraient vous embaucher. De plus, les entrevues comprennent souvent de courtes questions écrites. Les demandes d'admission aux études supérieures requièrent toutes des lettres d'intention qui répondent à la question : pourquoi devrions-nous vous admettre à ce programme?

Les dissertations vous permettent d'améliorer votre capacité à expliquer pourquoi et à développer considérablement votre aptitude à communiquer les résultats de vos recherches. Votre patronne ne vous demandera peut-être pas de rédiger une dissertation pour expliquer pourquoi les ventes ont diminué ce trimestre, mais elle voudra probablement un rapport qui explique pourquoi c'est arrivé, lequel émet des recommandations pour renverser la vapeur. Elle s'attendra de plus à ce que vous fassiez preuve de pensée critique et d'imagination, ce que vous aurez développé en rédigeant des dissertations. Nous explorerons plus en détail les sujets de la pensée critique et de l'imagination au chapitre suivant, ainsi qu'aux chapitres 7 et 8 .

La rédaction de dissertations vous apprend à expliquer des choses aux autres, peu importe qu'il s'agisse d'architecture byzantine ou d'investissements dans les produits de base. Les compétences interprétatives, critiques et analytiques qui entrent en jeu dans la rédaction d'une dissertation sont les mêmes, peu importe le sujet.

Vous êtes-vous déjà demandé comment font vos professeurs pour être aussi intelligents et habiles à expliquer des concepts compliqués? Eh bien, ils ont rédigé une tonne de dissertations, d'études de cas ou de rapports. Quand ils ont terminé leurs études collégiales ou de premier cycle universitaire, ils n'ont pas arrêté de rédiger des dissertations. Normalement, pour devenir professeurs, ils ont dû rédiger une thèse de doctorat, c'est-à-dire une très, très longue dissertation qui fait preuve d'originalité et d'aptitudes en recherche. Une fois embauchés 
en tant que professeurs, ils doivent rédiger des articles (de courtes dissertations) et des livres (de longues dissertations) afin de garder leur emploi et obtenir des promotions. Vous ne pensiez tout de même pas qu'ils passent leur temps à enseigner !

Vous ne planifiez probablement pas devenir professeur ou, en tout cas, pas pour l'instant, mais ça ne vous empêche pas de développer ces mêmes aptitudes critiques et créatives pour les utiliser dans votre carrière de prédilection. Profitez des dissertations pour vous exercer à expliquer les choses de façon concise, intéressante et fondée sur des faits. Il peut parfois être difficile de choisir un sujet de dissertation, en particulier si votre professeur ou son assistant d'enseignement vous laissent le faire. Vous pouvez vous en sortir en choisissant un sujet dont vous pourrez profiter après avoir obtenu votre diplôme. C'est comme ça que les dissertations et autres devoirs semblables vous donnent la chance d'explorer quel genre de carrière vous voulez. Servez-vous en pour devenir un expert dans un domaine précis.

Par exemple, pourquoi ne pas étudier les répercussions qu'un accord de libre-échange a sur le Canada et, plus particulièrement, sur les compagnies canadiennes ou les travailleurs canadiens? Par ailleurs, si un sujet est populaire dans les médias (comme une découverte, un débat ou une tragédie), pourquoi ne pas en étudier un aspect pour votre cours d'arts ou de sciences sociales? Quand vous commencerez à vous chercher un emploi, même si ce n'est que pour l'été ou à temps partiel, vous serez en mesure de prouver aux employeurs potentiels que vous avez une connaissance spécialisée et actuelle de ces sujets chauds.

Vous pouvez également vous pencher sur une tendance émergente. Par exemple, la population canadienne se fait vieillissante, les baby-boomers commençant à atteindre l'âge de 65 ans. Cette tendance touchera plusieurs aspects de la vie au Canada, du développement de produits aux politiques de soins de santé et aux régimes de retraite. $\mathrm{Si}$, au cours de votre carrière postsecondaire, vous rédigez plusieurs dissertations sur le vieillissement de la population, vous détiendrez un savoir qui sera prisé par les employeurs. Le plus beau dans tout ça, c'est que la plupart des organisations devront faire face à ce phénomène, des constructeurs automobiles aux banques, en passant par les agences de voyages.

Nike et Adidas devraient peut-être commencer à diversifier leurs produits et développer une gamme d'équipements de golf. Peu 
de personnes âgées continueront à courir sur de longues distances ; ça finit par être pénible pour les articulations vieillissantes! Les maisons d'édition feront une fortune quand de plus en plus de retraités auront le temps de lire, mais liront-ils en format imprimé, numérique ou autre? Quel genre de produits intéressent les consommateurs plus âgés?

Il est possible de choisir un sujet de dissertation ou de recherche pertinent pour vous et pour des employeurs potentiels dans n'importe quelle discipline, y compris en philosophie, en histoire et en anglais. Les scandales au sein du gouvernement, les fraudes commises par les compagnies, le taux de criminalité et les droits des individus soulèvent quelques-unes des questions clés étudiées par les philosophes.

Vous pouvez même apporter votre travail de recherche à l'entrevue. Référez-vous au chapitre 9 pour plus d'informations sur le processus d'entrevue.

Comme nous l'avons affirmé plus tôt dans ce livre, il est primordial de suivre des cours qui vous intéressent. Mais pourquoi ne pas aller un peu plus loin et choisir des sujets de dissertation qui vous allument? Soyez ensuite stratégique et faites des liens entre vos intérêts et le genre de sujets qui peuvent intéresser les employeurs ou fournir une base solide à votre future carrière. La rédaction de bonnes dissertations demande beaucoup de travail, mais elle est agréable si le sujet vous intéresse.

Vous pouvez transformer vos intérêts personnels en stratégie de carrière. Dès que vous avez déterminé un champ d'intérêt, explorezle en suivant une grande variété de cours qui s'y rapportent. Avec le temps, vous en connaîtrez plus que tout le monde sur le sujet, ce qui fera de vous un expert en la matière et, en fin de compte, vous rendra plus attrayant sur le marché du travail.

Il ne suffit pas de simplement vous intéresser à un sujet particulier. Vous devez être rigoureux dans votre approche. La rédaction postsecondaire, plus précisément la rédaction scientifique, est la prose la plus objective qui soit. Celui qui s'adresse à un lectorat d'érudits dans un domaine donné doit obligatoirement communiquer l'information la plus précise, la plus objective et la plus exacte qui soit. Les hyperboles et les formulations trop métaphoriques n'ont pas leur place en rédaction de niveau postsecondaire, car elles diminuent l'efficacité du langage simple et entravent la communication claire.

De la même manière, la communication claire est absolument essentielle dans le monde du travail. La rédaction professionnelle ou 
d'affaires doit être objective pour être efficace. Un rapport dramatique ou au langage fleuri, partial ou inexact, mènera à de mauvaises décisions. Toute décision devrait être prise sur la base de faits, de preuves, de logique et de pondération. Vous devez donc rédiger de cette façon pour réussir dans le monde du travail. Il n'y a pas de meilleur moyen d'apprendre comment faire qu'en rédigeant des travaux, des rapports ou des études de cas pendant vos études.

Les spécialistes de la santé et du droit, les travailleurs sociaux, les policiers, les infirmiers, les enseignants, les journalistes et beaucoup d'autres professionnels doivent être objectifs et impartiaux. Quand ils rédigent ou parlent, ils le font après avoir soigneusement évalué et analysé les faits, et considéré une hypothèse plausible pour les expliquer. Cela ne signifie pas qu'ils ne peuvent pas avoir d'opinion. Cela veut dire qu'ils doivent exprimer clairement cette opinion, l'étayer à l'aide de théories et de faits, et justifier pourquoi ils la préfèrent à d'autres.

Les étudiants se plaignent parfois du fait que les professeurs sont «trop difficiles » ou qu'ils « auraient dû savoir» de quoi il était question dans la dissertation. Cette attitude, qui se manifeste souvent à la suite de la réception de notes, n'a pas sa place dans un environnement postsecondaire, où la rédaction doit être précise et claire. Les fautes grammaticales et stylistiques ainsi qu'un raisonnement erroné sont pénalisés, car une rédaction faible porte ombrage au sens et entrave la communication.

Vos professeurs remettront en question les raisonnements qui ne s'appuient pas sur des faits, non pas parce qu'ils ne sont pas d'accord avec vous, mais parce que cette façon d'écrire trahit un manque d'érudition. Dans le monde professionnel, on s'attendra à ce que vous appuyiez toute affirmation que vous ferez. Si vous avez tendance à faire des affirmations à l'emporte-pièce, sans réfléchir ou sur la base de vos émotions ou de votre intuition, vos employeurs et vos collègues vous apposeront l'étiquette d'impulsif. Le problème avec les impulsifs, c'est qu'ils manquent souvent d'exactitude et peuvent même menacer le bon fonctionnement du bureau. Ils sont donc plus sujets aux renvois ou aux rétrogradations dans des postes où ils ne peuvent pas causer trop de dommages.

Un professeur fera probablement preuve d'une certaine souplesse à votre égard si vous vous empêtrez dans votre raisonnement, si vous ne fournissez pas assez de faits pour soutenir vos arguments ou si la qualité de votre prose laisse à désirer. Les études postsecondaires 
sont, après tout, l'occasion d'apprendre et apprendre signifie nécessairement faire des erreurs. Par contre, soyez assuré que la personne qui paie votre salaire et compte sur votre expertise de manière très concrète ne vous donnera aucune chance.

\section{Écrire pour un lectorat d'érudits}

Peu importe ce que vous rédigez ou quand vous le rédigez, n’oubliez jamais pour qui vous le faites. Beaucoup d'étudiants tentent de rédiger pour un professeur précis et perdent leur temps à essayer de trouver ce qui lui plaira. Certains vont même plus loin et font semblant d'être d'accord avec les idées ou les théories du professeur.

Cette approche est très peu judicieuse. Elle fonctionnera peutêtre avec un ou deux professeurs, mais elle vous rapportera très peu. Il est plus intelligent de dépenser votre énergie à construire un raisonnement solide. Au moins, de cette façon, vous développerez, dans un cadre postsecondaire, les compétences qui feront de vous un meilleur rédacteur.

Chaque année, les auteurs de ce livre se voient obligés de donner de mauvaises notes aux étudiants qui répètent bêtement ce qu'ils croient que nous voulons entendre, au lieu de faire leurs propres recherches et développer leur propre opinion. Il est difficile de respecter ceux qui s'engagent dans la voie de la flatterie et qui, de toute évidence, ne veulent pas faire les efforts nécessaires pour apprendre.

La même chose s'applique au monde du travail. La plupart des patrons n'aiment pas ceux qui sont toujours d'accord avec eux. Certes, vous pouvez rédiger des rapports et des notes de service qui plaisent au patron, mais ça ne vous servira pas longtemps. Dès que vous vous serez fait une réputation de lèche-botte, votre patron ne vous respectera plus et vos collègues ne vous feront plus confiance. Votre carrière sera compromise et vous pourriez même en venir à ne plus vous respecter vous-même. Il est de loin préférable de rédiger de manière professionnelle et qui convient à l'environnement dans lequel vous travaillez.

Toute dissertation postsecondaire doit posséder les sept qualités suivantes ${ }^{2}$ :

1. Intégralité et exhaustivité. Vous devez situer clairement votre travail à l'intérieur d'un ensemble de connaissances, démontrer comment il y contribue, en commençant par un énoncé du problème, pour ensuite procéder logiquement jusqu'à sa résolution. 
Dans le milieu du travail, on dit souvent : «ne réinventez pas la roue». Quand vous rédigez, construisez votre raisonnement à partir de connaissances qui existent déjà ;

2. Exactitude. Vous devez fournir de l'information exacte. Cela signifie que vous devez vérifier toutes vos affirmations pour vous assurer qu'elles n'embrouillent pas les données ou ne mènent pas à de fausses conclusions. L'exactitude en rédaction de dissertations exige des étudiants qu'ils pèsent bien leurs mots et qu'ils soient prêts à défendre toutes les affirmations qui ne sont pas vérifiables.

Si vous traficotez les données, vos employeurs et vos gestionnaires seront particulièrement sévères envers vous, car cela pourrait mener à de mauvaises décisions et à des situations pour le moins embarrassantes ;

3. Objectivité. Toute dissertation doit être objective. Les auteurs de ces travaux scolaires, qu'ils soient étudiants ou professeurs, établissent habituellement d'emblée leur cadre théorique ou leurs partis pris, afin de permettre aux lecteurs d'évaluer eux-mêmes la présentation des données. Ils informent les lecteurs des limites de leur travail et font état des interprétations qu'ils ont rejetées. Ils n'exagèrent pas l'importance des résultats de leurs recherches. Par-dessus tout, ils ne cachent pas les données gênantes ou qui ne leur conviennent pas.

De la même façon, votre employeur ou votre gestionnaire, vos collègues et vos clients comptent sur le fait qu'ils reçoivent de l'information objective. Au moment de décider de la marche à suivre, ils prennent habituellement plusieurs facteurs en ligne de compte, y compris les autres options. Toutefois, si leur décision se fonde sur de l'information partiale, le résultat est voué à l'échec dès le départ ;

4. Ordre. Les rédacteurs inexpérimentés ont souvent de la difficulté à organiser leurs idées sur papier. La rédaction scolaire au niveau postsecondaire expose le matériel selon un ordre logique. Les données complexes ou problématiques sont soigneusement présentées et l'information superflue est omise pour ne pas nuire à la lecture et au développement du sujet. Chaque section de la dissertation doit être clairement structurée et les transitions entre les sections doivent démontrer que le sujet se développe logiquement. Les idées 
et l'information comprises dans chaque paragraphe doivent être liées entre elles.

La rédaction scolaire de niveau postsecondaire suit généralement un ordre chronologique pour éviter de ballotter sans cesse le lecteur du présent au passé, du présent au futur, et du futur au passé. Comme nous le ferons remarquer plus loin dans ce chapitre, réviser son texte est essentiel pour aboutir à un produit final logique et ordonné.

L'ordre et le développement logique sont essentiels dans le monde des affaires et celui du gouvernement. Habituellement, les documents commencent par un résumé, qui condense le raisonnement et met en évidence la manière dont l'ensemble se tient. Si des liens sont ténus ou manquants, le rapport entier sera rejeté. Pendant vos études, on ne vous demandera peut-être pas de rédiger des résumés, mais vous ne perdez rien à les inclure à vos devoirs écrits. Non seulement développerez-vous ainsi votre aptitude à résumer de longs documents en quelques centaines de mots, mais vous impressionnerez peut-être votre professeur ou l'assistant à l'enseignement, et obtiendrez une meilleure note ;

5. Pertinence. Pourquoi les étudiants ont-ils du mal à mettre en ordre leur matériel ? Parce qu'ils n'organisent pas clairement les résultats de leur recherche ou parce qu'ils souhaitent tellement montrer à quel point ils ont travaillé fort et combien ils en savent sur le sujet, qu'ils entassent tout ce qu'ils ont dans une dissertation, même si ce n'est pas pertinent.

Dans le passé, on a peut-être récompensé votre ténacité et votre capacité à retenir l'information. Dans le monde postsecondaire, on s'attend toujours à ce que vous travailliez, mais on récompense la clarté et la pertinence. C'est pourquoi il est possible que vous obteniez une note beaucoup plus basse que quelqu'un qui a travaillé beaucoup moins fort et qui en connaît moins sur le sujet.

C'est simple : les employeurs ne tolèrent pas les raisonnements qui manquent de pertinence ou qui font fausse route. Ils paient leurs employés pour qu'ils se concentrent sur les problèmes à régler et toute digression leur fait perdre temps et argent.

Voici un dicton du monde des affaires qui pourrait vous être utile: ne vous contentez pas de travailler, travaillez intelligemment ; 
6. Simplicité. En rédaction de niveau postsecondaire, vous devez démontrer que vous maîtrisez le matériel et que vous pouvez l'expliquer efficacement. Vos professeurs vous demanderont de vous justifier si vos mots et vos idées ne sont pas simples et faciles à comprendre. Traduire en langage simple des idées complexes et subtiles est la meilleure façon de prouver que vous les comprenez.

Les auteurs de ce livre ont rédigé beaucoup de documents pour des organisations des secteurs public et privé, tant sur le plan national que sur le plan international. Notre but était toujours d'écrire le plus simplement possible et nous avons dû réviser nos textes plusieurs fois avant d'atteindre cette simplicité ;

7. Clarté. Si vous suivez les six étapes précédentes, vous produirez un texte clair et vous simplifierez ce qui est complexe. Pour clarifier, vous devez être en mesure de distinguer entre les arguments logiques et les attraits émotifs. Clarifier exige de vous débarrasser de ce qui s'éloigne du sujet pour pouvoir communiquer avec précision. La clarté découle d'un langage précis et élégant, non pas d'une prose alambiquée et imagée. Enfin, écrire clairement signifie expliquer quelque chose efficacement dès le départ au lieu de le répéter inutilement.

Si vous relisez les sept points mentionnés plus haut, vous verrez que chacun d'eux peut s'appliquer à n'importe quelle forme de rédaction. Comment, en effet, pourrait-on vous prendre au sérieux si votre présentation, votre note de service, votre mémoire juridique ou votre rapport de cas pour la garde d'enfant n'est pas exact, exhaustif, pertinent, objectif et concis? Croyez-vous vraiment que vos collègues, vos gestionnaires, vos clients et tous les autres prendront vos idées, vos commentaires et vos suggestions au sérieux si vous ne respectez pas les indications ci-dessus?

Dans un milieu où le temps, c'est de l'argent, et où vos gestionnaires doivent prendre des décisions sur la base d'informations exactes, vous aurez besoin des compétences qui s'acquièrent en rédigeant de bonnes dissertations. Ce n'est pas par hasard que les meilleurs rédacteurs pendant les études finissent par obtenir les carrières les plus prometteuses après celles-ci. 


\section{Comment organiser une dissertation}

Une dissertation ne s'organise pas de la même manière que votre recherche, laquelle est structurée par thèmes et sous-thèmes. Évidemment, ces derniers participent à l'apport critique et au raisonnement de votre dissertation. La substance de celle-ci provient de votre recherche (voir le chapitre 6 pour plus de détails). Mais les bonnes dissertations de niveau postsecondaire sont organisées selon des conventions de structure auxquelles les lecteurs s'attendent.

Quand vous ouvrez un roman, vous vous attendez à ce qu'il ait une structure particulière. Les poèmes prennent aussi différentes formes, selon qu'ils sont sonnets, élégies ou épopées. Les pièces de théâtre ont généralement un nombre précis d'actes, lequel peut varier selon les normes culturelles de l'époque où elles ont été écrites. De la même manière, les dissertations scolaires s'inscrivent dans au moins une des structures suivantes.

\section{Structure argumentative}

Beaucoup d'essais scientifiques et d'articles prennent la forme de discussions ou de débats. Une dissertation argumentative doit développer une thèse claire - un argument - qui est appuyée par des preuves. Elle commence généralement par l'énoncé de la thèse. Puis, elle décrit un ou plusieurs arguments qui la contredisent. Après l'évaluation de ces arguments, elle démontre pourquoi la thèse avancée est la meilleure.

Le lecteur (votre professeur) voudra savoir si vous comprenez vraiment les forces et les faiblesses des approches que vous rejetez.

\section{Structure explicative}

Une dissertation explicative utilise des méthodes sophistiquées pour faire la lumière sur le sujet dont il est question. Ces méthodes peuvent être des approches disciplinaires, comme le modernisme ou le postmodernisme, ou des modèles théoriques, comme le freudisme ou le marxisme. En les appliquant, la dissertation interprète ou explique le matériel. Ce genre de dissertation permet d'aller bien au-delà de l'intuition et du gros bon sens pour découvrir les tendances et les significations profondes du sujet, et constitue donc un des outils les plus précieux des étudiants. 
Votre lecteur cherchera à déterminer si vous comprenez les cadres théoriques et si vous les utilisez correctement. Beaucoup d'étudiants tentent d'utiliser des théories sans les comprendre, ce qui donne de bien piètres résultats.

\section{Structure descriptive}

Une dissertation descriptive évite d'interpréter le matériel ou de défendre une thèse. Elle vise plutôt à décrire un processus ou à transmettre de l'information factuelle qui sera utile au lecteur. Elle requiert une sélection soignée des faits pertinents ainsi qu'une capacité à communiquer avec clarté et concision.

Le lecteur se demandera : «que manque-t-il au tableau? » Vous ne pourrez pas, en effet, tout inclure dans votre dissertation descriptive, mais assurez-vous de ne rien oublier d'important.

\section{Structure comparative/exploratoire}

Une dissertation comparative aura deux approches différentes, lesquelles seront appliquées à un problème ou à une question. Il peut s'agir de cadres théoriques, comme le freudisme ou le marxisme, ou de deux points de vue sur un sujet controversé, comme l'avortement ou la discrimination positive. Les deux approches sont évaluées et l'une est généralement préférée à l'autre.

Le lecteur voudra savoir si vous avez compris les forces et les faiblesses des deux approches, si vous avez réussi à les comparer et si vous avez trouvé de bonnes raisons de rejeter l'une d'elles. N'essayez pas d'obtenir des points en mettant de l'avant des arguments faibles simplement parce qu'il vous sera facile de les réfuter. Un lecteur perspicace verra tout de suite qu'ils ne valent rien.

\section{Structure progressive}

Une dissertation progressive se basera sur des arguments en apparence insignifiants pour arriver à une conclusion solide. Chaque argument prend appui sur le précédent pour créer une construction impressionnante.

Le lecteur tentera de voir si vous avez sauté des étapes dans votre construction. 


\section{Structure synthétique}

Une dissertation synthétique utilisera les éléments les plus appropriés et pertinents des différents types de dissertations énumérés afin de créer le meilleur raisonnement possible. Les bons raisonnements sont complexes et subtils, et se fondent rarement sur une seule approche.

Le lecteur, qui sait à quel point il est difficile d'en arriver à un raisonnement synthétique, voudra vérifier si vous avez réussi à appliquer les théories et les approches pertinentes pour offrir une bonne perspective sur le matériel.

Si vous relisez les types de dissertations, vous verrez que chacun possède un équivalent dans le milieu professionnel. À un moment ou à un autre de votre carrière, vous devrez probablement rédiger un rapport à la suite de l'analyse de deux stratégies d'affaires différentes et vous prendrez position en faveur de l'une d'elles ou vous devrez expliquer l'échec d'un projet précis, ou encore on vous demandera de simplement décrire les faits tels qu'ils sont.

Les bons rapports ou les bonnes études de cas d'affaires comportent souvent des éléments provenant de chacun des types de dissertations. Ils commenceront peut-être par une description détaillée et claire de la situation ou du problème. Ils présenteront peut-être l'information progressivement et logiquement pour arriver à discerner des tendances. Ils pourront utiliser certaines théories et interprétations pour offrir des options. Ils amalgameront peut-être plusieurs approches et théories pour arriver à une solution, une recommandation ou une interprétation unique et créative.

\section{La structure de base de la dissertation}

La structure de base d'une dissertation ou d'un rapport professionnel est très simple. Elle comprend un début, un milieu et une fin. Le début présente le sujet. Le milieu développe les différentes étapes de votre raisonnement et prouve les affirmations que vous avez faites dans votre introduction. La conclusion résume ce que vous avez affirmé, prouvé et découvert. Elle peut également inclure les conséquences futures que pourraient avoir les résultats de vos recherches. 


\section{L'introduction}

Le plus difficile, c'est de commencer, mais ne vous compliquez pas la vie pour autant. Vous n'avez pas besoin d'une ouverture dynamique pour vous mettre en marche. Vous devez simplement présenter votre sujet - le problème, la question ou le dilemme -, faire suivre cette présentation d'une mise en contexte, et dire au lecteur ce que vous avez l'intention de prouver ou de démontrer. En d'autres termes, cette section de la dissertation énonce le problème, la controverse, le débat ou la question qui sera traité dans la dissertation.

Même si elle est courte, cette section doit mettre en évidence l'importance de ce qui suivra. Selon la complexité de votre sujet, l'introduction peut tenir en un seul paragraphe ou s'étendre sur quelques pages. Il n'existe pas de règles absolues quant à sa longueur.

On a probablement répété à plusieurs d'entre vous qu'il est important de commencer une dissertation par la thèse. Beaucoup de guides de rédaction insistent, en effet, sur le fait que la thèse doit énoncer clairement ce que la dissertation tente de prouver.

Quoique bien intentionné, ce conseil est en fait n'importe quoi. Quelques-unes des meilleures dissertations et certains des rapports les plus percutants jamais écrits ne suivent pas cette règle.

Beaucoup de professeurs souhaiteront que votre dissertation commence par l'énoncé de la thèse. Si c'est votre cas, assurez-vous de le faire. Sinon, il importe d'énoncer, tôt dans votre introduction, une idée directrice qui guide votre raisonnement ou vers laquelle vous vous dirigez clairement. Il n'est pas nécessaire que ce soit un énoncé de thèse. Vous pouvez simplement présenter la question à laquelle vous tenterez de répondre, le problème auquel vous trouverez une solution ou le dilemme que vous explorerez.

Il est fréquent de voir les étudiants fournir la réponse à la question, au problème ou au dilemme dans leur introduction. Pourriezvous concevoir un roman policier dont la première phrase révélerait que «le meurtrier est le majordome»? L'affirmation qui guide un roman policier, la plupart du temps sous-entendue plutôt qu'énoncée, est «Qui a commis le meurtre? » Cette affirmation sert également de cadre et de principe organisateur à l'ensemble du livre.

Il n'y a pas de mal à garder un peu de suspense pour votre lecteur dans l'introduction. Vous devez expliquer le sujet de votre dissertation, certes, mais pas nécessairement tout dévoiler d'un coup. Par exemple, si votre texte porte sur les répercussions du vieillissement de 
la population sur le design des voitures, vous n'en ferez probablement pas tout de suite la liste. Présentez plutôt le sujet comme potentiellement important et intéressant. Essayez d'accrocher le lecteur. Une fois accroché, il voudra savoir comment le vieillissement de la population aura des répercussions sur le design des voitures, tel que vous le démontrerez par les preuves que vous aurez découvertes pendant vos recherches.

Une erreur souvent commise par les étudiants inexpérimentés est d'énoncer une thèse avant d'avoir fait des recherches sur leur sujet et, ensuite, de forcer les résultats de leur recherche à appuyer la thèse. Procuste, un brigand de la mythologie grecque, faisait quelque chose de similaire en forçant les voyageurs à se coucher dans un lit de fer. Si ses victimes étaient trop petites, il les étirait; si elles étaient trop grandes, il leur coupait les jambes. Ne vous faites pas un lit de Procuste!

L'introduction se termine en donnant un aperçu du reste de la dissertation. Dites quelque chose comme: "La première section de cette dissertation fera... La seconde section... ». Vous éviterez ainsi au lecteur d'avoir l'impression de faire un tour de montagnes russes les yeux bandés. Dites-lui brièvement à quoi il doit s'attendre. L'utilisation de sous-titres dans le corps de votre texte aidera parfois à l'orienter.

\section{Le développement}

Il vous est littéralement possible de déterminer combien de paragraphes votre dissertation contiendra. Vous n'avez qu'à additionner les arguments principaux de votre raisonnement et les concepts que vous devez développer. Selon la règle de base habituelle, vous aurez besoin d'au moins un paragraphe pour chaque concept. Ces composantes sont essentielles à la structure de votre dissertation. Si votre texte porte sur l'économie, par exemple, vous pourriez discuter des concepts d'intérêt personnel, de concurrence, de propriété privée et de profits. Nous développerons davantage les concepts dans le prochain chapitre. Pour l'instant, nous nous concentrerons sur leur organisation.

Si vous avez fait vos recherches correctement, vous devriez déjà avoir organisé vos concepts dans un fichier, dans votre tête ou sur papier. Ce ne serait peut-être pas non plus une mauvaise idée d'écrire vos concepts sur des papiers autocollants (Post-it) ou sur des fiches pour avoir la liberté d'en modifier l'ordre quand vous commencerez 
à rédiger votre dissertation. Peu importe à quel point vous avez organisé vos recherches, le processus d'écriture vous forcera à réorganiser vos idées à un moment ou à un autre. Les papiers autocollants vous permettront de déplacer les concepts et d'éviter de rester coincé dans votre raisonnement.

La structure et la taille de chaque paragraphe sont déterminées par la quantité de texte que vous devez produire pour développer chaque concept. Tant que vous couvrez l'essentiel, il importe peu que les paragraphes soient courts ou longs. Il vous arrivera peut-être d'avoir très peu à dire sur certains concepts. Pour les étoffer, vous devrez peut-être faire un peu plus de recherches.

Les concepts sont liés les uns aux autres pour former un raisonnement. Le premier indicateur vous permettant de décider si votre information et vos concepts sont assez développés est la séquence logique de votre raisonnement. Au fur et à mesure que vous composez le corps de votre dissertation, relisez-vous constamment pour vous assurer que vos paragraphes suivent une progression logique.

Vous saurez si votre texte se lit bien en vous posant les questions suivantes :

- Puis-je avancer et reculer logiquement à partir de n'importe quel point de mon raisonnement?

- Puis-je rapidement faire un diagramme de ma dissertation sur un tableau ou un morceau de papier?

- Puis-je ajouter de la subtilité ou de la complexité sans compliquer la lecture?

\section{La conclusion}

Même si l'assistant à l'enseignement ou votre professeur lira votre texte en entier, la conclusion doit constituer une suite logique et claire de l'introduction. Vous saurez que ces sections sont à la fois solides et complètes si vous pouvez les lire, sans lire le reste, et comprendre l'essence du texte. De plus, si certains concepts et arguments dans le corps de votre dissertation sont plutôt obscurs ou déroutants, une introduction et une conclusion solides permettront à votre lecteur de s'y retrouver.

Parfois, dans le monde des affaires, seules l'introduction et la conclusion d'un document professionnel seront lues avec attention. Les cadres supérieurs, toujours très occupés, liront souvent le reste en diagonale. C'est pourquoi il est judicieux d'être extrêmement clair, concis et précis dans vos introductions et vos conclusions. 
Une conclusion médiocre à la fin d'une dissertation qui est, autrement, intelligente et bien composée constitue une véritable tragédie. Beaucoup d'étudiants sabotent en effet leurs efforts :

- en ne rédigeant pas de conclusion qui se respecte («Enfin, c'est terminé !») ;

- en introduisant de nouvelles idées ou du nouveau matériel («Oh, en passant...»);

- en étant indécis ou en manquant de fermeté («Je ne sais plus où $\mathrm{j}^{\prime}$ en suis »).

Il peut être possible de comprendre la première offense. Quand vous aurez développé tous vos arguments, vous aurez probablement l'impression que vous avez fait ce que vous aviez à faire. Faux! Vous devez résumer ce que vous avez prouvé ou découvert ou, à tout le moins, faire ressortir ce que cela signifie. Il n'y a rien de plus frustrant qu'une dissertation qui crée de l'anticipation et qui n'aboutit jamais. C'est l'équivalent scolaire des gens qui ne finissent pas leurs phrases et présument que vous savez ce qu'ils veulent dire.

La seconde offense, l'introduction de nouvelles idées ou d'informations, est inexcusable. Vous serez peut-être tenté d'en ajouter, mais la conclusion n'est pas l'endroit pour le faire. Même s'il vous est permis d'y suggérer des pistes pour des recherche futures, votre conclusion ne devrait jamais contenir de nouveau matériel.

Enfreindre cette règle distrait votre lecteur du raisonnement que vous venez de mettre des pages à prouver. Pire encore, il pourrait se demander quels autres éléments n'ont pas été considérés dans votre dissertation.

Habituellement, les étudiants (et les rédacteurs en général) ajoutent de nouvelles informations et d'autres idées parce qu'ils les ont découvertes au moment de terminer leur dissertation. Le secret (de Polichinelle) pour éviter qu'une telle situation ne se produise est de ne pas rédiger votre travail à la dernière minute. Ainsi, si vous avez de nouvelles idées ou si vous découvrez de nouveaux faits intéressants qui sont trop importants pour que vous les ignoriez, vous aurez le temps de les intégrer à votre dissertation.

Une autre façon d'éviter d'ajouter de nouvelles idées dans votre conclusion est de choisir un sujet, une question, un problème ou un dilemme très précis et limité. Si votre dissertation est guidée par une affirmation générale, vague ou très englobante, vous disposerez naturellement de plus d'informations que vous ne pourrez en inclure dans la discussion. 
Vous pouvez simplement affirmer, dans les premières pages de votre dissertation, que des événements en Chine, par exemple, ont été déterminants dans le déclenchement de la guerre du Vietnam, mais que vous ne vous y attarderez pas, par manque de place. Il est de loin préférable d'adopter cette approche que d'attendre le dernier paragraphe pour affirmer que des développements en Chine ont été déterminants dans le déclenchement de la guerre du Vietnam. En règle générale, évitez d'utiliser «aussi » et «de plus » dans les deux dernières pages de votre dissertation.

La troisième offense, être indécis ou vague, est parfois difficile à éviter. Il est normal de vouloir jouer de prudence au cas où vous auriez oublié quelque chose d'essentiel, ou votre dissertation ne serait pas parfaite, ou parce que vous ne faites pas confiance à vos arguments ou à vos résultats. Il n'en reste pas moins que votre lecteur, qui a suivi votre raisonnement du début à la fin, sentira que vous l'avez laissé tomber.

Vous saurez que votre conclusion est efficace si vous avez un sentiment d'accomplissement quand vous la révisez. Vous et votre lecteur devriez avoir une impression de déjà-vu. Ne laissez rien sans explication.

Sachez que le monde des affaires ne tolère pas l'indécision. Si vous composez une étude de cas pour votre patron, vous prenez position. Les gens d'affaires doivent prendre des décisions difficiles en respectant des échéances strictes; ils ne peuvent pas se permettre d'être indécis. Commencez donc tout de suite à prendre position!

\section{La rédaction de dissertations est un processus}

La rédaction de dissertations et de rapports prend du temps. C'est un processus, pas un événement mystérieux qui arrive la veille de la date limite. Comme nous le verrons plus loin, ce processus implique la production de plus d'une ébauche, le polissage de la version finale, la citation des sources et le choix d'un titre approprié.

\section{La première ébauche}

Vous ferez le plus dur du travail lors de la création de la première ébauche de votre dissertation ou de votre rapport. C'est là que vous amalgamerez réflexion, recherche et rédaction pour construire un raisonnement qui : 
- énonce clairement une thèse, un problème ou un dilemme ;

- aborde et règle un problème valable;

- démontre un usage efficace de preuves;

- est à l'image de votre propre point de vue ;

- établit un ou plusieurs points mémorables (pour vous et votre lecteur).

Atteindre tous ces buts n'est pas facile, même pour les rédacteurs d'expérience. À un moment donné, lors de la rédaction de votre ébauche, vous frapperez un mur. Quand cela se produira, essayez l'une des techniques suivantes:

- passez à une autre partie de votre dissertation, là où vous serez peut-être plus inspiré ;

- prenez une pause, relaxez et laissez les idées venir à vous sans les forcer;

- rédigez la page de titre et la bibliographie ;

- rédigez ce que vous savez et ne vous en faites pas au sujet de ce que vous ne savez pas ;

- discutez de la section problématique avec votre professeur ou un autre étudiant;

- faites plus de recherches.

Quand vous êtes bloqué, le mieux à faire est de relaxer sans complètement arrêter de penser à votre dissertation. Tant et aussi longtemps qu'une petite partie de votre esprit reste connectée à votre travail, votre subconscient réfléchira au problème et la solution se présentera souvent d'elle-même. Si vous forcez trop votre esprit, votre subconscient ne peut pas régler le problème pour vous. Inversement, si vous laissez complètement aller le problème, il pourrait avoir grossi quand vous y reviendrez.

Transporter un carnet de notes (papier ou électronique), dans lequel vous pouvez rapidement noter vos idées quand vous en avez, est une excellente habitude à prendre. Les rédacteurs professionnels expérimentés le font systématiquement. Plusieurs ont même un bloc-notes et un crayon, une tablette ou un téléphone intelligent sur leur table de nuit pour prendre en note toute révélation qui surgit au milieu de la nuit. Il n'y a rien de plus frustrant que de se rendormir et d'avoir complètement oublié notre brillante idée, le matin venu! 


\section{La deuxième ébauche}

On a déjà demandé à une écrivaine de renom de révéler le secret de son succès. Sa réponse fut instantanée et simple : réécrire. Si vous deviez appliquer le principe d'une analyse du rapport coûts-avantages à la rédaction de dissertations, vous verriez que consacrer une journée à la révision est ce qu'il y a de plus rentable. Voici quelques trucs pour guider votre réécriture :

- Attendez toujours quelques jours avant de rédiger votre deuxième ébauche. Si vous avez encore le sujet à l'esprit, vous ne serez pas en mesure de repérer les problèmes de manière objective ;

- Abordez ce que vous avez rédigé comme si c'était le produit d'un camarade de classe, et non pas le vôtre ;

- Vérifiez les transitions entre les paragraphes. Sont-elles fluides et utiles au lecteur?

- Lisez votre dissertation à voix haute pour déterminer si elle a du sens et coule bien.

Lire votre travail à voix haute est une stratégie de communication extrêmement efficace. Voici ce que vous pourriez découvrir ce faisant au sujet de votre travail :

- Son effet d'ensemble, son ton et son rythme;

- Si les idées sont claires ou éparpillées ;

- S'il comporte des écarts ;

- Les arguments qui fonctionnent et ceux qui ne fonctionnent pas;

- S'il y a de la répétition.

Cette stratégie sera encore plus efficace si vous vous enregistrez et vous vous écoutez ensuite.

À cette étape, ne vous concentrez pas sur l'orthographe et les règles de grammaire. Si vous révisez mot à mot, vous ne pouvez pas résoudre des problèmes de manière créative. De plus, vous perdez votre capacité à vous mettre à la place d'un lecteur intéressé à votre dissertation.

\section{L'ébauche finale}

À l'étape de l'ébauche finale, vous procéderez à la révision en profondeur dont a besoin toute dissertation. C'est le moment de vérifier la grammaire, la ponctuation, l'orthographe et le choix des mots. De 
nos jours, les correcteurs d'orthographe intégrés aux logiciels de traitement de texte facilitent le processus de révision, mais ils ne peuvent pas tout corriger et induisent parfois en erreur. La révision attentive de votre travail pourrait faire la différence entre un $\mathrm{B}+$ et un $\mathrm{A}$.

Les étudiants se demandent souvent pourquoi ils sont pénalisés pour des erreurs qui n'ont rien à voir avec leur capacité à réfléchir. Voici quatre raisons :

- Les fautes d'orthographe et les coquilles indiquent un manque de rigueur et d'attention, et de la précipitation ;

- Un vocabulaire limité vous empêche de dire ce que vous voulez vraiment dire ;

- Une mauvaise ponctuation rend difficile la lecture de votre dissertation et force le lecteur à relire les mêmes phrases ;

- Une grammaire pauvre amoindrit l'effet d'une phrase ou d'un paragraphe et en change ou en embrouille souvent le sens.

Les organisations et les compagnies tolèrent très mal les erreurs de grammaire dans leurs documents. Vos supérieurs ne sont pas payés pour trouver et corriger vos erreurs. Un zéro de trop dans un document peut avoir des conséquences légales, financières et personnelles pour vous et votre employeur.

\section{La révision}

Il est très difficile, et parfois impossible, de réviser votre propre texte. Vous aurez du mal à trouver les problèmes dans ce que vous avez vous-même écrit, qu'il s'agisse de grammaire ou de contenu. Aucun auteur n'est en mesure de réviser son propre travail de façon experte.

Quand vient le moment de la révision, faites appel à vos collègues. Dans le monde professionnel, il s'agirait de vos collègues ou d'un réviseur professionnel. Pour une dissertation, essayez de réunir deux sortes de réviseurs: les premiers liront les premières ébauches et vous donneront des conseils s'il y a des problèmes de logique, de structure, de raisonnement, etc. Les seconds liront l'ébauche finale et s'attarderont seulement aux problèmes de rédaction.

Ne faites pas l'erreur de demander à votre famille et à vos amis de relire votre travail. Ceux-ci voudront naturellement vous encourager et auront probablement tendance à vous dire: "Chéri, c'est la meilleure chose que tu as écrite!» De tels commentaires ne vous 
aideront aucunement. Trouvez des collègues qui sont critiques, qui vous questionneront et feront ressortir les failles, les problèmes, les incohérences et toute autre faiblesse. Quand vous trouverez de telles personnes ou quand vous les aurez formées, traitez-les bien, car leur apport vous permettra d'améliorer considérablement les notes que vous recevrez pour vos dissertations.

La meilleure façon de conserver vos réviseurs est de leur rendre la pareille. Ils porteront autant d'attention à votre travail que vous en porterez au leur. Si vous n'avez pas leur talent pour rédiger ou pour réviser, rendez-leur service d'une autre façon.

Beaucoup de sociétés de premier ordre ont mis en place des stratégies collaboratives pour réviser les lettres et les documents importants, lesquels seront lus et révisés, avec commentaires, par plusieurs personnes à chaque étape du processus de rédaction. On attend de chacun qu'il prenne ses responsabilités de réviseur très au sérieux car le groupe en entier se trouve dans le pétrin si quelqu'un manque à la tâche. Tout document gouvernemental est révisé par au moins six personnes avant d'être publié !

\section{La citation des sources}

Beaucoup de rédacteurs de dissertations ont du mal à citer adéquatement du matériel tiré de livres, d'articles ou d'Internet. En règle générale, vous devez fournir toutes les références aux idées et aux faits qui proviennent d'autres personnes, que ces références soient sous la forme de citations ou reformulées dans vos propres mots. Si vous copiez du matériel (c'est-à-dire, si vous copiez-collez), vous devez mettre ce que vous copiez entre guillemets pour indiquer que les mots utilisés ne vous appartiennent pas et pour révéler votre source.

Si on vous oblige à utiliser un style précis de référence, que ce soit l'adaptation de l'American Psychological Association (APA), la méthode auteur-date ou la méthode traditionnelle, assurez-vous de l'utiliser du début à la fin. Si vous avez le choix du style à utiliser, choisissez-en un seul et utilisez-le partout. En cas de doute, utilisez le style que vous trouvez dans votre manuel de cours ou dans les articles suggérés par votre professeur.

La façon la plus simple de citer un auteur est souvent celle qui utilise le nom de l'auteur et la date de publication (méthode auteur-date). Vous trouverez ci-dessous trois exemples de références auteur-date dans une phrase : 
Un auteur soutient que la retraite est un moment charnière dans la vie des individus et a aussi des effets plus larges sur la société (Klassen, 2013) ;

Klassen (2013) affirme que la retraite «est un événement significatif dans le cycle de la vie; dans un sens plus large, elle a des effets sur le milieu de travail, le marché du travail, l'économie et la société » (p. 1) ;

Selon une position, la retraite a de grands « effets sur le milieu de travail, le marché du travail, l'économie et la société », et touche également les individus et leur famille (Klassen, 2013, p. 1).

Votre bibliographie inclura l'information suivante :

KLASSEN, Thomas R. (2013), Retirement in Canada, Don Mills, ON, Oxford University Press, 176 pages.

Si vous avez trouvé le livre en version électronique, ajoutez le lien au bas de l'information entrée plus haut:

KLASSEN, Thomas R. (2013), Retirement in Canada, Don Mills, ON, Oxford University Press, 176 pages. http://www.amazon.ca/ Retirement-Canada-Thomas-R-Klassen/dp/0199005745.

Indiquez quand vous l'avez lu :

KLASSEN, Thomas R. (2013), Retirement in Canada, Don Mills, ON, Oxford University Press, 176 pages. (consulté le 14 mars 2015) http://www.amazon.ca/Retirement-Canada-Thomas-R-Klassen/ dp/0199005745.

La citation précise et complète des sources est extrêmement importante au gouvernement et dans le milieu des affaires, où une atteinte à la propriété intellectuelle peut mener à des poursuites judiciaires, des sanctions et des congédiements. À tout le moins, un employé qui ne cite pas convenablement ses sources sera rappelé à l'ordre, formellement ou non. 


\section{Lapage-titre}

Au moment de faire vos révisions finales, n'oubliez pas le titre de votre travail. Peu importe sa taille ou son contenu, chaque dissertation ou rapport écrit devrait avoir un titre significatif, unique et attirant. Le titre ne devrait pas simplement reprendre la question du devoir, mais donner au lecteur une idée de ce dont il s'agit. Un titre réfléchi et intrigant prédispose le lecteur à ce qu'il lira avant même de commencer.

La page de titre comprend également de l'information de base, comme la date, le numéro de cours, votre nom et votre numéro étudiant, le nom de votre professeur, de l'assistant à l'enseignement ou du correcteur. Faites-en quelque chose d'intéressant (en incluant un graphique ou une image), étant donné que la page de titre met la table pour ce qui viendra ensuite. Il est toutefois toujours plus prudent de vérifier d'abord si votre professeur a des préférences à cet égard, car certains n'aiment pas les diagrammes, les graphiques ou les polices de caractères inhabituelles.

Si vous croyez que cela ajoutera de la valeur à votre travail, pensez à insérer une citation intéressante sur la première page de votre dissertation. Plusieurs auteurs estiment qu'il s'agit là d'une façon efficace d'attirer l'attention et d'intéresser les lecteurs. Vous aurez peutêtre remarqué que chaque chapitre de ce livre commence par une citation. Toutes ont été choisies soigneusement pour leur signification et pour leur pertinence. Finalement, assurez-vous de numéroter les pages de votre travail.

\section{Les erreurs fréquentes en rédaction de dissertations}

Si vous avez besoin d'améliorer votre grammaire, sollicitez l'aide du centre d'aide à la rédaction des travaux de votre établissement d'enseignement ou d'un tuteur personnel. L'effort en vaut la chandelle, car si vos compétences en rédaction s'améliorent grandement, vos notes feront de même! De plus, la grammaire s'apprend rapidement, ce qui signifie que l'investissement en temps (et en argent), bien que minime, vous rapportera pour le reste de vos jours.

Voici quelques erreurs de rédaction fréquentes, tant aux études que dans le monde professionnel. 


\section{La surutilisation de la voix passive}

La phrase suivante comporte deux exemples de la voix passive : «La réunion a été commencée et le budget a été présenté. » Si on la met à la voix active, la phrase se lit ainsi : « Le président a commencé la réunion et le trésorier a présenté le budget. » Il est parfois difficile d'éviter la voix passive. Sa surutilisation, par contre, diminue la portée et la clarté de vos idées. Les étudiants utilisent souvent les constructions passives pour ne pas avoir à identifier le sujet de leurs phrases. Par exemple, ils écriront: "Cela a été considéré comme un problème important. » Un bon correcteur demandera alors : « Par qui ? »

\section{Le mélange de pronoms}

Vérifiez toujours vos pronoms (les mots comme il, elle, on, ils) pour vous assurer qu'ils s'accordent bien les uns avec les autres. En d'autres termes, ne commencez pas une section avec «ils » pour ensuite passer au «il». Si vous commencez avec un nom singulier, gardez-le jusqu'à la fin. Une phrase comme : «Le monde a remis leurs travaux » est grammaticalement fausse, mais très fréquente. Évitez également d'utiliser les pronoms de manière vague. Par exemple, dire "C'était bien, la nuit dernière » ne donne aucune indication au lecteur sur la nature de ce qui était bien.

\section{Les erreurs de temps de verbes}

La mauvaise utilisation des temps de verbe constitue probablement l'erreur la plus répandue et la plus dérangeante. Elle force les lecteurs à relire les phrases pour s'assurer qu'ils les ont comprises. Si vous commencez à écrire votre dissertation au passé, vous devez continuer au passé (à moins que vous n'ayez une raison logique de changer). Quoi qu'il en soit, ayez toujours conscience du temps de verbe que vous utilisez.

\section{Les phrases alambiquées}

Les phrases alambiquées résultent parfois de la complexité du matériel à l'étude ou de l'esprit embrouillé de l'auteur, mais elles proviennent toutes de la volonté qu'a l'auteur d'avoir l'air savant. Elles sont souvent accompagnées de la voix passive, ce qui les rend encore plus 
compliquées et déconcertantes. Tentez d'être le plus clair possible, épurez vos phrases, débarrassez-vous des fioritures et souvenez-vous que ce n'est pas parce que c'est clair pour vous que ça l'est pour le lecteur.

\section{Les mauvais choix de mots ou les mots manquants}

Les mauvais choix de mots ou les mots manquants, résultats d'une surdose de copier-coller, indiquent habituellement que le travail a été rédigé en quatrième vitesse. Ils ont l'effet d'un avertissement pour votre professeur ou votre employeur : cette personne fait les choses à la dernière minute. Ce qui signifie généralement que vous pratiquez le je-m'en-foutisme...

Faites-nous confiance : vous ne voulez pas donner cette impression. Relisez votre travail pour vous assurer que c'est le mieux que vous pouvez faire.

\section{Les anglicismes}

Il existe plusieurs sortes d'anglicismes :

- les anglicismes intégraux (des emprunts directs à l'anglais, comme background, focus, momentum et timing);

- les anglicismes hybrides (des emprunts auxquels on a ajouté un élément du français, comme speedé, cancellation, focussé et supporteur);

- les anglicismes sémantiques (donner un sens anglais à des termes qui existent en français, comme : « adresser » au sens de « traiter » ou «s'occuper », « éligible » au sens «d'admissible » et «académique» au sens «d'universitaire», «didactique » ou « scolaire»);

- les anglicismes syntaxiques (reproduire une structure anglaise en français, comme : « les prochaines deux semaines » au lieu de « les deux prochaines semaines » ou « siéger sur un comité » au lieu de «siéger à un comité »);

- les anglicismes morphologiques (traduire littéralement une forme anglaise, comme: "à date» pour «to date» au lieu de «à ce jour » ou «jusqu'à maintenant»);

- les anglicismes phraséologiques (emprunter une expression à l'anglais, comme « sonner une cloche » au lieu de «se rappeler quelque chose» ou «gagner son point» au lieu de «avoir gain de cause ». 
Dans le doute, vérifiez! La Banque de dépannage linguistique de l'Office québécois de la langue française est une bonne ressource à cet égard, mais il existe beaucoup d'autres outils pour vous aider à éviter les anglicismes.

\section{L'accord du participe passé}

Les règles d'accord du participe passé donnent bien des maux de tête à quiconque rédige en français. Certains cas d'accord sont, en outre, particuliers et ne suivent pas les règles, selon que les participes sont suivis d'un infinitif ou précédés d'une fraction, d'un nom collectif ou de « en » (pour ne nommer que quelques cas de figure).

En règle générale, le participe passé utilisé avec l'auxiliaire «avoir» s'accorde en genre et en nombre avec le complément d'objet direct si celui-ci est placé avant le verbe, et reste invariable s'il est placé après le verbe ou s'il n'y a pas de complément d'objet direct.

Le participe passé, utilisé avec l'auxiliaire "être», s'accorde quant à lui en genre et en nombre avec le sujet, mais peut demeurer invariable dans les cas de verbes occasionnellement pronominaux (entre autres).

La règle d'or : révisez vos règles de participe passé et vérifiez toujours en cas de doute.

\section{L'accord avec « on »}

L'accord avec le pronom personnel «on» pose parfois problème. Rappelez-vous seulement que, s'il désigne une personne ou un ensemble de personnes dont on ignore l'identité, l'adjectif ou le participe passé qui se rapporte à «on» reste invariable.

$\mathrm{Si}$ « on » désigne une ou plusieurs personnes définies et que vous pouvez le remplacer par un pronom personnel (je, tu, vous, nous), l'adjectif ou le participe passé qui s'y rapporte s'accorde en genre et en nombre avec le sujet que le pronom « on » représente. Par exemple, «On est allées ensemble à la plage » («on » équivalant à un «nous » féminin).

\section{"Ça sonne correct!»}

À l'occasion, les étudiants tenteront de justifier une erreur en affirmant qu'elle était acceptable quand ils étaient au secondaire. Eh bien, nous 
avons des petites nouvelles pour vous: vous n'êtes plus au secondaire! Il n'est jamais agréable de se faire critiquer sur la façon dont on écrit, en particulier si ça ne nous est jamais arrivé, mais le milieu des études postsecondaires et le monde du travail sont très critiques. C'est comme ça.

Les étudiants trouvent parfois que leurs devoirs ont l'air de saigner tellement ils sont barbouillés d'encre rouge! Certains disent que leur professeur ou assistant à l'enseignement est trop sévère. Soyez sûrs d'une chose : vos gestionnaires, vos clients et vos collègues seront beaucoup plus durs et directs quand viendra le moment de vous donner de la rétroaction. Dans la plupart des grosses compagnies ou organisations, les lettres et les documents passent par plusieurs révisions et modifications pour éviter d'avoir l'air de manquer le moindrement de professionnalisme.

Les organismes progressistes peuvent récompenser généreusement ceux qui possèdent de bonnes habiletés en rédaction. Les bons rédacteurs, comme les bons penseurs, sont de plus en plus rares. C'est pourquoi de bonnes habiletés en rédaction pourraient vous mener loin dans la profession de votre choix.

\section{Les rapports de laboratoire et autres devoirs du genre}

Bien que les sections ci-dessus traitent principalement des dissertations, beaucoup des commentaires formulés s'appliquent à d'autres types de travaux que vous devrez rédiger au cours de vos études postsecondaires, qu'il s'agisse de critiques de livres, ou de journaux, ou d'études de cas.

L'exception qui confirme cette règle est le rapport de laboratoire. Son format, généralement préétabli, est plutôt restrictif. Assurez-vous donc de savoir ce qu'on attend de vous et n'en dérogez pas.

Les études de cas ont un style plus concis et professionnel, et sont organisées d'une manière qui leur est particulière. Elles progressent rapidement de descriptions en analyses pour en arriver à la meilleure solution possible. Elles laissent toutefois la place à l'exploration d'options et à l'exercice de la pensée critique et de l'imagination.

\section{Le mot de la fin}

Ce chapitre vous a fourni des outils et des suggestions précises pour améliorer votre façon de faire des examens et des devoirs écrits. Vous 
pouvez également les utiliser pour améliorer beaucoup d'autres aspects de vos travaux scolaires. Souvenez-vous toutefois que vous aurez besoin de temps pour développer un style et une méthode qui fonctionnent pour vous et qui donnent les résultats escomptés. Plus vous perfectionnerez votre style ou votre méthode pendant vos études, plus vous en profiterez après avoir obtenu votre diplôme.

Disneyland ne s'est pas bâti en un jour et vous ne passerez pas maître dans l'art de rédiger du jour au lendemain, mais, sur le marché du travail, peu de compétences portent plus de fruits que la capacité à exprimer des idées complexes par écrit.

\section{Notes}

1. Adapté et utilisé avec la permission de l'Université York, Learning Skills Service.

2. Les sept qualités sont adaptées du livre de T.G. Gebremedhin et L.G. Tweeten, Research Methods and Communication in the Social Sciences, Westport, CT, Praeger, 1994. 
Page blanche conservée intentionnellement 


\title{
Améliorer son sens critique
}

\author{
«Par trois méthodes, nous pouvons \\ apprendre la sagesse : tout d'abord, grâce à \\ la réflexion, qui est la plus noble. En second \\ lieu, par imitation, qui est la plus facile; et \\ la troisième par l'expérience, qui est la plus \\ amère. »-Confucius
}

$\mathrm{D}$ e nos jours, les organisations, peu importe leur taille, ont besoin d'employés qui peuvent faire plus qu'exécuter, de façon compétente, les tâches qu'exige leur emploi. Elles ont besoin de joueurs d'équipe qui peuvent partager, filtrer et traiter efficacement l'information. Les compétences que vous devriez développer pendant vos études postsecondaires et qui jouent un rôle clé pour votre succès à long terme sont connues sous le nom de sens critique. Tout employeur progressiste sera à la recherche de compétences professionnelles et de sens critique.

Les véhicules principaux de l'organisation critique du savoir sont les concepts et les théories. Les concepts sont à la base des théories et les théories sont des cadres abstraits qui permettent de comprendre des données. Ensemble, ils constituent des outils puissants pour découvrir le sens. La science économique classique utilise la théorie des marchés. Les concepts qui se trouvent à la base de cette théorie sont l'intérêt personnel, la concurrence, la propriété privée, les profits et le laissez-faire. 
Il y a toutes sortes de concepts et de théories et, à l'image du monde dans lequel nous vivons, ils sont en constante évolution. La capacité à les adopter et à les adapter différencie un simple étudiant ou un professionnel moyen d'un étudiant ou d'un professionnel clairement au-dessus de la moyenne. Les maîtriser en vaut donc la peine!

Cependant, ne mettons pas la charrue avant les bœufs. Avant de creuser davantage les concepts et les théories, nous devons mieux comprendre ce sens critique que vos professeurs et vos futurs employeurs prennent tant au sérieux.

\section{Qu'est-ce que le sens critique?}

Ne vous laissez pas avoir par la terminologie. Certains étudiants croient que faire preuve de sens critique signifie critiquer les choses en permanence. C'est tout le contraire! En fait, le sens critique pourrait tout aussi bien s'appeler sens constructif, même s'il implique également de déconstruire, c'est-à-dire de défaire des structures dépassées.

La tendance à critiquer sans vouloir reconstruire révèle une certaine immaturité intellectuelle. Vos professeurs auront probablement plus de tolérance à l'égard de la négativité que vos employeurs, puisqu'ils savent à quel point il est nécessaire, bien qu'étrange, pour les étudiants de s'interroger sur les idées reçues et la connaissance. Au final, toutefois, la pensée critique doit être constructive et stratégique si vous voulez qu'elle soit utile dans le monde professionnel. L'expression «si vous ne faites pas partie de la solution, vous faites partie du problème » s'applique au sens critique.

Le sens critique relève des outils et des stratégies que nous utilisons pour analyser de manière créative, comparer, synthétiser et communiquer de l'information afin d'éliminer la confusion et de régler des problèmes. Ces aptitudes sont prisées au sein de toute société, mais elles sont essentielles dans le monde d'aujourd'hui, dans lequel les idées intelligentes et l'imagination sont les clés du progrès et du succès. Le but premier des études postsecondaires est de développer ces aptitudes chez les leaders, les professionnels et les citoyens de demain.

Vos études postsecondaires ont pour but de vous aider à développer vos compétences disciplinaires et professionnelles ainsi que votre sens critique. Des trois, c'est le sens critique qui est le plus important pour l'apprentissage, lequel durera toute votre vie parce qu'il s'applique au plus large éventail d'activités possible. Les disciplines 
savantes et les exigences professionnelles changent tout le temps. Les concepts et les théories sont également sujets à changement, mais le sens critique nécessaire à l'organisation des concepts par rapport aux théories demeure constant et s'applique à tous les environnements. Vos compétences professionnelles et disciplinaires vous permettent d'obtenir un emploi, mais le succès que vous y aurez dépend de votre sens critique.

Le sens critique est essentiel à l'organisation humaine et à l'essor organisationnel. Il nous permet :

- d'identifier les problèmes et de prendre des décisions intelligentes;

- de faire des liens et de créer des hypothèses pour éliminer la confusion;

- de comparer et d'opposer les répercussions que peuvent avoir des stratégies précises ;

- d'évaluer et de communiquer une solution favorite ;

- d'agir avec confiance et fermeté.

\section{Communication et pensée critique}

Si la résolution de problèmes est le cœur de la pensée critique, la communication en est l'âme. C'est un sujet tellement important que nous explorerons ici en profondeur le lien entre les habiletés en communication et le sens critique, pour ne parler de la résolution de problèmes qu'aux chapitres 7 et 8 .

Si vous n'êtes pas en mesure de communiquer clairement, vous ne pouvez pas penser de manière critique. Cela semble un peu extrême, n'est-ce pas? Les étudiants se plaignent souvent qu'ils sont traités de manière injuste et inéquitable, en particulier quand ils obtiennent de mauvaises notes pour une dissertation ou une présentation. Cependant, quand nous révisons avec eux leur devoir de manière critique et constructive, il devient habituellement évident qu'ils n'ont pas fait preuve de pensée critique et, en conséquence, n'ont pas bien compris le contenu.

De bonnes habiletés en communication vont de pair avec une bonne aptitude à penser de manière critique, car elles fournissent aux étudiants et aux employés les outils pour circonscrire et résoudre clairement les problèmes. La communication, en particulier sous forme de dialogue ou de débat avec d'autres, est perçue comme le catalyseur principal de la pensée depuis au moins l'époque de la Grèce antique. 
L'invention du papier a par la suite fait de l'écriture le moyen de choix pour développer et véhiculer le sens critique. C'est la raison pour laquelle la rédaction de dissertations demeure fondamentale en éducation postsecondaire.

Le sens critique et de bonnes habiletés en communication ne sont pas faciles à cultiver. C'est pourquoi ils jouissent d'une telle estime dans les établissements postsecondaires et chez les employeurs. Nous, les humains, sommes une espèce incroyablement intelligente, mais nous pouvons également être des créatures mentalement paresseuses, influençables, émotives, esclaves de nos habitudes et bourrées de préjugés. Non seulement devons-nous travailler fort pour développer notre sens critique, mais nous devons travailler encore plus fort pour constamment et continuellement l'appliquer. Le sens critique est comme toute autre habileté que nous acquérons; nous devons nous en servir souvent pour ne pas perdre notre capacité à l'utiliser.

Savez-vous pourquoi ceux qui détiennent un diplôme d'études supérieures ne souffrent pas autant ou aussi tôt de la maladie d'Alzheimer que le reste de la population?

Certainement pas parce qu'ils sont génétiquement supérieurs. C'est plutôt parce que, typiquement, ils exercent une profession ou travaillent pour des organisations qui exigent d'eux qu'ils utilisent les habiletés à communiquer et à penser de manière critique.

Souvent, les étudiants croient que, pour communiquer, il suffit de parler aux autres et qu'avec un peu de chance, ils parviendront à les divertir, à les intéresser ou à les convaincre. Les étudiants sans expérience font souvent l'erreur de communiquer l'information de façon à plaire à leur professeur (ou du moins, ils l'espèrent). Ce qu'ils ne comprennent pas, c'est que la communication n'a pas seulement lieu avec les autres. Apprendre à penser de manière critique signifie apprendre à communiquer avec nous-même.

Nous le faisons tout le temps, mais notre communication avec nous-même comporte beaucoup d'interférences, en plus d'être remplie de significations contradictoires. Quand nous discutons avec nousmême, nos habitudes ou nos émotions entravent habituellement la véritable compréhension. La pensée critique est la méthode à utiliser pour éliminer les interférences et communiquer plus significativement avec nous-même. Dans toute profession, et dans la vie en général, ceux qui ont le plus de succès sont ceux qui ont appris à le faire.

La communication est intimement liée à la pensée critique de trois manières fondamentales : 
1. Les habiletés en communication vous permettent de clairement cerner un problème; si vous êtes en mesure de le faire, vous êtes déjà sur la voie de sa résolution ;

2. Les mots sont des symboles. Ils représentent quelque chose. Plus vous êtes capable de les utiliser pour communiquer, plus profondément vous pouvez approfondir un problème, un dilemme ou une question ;

3. Les habiletés en communication sont nécessaires pour lier les symboles aux théories. Les concepts sont à la base des théories et les théories constituent la façon la plus sophistiquée d'organiser la connaissance.

La plupart des gens abordent les problèmes de manière superficielle. Ils ont tendance à croire ce qu'on leur dit ou ce qui est le plus simple à comprendre. Parce qu'ils n'arrivent pas à penser efficacement de manière abstraite, la plupart d'entre eux sautent trop rapidement à des conclusions simplistes et en apparence évidentes. Leur perte est votre gain. Développer votre sens critique vous permettra de vous démarquer et de fournir de l'information et des analyses à valeur ajoutée à l'école et au travail.

\section{Améliorer son sens critique}

Personne n'a dit qu'il était facile de développer son sens critique. Si ce l'était, ceux qui en font preuve ne seraient pas aussi populaires. Mais beaucoup d'auteurs qui écrivent sur le sens critique compliquent les choses plus qu'il ne le faut en vous lançant un paquet de trucs abstraits et en espérant que vous vous souviendrez de quelques-uns d'entre eux. Une meilleure approche consiste à commencer par reconnaître que vous avez toujours été capable de faire usage de pensée critique; vous ne saviez simplement pas comment utiliser cette aptitude jusqu'à maintenant. Il ne reste plus qu'à vous exercer rigoureusement et systématiquement à exécuter des processus de pensée que vous faisiez intuitivement par le passé.

Dans ce chapitre, nous vous révélons les règles qui se cachent derrière le sens critique pour que vous puissiez vous-même les appliquer, mais, avant de vous aventurer, prenez en considération ce conseil maintes fois éprouvé : pratiquement personne n'apprend le sens critique en mémorisant toutes les règles. Comme le suggère Michael Gilbert : 
Comme quand vous essayez de vous souvenir du nom de quelqu'un: plus vous essayez, plus c'est difficile; mais dès que vous abandonnez, que vous relaxez et que vous arrêtez d'essayer, le nom vous vient à l'esprit... L'information est là, vous l'avez engrangée ; en relaxant et en étant à l'écoute, vous permettez à votre esprit de réagir librement à ce que vous entendez. Un phénomène semblable se produit dans le sport. Vous pouvez apprendre tout ce qu'il y a à savoir sur la meilleure façon de frapper la balle au tennis ou au golf, mais penser à votre bras droit quand vous êtes sur le court ou sur le terrain de golf est la pire chose que vous puissiez faire. Un entraîneur vous recommandera plutôt de relaxer complètement et de faire le vide dans votre esprit ${ }^{1}$. (Traduction libre)

L'apprentissage du sens critique est semblable à celui d'un sport professionnel. C'est difficile, certes, mais ce n'est pas nécessairement désagréable. Peu de choses dans la vie procurent autant de satisfaction que de résoudre un problème mental. Réussir à bien appuyer et étayer un raisonnement dans une dissertation ou à rédiger un rapport efficace est un peu comme réussir un trou d'un coup au golf ou à marquer un but au hockey. Comprendre un problème complexe, que vous aviez peut-être auparavant considéré au-delà de vos capacités, s'apparente à enfin faire un tour du chapeau devant ses partisans.

Une des composantes les plus importantes du sens critique est la capacité à cerner les concepts et les idées. Beaucoup trop d'étudiants et d'employés adoptent une attitude passive et se comportent, en situation d'apprentissage, comme des récipients vides qui attendent d'être remplis.

C'est pourquoi ils ont du mal à différencier les idées clés du reste. Quand vous faites usage de pensée critique, vous devez séparer les concepts importants des exemples ou des éléments qui servent à les appuyer.

Vous utilisez tout le temps des concepts, probablement sans même vous en rendre compte. Les concepts sont des outils symboliques destinés à aider à la compréhension. Ils n'apparaissent pas comme par magie; ce sont des inventions culturelles. Le concept d'adolescence, par exemple, a été créé au xix siècle. Avant cela, les adolescents n'existaient tout simplement pas. Le concept des pairs a été inventé au $\mathrm{xx}^{\mathrm{e}}$ siècle par les spécialistes des sciences humaines. Le terme «mondialisation» était inconnu il y a 30 ans. Les médias 
sociaux, le cyberespace et beaucoup de choses qui sont essentielles à nos vies et à notre monde ont une histoire encore plus courte.

«Les pairs» est une expression ou un symbole qui a une définition. Cependant, si les définitions des dictionnaires et des encyclopédies sont utiles, elles sont habituellement limitées. Un ensemble complexe d'informations savantes et professionnelles sur les pairs a transformé le concept pour qu'il puisse être mieux utilisé de façon analytique. Vous comprenez probablement de façon intuitive qui sont vos pairs, mais connaissez-vous toutes leurs caractéristiques? Les démographes, les spécialistes du marketing, les enseignants, les actuaires, les criminologues et beaucoup d'autres professionnels ont analysé le concept de pairs de fascinantes façons. Par exemple, les spécialistes de la publicité et du marketing essaient, grâce à lui, de manipuler vos sentiments d'insécurité et votre besoin paradoxal d'appartenir à la masse et de vous en démarquer.

Les concepts sont rarement utilisés de manière isolée. Pour les rendre plus utilisables, les scientifiques les organisent habituellement en groupes et les catégorisent selon qu'ils sont fondamentaux (parfois appelés variables indépendantes) ou secondaires (parfois appelés variantes dépendantes). Ce qui est fondamental et ce qui est secondaire dépend habituellement du problème dont il est question. Ainsi, nous pouvons essayer de comprendre la pression exercée par les pairs comme une variable dépendante de l'adolescence ou nous pouvons faire des recherches sur l'adolescence comme période définie de la vie, dans laquelle a lieu la pression par les pairs. D'autres formes de pression exercée par les pairs peuvent avoir lieu dans des foules, des cultes religieux, des environnements de banlieues (la rivalité entre voisins) et ailleurs.

Les groupes de concepts peuvent être relativement simples, comme ils peuvent être très compliqués. Les concepts dans le monde des disciplines collégiales ou universitaires, par exemple, sont habituellement organisés selon des systèmes de classification. Vous pensez peut-être que vous savez déjà ce que signifie un concept comme celui de démocratie, mais nous pouvons vous assurer que vous aurez une compréhension beaucoup plus élaborée de celui-ci si vous suivez un cours en sciences politiques. Par exemple, la démocratie possède au moins trois dimensions distinctes:

- des élections libres où tout le monde a le droit de vote ;

- les libertés d'expression et d'association ;

- un gouvernement contrôlé par un corps législatif élu. 
Dans chacune de ces trois dimensions, et entre elles, se trouvent plusieurs variations différentes que les spécialistes des sciences politiques étudient. En effet, des cours universitaires et plusieurs livres portent entièrement sur la définition de la démocratie.

Vous remarquerez peut-être que la définition de la démocratie présentée ci-dessus n'aborde pas la dimension de la libre entreprise ou du capitalisme, que les gens associent souvent à la démocratie. Les groupes de concepts se fondent invariablement sur des suppositions et sont souvent des partis pris caractéristiques. Cependant, certaines suppositions et certains partis pris peuvent être nécessaires au bon fonctionnement des concepts, alors que d'autres sont complètement injustifiés. Une société véritablement démocratique, par exemple, n'a pas besoin d'être capitaliste : elle peut tout aussi bien être socialiste ou communiste.

\section{Les limites du récit}

Les communautés humaines ont toujours organisé et transmis toute information d'importance sous la forme de récits ou d'histoires. Il n'y a aucun mal à le faire: les récits sont à la fois des façons précieuses d'acquérir des connaissances et d'importantes sources d'information. Ils sont essentiels à l'identité culturelle et personnelle, mais l'utilisation du récit pour construire la connaissance présente des inconvénients de taille.

Les étudiants plus jeunes tentent souvent de donner du sens à l'information en la présentant sous forme de récit. Lors de la rédaction d'un travail de recherche, ils présentent l'intrigue de base de façon très habile et essaient souvent d'ajouter de la valeur en mettant beaucoup de détails, mais relativement peu de débutants parviennent à bien disséquer et mettre en lumière le sens fondamental ou la signification de l'histoire.

La plupart de nos étudiants aiment bien regarder des films. C'est pourquoi nous y faisons référence quand nous enseignons le sens critique. Vous pouvez regarder un film de manière superficielle ou vous pouvez le regarder de manière critique. Un bon film devrait communiquer différents niveaux de sens. Au moins quelques-uns de ces niveaux seront invisibles à quiconque souhaite seulement être diverti par une bonne histoire. Un grand film, de la trempe de ceux qui passent à la postérité, remettra en question vos préjugés en fournissant des niveaux de sens subtils et complexes. Si vous avez l'habitude 
de penser de manière critique, vous trouverez probablement qu'un film qui ne repose que sur une histoire d'amour ou une comédie de base ne fait qu'insulter votre intelligence.

Prenez, par exemple, le film Une jolie femme (Pretty Woman), mettant en vedette Julia Roberts et Richard Gere. Quoique les acteurs jouent bien et que le film soit relativement plaisant, il ne veut pas dire grand-chose parce qu'il ne communique rien de profond ou d'intéressant. Il débite des banalités, comme l'idée que l'amour peut vaincre tout, que chacun trouve sa chacune et que nous avons tous des chances égales d'être heureux. Cette combinaison est en fait vaguement plausible uniquement pour ceux qui ressemblent à Julia Roberts et qui ont la chance de trouver un bienfaiteur millionnaire. En outre, la plupart des millionnaires ressemblent plus souvent à Bill Gates et à Donald Trump qu'à Richard Gere.

Quand nous disons de telles choses, nos étudiants nous accusent de cynisme. Ils soulignent, avec raison, que la plupart des films comportent des histoires plaisantes, des rêves qui exigent que nous mettions de côté, pour un instant, notre incrédulité. C'est tout à fait acceptable comme approche; cela démontre un certain degré de séparation critique. Cependant, beaucoup de gens intériorisent ces rêves et finissent par faire de très mauvais choix de vie ou de carrière. Quand ils se rendent compte que leur vie n'est pas à l'image de celles qu'ils voient dans les films, certains ont l'impression de l'avoir ratée. Il leur manque souvent le sens critique qui leur permettrait d'affronter efficacement la réalité.

Beaucoup de nos étudiants ne perçoivent pas, au départ, que la plupart des films hollywoodiens ne sont rien de plus que du divertissement superficiel. L'histoire d'Une jolie femme s'inspire vaguement d'une pièce de George Bernard Shaw (1856-1950) intitulée Pygmalion. La pièce de Shaw offre des niveaux de sens beaucoup plus subtils et ambigus qu'Une jolie femme. L'un d'entre eux est que les aspirations romantiques ne sont pas nécessairement comblées et qu'elles peuvent même être idéologiques, car elles masquent ou excusent des problèmes fondamentaux au sein de la société. De plus, Shaw soutient que les inégalités économiques et sociales doivent être prises au sérieux si on veut que le terme «égalité des chances » ait une véritable signification. Il n'est pas nécessaire d'être d'accord avec Shaw pour reconnaître qu'il donne à réfléchir.

Pour penser de manière critique, il est nécessaire de situer les concepts clés et les préjugés, et de reconnaître qu'ils peuvent être à la 
fois complexes et multiples. En d'autres termes, vous devez être prêt à travailler et à creuser profondément la matière pour trouver les pépites qui ne sont pas immédiatement évidentes. Une jolie femme, par exemple, n'offre rien de plus que du divertissement passif. Par contre, vous pouvez être certain que vos lectures et vos devoirs auront de la substance.

Être critique signifie reconnaître et évaluer les niveaux variés de sens. Les véritables penseurs critiques peuvent faire la liste de leurs films préférés et expliquer les raisons derrière leur choix. Pratiquement tous les critiques de films pensent que Citizen Kane (1941) est un des plus grands films américains jamais tournés. Pourquoi ? Parce que le film, réalisé, coécrit et produit par Orson Wells (1915-1985), et dans lequel il tient le rôle principal, livre beaucoup de niveaux de sens. Il s'inspire de l'histoire de William Randolph Hearst (1863-1951), un grand magnat de la presse américaine. C'est également un drame moral qui montre comment le pouvoir et la richesse peuvent corrompre. C'est une allégorie de l'essor de la nation américaine, de l'innocence à la complicité. C'est une étude toute en ambiguïté, qui met en évidence la complexité d'un être humain plutôt que d'en donner une image stéréotypée, noire et blanche. Finalement, c'est un chef d'œuvre artistique qui utilise les métaphores de manière subtile pour communiquer des messages complexes.

Plus vous regardez Citizen Kane, plus vous retirez du film. En fait, la plupart des gens ne détectent pas tous les niveaux de sens à la première écoute. Le plus récent film hollywoodien ou bollywoodien est probablement plus divertissant que Citizen Kane, mais il n'arrive pas à la cheville de celui-ci quant à la profondeur, à la technique et au sens.

\section{Mettre l'information en contexte}

Être en mesure de définir les concepts et les idées clés est important, mais vous devez également les comparer et les mettre en contexte. Dans Peter Pan, la pièce de théâtre originale de James Matthew Barrie (1860-1937), ainsi que dans les diverses adaptations cinématographiques qui en ont été faites, vous trouverez les idées clés suivantes:

- L'imagination créative est importante ;

- Les gens sont foncièrement bons et l'enfance est une période d'innocence et de potentiel imaginatif ;

- Les adultes doivent conserver leurs qualités d'enfant ;

- Les enfants doivent grandir et être responsables de leurs actes. 
Dans Peter Pan, la pièce de 1904, l'idée clé, ou le message principal, est le numéro quatre. Dans Capitaine Crochet (Hook), le film de 1991, c'est le numéro trois. Dans Voyage au pays imaginaire (Finding Neverland), le film de 2004, la signification clé est le numéro deux. Ce changement de perspective met l'accent sur des significations très différentes, même si l'intrigue demeure facilement reconnaissable.

La différence entre la pièce Peter Pan et les films Capitaine Crochet et Voyage au pays imaginaire nous mène à une composante importante du sens critique : l'évaluation du contexte de l'information. Les idées et les concepts clés sont de l'information d'avant-plan, mais vous devez souvent prendre en considération l'arrière-plan pour les mettre au jour.

L'arrière-plan peut prendre des formes variées. Dans le cas de Peter Pan, vous étudierez peut-être l'intention de l'auteur. Barrie était un Écossais vivant à une époque où l'Empire britannique était toutpuissant et où les valeurs idéales de la société étaient patriarcales et militaires. Il percevait peut-être l'enfance comme une période merveilleuse et formatrice (alors que les idées morales sont construites), mais il croyait probablement que les adultes (en particulier les hommes) devaient être responsables d'eux-mêmes et jouer un rôle actif dans la gouvernance d'un empire d'une telle envergure. Il y a un temps pour être enfant et il y a un temps pour être adulte.

Capitaine Crochet a été réalisé à une période historique très différente. Dans les années 1990, beaucoup d'Américains avaient l'impression que leur vie devenait bureaucratique, matérialiste et décevante. Ils aspiraient à un retour aux valeurs ostensiblement innocentes de l'Amérique rurale (telle qu'interprétée par Walt Disney). Ainsi, le Peter Pan de Capitaine Crochet, maintenant avocat d'entreprise et incarnation humaine de l'échec total, redécouvre son innocence et son imagination d'enfant. Les lieux et les personnages du film Capitaine Crochet sont essentiellement les mêmes que dans la pièce de Barrie, mais le message ou le sens principal ne pourrait pas être plus différent. Voyage au pays imaginaire, tourné peu de temps après les attentats du 11 septembre 2001, met l'accent sur le rôle de l'amitié et de la famille, et sur la bonté des relations interpersonnelles.

Le contexte comprend plusieurs éléments qui peuvent vous donner des indices sur la signification. Ceux-ci peuvent être de nature historique (la date à laquelle une œuvre a été composée), de nature littéraire ou scientifique (le style ou le genre d'une œuvre), de nature biographique (ce que nous savons de la vie de l'auteur et de ses positions sociopolitiques). 
L'exploration du contexte n'est qu'une des multiples façons de trouver le sens et elle peut parfois être trompeuse. De plus, elle limite souvent la compréhension et l'appréciation que nous avons d'une problématique ou d'une œuvre. Les symboles sont un des moyens les plus répandus de donner des indices sur le sens. Ceux-ci véhiculent des significations complexes, subtiles ou ambiguës, et sont généralement plus fréquents dans les œuvres littéraires que dans les articles de sciences sociales. Bien qu'il ne manque pas de symboles dans le domaine des sciences sociales, les exigences de l'analyse systématique en limitent généralement l'utilisation. Dans les travaux qui reposent davantage sur l'imagination, les symboles jouent le rôle de métaphores.

Par exemple, dans son roman La promenade au phare (To the Lighthouse), Virginia Woolf (1882-1941) fait continuellement référence à différents types de vagues dans l'océan. Parfois, les vagues ne sont que de simples ondulations et tout semble tranquille et serein. Parfois, les vagues sont clapoteuses et différentes les unes des autres. À un autre moment, elles s'abattent violemment sur le rivage. Pour comprendre leur signification, vous devez réfléchir aux symboles et, utilisant votre sens critique, vous vous rendrez compte que les vagues ont davantage à voir avec la signification du roman qu'avec l'histoire. Les êtres humains sont en relation les uns avec les autres dans l'océan social, mais les vagues font aussi référence à l'isolement qui divise les individus. En conséquence, les êtres humains sont parfois connectés les uns aux autres, parfois séparés. À l'occasion, ils s'entrechoquent (comme au cours de la Première Guerre mondiale, dont il est brièvement question dans le livre) et la communauté humaine est transformée en un océan déchaîné et dangereux.

Ainsi, le symbole des vagues met au jour une couche de sens dans La promenade au phare. Si vous y avez porté attention, vous aurez une bien meilleure compréhension de ce dont il est question dans cette grande œuvre littéraire, d'autant plus que Virginia Woolf s'y concentre intensément sur le monde intérieur de ses personnages, au point de parfois rendre l'intrigue insignifiante.

Dans les grandes œuvres qui utilisent une approche narrative plus traditionnelle, vous constaterez tout de même qu'une attention particulière a été portée aux symboles. Par exemple, dans Citizen Kane, le dernier mot que prononce le protagoniste (le personnage principal) sur son lit de mort est «rosebud» (bouton de rose). Si vous regardez attentivement le film, vous découvrirez que Rosebud est le nom qui 
était inscrit sur son traîneau quand il était enfant, avec tout l'avenir devant lui.

Pour découvrir le sens de n'importe quel livre, article, dissertation ou œuvre d'art, il est également important de vous attarder au titre. Les auteurs littéraires y utilisent souvent des symboles clés. Un autre roman de Virginia Woolf s'intitule simplement Les vagues (The Waves). Le titre du roman de Sylvia Plath (1932-1963), La cloche de détresse (The Bell Jar), en dit long sur le message qu'elle veut transmettre. Une cloche est ici un dôme de verre inversé dont les scientifiques se servent pour mesurer combien de temps mettront les souris ou les insectes à respirer tout l'oxygène qui se trouve dans cet espace fermé. Le roman de Plath met en scène une femme extrêmement intelligente qui vit dans une société où les femmes n'ont pas le droit de développer leur propre identité et finissent souvent avec des hommes qui les répriment. Vous voyez le lien ? Pourquoi croyez-vous que Plath est une si grande inspiration pour les écrivains féministes?

Des symboles importants se trouvent même dans les travaux qui semblent purement scientifiques. Nicolas Copernic (1473-1543) renvoie à l'image grecque de la lanterne au centre du ciel pour aider les lecteurs à imaginer un monde où les planètes tournent autour du Soleil. Pour aider ses lecteurs à visualiser le fonctionnement de l'économie de marché (dans lequel l'offre et la demande sont en équilibre), Adam Smith (1723-1790) utilise l'image, ou la métaphore, de la main invisible. Ce symbole évoque l'idée que l'offre et la demande s'équilibrent mutuellement seulement si le gouvernement s'abstient de se mêler de ce qui se passe dans le marché.

Smith avait besoin d'être appuyé par ce symbole parce qu'en 1776, quand il a écrit La richesse des nations (The Wealth of Nations), pratiquement personne n'avait même rêvé qu'une économie de marché fût possible, et la plupart des gens avaient du mal à se l'imaginer. Dès qu'un symbole est utilisé, d'autres penseurs peuvent ensuite l'adopter. Beaucoup d'économistes voient la main invisible de Smith comme la représentation par excellence de leur discipline, alors qu'il l'a peut-être utilisée pour simplement étayer sa préférence pour les marchés moins contrôlés. Pour comprendre ce que Smith affirme, vous devrez donc probablement prendre en considération son symbole et son contexte.

Vous voudrez peut-être également reconnaître que les écrivains retravaillent ou inversent parfois les symboles. L'historien de l'économie, Alfred Chandler (1918-2007), fait allusion au symbole de Smith dans le titre de son livre La main visible des managers (The Visible Hand 
- The Manegerial Revolution in American Business). De quoi croyez-vous qu'il traite? Qu'en pensez-vous? Comment fonctionne l'économie moderne? Qui a le pouvoir de diriger et de manipuler le marché ? Le message de Chandler est le suivant: nous n'évoluons plus dans un libre marché et les multinationales contrôlent de plus en plus nos vies et les risques du marché.

\section{La signification des théories}

Alfred Chandler avait un point de vue. Il pensait que les sociétés modernes sont trop grosses et trop puissantes. Adam Smith avait un point de vue. Il croyait que le progrès n'est possible que si on force les capitalistes à se faire concurrence sur les marchés, sans que le gouvernement intervienne outre mesure. Virginia Woolf voulait conseiller à ses lecteurs de vivre le moment présent et de profiter des petits plaisirs de la vie, puisqu'ils sont les choses les plus humaines et tangibles dont ils peuvent faire l'expérience dans un monde déroutant et occasionnellement chaotique. Sylvia Plath avait aussi une perspective, ou un point de vue. Elle était une écrivaine féministe à plusieurs égards, mais aurait probablement rejeté l'étiquette «féministe». Elle croyait que les femmes intelligentes sont étouffées par une société dominée par les hommes.

Un point de vue, ou une perspective, est une théorie. Les théories ne sont donc pas intrinsèquement mystérieuses. Il s'agit simplement d'abstractions utiles qui combinent, organisent et distinguent des concepts qui sont reliés à des fins d'explications. En fait, vous utilisez constamment des théories. Si vous pensez qu'il faut que Bill Gates soit intelligent pour être aussi riche, vous faites appel à la théorie (douteuse ou non) selon laquelle tous les bien-nantis sont intelligents. La même chose se produit quand vous dites : «Si tu es si intelligent, pourquoi n'es-tu pas riche? » Les données ne viennent pas nécessairement appuyer votre théorie, toutefois.

Une théorie, dans le contexte des études postsecondaires, se compose de données et d'affirmations. On s'attend des érudits qu'ils soient méticuleux dans leur traitement de l'information, qu'ils limitent leurs affirmations à celles qui s'appuient sur des faits et qu'ils mettent continuellement à l'épreuve leurs idées abstraites en les appliquant à des données concrètes. Habituellement, ils utilisent le mot «théorie » seulement si les explications qu'ils donnent sont hautement cohérentes, solidement argumentées et logiquement justifiées. 
Les théories utilisées en sciences humaines (les sciences sociales et les humanités) sont généralement différentes de celles qu'on trouve en sciences dures (chimie, physique, etc.). En sciences dures, beaucoup de théories (pas toutes et pas les plus importantes) prédisent un résultat, alors qu'en sciences humaines, elles visent généralement à l'expliquer. Cependant, les théories ont beaucoup plus en commun qu'elles ne diffèrent: essentiellement, elles tentent toutes de découvrir les causes premières ou les causes plus importantes de certains événements ou résultats. En d'autres termes, elles tentent de trouver un sens à une foule d'informations qui, autrement, seraient beaucoup moins compréhensibles ou utiles.

L'acquisition du sens critique signifie non seulement aller plus en profondeur dans sa façon de penser, mais penser avec plus d'expertise. Faire preuve d'expertise suppose que vous avez une boîte à outils ou des techniques qui vous aideront à approfondir davantage votre pensée, à la recherche de diamants. Les outils les plus précieux pour explorer des données sont les approches ou cadres théoriques. Vous trouverez plusieurs d'entre eux au sein des disciplines scolaires ou des professions. Disons, par exemple, que vous voulez comprendre comment le sens critique se développe habituellement chez vos camarades de classe. Pour y parvenir, vous voudrez peut-être adopter un cadre théorique développé par Benjamin Bloom. La théorie de Bloom va comme suit :

1. Les étudiants en première année d'études postsecondaires ont tendance à voir le monde en noir et blanc. Ils veulent que leurs professeurs leur disent ce qui est bien et ce qui est mal. Ils absorbent passivement l'information sans la critiquer ;

2. En deuxième année d'études postsecondaires, les étudiants commencent à comprendre que le mot clé est " gris » au lieu de «noir et blanc». À ce point, ils savent que les sens peuvent être multiples et que les faits ont besoin d'être interprétés. Par contre, même s'ils comprennent que les points de vue ont un certain poids, ils ne sont pas très habiles dans la manipulation et l'utilisation des cadres théoriques. Ils semblent penser qu'ils ont droit, comme tout le monde, à leur opinion ;

3. En troisième année, les étudiants commencent à utiliser les cadres théoriques plus adroitement et apprennent à appuyer leurs arguments par la logique et des preuves. Il leur manque probablement toujours un monde de sens personnel avec lequel ils pourraient traiter l'information qu'ils reçoivent. Ils 
ont appris à penser de manière critique, mais dépendent toujours de leurs professeurs ;

4. Avec un peu de chance, les étudiants qui sont en quatrième année d'études postsecondaires ont développé leur propre cadre théorique, qu'ils appliquent de manière autonome dans leurs études. Ils reconnaissent la diversité, mais peuvent former et intégrer le matériel à leur manière. Ils ne dépendent plus de l'opinion et des approches de leurs professeurs dans leur façon d'aborder les textes et autre matériel ${ }^{2}$.

Les théories sont des façons systématiques d'interpréter ou de donner un sens à la réalité. Même si elles ont un pouvoir explicatif ou prédictif, elles représentent rarement des vérités absolues, même dans des champs d'études comme la physique et la chimie. Elles sont plutôt des manières utiles d'organiser de l'information complexe. Ce ne sont pas tous les étudiants de première année qui se conformeront au stéréotype de Bloom. Et ce ne sont pas tous les étudiants de quatrième année qui seront aussi matures et indépendants qu'il l'espère.

Ce n'est pas parce que les théories sont des outils importants qu'elles sont immunisées contre l'analyse critique. Les chercheurs féministes, par exemple, ont critiqué le modèle de Bloom parce que ce dernier préférerait un sexe. Pouvez-vous deviner comment un ou une féministe peut critiquer le modèle de Bloom ? Pouvez-vous penser à un autre modèle ou à une variation de son modèle? Si c'est le cas, vous manifestez déjà une capacité à penser de manière critique.

\section{Niveaux et formes théoriques}

Les cadres théoriques prennent une multitude de formes et fonctionnent à plusieurs niveaux. En termes simples, certains sont des métathéories - de grandes constructions englobantes qui expliquent d'énormes pans de la réalité. D'autres sont des microthéories qui expliquent de petits phénomènes localisés. Certains fonctionnent au niveau "méso ", médian, et font habituellement le lien entre les métathéories et les microthéories. On utilise généralement soit les métathéories, soit les microthéories, mais les mésothéories sont parfois utiles pour expliquer une singularité ou un problème au sein d'une métathéorie.

Est-ce que vous vous y retrouvez ? Voici un exemple. Une métathéorie clé de l'œuvre de Karl Marx (1818-1883) affirme que la classe ouvrière (ceux qui sont employés par d'autres) se soulèvera dans un 
conflit révolutionnaire contre ceux qui possèdent les moyens de production et la richesse (les employeurs). Si vous voulez conserver la théorie, mais expliquer pourquoi ce genre de révolution n'a pas eu lieu au cours du $\mathrm{xx}^{\mathrm{e}}$ siècle, vous voudrez peut-être vous servir d'une mésothéorie. Elle pourrait vous aider à expliquer que la révolution fut court-circuitée par les employeurs et les gouvernements, qui utilisèrent des récompenses (comme des hausses de salaire, le système de santé public et l'assurance-emploi) et des mesures de contrôle (surveillance et menaces de faire appel à des sous-traitants) pour empêcher les travailleurs de s'organiser et de renverser leurs oppresseurs.

Le modèle de l'évolution de l'étudiant de Bloom est une microthéorie à un niveau plutôt bas, une adaptation d'une métathéorie créée par le psychologue Jean Piaget (1896-1980). En sciences humaines et sociales, les grandes métathéories, tout englobantes, sont des façons fondamentales d'organiser la connaissance et de comprendre les données. Voici quelques exemples de métathéories : le marxisme, le freudisme, le féminisme, l'existentialisme, le pragmatisme, le déconstructivisme et le postmodernisme. Il y en a une foule d'autres. Ces très grandes théories incorporent souvent plusieurs aspects de l'existence humaine, comme la psychologie, la spiritualité, l'économie, la philosophie, la sociologie et l'éthique. Elles sont parfois compatibles les unes avec les autres, mais, le plus souvent, elles sont mutuellement exclusives.

Les métathéories seront toujours populaires et porteuses d'un facteur de « coolitude » parce qu'elles ont un grand pouvoir explicatif et peuvent complètement transformer notre façon d'aborder les données ou la réalité (elles sont souvent contraires à l'intuition). De plus, bien les appliquer requiert une bonne dose de sens critique.

Certains chercheurs consacrent leur carrière entière à l'exploration de la théorie du ça, du moi et du surmoi de Sigmund Freud (1854-1939). D'autres continuent de raffiner et d'adapter la théorie du matérialisme dialectique de Marx. Il y a même une école de spécialistes de Jacques Derrida (1930-2004), dans les départements de littérature, qui tenteront de vous convaincre qu'il n'y a pas d'auteur, mais seulement des textes abandonnés qui laissent différentes traces de sens.

On ne s'attend pas à ce que vous deveniez des experts de ces théories pendant vos études, mais apprendre à penser de manière critique impliquera que vous absorbiez quelques concepts et approches provenant de différentes théories, si ce n'est que parce qu'elles sont de 
bons outils. Par exemple, Karl Marx suggère de comprendre l'histoire humaine en tant que lutte entre les classes sociales. Si vous utilisez cette approche théorique, vous pourrez expliquer plusieurs événements historiques, comme les guerres, les révolutions, les grèves, les protestations, les attentats, etc.

Sigmund Freud a mis au jour le pouvoir du subconscient. Vous trouverez plusieurs preuves de son influence dans les romans contemporains et dans des films comme Memento, qui utilisent des techniques en lien avec le flux de conscience et les flash-backs mentaux pour explorer l'esprit. Vous n'avez pas besoin de comprendre parfaitement l'existentialisme ou ce que Jean-Paul Sartre entend par " conscience » pour avoir une idée de certaines de ses perspectives au sujet de nos crises actuelles sur le sens. Quiconque a déjà fait des choix éthiques difficiles ou a observé comment nous excusons hypocritement notre comportement égoïste, ou a déjà eu l'impression que «l'enfer, c'est les autres », est redevable au canon (l'ensemble des théories) existentialiste.

À ce point-ci, vous vous demandez peut-être ce que tout cela a à voir avec la vie après les études. Si c'est le cas, vous n'avez pas encore complètement saisi l'énorme pouvoir de la pensée critique. À tout le moins, être capable d'utiliser des théories avec confiance implique que vous soyez en mesure de vous détacher du flot grandissant et écrasant d'informations, et de former des abstractions utiles. Être à l'aise avec les théories fera de vous quelqu'un qui peut trier et traiter l'information beaucoup plus efficacement, à l'ère où il est essentiel d'être capable de le faire. Même si vous n'êtes pas d'accord avec certaines parties d'une théorie, vous vous rendrez souvent compte que certaines théories sont indispensables.

Les grandes théories sembleront inutiles si vous ne savez pas comment, quand et où utiliser certains de leurs outils. Par exemple, les chefs d'entreprise auraient beaucoup à apprendre de Sartre en ce qui concerne les décisions qu'ils prennent et la responsabilité qu'ils ont quant à celles-ci. Il pourrait également montrer aux critiques des entreprises comment certains dirigeants sont habituellement de mauvaise foi à l'égard des actionnaires et des autres parties prenantes. Les théories de Max Weber (1864-1920), au sujet des grandes organisations, et les analyses de Freud, sur la civilisation, peuvent aider quelqu'un qui travaille au département des ressources humaines à comprendre pourquoi les employés sont mécontents dans des environnements trop bureaucratiques et axés sur les règles, et pourquoi les employeurs auraient intérêt à porter une plus grande attention à la 


\section{Quatre théories majeures en sociologie}

Tout champ d'études ou discipline scolaire a ses théories préférées. Vous trouverez ci-dessous quatre exemples importants en sociologie. Nous avons mis en italiques les concepts clés.

Le fonctionnalisme structurel: Selon cette métathéorie, la société est un système complexe composé de plusieurs structures, tels la famille, l'école, le gouvernement et les employeurs, et chacune a une fonction particulière qui permet d'atteindre un équilibre consensuel. Traduction : La société moderne est à la fois juste et efficace.

Théorie du conflit: Selon cette métathéorie, la société est une combinaison fondamentalement injuste et inégale de classes qui ont des intérêts opposés et dans laquelle la classe dominante exploite le travail des autres.

Traduction: La société moderne est injuste pour la plupart des gens et, en fin de compte, dysfonctionnelle.

Théorie de l'échange et de la réciprocité: Cette microthéorie suggère que les interactions humaines ont toutes une composante économique, qui incite les individus à agir par intérêt personnel pour obtenir des récompenses, y compris l'approbation et l'affection.

Traduction: Chacun pour soi; la vie est un processus de négociation.

L'interactionnisme symbolique: Selon cette microthéorie, les individus construisent leur identité et créent du sens en interagissant avec les autres. Ces interactions incluent les jeux de rôle et l'utilisation de symboles (principalement, mais non exclusivement la langue).

Traduction: Qui nous sommes dépend entièrement de nos interactions avec les autres (famille, amis, enseignants, etc.).

santé mentale de leurs employés. Marx est essentiel à quiconque veut découvrir pourquoi les employés ne font pas confiance à leurs patrons et se sentent aliénés au travail. Même un auteur aussi ésotérique que Derrida, si enseigné correctement et avec un minimum de jargon, peut aider ceux qui travaillent dans des environnements multiculturels et internationaux pour qu'ils passent outre à leur perspective eurocentrique et apprennent à respecter et à accueillir la diversité culturelle. 
Les nouveaux étudiants sont souvent frustrés par les différences significatives entre certaines perspectives théoriques importantes. Ils veulent savoir laquelle est «la bonne». Ces différences peuvent toutefois constituer des avantages pour qui s'intéresse à l'aspect pratique des théories. Mettre en application des perspectives théoriques variées dans le cas d'un problème particulier est une manière extrêmement efficace de découvrir des angles intéressants, des possibilités stratégiques et des solutions novatrices. Vous pouvez, en effet, utiliser une théorie pour clarifier des problématiques et régler des problèmes sans adhérer à celle-ci dans son ensemble.

Souvenez-vous qu'aucune perspective théorique ne peut capturer à elle seule la complexité de la vie humaine et des relations interpersonnelles. Le monde et chacun des individus qui le composent sont trop complexes pour qu'une seule théorie soit en mesure de les expliquer. Cette complexité suggère, en effet, que des perspectives multiples sont nécessaires pour arriver à nous comprendre nous-même ainsi que notre environnement. Ceux qui utilisent une seule approche théorique pour tout expliquer ont peut-être l'air profond, mais ils sont souvent inflexibles et unidimensionnels.

Même si vous n'avez pas souvent la chance d'utiliser formellement les métathéories après vos études, vous pouvez facilement transférer à n'importe quelle profession l'aptitude à interpréter et la profondeur de pensée qu'elles nécessitent. Plus votre aptitude à penser de manière critique est développée, plus vous aurez de chances et de succès sur le marché du travail. Il va sans dire, toutefois, que vous devrez connaître les microthéories et les macrothéories pertinentes à votre emploi.

$\mathrm{Au}$ cours des 40 dernières années, les gestionnaires ont dû apprendre plusieurs nouvelles théories sur la pratique de la gestion d'une compagnie. En fait, il est difficile d'envisager une occupation stimulante future dont le succès ne dépend pas de la mise à jour continuelle des connaissances. Cela signifie également que vous devez toujours vous tenir au courant des plus récentes approches théoriques dans une profession ou une occupation donnée.

C'est pourquoi il est important de commencer dès maintenant à combiner théorie et pratique. Cherchez des façons d'appliquer les plus récentes théories à des problématiques pratiques de la vie de tous les jours. Cela peut inclure les questions d'éthique dans le monde politique et dans celui des affaires, l'impact de la technologie, l'influence de la mondialisation, la signification de l'amour et beaucoup plus encore. Les diplômés qui mettent en pratique leurs connaissances théoriques 
sur le marché du travail et qui restent au courant des développements dans leur domaine sont de véritables atouts pour leurs employeurs.

Vos professeurs n'aspirent pas à vous transformer en théoriciens de l'abstrait (même si cela peut parfois en avoir l'air). Voici un petit secret: même les professeurs peuvent avoir du mal à demeurer au courant des nouvelles théories, mais nous vous garantissons qu'avec de la pratique, vous les utiliserez de mieux en mieux, que ce soit en combinaison ou pour des situations ou des problèmes particuliers.

\section{Trucs pratiques}

D'aucuns pensent, naïvement, que nous naissons avec une certaine quantité d'intelligence et donnent beaucoup trop d'importance aux résultats de quotient intellectuel (QI) et aux moyennes pondérées cumulatives (MPC). Bonne nouvelle pour quiconque s'est déjà senti stupide: la vraie intelligence est la capacité à mettre en pratique la pensée critique, mais encore c'est quelque chose qui s'apprend! Si vous avez bien lu ce chapitre, vous êtes déjà sur la bonne voie.

Voici un petit test: savez-vous pourquoi Blade Runner, 2001, Odyssée de l'espace (2001: A Space Odyssey), voire La matrice (The Matrix) (l'original, pas les films subséquents) sont meilleurs que les films de Star Wars? Savez-vous pourquoi l'album Sgt. Pepper des Beatles ou OK Computer de Radiohead véhiculent plus de sens que n'importe quoi de Kid Ink, Justin Bieber ou Britney Spears ? Être en mesure de comprendre et d'expliquer les différences, c'est de la pensée critique. Cela ne veut pas dire que vous devez préférer les Beatles à Bieber. Tout ce que vous devez faire, au fur et à mesure que vous développez votre goût critique, c'est apprendre à distinguer et avoir conscience qu'il existe du matériel plus sophistiqué, plus profond, et d'autre matériel qui l'est moins ou pas du tout.

L'acquisition du sens critique ne se fait pas en criant lapin. C'est un processus d'apprentissage graduel. C'est pourquoi les études postsecondaires durent généralement plusieurs années ; elles sont conçues pour développer votre sens critique. Trop de professeurs semblent croire, toutefois, que les étudiants vont l'acquérir par osmose. Si vous êtes en contact avec eux assez longtemps, leurs aptitudes finiront bien par déteindre! Nous vous conseillons tout de même de faire preuve d'un peu plus d'initiative et de méthode dans votre approche.

La première étape est de faire des connexions entre ce que vous savez déjà et ce que vous apprenez à l'école. Tout le monde doit 
commencer quelque part et l'expérience personnelle est le meilleur endroit pour ce faire. Quand vous découvrez du nouveau matériel, la meilleure chose à faire est de voir comment il vous touche. Qu'est-ce que cela signifie pour vous? Êtes-vous d'accord ? Si oui, pourquoi? Sinon, pourquoi ? Vous apprécierez mieux l'information plus sophistiquée si vous comprenez comment elle peut vous toucher.

Cependant, ce n'est là qu'un point de départ. Trop d'étudiants se laissent impressionner par le matériel plus complexe parce qu'ils le trouvent difficile, étranger et exigeant. Ils ont tendance à le simplifier à outrance pour se sentir plus à l'aise ou à le rejeter parce qu'il est différent de ce qu'ils ont vu auparavant. Dans de telles situations, il est impératif de réserver votre jugement pour plus tard et d'explorer le matériel en profondeur. Vos professeurs vous donneront du matériel d'études riches en symboles et en significations. Si vous le rejetez du revers de la main parce qu'il est inconnu ou intimidant, vous imposez à votre apprentissage des entraves inutiles. Pour développer votre sens critique, vous devrez d'abord travailler fort. Vous êtes un peu comme un mineur. Si vous extrayez simplement de l'information, vous extrayez du charbon. C'est utile, mais pas très profitable. Si vous cherchez des concepts et des significations plus profondes dans le charbon, vous augmentez sensiblement vos chances de trouver un diamant (du charbon cristallisé).

Il peut sembler difficile de travailler avec des théories et c'est pourquoi il est bon de se rappeler qu'elles ont été conçues par les ingénieurs intellectuels les plus brillants du monde et sont les meilleurs

\section{Note sur le jargon}

N'ayez pas peur du jargon. II s'agit seulement de mots. Vous utilisez déjà une tonne de termes tirés de jargons quand vous parlez avec vos amis. L'objectif du jargon des études postsecondaires est de permettre aux scientifiques et aux chercheurs de condenser des idées et des concepts complexes en un seul mot ou en une seule phrase. Des termes comme: "sexisme», "bureaucratie», «hégémonie», "classe sociale», « idéologie», « dissonance cognitive», «éthique protestante», "pression par les pairs» et même un mot aussi utilisé que "culture» ont été inventés par des spécialistes des sciences sociales et humaines pour communiquer plus efficacement des idées, et, on ne se le cachera pas, utiliser du jargon correctement vous place dans une classe à part. 
outils de mineurs dont vous disposez. Ne vous attendez pas à ce que les arguments théoriques se lisent comme des articles de magazines: leur objectif n'est pas de divertir, mais d'analyser et d'instruire. $\mathrm{Ne}$ vous laissez pas dissuader par le jargon technique non plus.

Graduellement, plus votre sens critique se développera, plus tout cela semblera moins difficile et le plaisir que vous prendrez à créer du savoir qui a du sens rendra votre travail d'autant plus agréable. Les processus de pensée qui semblent si difficiles en ce moment deviendront pour vous une seconde nature. Vous vous surprendrez à découvrir toutes sortes de choses que seule une intelligence critique peut révéler. La première étape, indispensable, est de suspendre votre jugement et d'explorer. Les résultats en vaudront la peine.

Le développement du sens critique s'apparente plus à un marathon qu'à un sprint de 100 mètres. La persistance et la pratique sont véritablement récompensées. Contrairement à la plupart des habiletés physiques, le sens critique peut s'exercer pendant toute votre vie et l'enrichira infiniment.

\section{Notes}

1. Michael Gilbert, How to Win an Argument, New York, John Wiley, 1996, p. 52. Traduction libre.

2. Benjamin Bloom, Taxonomie des objectifs pédagogiques, Montréal, QC, Presses de l’Université du Québec, 1975-77. 
Page blanche conservée intentionnellement 


\section{Écoute et lecture actives}

«Lire, c'est penser avec la tête d'un autre, au lieu de la sienne.» - Arthur Schopenhauer

Tl est fascinant d'observer les étudiants avant un cours. Quand Iils entrent dans la salle de classe, ils rient, ils placotent, ils discutent de leur plus récente date ou du spectacle de la veille. Ils sont remplis d'énergie et de vitalité, et ont une opinion sur tout. Puis, le professeur arrive, le cours commence et ils saisissent leur ordinateur ou leur stylo, et prennent furieusement en note ce qui n'est ni plus ni moins que diverses informations factuelles. En un clin d'œil, la plupart des étudiants passent d'êtres humains actifs et investis dans leur environnement à des récipients passifs d'un ensemble externe de connaissances. Cette tendance à s'en remettre à l'écoute et à l'apprentissage passifs est le plus gros obstacle au développement du sens critique.

On décrit parfois les cours magistraux comme le transfert des notes de l'orateur au cahier de notes de l'auditeur, sans qu'il y ait compréhension dans l'esprit de l'un ou de l'autre. Bien qu'elle soit stéréotypée, cette description met en évidence que les cours magistraux encouragent l'apprentissage passif, qui mène ensuite à des habitudes d'étude passives. Pour profiter le plus possible d'un cours magistral, vous devez écouter activement et interagir avec le professeur et 
le matériel. Vous devez faire preuve d'initiative. Il s'agit là d'un état d'esprit bien différent de l'écoute et de l'apprentissage passifs !

\section{L'écoute passive}

Quand vous avez une attitude passive pendant un cours :

- vous vous attendez à ce qu'on vous livre l'information toute cuite dans le bec;

- vous voulez que l'information soit structurée de manière à vous aider le plus possible dans vos devoirs et vos examens ;

- vous refusez toute invitation à réfléchir plus profondément à un sujet, à en explorer les ambiguiités, ou à faire quoi que ce soit qui $n^{\prime} e s t$ pas matière à examen;

- vous considérez le sujet clos à la fin du cours, du moins, jusqu'à l'examen.

Être passif ne signifie pas que vous avez été complètement lobotomisé. Vous pouvez toujours faire la différence entre un cours ennuyeux et un cours divertissant. Vous reconnaissez que certains professeurs maîtrisent bien leur matière et sont mieux à même de la transmettre que d'autres. Mais vous n'utilisez pas vos capacités à penser de façon élaborée, celles qui impliquent de comparer, d'analyser, de synthétiser et d'évaluer.

Quand vous êtes en mode passif, il vous est impossible de digérer le matériel et de vous l'approprier. Les faits demeurent dans votre mémoire à court terme et sont oubliés dès que vous avez terminé votre examen final. Le plus tragique dans tout ça? La seule chose que vous retirerez du cours, c'est une note.

\section{L'écoute active}

L'écoute active diffère complètement de l'écoute passive en ce qu'elle implique:

- de porter attention à ce qui se dit, à ce qui est répété et à ce qui est souligné ;

- de chercher des liens entre ce que vous savez déjà et ce qui est expliqué ;

- de vous concentrer sur les idées clés qui vous permettent d'organiser les faits ;

- de prendre part à un dialogue mental avec le professeur ;

- d'identifier sa perspective ou son approche théorique ; 
- d'être conscient des positions qui vont à l'encontre de celle-ci ;

- de garder les idées complexes en tête pour mieux y réfléchir plus tard ;

- de prendre en note les idées qui manquent de clarté.

Les mots qui décrivent le mieux l'écoute active sont: dialogue, réflexion et tension créatrice. $\mathrm{Si}$ vous ne pouvez pas les associer tous les trois à votre façon d'écouter, vous n'écoutez probablement pas activement.

Les premières fois où vous tenterez de pratiquer l'écoute active, votre cerveau, sous-utilisé et peu habitué à ce que vous le sollicitiez autant, réagira probablement tout aussi activement. Vous trouverez l'expérience aussi douloureuse que d'entreprendre un nouveau programme d'exercice physique. Faites donc attention à ne pas exagérer.

Les débutants qui veulent pratiquer efficacement l'écoute active peuvent se concentrer sur un ou deux aspects. La stratégie la plus utile est probablement de déterminer quelles sont les choses que vous ne comprenez pas, de faire preuve d'initiative et d'aller voir votre professeur pour lui demander des clarifications pendant ses heures de bureau ou d'effectuer davantage de recherches de votre côté.

L'écoute active exige toute votre attention. Les distractions, comme un ordinateur allumé, un téléphone intelligent à portée de la main ou même des collègues qui chuchotent, la rendent inatteignable. Il n'est donc pas étonnant qu'elle soit plus facile à atteindre à l'avant ou au milieu, plutôt qu'à l'arrière, de la salle de classe.

\section{La lecture active}

Lire activement requiert également toute votre attention et votre concentration. Ce que vous voulez faire, c'est donner un sens au texte. Le matériel écrit peut avoir plusieurs niveaux de sens. Voici quelquesuns des plus importants :

- Le genre: S'agit-il d'un roman, d'un poème, d'un essai ou de littérature non romanesque?

- Le contexte: À quelle période historique, dans quel cadre physique et dans quel genre de société vivait l'auteur?

- L'auditoire: Le texte était-il destiné aux femmes ou aux hommes, aux adultes ou aux enfants, à un public hautement scolarisé ou aux masses populaires?

- Le message explicite ou implicite : Qu'essaie de dire l'auteur? Qu'insinue-t-il? 
- Les niveaux de sens: Le texte possède-t-il différents niveaux de sens?

- Les sens cachés: Quels sont les symboles ou les codes qu'utilise l'auteur pour transmettre un sens particulier?

- Les sens involontaires : Quels sens les lecteurs qui font preuve de sens critique peuvent-ils donner au texte, même si l'auteur ne les a pas intentionnellement voulus?

- Les sens prêtés : Le texte a-t-il été lu différemment par différents groupes à différents moments?

- La signification scolaire: Quelle est la signification de ce texte dans le cours?

- Le sens personnel: Le texte a-t-il un sens spécial pour vous? Comment vous a-t-il touché?

Vous aurez sûrement remarqué que nous avons gardé le sens personnel pour la fin. Ce n'est pas sans raison. En effet, beaucoup d'étudiants se laissent trop souvent emporter par leur réaction à ce qu'ils lisent. Parce qu'un texte complexe a plusieurs sens possibles, les étudiants pensent souvent, à tort, que leur propre opinion devrait avoir préséance dans l'interprétation de ceux-ci.

La lecture active exige cependant que vous suspendiez votre réaction personnelle, et parfois émotive, à un texte pour mieux en explorer judicieusement, analytiquement et synthétiquement les différents sens. Les premières impressions qu'on se fait des textes, comme des gens, sont souvent trompeuses. Plus vous en apprendrez sur les divers sens possibles d'un texte, plus vous apprécierez le livre, l'essai ou le poème.

\section{La lecture au niveau postsecondaire}

Les textes ne demandent pas tous le même niveau de concentration. Les romans d'espionnage ou d'amour sont destinés à un large marché de consommation passive. Un des objectifs des études postsecondaires est le développement de la lecture active et critique en vous exposant à des formes plus exigeantes de littérature. La réflexion scolaire postsecondaire et la pensée populaire de tous les jours se trouvent aux antipodes l'une de l'autre sur le spectre intellectuel. La dernière se caractérise par des affirmations générales brèves et catégoriques, la première, par des hypothèses objectives, très nuancées et construites avec soin, qui s'appuient sur des preuves et des raisonnements.

Un des grands avantages de la lecture critique ou active est qu'elle vous permet de déconstruire toutes sortes d'écritures, d'exposer les 
stéréotypes et les préjugés, et de développer des positions claires et indépendantes. Elle vous permet également de détecter les techniques de manipulation que les publicitaires utilisent pour vous inciter à acheter leurs produits. Par exemple, les chercheurs qui étudient le marketing ont révélé que l'industrie de la mode use de moyens visant à rendre les femmes insatisfaites de leur corps, ce qui risque d'avoir de graves conséquences, comme l'anorexie et la boulimie.

Vous devez lire la plupart des sites Web de manière critique. En effet, ils font rarement l'objet d'une attention réfléchie ou spécialisée.

Il devrait maintenant être évident que la lecture active diffère de la mémorisation de parties de textes et va au-delà de la description ou des résumés que vous pouvez en faire. L'objectif de la lecture active est de vous obliger à vous creuser la tête et à vous investir plus profondément dans le matériel. Il est préférable de commencer à développer ces habiletés un texte à la fois, mais, quand vous vous serez amélioré, vous devrez impérativement aller au-delà du texte pour créer des réseaux de sens. Vous y parviendrez:

- en établissant des rapports avec des textes similaires;

- en comparant et en évaluant les différentes approches qu'on peut adopter par rapport aux textes ;

- en faisant des liens entre les textes et des articles et des analyses scientifiques à leur sujet.

En plus de prendre du temps, la lecture active peut sembler compliquée au début, mais plus vous vous y exercerez, plus elle deviendra pour vous une seconde nature. À long terme, il s'agit d'une manière beaucoup plus efficace d'apprendre que la mémorisation. Cette dernière repose, en effet, sur la mémoire à court terme et vous aurez oublié la plupart de ce que vous avez lu en moins d'un an.

La lecture active crée des réseaux mentaux de compréhension que vous conserverez pour toujours. Les étudiants qui ont une mémoire photographique, mais qui ne sont pas des lecteurs actifs, jouissent peut-être d'un avantage au départ, mais ils ne pourront jamais concurrencer ceux qui ont développé leurs aptitudes en lecture critique.

Voici quelques trucs, que vous ayez ou non un excellent sens critique :

- Parcourez rapidement le titre, l'introduction, la conclusion, le résumé, les titres de chapitres et l'index pour voir ce qu'ils révèlent sur le livre;

- En particulier dans les manuels de cours, demandez-vous sur quel sujet porte le chapitre. Transformer des affirmations 
en questions est une excellente façon de déterminer ce que l'auteur tente de faire;

- Récitez les idées clés du livre ou de l'article et enregistrez-vous ;

- Quand vous révisez, portez une attention particulière aux idées que vous ne pouvez pas articuler clairement.

En plus des étapes énumérées plus haut, beaucoup d'instructeurs en sens critique ont développé des stratégies de lecture propres à toutes sortes de textes. L'exemple ci-dessous a été développé pour les textes de fiction et non romanesques par Jan Rehner, une professeure primée de l'Université York. Nous l'incluons ici avec sa permission.

\begin{tabular}{|c|c|c|}
\hline & Textes de fiction & Textes non romanesques \\
\hline 1 & $\begin{array}{l}\text { Le titre } \\
\text { À quoi vous fait penser le titre? } \\
\text { Pouvez-vous deviner de quoi parle } \\
\text { l'œuvre? }\end{array}$ & $\begin{array}{l}\text { Le titre } \\
\text { Idem }\end{array}$ \\
\hline 2 & $\begin{array}{l}\text { L'auteur } \\
\text { Avez-vous lu d'autres ouvrages } \\
\text { de cet auteur? Avez-vous des } \\
\text { informations sur sa provenance } \\
\text { ou son contexte, qui indiqueraient } \\
\text { un contenu particulier ou un style } \\
\text { caractéristique? }\end{array}$ & $\begin{array}{l}\text { L'auteur } \\
\text { Idem }\end{array}$ \\
\hline 3 & $\begin{array}{l}\text { Droits d'auteur et lieu de } \\
\text { publication } \\
\text { Que savez-vous sur la période } \\
\text { historique et la culture dans laquelle } \\
\text { cette œuvre a été publiée pour la } \\
\text { première fois? }\end{array}$ & $\begin{array}{l}\text { Droits d'auteur et lieu de publication } \\
\text { Idem }\end{array}$ \\
\hline 4 & $\begin{array}{l}\text { Premiers paragraphes ou premier } \\
\text { chapitre d'un roman } \\
\text { Quelles sont vos impressions des } \\
\text { premiers paragraphes de l'histoire } \\
\text { ou du premier chapitre du roman? } \\
\text { Qu'avez-vous appris jusqu'à } \\
\text { maintenant et que croyez-vous qu'il } \\
\text { arrivera ensuite? }\end{array}$ & $\begin{array}{l}\text { Sous-titres et table des matières } \\
\text { Quels sujets sont au centre du texte de } \\
\text { l'auteur? Est-ce que certains des sous-titres } \\
\text { vous semblent inhabituels? Y a-t-il des sujets } \\
\text { que vous vous attendiez à voir et qui ne s'y } \\
\text { trouvent pas? }\end{array}$ \\
\hline 5 & $\begin{array}{l}\text { Liste de contrôle des personnages } \\
\text { Faites la liste des personnages } \\
\text { importants et du numéro de page où } \\
\text { ils apparaissent pour la première fois. } \\
\text { Notez ce que vous pensez d'abord } \\
\text { des personnages. Plus vous avancez } \\
\text { dans votre lecture, indiquez si vos } \\
\text { premières impressions changent. }\end{array}$ & $\begin{array}{l}\text { Premiers paragraphes ou chapitre } \\
\text { d'introduction } \\
\text { Quelle est la thèse principale de l'auteur ou } \\
\text { l'argument qui guide sa réflexion? Faites la } \\
\text { liste des objectifs déclarés ou sous-entendus } \\
\text { de l'article ou du livre. }\end{array}$ \\
\hline
\end{tabular}




\begin{tabular}{|c|c|c|}
\hline & Textes de fiction & Textes non romanesques \\
\hline 6 & $\begin{array}{l}\text { Points à relever pendant la lecture } \\
\text { Les questions et les catégories } \\
\text { suivantes pourraient vous aider à } \\
\text { réagir au texte. Elles ne seront pas } \\
\text { toutes pertinentes, mais utilisez-en } \\
\text { autant que vous le pouvez. N'oubliez } \\
\text { pas de prendre en note le numéro } \\
\text { de page. } \\
\text { Point de vue. Qui raconte l'histoire? } \\
\text { Est-ce l'un des personnages ou un } \\
\text { narrateur omniscient? Le point } \\
\text { de vue change-t-il à mesure que } \\
\text { I'histoire évolue? } \\
\text { Symboles. Y a-t-il des symboles qui } \\
\text { se répètent ou une certaine imagerie } \\
\text { qui revient? Qu'apportent-ils au sens } \\
\text { global de l'œuvre? } \\
\text { Cadre et ambiance. Où se passe } \\
\text { I'histoire et quelle est l'ambiance } \\
\text { dans laquelle elle a lieu? Quelle } \\
\text { influence cela a-t-il sur vous? } \\
\text { Moments décisifs. Quelles sont } \\
\text { les actions les plus importantes de } \\
\text { l'histoire? Les personnages prennent- } \\
\text { ils des décisions importantes? } \\
\text { Vivent-ils des événements qui leur } \\
\text { donnent une perspective particulière } \\
\text { ou qui les font réagir d'une certaine } \\
\text { façon? Les personnages principaux } \\
\text { changent-ils? } \\
\text { Style. L'auteur a-t-il des mots de } \\
\text { prédilection? Les phrases sont- } \\
\text { elles courtes, longues, simples ou } \\
\text { complexes? L'auteur utilise-t-il le } \\
\text { dialogue de manière efficace? } \\
\text { Préoccupations thématiques. Les } \\
\text { thèmes, dans les ouvrages de fiction, } \\
\text { sont généralement exprimés à l'aide } \\
\text { des personnages, des images et de } \\
\text { l'action. La recherche de thèmes } \\
\text { permet de déterminer sur quoi porte } \\
\text { I'histoire ou le roman. Quels sont } \\
\text { les différents thèmes de l'ouvrage? } \\
\text { Notez au moins deux possibilités. }\end{array}$ & $\begin{array}{l}\text { Points à relever pendant la lecture } \\
\text { Les questions et les catégories suivantes } \\
\text { pourraient vous aider à réagir au texte. } \\
\text { Elles ne seront pas toutes pertinentes, } \\
\text { mais utilisez-en autant que vous le pouvez. } \\
\text { N'oubliez pas de prendre en note le numéro } \\
\text { de page. } \\
\text { Termes et définitions clés. L'auteur } \\
\text { utilise-t-il les italiques pour mettre l'accent } \\
\text { sur certains termes? Les définitions sont- } \\
\text { elles claires? Reformulez-les dans vos } \\
\text { propres mots. Correspondent-elles à vos } \\
\text { définitions? Les mettent-elles en doute? Les } \\
\text { enrichissent-elles? } \\
\text { Cartes, graphiques et illustrations. Si } \\
\text { l'auteur fait usage de supports visuels, } \\
\text { comment ces derniers sont-ils liés à la thèse } \\
\text { principale ou au raisonnement? } \\
\text { Citations et références. L'auteur cite-t-il } \\
\text { ou fait-il référence aux opinions ou aux } \\
\text { publications d'experts ou à d'autres auteurs? } \\
\text { Quel est leur lien avec la thèse principale ou } \\
\text { l'argument de l'auteur? } \\
\text { Exemples et preuves. L'auteur utilise-t-il des } \\
\text { exemples pour clarifier des idées abstraites? } \\
\text { Pouvez-vous illustrer une des idées de } \\
\text { l'auteur par un exemple de votre cru? Y a-t-il } \\
\text { assez de preuves pour appuyer les principaux } \\
\text { arguments? Qu'est-ce que l'auteur aurait pu } \\
\text { dire de plus? } \\
\text { Suppositions et partis pris. L'auteur } \\
\text { affirme-t-il quoi que ce soit qui ne peut être } \\
\text { prouvé? Admet-il avoir certains partis pris } \\
\text { qui pourraient donner une certaine direction } \\
\text { à son raisonnement? } \\
\text { Symboles. Y a-t-il des symboles qui se } \\
\text { répètent ou une certaine imagerie qui revient } \\
\text { pour appuyer les principaux arguments? } \\
\text { Style. L'auteur a-t-il des mots de } \\
\text { prédilection? Les phrases sont-elles courtes, } \\
\text { longues, simples ou complexes? }\end{array}$ \\
\hline
\end{tabular}




\begin{tabular}{|c|c|c|}
\hline & Textes de fiction & Textes non romanesques \\
\hline 7 & $\begin{array}{l}\text { Conclusion } \\
\text { La fin est-elle satisfaisante? Peut-elle } \\
\text { être interprétée de plusieurs façons? }\end{array}$ & $\begin{array}{l}\text { Paragraphes de conclusion ou chapitre de } \\
\text { résumé } \\
\text { La conclusion reprend-elle la thèse } \\
\text { principale? Avez-vous l'impression que } \\
\text { l'argument principal a bien été prouvé? Les } \\
\text { observations finales donnent-elles des idées } \\
\text { qui pourraient être explorées davantage } \\
\text { sur de possibles applications de l'argument } \\
\text { principal? }\end{array}$ \\
\hline 8 & $\begin{array}{l}\text { Impressions d'ensemble et contenu } \\
\text { du cours } \\
\text { Comment cet ouvrage enrichit-il } \\
\text { la connaissance que vous avez des } \\
\text { problématiques abordées dans le } \\
\text { cours? Quelles sont les suppositions } \\
\text { de l'auteur à leur égard? Quel est le } \\
\text { point de vue de l'auteur à ce sujet } \\
\text { comparativement à d'autres auteurs } \\
\text { que vous avez consultés pour ce } \\
\text { cours ou pour d'autres cours? Quelles } \\
\text { suppositions avez-vous vous-même } \\
\text { remises en question à la lecture de } \\
\text { cette histoire ou de ce roman? }\end{array}$ & $\begin{array}{l}\text { Impressions d'ensemble et contenu du } \\
\text { cours } \\
\text { Idem }\end{array}$ \\
\hline
\end{tabular}

\section{Améliorer ses habiletés}

Plus vous améliorerez vos habiletés en lecture, plus votre capacité d'analyse et votre compréhension se raffineront. Voici quelques trucs pour vous guider et vous assurer de progresser:

- Quand vous entamez la lecture de matériel difficile, il est important de le faire de façon logique pour arriver à le comprendre clairement. Plus vous développerez vos habiletés, plus vous pourrez utiliser les côtés créatif, intuitif et imaginatif de votre esprit. C'est ce qu'on appelle souvent la pensée latérale. Les penseurs originaux en font toujours preuve. Mais rappelez-vous que la pensée traditionnelle, logique et séquentielle doit toujours venir en premier. N'essayez pas de courir avant de savoir marcher ;

- Les problèmes les plus intéressants ont toujours de multiples solutions. Dès que vous aurez appris à lire le matériel attentivement et de manière critique, vous voudrez l'aborder à partir de différentes perspectives. Les meilleurs lecteurs prennent très habilement d'autres rôles. Plus ils s'approprient 
la matière, plus ils peuvent jouer le rôle de différents types de lecteurs. Pouvez-vous porter plusieurs chapeaux et vous imaginer comment vous réagiriez à un texte si vous étiez un jeune optimiste, un vieux conservateur, un chef d'entreprise ou un prestataire de l'aide sociale?

- Vous pouvez toujours adopter la perspective qui vous semble la plus utile. Vous pouvez choisir de provoquer, d'être sympathique, ironique, optimiste ou pessimiste. La seule règle à respecter est de demeurer clair et juste dans votre évaluation d'un texte ;

- Les disciplines scolaires offrent certaines des perspectives les plus utiles. Par contre, les penseurs les plus originaux travaillent généralement sur plusieurs plans. Ils sont multidisciplinaires en ce qu'ils utilisent diverses perspectives pour découvrir de nouvelles façons d'appréhender l'information. Un de ces penseurs, Karl Polanyi (1886-1964), a écrit des ouvrages brillants sur la politique et l'économie. Dans son livre, La grande transformation: aux origines politiques et économiques de notre temps, il adopte des perspectives provenant de l'anthropologie, de l'histoire, de la science politique et de l'économie ;

- Le fait que les auteurs sont des scientifiques qui suivent les règles de la recherche scientifique ne signifie pas nécessairement qu'ils n'ont pas de parti pris. En fait, tout le monde a un parti pris, d'une manière ou d'une autre. Une bonne indication que vous devenez un lecteur critique est de pouvoir le détecter ;

- Nous sommes tous des produits de la culture d'où nous provenons et celle-ci a également des partis pris. Si vous les reconnaissez et les remettez en question, votre aptitude à lire de manière critique augmentera de façon spectaculaire. L'étude des préjugés qu'ont les autres cultures envers la vôtre peut être utile à cet égard ;

- Concentrez-vous autant sur ce qu'un auteur ne dit pas que sur ce qu'il dit. Par exemple, jusqu'à tout récemment, la plupart des textes sur le commerce étaient écrits par des hommes pour des hommes. Ils étaient le reflet d'un point de vue limité et laissaient de côté une quantité importante d'informations. Pourquoi les femmes ont-elles été aussi absentes du monde des affaires? De la même façon, pourquoi tant de livres sur le commerce ignorent-ils ou déprécient-ils le rôle des travailleurs ou des syndicats? 
Aussitôt que vous acquerrez de solides aptitudes en écoute et en lecture actives, ce qui se passe dans la salle de classe et au travail deviendra plus intéressant. Encore mieux : votre capacité à contribuer de manière significative augmentera énormément! 


\section{Se documenter sur un sujet}

\footnotetext{
«Dans la vie, rien n'est à craindre, tout est à comprendre. Il est maintenant temps de comprendre davantage pour moins craindre. » - Marie Curie
}

B eaucoup de manuels qui traitent du sens critique passent directement de la lecture active à la rédaction critique. Le problème avec cette approche est qu'elle véhicule le mauvais message. Beaucoup d'étudiants commettent l'erreur de commencer à rédiger trop rapidement après avoir fait leurs lectures et s'étonnent ensuite de souffrir du syndrome de la page blanche. Généralement, souffrir de ce syndrome s'explique par le fait que certaines étapes essentielles ont été omises pendant la recherche.

Les étudiants abordent parfois la rédaction de manière tout à fait irréaliste. Ils se concentrent sur le processus de rédaction au détriment du travail qui doit être fait en amont. En d'autres termes, ils passent le moins de temps possible à se documenter, dans le but avoué de se donner le plus de temps possible pour rédiger. Ils font donc tout le contraire de ce que tout auteur expérimenté ferait.

Les auteurs chevronnés savent que la recherche est l'élément crucial du processus d'écriture. Vous devriez consacrer environ $70 \%$ de votre temps à la recherche et $30 \%$ à la rédaction. Celle-ci sera grandement facilitée si vous avez fait votre recherche comme il faut. En 
fait, certains auteurs disent même que la rédaction coule d'elle-même si la recherche est bien faite. Nous n'irons pas aussi loin, mais cela vous donne une idée de l'importance de la recherche.

\section{Les règles de base de la recherche}

Les lignes directrices suivantes vous aideront à améliorer vos aptitudes en recherche:

- Faites preuve de curiosité. Vos recherches demeureront superficielles si vous ne voulez pas en savoir toujours davantage sur votre sujet. Être curieux vous aidera à formuler les questions ou les problèmes que vous résoudrez dans votre travail ;

- Trouvez un sujet qui vous intéresse. Il est pénible de faire des recherches sur un sujet que vous trouvez ennuyeux. Le but de vous documenter sur un sujet est de satisfaire votre curiosité;

- Utilisez les ressources de la bibliothèque (en personne ou en ligne), les bases de données, les lectures suggérées dans vos cours ainsi qu'Internet pour vous informer sur ce qui existe déjà sur le sujet. Les articles évalués par des pairs (lus de manière critique par des chercheurs avant publication) sont les plus fiables. Les articles de revues scientifiques sont habituellement plus utiles que les livres parce qu'ils sont plus actuels et plus ciblés. Ils peuvent toutefois présenter quelques inconvénients : ils sont souvent hautement spécialisés et destinés à des experts plutôt qu'à des étudiants ; ils se concentrent parfois sur un aspect tellement précis d'un sujet qu'ils ne vous seront d'aucune utilité; ils utilisent parfois un jargon ou des analyses statistiques que vous ne connaissez pas;

- Utilisez les livres et les sources secondaires avec modération. Les étudiants ont tendance à s'en remettre aux livres et, en particulier, aux manuels de classe, pour faire leurs recherches, souvent parce qu'ils en connaissent bien le style et le langage. Souvenez-vous toutefois que ces livres présentent souvent les recherches des autres et qu'ils sont rarement à jour quant aux problématiques les plus actuelles. Ils peuvent, par contre, vous être très utiles pour trouver des sujets de recherche, simplifier des problématiques complexes et fournir des notes et des bibliographies qui vous dirigeront vers des sources intéressantes. Pour être un bon chercheur, vous devez utiliser les 
revues spécialisées qui font état de recherches scientifiques ;

- Ne négligez pas Internet, car il peut, en effet, être très utile pour trouver des faits et des données. Consultez-le davantage pour ce genre de matériel que pour des explications (c'est-àdire des théories et des concepts). Faites preuve de prudence avec les sites Web populaires, comme Wikipédia, pour quoi que ce soit d'autre que vous donner un point de départ pour votre recherche. Les meilleures sources d'information seront toujours les livres et les articles que vous consulterez au moyen du site Web de la bibliothèque de votre établissement d'enseignement. Plus vous utiliserez des sources scientifiques, évaluées par les pairs, plus vous serez assuré que le contenu qui s'y trouve est à la hauteur des standards scientifiques qu'exigent vos travaux postsecondaires;

- Faites attention à la date de publication. Généralement, plus c'est récent, plus c'est pertinent. Les chercheurs s'appuient la plupart du temps sur les recherches précédentes dans leurs propres publications. Vous pouvez donc remonter dans le temps si vous trouvez un sujet qui vaut la peine d'être approfondi. Il n'y a rien de plus frustrant que de travailler avec du matériel qui se révèle dépassé ;

- Soyez à l'affût de débats entre chercheurs. Ceux-ci ont naturellement lieu au sein même de la littérature scientifique et les explorer peut se révéler utile. Dans la plupart des cas, vos professeurs ne s'attendent pas à ce que vous vous attaquiez à un sujet qui n’a jamais été débattu auparavant ;

- Cernez le problème que vous avez l'intention de résoudre ou la question à laquelle vous voulez répondre. La recherche est tout simplement de la résolution organisée de problèmes. Ce fait est tellement essentiel à la compréhension de ce qu'est la recherche que nous ne pourrions le répéter assez souvent. Les problèmes insignifiants ou les questions sans intérêt ne donneront jamais des dissertations ou des réponses intéressantes. Poser des questions purement rhétoriques ne permettra pas de révéler quoi que ce soit de nouveau;

- Faites particulièrement attention quand vous traitez de problématiques controversées. Si vous choisissez d'aborder un sujet hautement émotif ou controversé, vous devrez contrôler vos réactions. Soyez conscient de vos propres partis pris et ayez pour objectif de rédiger de manière équilibrée ; 
- Organisez et analysez minutieusement vos données. Annotez chaque article ou livre qui vous semble utile. Une annotation est un court résumé de quatre ou cinq lignes qui vous rappelle ce que l'auteur a découvert et défendu. N'hésitez pas à ajouter, en une ligne ou deux, vos propres impressions et tout problème et/ou contradiction que vous avez relevés dans l'ouvrage ;

- Composez un synopsis de l'information la plus importante. Un synopsis comprend de l'information provenant de plusieurs sources (livres et articles) et se compose généralement de plusieurs paragraphes. Contrairement aux annotations, il ne s'agit pas d'un résumé de livres ou d'articles. Le synopsis traite l'information dans son ensemble. Il inclut des éléments qui proviennent de votre lecture critique et fait état des détails particuliers ou des exemples qui pourraient vous aider à élaborer votre propre raisonnement. Voici un exemple: «En général, on s'entend, dans la littérature, que [deux paragraphes]. Par contre, Tremblay et Nguyen se distinguent de la masse en suggérant que [un paragraphe]. Dans l'ensemble, la recherche sur le sujet de [un paragraphe] n'est pas satisfaisante. Il est révélateur que personne ne fasse mention de [un paragraphe]. » ;

- Appliquez un raisonnement inductif et un raisonnement déductif aux données. Il n'existe pas de vérité absolue dans les affaires humaines. Les choses ne sont jamais toutes noires ou toutes blanches. Il y a seulement différentes nuances de gris. Pour les comprendre, nous appliquons différents types de raisonnements. Le raisonnement inductif cherche à développer des généralisations à partir des faits. Le raisonnement déductif, quant à lui, utilise des principes, ou des généralisations, pour organiser les faits. En pratique, nous utilisons les deux types simultanément, car l'utilisation de l'un ou de l'autre seulement peut entraîner des difficultés ;

- Établissez une thèse que vous mettrez à l'épreuve. Une thèse peut être conçue comme une solution au problème que vous avez détecté. Elle proviendra de la recherche que vous faites. En effet, ne croyez pas que c'est en regardant dans le vide en espérant un miracle que vous trouverez une thèse ou un sujet ;

- Tirez des conclusions claires et concises. Révisez soigneusement les résultats de vos recherches pour vous assurer qu'ils 


\section{Conseils de recherche}

1. Demandez l'avis de votre professeur pour vous assurer que votre sujet répond à ses exigences. Ne présumez de rien! Ne demandez toutefois pas à votre professeur de vous suggérer un sujet. Le faire est un signe indéniable de passivité.

2. Demandez à votre professeur de vous suggérer des sources possibles pour votre recherche, mais ne vous limitez jamais à celles-ci. Faites preuve d'initiative.

3. Dans plusieurs disciplines scolaires, on publie des résumés de tous les articles parus chaque année dans des revues avec comité de lecture. L'utilisation de ces résumés vous donne un accès direct à l'information la plus utile. Si vous ne savez pas où les trouver en ligne, demandez I'aide des bibliothécaires de votre établissement d'enseignement.

4. Parcourez rapidement les textes afin de déterminer s'ils vous seront utiles. La dernière chose que vous voulez, c'est perdre votre temps.

5. Utilisez des fiches pour prendre des notes et faire des annotations. Utilisez une fiche par référence. De cette façon, vous pourrez réorganiser vos notes et annotations à votre guise.

6. Apprenez à utiliser un logiciel de gestion bibliographique, comme RefWorks. Le logiciel importera les références du Web et appliquera le format désiré à vos notes de bas de page et à votre bibliographie.

7. Lors de la rédaction de vos notes de recherche, utilisez les guillemets pour qu'il soit possible de distinguer vos paraphrases de ce que vous avez copié mot à mot de la source. Si vous ne le faites pas, vous risquez de vous faire accuser de plagiat.

8. Quand vous avez cerné votre sujet ou précisé votre thèse, écrivezle en grosses lettres sur une fiche et mettez-le au-dessus de votre table de travail pour vous aider à rester concentré.

9. Si vous vous rendez compte que vous devez faire du remplissage dans votre dissertation (c'est-à-dire ajouter du bla-bla), vous n'avez pas fait assez de recherches. Retournez en faire.

sont clairs et sans équivoque. L'objectif de la recherche est de concentrer votre analyse sur un point précis. Visez la profondeur au lieu de ratisser large.

Vous pouvez résoudre pratiquement n'importe quel problème de recherche en vous concentrant sur un point précis du sujet. Une erreur que commettent couramment les débutants est de choisir un 
sujet beaucoup trop vaste pour un travail scolaire, lequel fait généralement moins de 2000 mots. Par exemple, un sujet comme le capitalisme mondial est tellement vaste qu'il est impossible de le traiter convenablement dans une dissertation. Par contre, vous concentrer sur l'émergence économique du Japon après la Deuxième Guerre mondiale, par exemple, réglera le problème de l'envergure du sujet.

\section{Deux stratégies de recherche}

Tout le monde peut éprouver des difficultés quand vient le moment d'organiser sa recherche. Vous pouvez avoir une bonne thèse ou un bon sujet, et même une vision claire de ce dont votre travail devrait avoir l'air, puis, au fur et à mesure que vous décidez de l'ordre de votre travail et de ce qui doit aller ici ou là pour être le plus efficace, vous frappez un mur. Certains chercheurs expérimentés parviennent à emboîter les idées les unes dans les autres dans leur tête, mais la plupart des simples mortels que nous sommes ont besoin d'aide pour organiser leurs idées.

Les deux stratégies ci-dessous vous aideront à prendre les idées que vous avez en tête et à les mettre à l'écran ou sur papier.

\section{L'écriture spontanée}

L'écriture spontanée se situe complètement à l'opposé de la rédaction habituelle d'une dissertation. Quand vous composez une dissertation, vous choisissez soigneusement vos affirmations en gardant en tête que vous devrez les défendre. Ce faisant, certaines de vos bonnes idées intuitives se perdent parfois en cours de route. L'écriture spontanée consiste à simplement rédiger ce qui vous vient à l'esprit sur le sujet choisi. C'est une stratégie qui est particulièrement utile quand vous venez tout juste de terminer votre recherche et que vous avez hâte de commencer à rédiger, mais ne savez pas encore comment les morceaux que vous avez amassés forment un tout cohérent.

Les règles de l'écriture spontanée sont simples, mais il est important de s'y tenir. D'abord, soyez bref. N'y passez pas plus de 30 minutes ou vous finirez par rédiger votre dissertation au lieu d'explorer votre documentation. Ensuite, ne pensez pas à ce que vous rédigez. Laissez plutôt votre intuition et votre imagination vous guider. Puis, ne changez rien. Enfin, attendez au lendemain pour relire ce que vous avez écrit et relevez tout ce qui sera utile à la rédaction de la dissertation. 


\section{La carte mentale}

Le cerveau humain est un endroit bizarre, mais merveilleux. Il trie et organise l'information de diverses manières que nous ne comprenons pas tout à fait. La technique de la carte mentale est ce qui se rapproche le plus du fonctionnement du cerveau. Voici comment l'utiliser. Premièrement, déterminez le sujet principal ou la thèse que vous voulez organiser. Mettez-le au centre de la page ou de l'écran.

Deuxièmement, cernez les idées clés du sujet. Ajoutez-les à la carte sous forme de rayons qui partent du sujet principal.

Troisièmement, trouvez les concepts qui sont liés aux idées clés. Vous pouvez en faire des branches ou des réseaux qui proviennent des idées. Si un concept est lié à plus d'une idée clé, utilisez des couleurs différentes ou des lignes pointillées pour illustrer les connexions. N'évitez pas la complexité. Les connexions multiples indiquent généralement des problématiques intéressantes.

Quand vos connexions sont établies, réorganisez le tout en colonnes. Dans la plupart des cas, vous vous rendrez compte que les colonnes sont, à peu de choses près, à l'image de l'organisation

\section{Carte mentale}

\section{Droits}

- Dignité humaine

- Axé sur les règles

- Axé sur le devoir

- Universalité

Vous avez droit à la propriété privée.

Justice

- Équité

- Égalité

- Accès égal aux ressources

Les sociétés capitalistes doivent subvenir aux besoins des faibles.

\section{Utilitarisme}

- Bien public

- Économie de marché

- Principe de plaisir

- Relativité

Les droits des individus n'ont généralement pas d'importance. Les profits sont bons pour tous.

\section{Existentialisme}

- Libre arbitre

- Authenticité

- Intégrité

- Responsabilité personnelle

Les membres des entreprises devraient sonner l'alarme s'ils sont témoins de comportements immoraux. 
naturelle de votre recherche et fournissent le plan de votre dissertation.

Dans l'exemple de carte mentale ci-dessus, le sujet principal, commerce et éthique, se trouve au milieu. Les quatre idées clés, les droits, l'utilitarisme, l'existentialisme et la justice, sont au sommet des différentes colonnes. Les concepts, comme la dignité humaine, le bien public, le libre arbitre et l'équité, sont répertoriés en dessous.

La carte mentale est une technique utile. La prochaine fois que vous serez bloqué, que ce soit à l'école ou au travail, essayez cette approche! 


\section{La résolution de problèmes pratiques à l'école, au travail et dans la vie}

T 'aptitude critique que vous développez au cours de vos études $ـ$ postsecondaires fera de vous un employé intelligent et de grande valeur. Dans un monde parfait, l'aptitude critique et l'aptitude pratique ne feraient qu'une. Malheureusement, il y a souvent un écart entre l'apprentissage théorique et la mise en application, et cet écart nuit à la résolution de problèmes efficace au travail. Beaucoup d'employeurs se plaignent, en effet, du fait que les nouveaux employés possèdent des connaissances à la fine pointe, mais ne sont pas capables de les appliquer aux situations de la vraie vie.

Par goût personnel ou à cause de leur occupation professionnelle, beaucoup de professeurs préfèrent les concepts et les théories. Il ne s'ensuit pas pour autant que leur recherche et leur enseignement ne s'appliquent pas au monde réel. Quoi qu'il en soit, vos professeurs n'ont pas la responsabilité de vous montrer comment obtenir et garder un emploi, ni comment y avoir du succès. Vous devez prendre les choses en main.

Nous nous tournons dans ce chapitre vers le savoir-faire qui vous permettra de réussir sur le marché compétitif du travail. Ce savoirfaire est utile à tous, y compris à vos professeurs! Et il est d'autant 
plus efficace s'il est combiné aux aptitudes en rédaction, en lecture et en écoute, et au sens critique, dont nous avons parlé dans les chapitres précédents.

\section{Pourquoi la résolution de problèmes pratiques est-elle si importante?}

Vos employeurs veulent savoir si vous avez ce qu'il faut pour résoudre des problèmes dans la vraie vie. Bien que la capacité à résoudre des problèmes soit un trait de caractère inné chez les humains, la résolution de problèmes complexes fait toute la différence entre quelqu'un d'extraordinaire et quelqu'un d'ordinaire. Ceux qui peuvent résoudre des problèmes se démarquent de la masse, font l'admiration des autres et sont recherchés par les employeurs.

Nous percevons parfois la résolution de problèmes comme la marque mystérieuse d'une intelligence supérieure ou du génie. Pour paraphraser l'inventeur américain Thomas Edison, le génie est fait d'un pour cent d'inspiration et de $90 \%$ de transpiration. La résolution d'un problème complexe est un travail difficile. Vous devez prendre le temps de le définir, d'en découvrir les causes, d'en explorer les solutions possibles et de choisir la meilleure. Et encore, le travail n'est alors pas terminé puisque vous devez mettre la solution en application et évaluer à quel point elle fonctionne bien.

Vous seriez étonné de constater combien ceux qui sont en position d'autorité ou d'importance prennent des raccourcis ou se basent sur leur intuition pour résoudre des problèmes et obtiennent souvent, comme on s'y attendrait, des résultats mitigés. Il est vrai que l'intuition vous permettra occasionnellement de vous en sortir et vous ne devriez jamais l'ignorer, mais si vous comptez sur celle-ci, sur les conjectures ou, le pire ennemi de tous, sur les fausses suppositions, vos chances de succès sont minces.

Qu'arrive-t-il si vous ne résolvez pas les problèmes correctement? Premièrement, vous perdez un temps précieux et des ressources à faire les choses d'une manière qui n'est pas efficace. Deuxièmement, vous finissez habituellement par adopter des solutions temporaires au lieu de résoudre le problème. Troisièmement, vos efforts deviendront des habitudes et des suppositions autojustifiées sur la façon de procéder, ce qui vous mènera toujours plus loin de la solution optimale.

Dans le monde hautement concurrentiel qui est le nôtre, un tel gaspillage d'énergie n'a pas sa place. Ceux qui tentent de régler les 
problèmes en jouant aux devinettes, en utilisant toujours les mêmes méthodes ou en s'en remettant à de fausses suppositions seront rapidement surpassés par ceux qui sont capables de les cerner et de les régler efficacement. C'est pourquoi beaucoup d'entreprises insistent pour que leurs nouveaux employés soient capables de résoudre des problèmes, mais cette capacité n'est pas utile seulement dans le monde du travail, c'est une compétence de vie. Nous rencontrons tous des problèmes dans la vie. Si nous ne parvenons pas à les définir et à en découvrir la source, notre développement personnel, nos relations interpersonnelles et l'atteinte de nos objectifs sont condamnés à l'échec.

L'apprentissage de la résolution intelligente de problèmes augmentera vos chances de succès dans votre profession et dans votre vie. C'est un des meilleurs investissements que vous puissiez faire et, comparativement à d'autres investissements, il est relativement simple. L'acquisition d'une éducation postsecondaire ou d'une accréditation professionnelle prend des années, mais vous pouvez commencer à utiliser vos nouvelles aptitudes en résolution de problèmes dès que vous aurez terminé la lecture de ce chapitre.

\section{La résolution de problèmes comme processus}

Le plus gros obstacle à une bonne résolution de problèmes est la tendance à se ruer sur une solution. Les problèmes rendent mal à l'aise et, instinctivement, on essaie de trouver immédiatement une solution afin de retrouver sa zone de confort. On s'accroche ensuite à la solution contre vents et marées, la défendant même, toutes griffes dehors, contre de meilleures options.

Prenez, par exemple, la plupart des débats à l'école. Le professeur pose une question (les questions sont, en fait, une forme de problème), les étudiants choisissent leur camp et le dialogue commence. Les positions se raffermissent rapidement et les interlocuteurs deviennent dogmatiques. Les personnalités les plus fortes ou ceux qui sont les plus articulés l'emportent habituellement. Il n'arrive que très rarement, et seulement entre les mains de bons professeurs, que les individus reviennent sur leur position et reconsidèrent leurs affirmations initiales. En outre, peu importe l'appréciation de ces débats par les participants, peu en ressortent avec une nouvelle perspective ou avec une vision approfondie du problème.

La résolution de problèmes à des niveaux plus élevés peut sembler artificielle au début. Elle exige que vous preniez votre temps et 
que vous soyez consciencieux. Elle exige que vous suspendiez toute volonté de vous ruer sur un jugement qui pourrait obscurcir les subtilités du problème et écarter d'emblée certaines solutions. Elle implique que vous accordiez autant d'importance au chemin qu'à la destination. Elle nécessite aussi que vous traciez soigneusement votre itinéraire. Et elle donne des résultats!

Prenez, par exemple, cette critique que les femmes font parfois à l'homme de leur vie, à savoir qu'il préférerait tourner en rond pendant des heures et manquer d'essence que de demander son chemin. Ce scénario est une illustration plutôt simple de l'incapacité à résoudre efficacement un problème, et ses conséquences ne sont pas très graves. S'égarer, perdre son temps et s'énerver les uns contre les autres sont quelques-unes de ces conséquences, mais quelqu'un finira bien par demander le chemin et, en fin de compte, tous arriveront à destination. Espérons seulement que la relation n'en souffrira pas trop!

Dans la vie et au travail, l'incapacité à résoudre les problèmes efficacement peut avoir des conséquences beaucoup plus graves. Par exemple, si un homme et une femme ont des problèmes de communication, ils présumeront peut-être des choses au sujet de l'autre, ce qui finira par détruire leur couple. Si un gestionnaire croit que ses employés ne sont pas productifs parce qu'ils sont paresseux, alors que le problème a plutôt à voir avec le manque d'outils appropriés ou de motivation, il peut miner le moral de tout le monde et même ruiner la compagnie. Si une banque investit les fonds de ses clients dans l'immobilier parce qu'elle suppose que le marché continuera de croître, elle pourrait détruire les plans de retraite de centaines de familles quand les ventes de maisons déclineront. Ces scénarios et d'autres semblables arrivent tous les jours.

Puisqu'une résolution inadéquate des problèmes peut produire des résultats aussi catastrophiques, la meilleure stratégie est d'opter pour un modèle qui permettra d'éviter les pièges habituels. Selon ce modèle, nous recommandons d'élargir d'abord les limites du problème et, ensuite, de les réduire.

Il est en effet très peu judicieux de commencer par circonscrire le problème ou d'essayer de le résoudre rapidement. Vous réduisez ainsi artificiellement le champ de recherche, cachant les causes possibles du problème et limitant votre capacité à mieux le comprendre. C'est seulement après avoir exploré le problème en profondeur et suspendu votre jugement quant à sa solution que vous pourrez commencer à vous concentrer sur ses causes profondes. 
Commencez par grossir le problème : définissez-le et trouvez-en les causes possibles selon autant d'angles que vous le pouvez. Pour ce faire, il se pourrait que vous deviez mener quelques recherches. Si d'autres se sont déjà penchés sur le problème, pourquoi ne pas tirer profit de leur travail ? Il vaut la peine d'explorer la littérature théorique. Comme nous l'avons indiqué dans les chapitres précédents, les concepts et les théories sont les outils de résolution de problèmes les plus sophistiqués. Y en a-t-il qui s'appliquent directement à votre problème? Pourriez-vous adapter un cadre théorique pour explorer celui-ci plus en profondeur?

Il peut être efficace d'explorer un problème en groupe ou de faire un remue-méninges à son sujet. Cette façon de faire permet d'obtenir le plus d'opinions possible. Ceux qui sont les plus aptes à résoudre les problèmes ne s'enchaînent pas à leur bureau pour travailler isolément des autres; ils vont à la recherche de la contribution des autres et récoltent le plus d'idées possible. Idéalement, les membres de votre équipe auront différents parcours professionnels ou personnels, et fourniront des perspectives théoriques variées ainsi que des orientations personnelles, qui auront une incidence sur le problème. Si vous devez travailler seul, la meilleure stratégie est de demeurer le plus ouvert possible au problème et à ses causes, et d'explorer le plus de points de vue différents que vous le pouvez.

Un obstacle majeur à la résolution efficace de problèmes, et à la prise de décisions en général, est de ne pas faire appel à ceux dont la contribution a le plus de valeur. C'est une erreur trop fréquente commise par les dirigeants d'entreprise, qui omettent de consulter leurs collègues, comme les gestionnaires de départements et le personnel de première ligne, qui sont souvent ceux qui ont le plus de détails sur le problème. Parfois, les employés les moins influents de l'entreprise, comme ceux qui traitent les plaintes des clients quotidiennement, peuvent faire les meilleures suggestions. Personne n'osera affirmer qu'une entreprise doit être démocratique, mais le gestionnaire qui ne demande pas l'avis de tout employé qui pourrait apporter sa contribution à la résolution d'un problème particulier n'est probablement pas très habile à résoudre des problèmes.

$\mathrm{Au}$ tout début du processus de résolution de problèmes, la fonction est beaucoup plus importante que la forme. Autrement dit, ne vous en remettez pas uniquement aux règles; allez de l'avant et faites le travail. Encouragez les remue-méninges et les positions différentes au lieu de trop vous concentrer sur les règles. Par exemple, 
vous pouvez utiliser les diagrammes, comme la carte mentale dont nous avons parlé dans le chapitre précédent. Rappelez-vous toutefois qu'il s'agit seulement d'un outil pour vous aider à visualiser le problème. Une fois que vous avez accumulé assez de données, il est très important de les analyser avec rigueur. Plus vous avancerez vers la résolution de votre problème, plus vous devrez être stratégique. Vous circonscrirez l'information avec de plus en plus de précision.

D'aucuns croient que leur travail est terminé dès qu'ils trouvent une solution. Pas du tout! Ils doivent décider comment la mettre en œuvre et qui le fera. Et ce n'est toujours pas fini : la résolution de problèmes est une activité continue et sans fin. Dans plusieurs cas, vous ne saurez pas si un problème est résolu tant que vous n'aurez pas la preuve tangible du succès de la solution. De plus, la vraie vie est compliquée. Vous avez peut-être résolu le problème initial, mais vous en avez créé de nouveaux au passage. Le succès d'un côté peut mener à des accrocs de l'autre. Aussi devrez-vous évaluer les impacts positifs et négatifs de la solution choisie. Finalement, le monde est en constante évolution et vous devrez réévaluer la nature du problème et la solution, parfois plus d'une fois.

La résolution efficace de problèmes est ardue, mais elle n'est pas mystérieuse. Si vous suivez les sept étapes ci-dessous, vous pourrez efficacement résoudre pratiquement n'importe quel problème.

\section{Étape 1 : définir le problème}

Définir le problème devrait être un exercice plutôt simple, n'est-ce pas? Eh bien, non! Vous seriez étonné du nombre de façons possibles de décrire un problème. C'est pourquoi on doit le faire clairement. Ceux qui sont touchés par les solutions possibles doivent également être d'accord avec sa définition. C'est un processus qui suppose :

- d'énoncer le problème de manière objective et factuelle ;

- d'exclure les ambiguïtés qui pourraient entraîner des culsde-sac;

- de définir le problème de manière à rendre possible sa résolution.

Examinez l'affirmation du problème suivant :

Les étudiants de première année ne sont pas prêts à faire des études postsecondaires parce qu'ils n'ont pas appris à lire et à écrire comme il faut au secondaire. 
Les professeurs font souvent ce genre de déclaration. Celle qui nous intéresse ici n'apporte malheureusement rien d'utile parce qu'elle comporte des suppositions implicites qui risquent de nuire à la résolution du problème. Les suppositions sont les suivantes:

- Le problème trouve sa source au secondaire. Cette affirmation suggère d'emblée une cause, excluant ainsi toutes les autres causes possibles ;

- On doit mieux enseigner aux étudiants au secondaire. Cette supposition suggère une solution : on doit modifier l'enseignement au secondaire.

Et si le secondaire n'était pas la seule cause du problème, voire sa principale cause? Selon vous, y a-t-il d'autres causes possibles? L'énoncé ne laisse-t-il pas entendre que vos professeurs sont déchargés de toute responsabilité de vous aider à développer vos aptitudes en lecture et en rédaction?

L'énoncé n'apporte rien d'utile parce que sa solution sous-entendue n'avancera en rien ceux qui ont cerné le problème. Il ne guide aucunement les professeurs qui voudraient aider leurs étudiants à améliorer leurs aptitudes. L'énoncé d'un problème doit être pertinent pour être utile.

Vous pourriez étoffer l'énoncé en y incorporant un objectif. Cette façon de faire aide tout le monde à se concentrer sur les résultats. Elle comporte cependant quelques possibles inconvénients : l'objectif pourrait être irréaliste ou trop restrictif, ou encore il pourrait bloquer d'autres avenues.

Voici une façon de reformuler l'énoncé du problème pour y inclure les objectifs souhaités :

$40 \%$ des étudiants de première année échouent ou abandonnent au moins un cours. D'ici deux ans, nous réduirons ce pourcentage à $30 \%$.

Vous remarquerez que nous avons introduit une échéance pour atteindre notre objectif. Encore une fois, cela permet à tout le monde de prendre le problème au sérieux et incite à le résoudre dans des délais réalistes. Il va sans dire qu'un échéancier strict ne fonctionne pas nécessairement pour tous les aspects d'un problème. Dans ce cas, vous pouvez distinguer les objectifs à long terme, qui peuvent avoir des échéances flexibles, des étapes à court terme, qui sont mesurables et qui mènent à l'objectif à long terme. 


\section{Étape 2: découvrir les causes}

Ce n'est pas un hasard si nous utilisons ici le terme "découvrir». Trouver la cause d'un problème s'apparente davantage à une exploration qu'à un exercice de logique. À ce stade, la logique n'est pas votre alliée puisqu'elle peut vous empêcher de garder l'esprit ouvert. La logique exige que vous réduisiez le nombre de causes possibles, alors que votre objectif, pour l'instant, est d'être le plus inclusif possible.

D'abord, faites la liste de toutes les causes possibles du problème, sans rien omettre, peu importe à quel point elles semblent tirées par les cheveux. Pour construire un diagramme de cet exercice de remueméninges, vous pouvez utiliser la méthode de la carte mentale décrite au chapitre précédent. Notez simplement toutes les idées qui vous viennent à l'esprit. Déterminez ensuite lesquelles sont liées et dessinez les liens qui les unissent.

Ensuite, organisez les idées de manière un peu plus systématique. Vous pourriez utiliser un diagramme en arêtes de poisson pour vous aider à séparer les causes secondaires des causes principales. Un diagramme en arêtes de poisson ressemble au squelette d'un poisson. Vous pouvez ajouter autant d'arêtes que vous le voulez le long de la colonne vertébrale. Celles-ci représentent vos causes principales. Les arêtes moins importantes - les causes secondaires - sont accrochées aux causes principales. Il serait judicieux de diviser les arêtes en catégories pour qu'il soit plus simple de les organiser.

Par exemple, dans le cas des étudiants en première année d'études postsecondaires, un diagramme en arêtes de poisson pourrait avoir l'air de ceci. La tête (le cercle) est le résultat (beaucoup d'étudiants qui échouent ou qui abandonnent leurs cours). Les grosses arêtes (les boîtes rectangulaires) sont les causes majeures ou principales, alors que les arêtes plus petites sont des causes secondaires ou moins directes. Ces dernières peuvent comprendre :

- un sens critique sous-développé ;

- des styles d'apprentissage différents de ceux acquis au secondaire ;

- de mauvaises aptitudes en gestion du temps et en organisation ;

- le besoin de travailler à temps partiel ou à temps plein.

Vous n'avez pas terminé! À ce stade, vous devez déterminer les causes principales. Pour ce faire, vous devez faire preuve de jugement. Bien que nous vous ayons conseillé de réserver votre jugement et de ne pas sauter aux conclusions trop rapidement, vous devriez 


\section{Diagramme en arêtes de poisson}

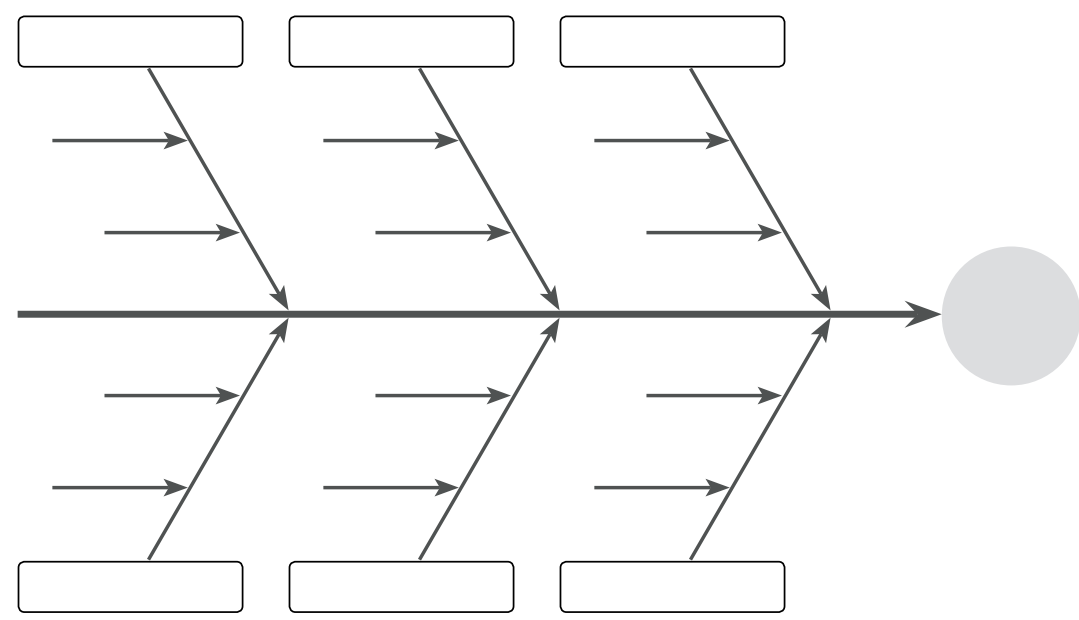

maintenant avoir colligé assez d'informations pour évaluer les différentes options.

La façon dont vous aborderez cette partie du travail aura d'importantes répercussions sur tout ce qui viendra par la suite. Par exemple, vous pourriez décider que la raison principale pour laquelle les étudiants ont des difficultés est qu'ils doivent travailler à temps partiel ou à temps plein pour payer leurs études. Il pourrait être ici intéressant d'appliquer un cadre théorique marxiste, qui suggérerait que le problème principal, ou la source du conflit, est l'accès inéquitable aux ressources économiques dans une société capitaliste. Jusqu'à ce que le problème économique de base soit réglé, la plupart des solutions seront toujours temporaires. Par contre, si vous concluez que les étudiants de première année ont des difficultés à l'école parce que l'établissement postsecondaire n'arrive pas à s'adapter à leurs styles d'apprentissage, rien de tout cela ne s'appliquera et vous irez dans une direction complètement différente.

Certaines causes principales peuvent être intimement liées à des causes secondaires. Par exemple, les étudiants dont le travail rémunéré nuit à leurs études auraient avantage à acquérir de meilleures aptitudes en gestion du temps. Cette affirmation n'a toutefois pas la même 
signification que celle qui déclarerait que les étudiants échouent parce qu'ils ne gèrent pas bien leur temps. Étant donné que beaucoup d'étudiants travaillent à temps plein pendant qu'ils étudient, on peut, en effet, supposer qu'ils ont d'excellentes aptitudes en gestion du temps. L'adoption d'une perspective théorique marxiste peut vous permettre de reconnaître les partis pris inhérents aux approches qui blâment les victimes pour leur persécution.

Comment distinguer les causes principales des causes secondaires? Pour trouver les causes principales, ou profondes, vous devez soumettre chacune d'elles à un feu nourri de questions qui viseront à déterminer «pourquoi ». Autrement dit, continuer de vous demander pourquoi jusqu'à ce que vous ne trouviez plus de réponse. Reprenons l'exemple des étudiants qui échouent en première année d'études postsecondaires.

Pourquoi autant d'étudiants de première année échouent-ils ou abandonnent-ils? Réponse: parce qu'ils n'ont pas les compétences essentielles pour réussir à l'école. Pourquoi n'ont-ils pas ces compétences? Réponse: parce que leurs cours postsecondaires ne visent pas vraiment à les leur inculquer et parce qu'ils ne les développent pas eux-mêmes.

Pourquoi ne développent-ils pas ces compétences? Ils ne gèrent pas leur temps efficacement, que ce soit pour profiter des ressources disponibles ou pour travailler sur leur développement. Pourquoi ne gèrent-ils pas leur temps? Il pourrait y avoir plus d'une cause. Comme nous le suggérons dans ce livre, beaucoup d'étudiants ne se rendent pas compte que les compétences dont ils ont besoin pour réussir à l'école sont aussi celles dont ils auront besoin pour réussir dans le monde professionnel. Une autre cause pourrait être qu'ils travaillent tellement $\mathrm{qu}^{\prime} \mathrm{ils}$ manquent de temps pour se concentrer sur leurs études et en ont encore moins à consacrer au développement des compétences essentielles à leur succès scolaire.

Pourquoi passent-ils tant de temps à travailler ? Est-ce parce qu'ils proviennent de familles à faible revenu et doivent donc financer euxmêmes leurs études ? Ou est-ce parce que les emplois d'été ou à temps partiel ne paient pas assez et qu'ils doivent compenser en travaillant plus d'heures pour accumuler assez d'argent? Ou est-ce parce qu'ils veulent s'acheter certaines choses: voitures, vêtements, téléphones intelligents? Ou est-ce parce que les droits de scolarité sont élevés?

Bien sûr, il n'est pas nécessaire que vous soyez d'accord avec l'une ou l'autre de ces raisons possibles. Notre but ici est de vous montrer 


\section{Le lien entre les théories et la mise en œuvre de la résolution de problèmes}

Les théories permettent de se demander "pourquoi» de manière organisée et de trouver ainsi la source des problèmes. Elles aident à éviter les culs-de-sac analytiques et vous motivent à mettre de côté toute supposition que vous pourriez faire d'emblée.

Résoudre des problèmes sans utiliser de théories s'apparente grandement à essayer de réinventer la roue. C'est un processus d'essais et erreurs, qui donne des résultats mitigés. C'est pourquoi il est si important de vous tenir au courant des développements théoriques dans votre domaine de prédilection.

Si vous vous rappelez que les théories sont les outils de résolution de problèmes les plus sophistiqués et les plus éprouvés, vous vous épargnerez beaucoup de travail inutile.

comment vous pouvez découvrir la cause profonde du problème en demandant «pourquoi ». Si les étudiants doivent travailler de nombreuses heures pour payer leurs études, ils n'auront pas beaucoup de temps pour se développer sur le plan scolaire. Même si on met des ressources à leur disposition sous la forme d'ateliers d'écriture, de séminaires sur le sens critique et de counseling personnel, ils n'auront peut-être pas le temps ou l'énergie d'en profiter. Les bourses, les cours en ligne, la possibilité d'étudier à temps partiel et d'autres solutions du même genre pourraient bien avoir une plus grande incidence sur leur réussite que des mesures bien intentionnées qui ne s'attaquent pas à la source du problème.

\section{Étape 3 : dresser la liste des solutions}

Une fois que vous aurez cerné le problème et en aurez trouvé les causes principales, vous aurez perdu votre temps si vous ne suggérez pas une solution appropriée. Les solutions idéales ne tombent pas du ciel. Elles dépendent des habitudes, des coutumes, des procédures et des règles organisationnelles, sans parler des restrictions évidentes, comme le temps et l'argent.

Quand vous étiez à l'étape de la description du problème et que vous en cherchiez les causes, vous aviez très peu de contraintes, outre l'utilisation du gros bon sens. Dès que vous commencerez à 
suggérer des solutions dans toute organisation ou relation, vous serez assailli de toutes parts par ceux qui ne seront pas d'accord avec vous. Il s'agit là d'une des raisons pour lesquelles il est important d'appliquer de nouveau le processus que vous avez utilisé quand vous cherchiez les causes du problème. En d'autres termes, vous devez chercher à élargir vos horizons avant de les réduire. Parfois, le chemin qui semble le moins praticable se révèle le meilleur si vous le considérez sérieusement comme une possibilité. Ce n'est pas parce que quelque chose semble étranger ou inhabituel qu'il ne s'agira pas d'une solution viable.

Commencez donc par dresser une liste, plus longue que courte, de solutions. Utilisez la technique du remue-méninges pour la créer et, pour le premier jet, incluez tout ce qui vous vient en tête. Voici quelques conseils pour faire un remue-méninges qui vise à trouver des solutions :

- Ne portez pas de jugement sur quoi que ce soit. Beaucoup de scientifiques ont d'abord pensé que le réchauffement de la planète n'avait pas de sens ;

- Sortez des sentiers battus ;

- Soyez prêt à aller au-delà de vos opinions personnelles, de vos habitudes et de votre expérience;

- Examinez en profondeur toutes les possibilités, même si elles semblent ridicules.

Vous devrez finir par raccourcir votre liste. Mais, avant d'éliminer une solution, demandez-vous si elle a des chances de bien fonctionner si vous la combinez à une autre. Parfois, des solutions qui fonctionneraient mal seules conviendront tout à fait si vous les joignez à d'autres.

\section{Étape 4 : faire un choix}

Choisir la meilleure solution requiert que vous réduisiez les possibilités. Ce faisant, vous devez adopter une approche rigoureuse, en prenant en considération les réalités pratiques auxquelles vous et votre organisation faites face. La meilleure façon de faire un choix est d'établir des critères de sélection, d'en déterminer l'importance et de mettre en place un système de points pour mesurer les différentes solutions.

Les grandes organisations publiques et privées utilisent une approche similaire, entre autres quand elles embauchent du personnel. Leur département des ressources humaines dresse une liste de 
critères à utiliser lors des entrevues des candidats. Cela permet de contrecarrer l'instinct naturel de trop s'attarder à la personnalité et à la prestation d'un candidat plutôt qu'à ses réelles forces. Si on pose des questions qui s'appuient strictement sur les critères d'embauche, on élimine une bonne part de partis pris et de favoritisme.

De la même façon, si vous déterminez au moins cinq critères qui vous permettront d'évaluer les solutions, vous augmentez vos chances de choisir la meilleure. De tels critères n'ont pas besoin d'être compliqués :

- coût ;

- simplicité de la mise en œuvre ;

- chances de réussite ;

- à quel point elle élimine le problème ;

- l'adhésion potentielle des membres de l'organisation.

Tous ces critères doivent également être pondérés. Vous pourriez penser que l'élimination du problème est le critère le plus important, mais ce n'est peut-être pas le cas. Si l'élimination du problème vous met à dos plusieurs membres de l'organisation ou ses clients, la résolution du problème ne se trouvera pas au sommet de la liste. Inversement, si l'adhésion n'est pas une considération importante, elle aura moins de poids.

Voici une liste de critères que pourrait utiliser un établissement d'enseignement postsecondaire pour tenter de réduire le nombre d'étudiants de première année qui ne réussissent pas très bien dans leurs études. Dans cet exemple, les différents critères ont été pondérés et leur importance est indiquée par un pourcentage :

- coût $(25 \%)$;

- simplicité de la mise en œuvre (35\%) ;

- chances de réussite (15\%);

- élimination du problème $(25 \%)$.

Pour toute compagnie motivée par les demandes du marché, les chances de réussite et l'élimination du problème auraient plus de poids. Dans un établissement d'enseignement public, par contre, d'autres facteurs peuvent avoir plus d'importance.

Il est essentiel d'avoir des critères, de les évaluer et de les classer en ordre d'importance. Si vous négligez cette étape, la solution que vous adopterez ne sera peut-être pas la meilleure pour l'organisation. Les agences de rencontre utilisent tout le temps cette méthode pour répondre à un genre précis de problème: trouver le meilleur parti pour quelqu'un. Pour ce faire, ils demandent aux clients de classer les 
critères d'évaluation du partenaire idéal en ordre d'importance. Une personne pourrait utiliser les critères suivants :

- attrait physique $(25 \%)$;

- intelligence (20\%);

- sens de l'humour (15\%);

- appréciation de la nature (15\%);

- compatibilité $(20 \%)$;

- revenu (5\%).

Ces pourcentages varieront d'un client à l'autre. Quel serait votre classement pour trouver votre partenaire idéal ? Croyez-vous que dresser au préalable une liste de critères pertinents vous aiderait à faire un meilleur choix et à éviter des problèmes de compatibilité plus tard?

Comme nous en parlons dans le chapitre 9, les employeurs utilisent le même genre de classement quand ils font passer des entrevues à des candidats à un emploi. Par exemple, un employeur pourrait utiliser cette pondération pour un emploi précis :

- habiletés en communication ( $30 \%)$;

- résolution de problèmes $(\mathbf{1 0} \%)$;

- travail d'équipe (35\%);

- flexibilité ( $25 \%)$.

\section{Étape 5 : mise en œuvre}

Passer de la réflexion stratégique à l'action comporte trois étapes qui sont très souvent laissées de côté, même dans les grandes entreprises où les actions inefficaces peuvent avoir des conséquences désastreuses. Pour mettre en œuvre votre solution, vous devez faire un plan d'action ou un échéancier, qui inclut les trois étapes suivantes:

1. diviser la solution en phases précises ;

2. choisir une échéance pour chaque phase ;

3. décider qui sera responsable de s'assurer qu'elles sont terminées.

Jusqu'à ce qu'un plan d'action soit complètement exécuté, c'est tout ce qu'il est: un plan. Ce n'est pas un document légal dont les obligations et les conditions doivent absolument être respectées. C'est pourquoi quiconque élabore un plan d'action aurait probablement intérêt à établir, en parallèle, un plan en cas d'imprévus, dont l'objectif sera de prévoir les problèmes possibles lors de la mise en œuvre et de suggérer des façons de les résoudre. 
Vous pouvez élaborer un plan en cas d'imprévus en déterminant ce qui peut mal tourner à chaque stade principal du plan d'action. Il ne sera pas aussi détaillé que ce dernier, mais il ne doit pas non plus venir après coup. De plus, ceux qui sont responsables de la mise en œuvre du plan d'action devraient le connaître pour les raisons suivantes :

- Ils sauront exactement quoi faire si le plan prend du retard ou si de nouveaux éléments provoquent des changements ;

- Ils seront en mesure d'adapter le plan pour faciliter la transition au plan en cas d'imprévus ;

- Ils auront davantage confiance en eux quand ils feront face à des événements imprévus s'ils savent qu'il existe des options ;

- Dans l'ensemble, ils se sentiront plus responsables de la mise en œuvre.

Les gestionnaires autoritaires ou directifs, qui veulent tout contrôler et qui se croient tout-puissants, commettent parfois l'erreur de garder secret le plan en cas d'imprévus. Ils croient que les employés l'utiliseront comme excuse pour s'éloigner du plan d'action principal. La majorité des théories progressistes en gestion dénoncent ce genre d'approche négative, surtout dans le cas des professionnels dont le rôle dans le succès de l'entreprise équivaut souvent à celui de leurs supérieurs.

\section{Étape 6: points de repère et évaluation}

Comment savoir si votre plan d'action et votre plan en cas d'imprévus répondent aux besoins? Comment saurez-vous que vous avez réussi à éliminer le problème ? Beaucoup d'organisations élaborent et mettent en œuvre des plans compliqués, mais hésitent à évaluer leur progrès. Voici comment éviter de faire cette erreur :

- Établissez des points de repère pour mesurer votre progrès ;

- Accumulez des données qui indiqueront si votre solution est un succès ou un échec ;

- Choisissez les critères qui vous permettront d'évaluer le résultat global.

\section{Étape 7 : rester vigilant}

Une fois que le plan d'action a réussi et que le but est atteint, il vous reste une dernière chose à faire: mettre en place des directives pour vous assurer que le problème ne se reproduise pas. L'ennui avec les 
problèmes, c'est qu'ils sont comme des mauvaises habitudes: vous pouvez vous en débarrasser, mais dès que vous baissez la garde, ils ont tendance à réapparaître. Étant donné que la résolution des problèmes nécessite beaucoup de travail, s'assurer qu'ils ne se reproduisent pas en vaut grandement la peine.

Par exemple, disons que vous êtes en couple avec quelqu'un, mais que la relation bat de l'aile. Après avoir déterminé le problème (ou les problèmes), vous pouvez essayer de le résoudre à l'aide d'une solution conjointe ou vous pouvez prendre vos jambes à votre cou et trouver quelqu'un d'autre. Cependant, si vous ne faites pas attention à ne pas reproduire la première relation, celle qui a fini par mal tourner, le problème réapparaîtra probablement avec la nouvelle personne.

\section{La résolution de problèmes comme compétence de vie}

Nous nous sommes concentrés, dans ce chapitre, sur la résolution de problèmes au sein d'organisations, mais elle n'est pas le propre de la vie professionnelle. La résolution de problèmes s'applique à tous les aspects de la vie, en plus d'être essentielle à l'établissement et à l'atteinte d'objectifs de vie. La construction de bonnes relations et la dissolution de confrontations potentielles ou réelles avec les autres ont une grande importance dans la vie. Trouver la cause profonde de conflits personnels est un premier pas vers la création d'une solution mutuellement acceptable.

Il serait donc sage d'améliorer vos habiletés en résolution de problèmes avant de terminer vos études, si ce n'est que parce que vos employeurs potentiels voudront la preuve que vous possédez ces compétences essentielles. Vous ne serez peut-être pas passé maître dans l'art de résoudre des problèmes, mais vous aurez certainement des expériences de vie qui démontreront vos capacités à le faire ainsi que votre potentiel en tant qu'employé. Lors d'une entrevue, on demande souvent aux candidats de donner un exemple de problème qu'ils ont eu au travail, à l'école ou avec quelqu'un, et de dire comment ils l'ont réglé. Si vous êtes en mesure de démontrer aux employeurs potentiels que vous avez utilisé certaines des sept étapes ci-dessus pour aborder des problèmes, vous deviendrez soudainement un candidat beaucoup plus intéressant. 


\title{
Faire preuve de créativité en résolution de problèmes
}

\author{
«Faites confiance à cette petite voix \\ tranquille qui dit "Ça peut marcher et il \\ faut essayer." » - Diane Mariechild
}

'importe quel artiste qui se respecte vous le dira : la créativité est
une récompense en soi. Elle égaie la vie et fait qu'elle vaut la peine d'être vécue. La pensée créative est aussi un attribut hautement prisé, en particulier dans le monde complexe où nous vivons. Notre rythme de vie effréné et le rapport obsessionnel que nous avons avec la technologie renforcent le raisonnement expérientiel et réactif, lequel nous mène à " prendre l'action pour la pensée et le divertissement passif pour la participation créative ${ }^{1} \gg$. Dans notre société surstimulée, la pensée créative est en déclin. Ceux qui en font preuve se font de plus en plus rares.

Certains croient que la créativité est génétique, que si vous n'êtes pas né créatif, vous ne le serez jamais. On entend souvent les gens dire "Je ne suis pas très créatif», comme si cette lacune était irréparable. Dans les faits, tout le monde est créatif. Avez-vous déjà remarqué comment les enfants utilisent leur imagination quand ils jouent? L'imagination rend tout possible pour eux. Ils ont une capacité remarquable à forger des alliances magiques avec leur environnement. Vous réapproprier cette caractéristique d'enfant peut contribuer à votre bonheur et à votre succès dans la vie. 
Par contre, ceux qui affirment que la créativité ne s'enseigne pas n'ont pas complètement tort. Être créatif relève plutôt de la libération de quelque chose qui est demeuré bloqué pendant toutes ces années où vous avez appris d'autres précieuses formes de savoir. Accroître votre créativité exige de vous débarrasser d'habitudes trop contraignantes.

La bonne nouvelle, c'est que vous pouvez apprendre à le faire grâce à certaines techniques. Et, ironiquement, vous pouvez acquérir ces techniques de manière tout à fait logique et méthodique.

\section{Le lien avec la résolution de problèmes}

La plupart du temps, la résolution de problèmes s'appuie sur la logique et l'expérience. Elle considère les faits concrets et utilise des outils conceptuels pour organiser l'information. La créativité met à contribution un ensemble différent de muscles mentaux. Forme fluide et flexible de raisonnement réflexif, elle se caractérise par une approche descendante. Le cerveau humain crée sa propre réalité au lieu de seulement y réagir. Mais l'esprit n'existe pas isolément et la créativité ne se produit pas en vase clos.

Vous pouvez trouver beaucoup de solutions à des problèmes en suivant les étapes décrites au chapitre précédent, mais certains exemples ne se conformeront pas à un modèle ou à une routine et nécessiteront que vous adoptiez une approche plus créative. Le cas échéant, il est important de penser avec spontanéité et sans inhibition.

La pensée créative est amusante. Elle est foncièrement enjouée. C'est pourquoi les enfants ont généralement plus de facilité que les adultes à être créatifs. Ils prennent le temps de jouer mentalement et sont hautement motivés à être créatifs. En tant qu'adultes, nous avons tort de mettre cette aptitude d'enfant de côté, car les problèmes les plus difficiles et les plus importants dans la vie, à l'école et au travail nécessitent parfois de la créativité. En adoptant une approche trop rationnelle, nous limitons notre capacité à trouver des solutions.

La plupart d'entre nous développeront une certaine facilité à résoudre des problèmes de manière rationnelle. Beaucoup de diplômés, mais certainement pas tous, possèdent cette habileté. Ce qui différencie ceux qui peuvent résoudre les problèmes de façon créative de ceux qui peuvent le faire de manière typique, c'est leur capacité à affronter et à résoudre les problèmes qui déconcertent la plupart des gens.

Voici un petit test pour votre pensée créative. Faites tomber une pièce de dix cents dans une bouteille de vin vide et remettez le 


\section{Ce que les scientifiques ont appris}

Les scientifiques suggèrent que la résolution de problèmes conventionnelle a principalement lieu du côté gauche du cerveau, alors que le côté droit aborde la réalité de manière un peu plus divergente ou créative. Tous les enfants sont des «machines à créativité», mais plus ils vieillissent, moins ils pensent avec le côté droit de leur cerveau, lequel devient plus difficile d'accès avec le temps, le côté gauche du cerveau prenant alors complètement le dessus.

L'intelligence conventionnelle n'équivaut pas à l'intelligence créative. En fait, si le côté gauche du cerveau est endommagé, de plus grands pouvoirs de création sont libérés. Ce phénomène est causé par le fait que la logique et la mémoire perdent le contrôle de certaines fonctions du cerveau. La capacité à «lâcher prise» est caractéristique de la créativité.

La pensée surspécialisée peut également nuire à la créativité. Les experts qui adhèrent de manière trop stricte à des modèles disciplinaires finissent souvent par perdre leur flexibilité conceptuelle. Si leur approche ne fonctionne pas, ils se retrouvent souvent dans un «culde-sac mental ». C'est pourquoi l'utilisation d'une approche multidisciplinaire ou interdisciplinaire est intrinsèquement plus créative que celle qui fait appel au modèle d'une seule discipline.

Idéalement, la créativité sollicite les deux côtés du cerveau. Les idées les plus utiles combinent pensée imaginative et savoir théorique disciplinaire. Par exemple, la théorie de la relativité d'Albert Einstein (1879-1955), qui est probablement la percée créative la plus connue, est fondée sur la connaissance experte qu'il avait de la physique et des mathématiques conventionnelles ${ }^{2}$.

bouchon. Votre tâche est de récupérer la pièce de dix cents sans tirer sur le bouchon ni briser la bouteille. La solution implique que vous réfléchissiez en dehors de vos sentiers battus mentaux.

Avez-vous pensé à pousser le bouchon dans la bouteille ? Bravo !

L'objectif de ce genre de casse-tête est de démontrer à quel point notre esprit est structuré de manière rigide et de nous habituer à sortir des sentiers battus. 


\section{La rigidité de l'esprit}

Il est nécessaire d'éliminer certains obstacles majeurs pour être en mesure de penser de manière créative. Si ceux-ci ne sont pas éradiqués, vous ne retiendrez aucune technique de pensée créative. Vous pouvez découvrir vous-même certains de ces obstacles en réfléchissant au fait que les enfants sont plus créatifs que les adultes. Vous en trouverez cinq ci-dessous.

\section{La suffisance}

Être suffisant signifie être paresseux quand vous réfléchissez: vous n'en avez pas envie, c'est trop de travail. Cette attitude vous empêche de faire appel à votre curiosité. Pensez seulement au nombre de fois où les jeunes enfants demandent "pourquoi » et avec quelle intensité ils sont curieux. Retrouvez un peu de cette curiosité ! Pour ce faire, soyez prêt à réfléchir.

Donnez-vous la peine d'être curieux. Gardez-vous du temps pour réfléchir. Pensez à toutes sortes de choses qui semblent n'avoir aucun lien avec votre travail, vos devoirs ou votre expérience immédiate. Laissez votre esprit vagabonder et explorer à nouveau.

\section{La peur}

La peur est l'assassine par excellence de l'esprit créatif. L'angoisse de faire une erreur ou d'avoir l'air fou étouffe toute pensée imaginative. Les jeunes enfants n'éprouvent pas une telle angoisse et vont d'un pas assuré là où les adultes n'osent pas aller. Les gens créatifs attribuent leur créativité au fait qu'ils n'ont pas peur d'avoir l'air stupide.

Pour être un penseur créatif, vous devez prendre des risques. Vous devez être courageux. Les gens créatifs explorent une tonne d'avenues, dont la plupart ne les satisfont pas. Le fait qu'ils abordent les problèmes de façon unique rend plusieurs de leurs analyses et de leurs propositions étranges aux yeux des autres, mais cela signifie également que leurs solutions aux problèmes seront originales et uniques, et auront du succès. 


\section{La cohérence/la constance}

Oscar Wilde (1854-1900) a déjà affirmé que « [la] logique est le dernier refuge des gens sans imagination. » Il entendait par là que les gens qui pensent de manière excessivement linéaire ou rigide sont incapables de créativité. Pour résoudre des problèmes de façon créative, vous devez avoir recours à différentes façons de penser. Vous devez penser latéralement (en faisant des associations et en cherchant des similitudes et des liens), ainsi que verticalement ou logiquement. Vous devez vous imaginer le problème à l'aide de symboles et d'images, et pas seulement au moyen d'un raisonnement verbal.

Pour arriver à des solutions, vous devez sortir des sentiers battus. C'est plus important que de s'entêter à essayer de trouver la bonne réponse.

\section{Les stéréotypes}

Faire usage de stéréotypes, c'est regrouper les choses sur la base de notre perception de ce qu'elles ont en commun. Ce n'est pas nécessairement une mauvaise approche : nous le faisons tous les jours quand nous organisons notre vie. Cependant, cette pratique peut nuire à la créativité si elle embrouille les subtilités ou fait abstraction des différences. La pire façon d'utiliser les stéréotypes est de penser en noir et blanc, c'est-à-dire de diviser les gens et les données en catégories hautement simplistes et restrictives, comme le vrai et le faux, le bien et le mal, nous et eux, le connu et l'inconnu.

Les études postsecondaires tentent d'aller au-delà des stéréotypes en exigeant des étudiants qu'ils cherchent les subtilités, les ambiguïtés et les différences. Ils sont ensuite plus à même de réorganiser l'information pour la classer dans de toutes nouvelles catégories, plus réfléchies, plus complexes et plus significatives.

Cependant, les études causent parfois l'inverse. Les méthodes d'enseignement dogmatiques, le manque d'ouverture de certaines disciplines et les théories trop rigides peuvent empêcher les nouvelles idées de se présenter à l'esprit.

\section{La compression/la réduction}

Nous avons généralement tendance à réduire un problème jusqu'à ce que nous le trouvions assez petit pour nous y attaquer. En fait, c'est 
une réaction tellement automatique que nous n'avons même pas le temps de percevoir le problème autrement ou d'envisager d'autres options. Nous avons trop hâte de nous en débarrasser. Faire preuve de créativité implique d'abandonner complètement cette habitude. Vous devez étendre le territoire du problème, l'envisager de différentes façons et sous différents angles. Vous voudrez évidemment finir par le résoudre, mais vos solutions seront plus intéressantes si vous le grossissez d'abord. Vous ne pourrez pas être créatif si vous le circonscrivez trop rapidement; vous ne pourrez pas faire la lumière dessus si vous ne le regardez pas sous toutes ses coutures; vous ne pourrez pas avoir d'idées créatives si vous ne jouez pas avec plusieurs approches.

Une des meilleures façons de grossir un problème est de l'examiner à la lumière conceptuelle d'une théorie (voir les chapitres

\section{Un mot sur la formation en commerce}

Nous croyons que la formation en commerce souffre énormément de sa tendance à comprimer et réduire les problèmes. Elle compte trop sur la méthode de l'étude de cas et sur la pensée pragmatique. Les études de cas sont des rapports en profondeur au sujet d'une situation particulière ou d'un événement, et permettent de mieux comprendre ce qui est arrivé et pourquoi. Elles ont toutefois tendance à fossiliser l'information et les exemples à coup de formules simplistes. Dès le départ, la solution préférée est incluse dans le problème. Conséquemment, une fois que les étudiants en commerce ont compris cette approche, ils l'appliquent mécaniquement et systématiquement, sans se demander s'il s'agit de la meilleure façon de faire. Avoir des préoccupations strictement pragmatiques (faire des profits) constitue un manque de vision, ce qui met en évidence plusieurs dilemmes auxquels font face les individus, les sociétés commerciales et les gouvernements. Trop mettre l'accent sur les profits mine les possibilités créatives qui pourraient contribuer à la viabilité à long terme des organisations, sans parler de l'effet qu'a cette approche sur les environnements de travail. La résolution de problèmes conventionnelle doit être utilisée en temps et lieu. Si les écoles de commerce veulent réellement que leurs étudiants fassent preuve de créativité en matière de résolution de problèmes, comme elles le prétendent souvent, elles doivent inclure la pensée créative de manière plus formelle dans leurs programmes. 
précédents). Beaucoup de théories sont révolutionnaires, pas nécessairement parce qu'elles encouragent un changement radical, mais parce qu'elles transforment notre façon de voir le monde. Être créatif exige que vous pensiez à des niveaux très profonds, ceux qui caractérisent la compréhension théorique. Par contre, assurez-vous de ne pas perdre votre angle ludique, si nécessaire à l'application créative de théories.

\section{Techniques créatives de résolution de problèmes}

Maintenant que vous avez une meilleure idée de quelques-uns des obstacles qui vous guettent, nous pouvons aborder les techniques à utiliser pour améliorer votre créativité. Souvenez-vous que tout le monde est capable de pensée créative. L'originalité est beaucoup plus répandue que vous ne le croyez. Le truc, c'est d'arriver à l'exploiter. Voici comment faire.

1. La tâche première, la plus difficile, mais également la plus amusante, est de recouvrer votre curiosité et votre capacité à vous émerveiller par rapport au monde et aux gens. Il n'est pas évident de le faire au sein du train-train quotidien, où l'usage et les habitudes vous ont rendu rigide. C'est pourquoi vous devez reprogrammer chez vous ce processus, en lisant, en réfléchissant et en explorant des thèmes qui vous sont moins familiers. Lire, regarder des émissions de télévision, aller au cinéma ou discuter avec des gens de sujets qui vous intéressent, mais qui ne sont pas nécessairement utiles, sont les meilleures façons de le faire.

Quand vous allez au-delà des limites connues de votre esprit pour explorer des territoires moins connus, vous vous donnez matière à jouer avec l'information. L'effet qu'a systématiquement la nouvelle information sur les autres aspects de votre vie, en vous permettant de faire des connexions originales et d'envisager de nouvelles possibilités, est étonnant, voire magique. Ne négligez donc jamais les cadres théoriques que vous apprenez à connaître au cours de vos études postsecondaires ; ils demeurent des façons potentiellement nouvelles de voir et d'explorer le monde.

Parfois, vous n'avez même pas besoin de sources externes de stimulation pour vous revitaliser. Si vous vous réservez du temps pour réfléchir, pour méditer ou pour simplement être, vous parviendrez peut-être à faire momentanément le vide pour permettre à de nouvelles idées de se manifester. Si votre esprit est continuellement 
occupé par votre façon habituelle de penser, il est difficile d'y faire entrer quoi que ce soit de nouveau et d'emballant.

Il va sans dire que ce processus exige d'avoir du temps libre. Avoir un mode de vie équilibré est essentiel à la créativité. Comme le dit le proverbe, à trop travailler, on s'abrutit.

2. La seconde technique que nous suggérons vise à secouer vos approches et vos habitudes mentales. Celles-ci sont tellement incrustées dans votre esprit que vous devez vraiment faire un exercice de transformation mentale. Vous pouvez y parvenir en vous habituant à rendre l'étrange familier, une approche qui peut vous permettre d'envisager le monde selon de toutes nouvelles façons. Vous n'avez qu'à utiliser votre imagination ou, si vous pouvez vous le permettre, partir en voyage à l'étranger.

Un anthropologue, un jour, a décrit le rite étrange d'une tribu: les hommes visitaient quotidiennement une petite salle bien éclairée. Ils s'y raclaient une couche de peau du visage à l'aide d'une lame aiguisée. Ils se coupaient parfois par accident et devaient éponger le sang avec un linge. Malgré les dégâts et la douleur, ils se soumettaient à cette procédure barbare tous les jours.

Qu'a donc décrit l'anthropologue? Avez-vous pensé d'emblée qu'il s'agissait de l'acte quotidien de se raser dans une salle de bain? Remarquez la manière dont ce comportement banal devient un rituel étrange quand on le dépouille de son caractère familier. Maintenant, pensez aux multiples façons d'enrichir la compréhension que vous avez de votre quotidien en le rendant moins familier. La plupart des étudiants sont aux études pendant au moins quatre ans avant de pouvoir travailler dans un environnement professionnel. C'est bizarre, n'est-ce pas? Beaucoup de gens travaillent dans un bureau, ne vivent que pour le weekend et détestent leur emploi. N'est-ce pas là une façon étrange de vivre? Un paysan médiéval ou un guerrier d'une tribu le penseraient assurément.

Et qu'en est-il du magasinage? Nos ancêtres trouveraient peutêtre cette activité curieuse, obsessionnelle et psychologiquement troublante. En fait, être un consommateur n'est probablement pas un mode de vie très sensé! Croyez-vous qu'une personne des siècles passés comprendrait l'expression «magasiner jusqu'à épuisement » ?

3. Attendez avant de porter un jugement. Nous avons tendance à définir beaucoup trop rapidement un problème et sa solution. Il s'agit là d'une bonne façon d'adopter un comportement non critique et non créatif. Pour apprécier le grand art ou la grande littérature, 
vous devez leur donner du temps et vous retenir de juger. Vous devez explorer le sujet ou le problème de fond en comble. Vous devez les laisser vous parler.

Beaucoup d'étudiants en première année ont du mal avec cette approche. Ils décident rapidement s'ils aiment ou n'aiment pas quelque chose, et ont tendance à rejeter la littérature compliquée ou les idées créatives sous prétexte qu'elles sont ennuyeuses ou incompréhensibles (ou les deux). Ces étudiants doivent apprendre à aborder les nouveaux sujets, les nouveaux textes et les nouvelles disciplines comme un défi à relever, non pas comme un choix de consommateur.

Vous n'apprendrez jamais à penser de manière créative si vous ne donnez pas le bénéfice du doute aux problèmes et aux sujets. Vous ne pourrez pas résoudre les problèmes de manière créative si vous ne plongez pas sous la surface pour les explorer en profondeur.

La pensée créative a beaucoup de points communs avec la pensée critique. Toutes deux se situent à l'opposé de la pensée superficielle. Elles vont autant en profondeur qu'en largeur et sont rarement passives. Elles n'acceptent pas telles quelles les informations ou les idées, mais en cherchent plutôt le sens le moins évident ou caché. Les pensées critique et créative abordent toutes deux l'apprentissage de manière hautement réflexive. Elles se différencient l'une de l'autre en ce que la pensée critique est généralement guidée par des règles logiques, alors que la pensée créative se passe de celles-ci et conserve un élément ludique.

4. Une technique similaire à la précédente vise à interpréter les données ou les problèmes selon des points de vue bizarres ou inhabituels. C'est exactement ce qu'a fait Einstein quand il s'est interrogé sur l'univers. À l'époque, la plupart des scientifiques croyaient que l'univers fonctionnait comme une horloge géante, c'est-à-dire qu'il se comportait comme une machine parfaite et que toutes ses parties étaient connectées de manière linéaire par des liens de cause à effet. Cet univers d'une précision mécanique se fondait sur l'idée incontestée que le temps et l'espace étaient constants.

Einstein a laissé son esprit rendre cet univers mécanique plus flou et problématique. Il a ainsi pu concevoir l'espace comme courbé au lieu de linéaire. De plus, il a imaginé le temps et l'espace non pas comme constants, mais plutôt relatifs à la perception de celui qui les perçoit. Comme il l'a dit, une minute peut paraître aussi longue qu'une heure si vous êtes assis sur un poêle chaud, mais une heure 
peut sembler aussi courte qu'une minute si vous êtes dans les bras de la personne que vous aimez.

Un exemple plus concret de cette technique concerne un défilé qui était mis en scène chaque année par de nombreux groupes communautaires. Préoccupée par la possibilité d'être responsable de dommages ou d'accidents qui auraient lieu pendant le défilé, l'administration municipale a obligé les organisateurs à souscrire une assurance. Puisque les groupes communautaires n'avaient pas les moyens de payer celle-ci, le défilé, pourtant populaire, semblait voué à disparaître.

Au lieu de se résigner, les organisateurs ont trouvé une solution ingénieuse: faire un défilé immobile dans un grand champ. Tant que le défilé ne bougeait pas, aucune assurance n'était nécessaire. Les groupes communautaires ont redéfini le concept de défilé de façon créative pour résoudre leur problème. Le défilé immobile annuel est devenu une réussite sociale et économique.

5. Une autre technique créative éprouvée est l'utilisation de symboles. Les artistes, les chanteurs, les acteurs et les écrivains sont appréciés dans la société, certains deviennent même riches et célèbres, parce qu'ils sont des experts en créativité. Au lieu de présenter l'information logiquement, ils utilisent des symboles, des métaphores et des analogies. Jouer avec les symboles leur permet d'envisager le monde de manière plus profonde, plus riche et plus intéressante.

6. Quand vous êtes aux prises avec un problème ou que vous n'êtes pas capable de produire une seule idée créative (et ce, même si votre vie en dépendait), essayez d'utiliser des prépositions pour redéfinir le problème. Les prépositions sont des mots comme: proche, avec, sous, comme, à, parmi, par-dessus, à travers, au sujet, contre, entre, pour, sur, après, par, sans, vers. Vous en connaissez probablement beaucoup plus.

Disons que votre problème a à voir avec deux éléments majeurs : clients et service. Si vous remplacez le mot « et » par d'autres prépositions, vous créez de toutes nouvelles combinaisons ou équations :

- Clients près service pourrait suggérer que vous devriez rapprocher votre service à la clientèle de vos clients ;

- Clients avec service implique que vos clients vous serviront: ils fournissent de l'information importante sur vos produits;

- Clients sous service évoque l'idée que le service pourrait être un parapluie pour protéger les clients ; c'est une identité de marque intéressante qui offre plusieurs possibilités ; 
- Clients comme service pourrait signifier que les clients offrent un service aux autres clients. Un client satisfait pourrait être un très bon représentant indirect de votre compagnie.

À l'heure qu'il est, vous pensez (déjà) de manière plus créative. Vous avez même peut-être remarqué qu'on peut renverser l'équation en commençant par le service :

- Service avant clients pourrait signifier qu'on doit aller audevant des besoins en services des clients, avant même que ces derniers se présentent pour les réclamer;

- Service par-dessus clients suggère de mettre plus d'accent sur le service et moins sur les ventes. Cela pourrait diminuer les besoins en vendeurs puisque le service fera que les clients reviendront ;

- Service pendant clients implique de fournir un service au moment opportun ;

- Service après clients suppose de s'assurer que le service continue après la vente et que les clients en sont informés.

\section{Configuration créative du problème}

Puisque le problème comme tel fait l'objet de tant de motivation et d'énergie créatives, une approche pour le résoudre est d'en modifier la définition. Comme nous l'avons vu plus haut, vous pouvez y arriver en jouant avec des prépositions, mais il y a d'autres moyens d'utiliser le problème lui-même pour arriver à vos fins.

Le premier moyen, et le plus évident, est de se demander s'il est possible de formuler le problème différemment. Cette étape permet à des solutions originales de surgir. Par exemple, les téléphones cellulaires ont d'abord été inventés pour communiquer en cas d'urgence. Ils étaient gros, difficiles à manipuler et coûtaient cher à produire. Quand on les a redéfinis comme appareils pour texter, leur potentiel a augmenté de manière exponentielle et ils vous permettent maintenant d'accéder à Internet, de trouver vos amis, de vous orienter dans la ville, de surveiller votre état de santé, de prendre des photos, de jouer à des jeux, d'écouter de la musique et plus encore. De la même façon, bien qu'il ne fût pas la première tablette électronique, le $i P a d$ d'Apple a redéfini ce qu'une tablette pouvait et devrait faire, révélant ainsi le potentiel énorme du produit.

Un autre moyen est de problématiser la définition. Quand les groupes communautaires ont redéfini leur défilé, ils problématisaient 
la définition du problème en s'interrogeant sur la nécessité que la responsabilité civile ou les assurances fassent partie du problème.

En se concentrant sur des situations où la responsabilité civile n'avait pas de raison d'être, ils ont évité le piège que posait la formulation originale du problème.

Étendre les limites du problème est aussi une approche efficace. On définit souvent les problèmes de manière trop restrictive, ce qui nuit à l'émergence de solutions créatives. Vous pouvez étendre les limites d'un problème en posant les questions suivantes:

- Y a-t-il d'autres problèmes similaires à celui-ci ?

- Est-il particulier à une situation ou est-il plus général ?

- Que peut-on dire d'autre à ce sujet?

- Quels sont ses significations et ses résultats?

Enfin, un moyen plus facile, et qui ressemble à la problématisation du problème, est l'inversion de celui-ci. On appelle parfois cette approche la pensée janusienne (adjectif dérivé de Janus, dieu romain qui avait deux visages: un qui regardait vers le passé et l'autre, vers l'avenir ; c'est aussi de Janus que vient «janvier»). Selon cette approche, un problème pourrait ne pas en être un du tout si on l'aborde de la bonne façon. Cela signifie également qu'il faut faire ressortir les opposés inclus dans le problème ou dans sa définition.

\section{Maximiser les solutions créatives}

La clé pour faire preuve de créativité est de disposer d'une banque de points de vue variés desquels vous pouvez tirer des solutions. Cela implique de solliciter la contribution du plus grand nombre de personnes possible. Cela signifie également que la collaboration est un moyen formidable pour produire des solutions créatives.

Si plus d'une perspective est concentrée sur un problème particulier, les chances de trouver une solution créative sont multipliées. C'est pourquoi les organisations, qui font souvent face à des changements rapides et à la concurrence internationale, encouragent la formation de groupes collaboratifs ou d'équipes de projet. Ces groupes peuvent être assemblés, défaits et reconstruits de manière à garantir la production des meilleures idées possibles.

Les individus peuvent également créer leurs propres groupes, comme les groupes d'études, dont les membres s'entraident pour préparer et faire leurs devoirs. Si vous faites partie d'un groupe, voici quatre suggestions : 
- Pour éviter que le groupe finisse par adopter une structure hiérarchique, donnez la chance à chacun d'y occuper des rôles différents ;

- Encouragez les membres à remettre en question les solutions ou à se faire l'avocat du diable ;

- Les gens ont tendance à devenir paresseux si on ne les pousse pas. Nommez donc quelqu'un qui sera responsable de secouer les autres pour arriver à des solutions. Comme ce n'est pas une position enviable, puisque personne n'aime être celui ou celle qui harcèle, assurez-vous d'affecter souvent quelqu'un de nouveau à cette tâche!

- Protégez-vous de la pensée de groupe, c'est-à-dire de celle où tout le monde pense de la même façon, pour éviter de devenir prévisible, borné et trop à l'aise.

\section{L'environnement idéal pour penser de manière créative}

Même si le travail d'équipe constitue un moteur de créativité potentiellement utile pour les organisations modernes, il doit avoir lieu dans un environnement sécuritaire et confortable. Dans le monde des affaires, les équipes sont des structures temporaires.

Les gens pourraient trouver que leurs vertus collaboratives sont exploitées aux seules fins de la productivité et du profit. Dans ce genre de situation, le caractère et la créativité en souffrent inévitablement. Les gens peuvent bien faire semblant d'être de bons coéquipiers, machinalement, mais leur créativité ne fonctionne pas à plein rendement.

C'est pourquoi la création d'un environnement continuellement sécuritaire est si importante pour la créativité. Certains environnements sont mieux adaptés que d'autres à cet égard. Les meilleurs d'entre eux encouragent les individus à relaxer et à prendre leurs distances par rapport aux problèmes qu'ils doivent résoudre. Les problèmes les plus difficiles trouvent souvent une solution quand les esprits ont non seulement la permission de s'aventurer dans des territoires qui n'ont pas nécessairement de liens immédiats avec les problèmes, mais qu'ils sont encouragés à le faire. Cette façon de faire libère des habitudes mentales et permet aux idées originales d'apparaître.

Un des auteurs de ce livre a eu le bonheur, il y a plusieurs années, de visiter une compagnie de logiciels de haute technologie, bien connue pour ses produits créatifs. Les employés étaient étonnamment 
détendus. Au lieu d'être enterrés sous une tonne de travail ou de trimer comme des forcenés, ils quittaient régulièrement leur cubicule pour aller discuter les uns avec les autres. Le frigo de la cuisine était rempli de toutes les boissons imaginables (boissons gazeuses, jus et eau minérale), toutes offertes gratuitement. Le bureau principal comprenait une table de billard où les employés jouaient et bavardaient pendant leurs heures de travail.

La création de ce bureau est le résultat d'un exercice de réflexion approfondie. Beaucoup de ses caractéristiques ont été pensées dans le but explicite de faire tomber les barrières qui nuisent à la communication et de permettre aux employés de se défaire de leurs habitudes mentales trop rigides. L'espace physique était propice à la pensée créative et très différent des bureaux fermés et des cubicules isolés.

Évidemment, ce ne sont pas tous les lieux de travail qui ressemblent à cela. Dans la mesure du possible, créez des environnements physiques et mentaux qui vous permettent d'être créatifs. Il est important de faire la différence entre un environnement décontracté et un autre qui est trop confortable et offre trop de distractions. C'est d'ailleurs la raison pour laquelle il est préférable de séparer les espaces de vie des espaces de travail ou, à tout le moins, de limiter le travail à un seul endroit à la maison.

Il est tout aussi important de vous donner du temps pour réfléchir et refaire le plein quand vous n'êtes pas au travail. Le divertissement passif peut gruger le temps dont vous avez besoin pour la réflexion plus active et engagée ; éteignez donc le téléphone intelligent, la tablette, la télé et l'ordinateur.

Ne vous isolez pas des gens quand vous travaillez, à moins que ce soit pour accomplir une tâche particulière. Il est sain d'être avec d'autres. Pendant une pause, discuter du travail ou de ce que vous lisez en ce moment stimule la créativité. Et plus vous devenez créatif, intéressant et instructif, plus vous serez attrayant aux yeux des autres personnes créatives et devriez accueillir volontiers toute interaction avec elles.

Il y a toutefois une exception importante à cette règle. Certaines personnes voudront malheureusement profiter de vos talents créatifs sans jamais rien donner en retour. Vous en protéger, ou vous protéger de quiconque a un effet toxique ou négatif, n'est pas méchant ni égoïste. S'associer à des individus négatifs annule toute créativité. Très peu de gens parviennent à conserver leur propre créativité dans un environnement toxique. 
Bien sûr, certains environnements positifs peuvent également étouffer la créativité. C'est une bonne idée de les reconnaître et de les garder pour les occasions appropriées. Par exemple, vous aimez peut-être sortir en ville avec vos amis. Ne vous en privez surtout pas et amusez-vous! Sachez toutefois que ces sorties n'ont probablement pas grand-chose à voir avec la pensée créative.

Ayez conscience que votre croissance personnelle peut générer des tensions avec vos amis et votre famille. C'est pourquoi ceux qui sont très créatifs se lient souvent d'amitié avec des gens qui le sont tout autant. Ce n'est pas compliqué : une des meilleures façons de devenir créatif et de le demeurer est de passer du temps avec d'autres personnes qui le sont. Souvenez-vous seulement de donner autant de créativité que vous en recevez!

Avec les devoirs, le travail, les obligations familiales et tout le reste, vous pouvez perdre votre capacité à être plus profondément à l'écoute de vous-même et des autres. Il est impossible de faire long feu dans une telle situation et de donner le meilleur de vous-même. Évitez de vous brûler!

$\mathrm{Au}$ fur et à mesure que vous avancez dans vos carrières scolaire et professionnelle, vous devez prendre soin de vous-même et il n'y a pas de meilleur moyen de le faire que de développer votre créativité. Contrairement à beaucoup d'autres aspects fastidieux de la vie, la créativité y ajoute du plaisir et du mystère. De plus, elle ne se limite pas à une activité précise. Vous pouvez l'utiliser dans tout ce que vous faites, de la cuisine à la couture, en passant par la peinture, l'exercice et l'écriture.

Certains restreignent artificiellement leurs élans créatifs à leurs passe-temps ou à leurs loisirs, et étudient et travaillent machinalement. Ils se privent ainsi de la chance extraordinaire de développer et de mettre en pratique des aptitudes qui sont grandement recherchées. Si vous faites preuve de créativité à l'école et au travail, vous serez un employé qui s'ennuiera beaucoup moins et qui sera hautement prisé.

Soyez à l'affût d'occasions de nourrir votre imagination. Protégez l'enfant créatif en vous. Donnez-vous le temps de rêvasser.

\section{Notes}

1. Donald A. Norman, Things That Make Us Smart: Defending Human Attributes in the Age of the Machine, New York Addison-Wesley, 1993, p. 17. Traduction libre.

2. Ulrich Kraft, «Unleashing Creativity », Scientific American Mind, vol. 16, n 1, avril 2005, p. 16-23. 
Page blanche conservée intentionnellement 


\title{
Trouver et décrocher l'emploi idéal
}

\author{
«Persévérez et il est fort possible que vous \\ tombiez sur quelque chose, peut-être au \\ moment où vous vous $\mathrm{y}$ attendez le moins. \\ Je n'ai jamais entendu parler de quelqu'un \\ qui soit tombé sur quelque chose alors qu'il \\ était assis. » - Charles Kettering
}

$\mathrm{E}^{\mathrm{n}}$ n tant qu'étudiant ou étudiante qui obtiendra sous peu son diplôme, ou qui vient tout juste de l'obtenir, il est fort possible que vous n'ayez aucune idée où chercher les bons emplois. L'étude de certains aspects des développements actuels dans le monde des affaires et de la politique peut cependant vous emmener dans la bonne direction. Si une certaine industrie est en plein essor ou qu'un certain sujet fait tout à coup les manchettes, beaucoup d'emplois peuvent soudainement apparaître, de conseiller en ressources humaines à programmeur-analyste, en passant par plusieursautres. Certains de vos professeurs peuvent également vous guider vers des possibilités d'emploi. Après tout, se tenir au courant de ce qui se passe dans leur domaine d'expertise fait partie de leur travail.

Vous devez toutefois être conscient que le marché de l'emploi change très rapidement. Ne choisissez pas une majeure, un programme ou une profession seulement parce que vous croyez que vous aurez l'embarras du choix quand viendra le moment de trouver un emploi. 
Une bien meilleure approche consiste à faire ce qui vous intéresse et à garder l'œil ouvert sur tout lien que vous pouvez faire entre vos intérêts et les occasions d'emploi qui se présentent. Comme nous le suggérons dans les chapitres précédents, servez-vous de vos années d'études postsecondaires pour déterminer quel genre d'emploi vous procurera le plus de satisfaction et correspondra à vos habiletés et intérêts. Sachez ce que vous aimez et ce dans quoi vous êtes bon, pas nécessairement ce que vous voulez devenir.

Nous avons insisté sur le fait que quatre ensembles de compétences mènent au succès à l'école comme au travail :

- la communication (lire, écrire, parler, écouter) ;

- la capacité à apprendre et à résoudre des problèmes ;

- le travail d'équipe, y compris l'entregent (éthique, attitude positive, responsabilité) ;

- la capacité à s'adapter aux circonstances changeantes et à transférer ses connaissances à de nouvelles situations.

Laquelle de ces compétences constitue votre force? Pour déterminer où vous vous épanouirez dans le monde professionnel, vous devez vous donner une note pour chacune.

Ceux qui choisissent une carrière pour des raisons économiques et de sécurité d'emploi ont souvent une très mauvaise compréhension de qui ils sont et de ce qu'ils veulent dans la vie. Si leur plan de carrière tourne au vinaigre, ils seront à la dérive jusqu'à ce qu'ils se soumettent à un long examen de conscience.

Il est beaucoup plus facile de trouver du travail que de découvrir ce qui vous correspond professionnellement. Trop de gens sont malheureux parce qu'ils occupent des emplois qui ne correspondent pas à leur personnalité. Trop de gens sont coincés dans ces emplois pour des raisons financières et de sécurité d'emploi. Ne tombez pas dans ce piège. Réfléchissez bien au genre de travail qui correspond à votre tempérament et utilisez votre expérience postsecondaire pour vous guider et vous aider dans ce processus de découverte de vous-même.

Ne vous limitez pas aux titres des emplois ou aux attentes des autres. Si vous vous donnez la peine de faire des connexions, vous verrez que vos intérêts et vos talents répondent aux besoins du marché du travail de plusieurs façons. Les auteurs de ce livre sont peut-être des chercheurs professionnels, mais leurs compétences sont flexibles et le chemin qui les a menés à leur emploi actuel a été tout, sauf direct.

Par exemple, l'un des deux a été, successivement, administrateur, directeur du centre de placement d'une école de commerce 
prestigieuse et président d'une compagnie qui assurait la communication entre les gouvernements et les universités. L'autre auteur a travaillé pendant près de dix ans comme conseiller en politiques auprès du gouvernement, a occupé un poste de consultant à Ottawa et a enseigné en Asie et en Europe. Tous ces emplois étaient merveilleux et, pour rien au monde, ils y changeraient quoi que soit.

Personne n'obtient son emploi idéal immédiatement après avoir terminé ses études. Vous mettrez des dizaines d'années avant d'occuper l'emploi qui vous convient parfaitement. Le trajet pour y arriver sera plus enrichissant si vous avez une destination en tête, basée sur vos intérêts et vos capacités.

\section{L'importance du réseautage}

Les réseaux sont formés des relations humaines informelles, difficilement perceptibles, qui gouvernent l'ensemble de ce qui se passe au travail. Peu importe à quel point une organisation peut sembler bureaucratique, les décisions importantes, comme les embauches et les promotions, proviennent habituellement, en grande partie, du réseautage. Les experts en carrière estiment que plus de $80 \%$ des emplois ne sont jamais affichés. Quand ils ont un poste à pourvoir, les employeurs et les gestionnaires se tournent invariablement vers leurs réseaux.

C'est logique, puisque la préoccupation principale des employeurs est de rapidement trouver la bonne personne. Après tout, ils ont tendance à embaucher de nouveaux employés seulement après qu'une tonne de travail urgent s'est accumulée! Afficher un poste, répondre à des douzaines de courriels, lire des centaines de $C V$, sélectionner et faire passer des entrevues aux candidats, valider les références et négocier un contrat de travail prend beaucoup de temps. Et il n'y a aucune garantie qu'ils feront le bon choix. Ainsi, les employeurs préfèrent embaucher quelqu'un qu'ils connaissent déjà ou qui leur est recommandé par quelqu'un en qui ils ont confiance ${ }^{1}$.

L'objectif premier du réseautage est de vous faire connaître, vous et votre $\mathrm{CV}$, par le plus grand nombre de personnes possible. S'ils entendent parler d'une possibilité d'emploi, ils penseront à vous. Cela ne signifie pas que le poste sera à vous automatiquement, mais seulement que vous pourriez être considéré pour l'emploi.

Le réseautage efficace fournit donc beaucoup de points de contact potentiels au cours de votre carrière; votre réseau pourrait se 
révéler votre principal atout en matière de recherche d'emploi ou de promotion.

Les statistiques viennent appuyer cette affirmation. Comme vous le verrez dans le tableau ci-dessous, un sondage fait auprès de milliers d'individus au Canada et aux États-Unis a révélé que près de la moitié d'entre eux ( $46 \%$ ) ont trouvé un emploi par réseautage, c'està-dire par personnes interposées ${ }^{2}$.

\begin{tabular}{|l|c|}
\hline Ce qui a mené à l'emploi & Pourcentage \\
\hline Agence/recruteur & 14 \\
\hline Approche directe & 7 \\
\hline Babillards électroniques/sites Web & 25 \\
\hline Journaux/périodiques & 1 \\
\hline Autre & 7 \\
\hline Réseautage & 46 \\
\hline
\end{tabular}

Former un groupe dont les membres coopèrent pour trouver des emplois et des employeurs est une façon particulièrement efficace et novatrice de réseauter. Il permet de rassembler de l'information et des pistes à explorer. Vous-même n'êtes peut-être pas la bonne personne pour l'emploi, mais quelqu'un dans votre groupe pourrait l'être, et vice versa. C'est une stratégie qui fonctionne bien pour les étudiants qui viennent tout juste de terminer leurs études ou qui sont sur le point de le faire. Établissez toutefois bien votre groupe avant de perdre contact avec plusieurs de vos camarades d'études postsecondaires.

Il est en effet préférable de commencer à réseauter le plus tôt possible. La plupart des écoles de commerce encouragent leurs étudiants à réseauter dès qu'ils entrent en première année, sous prétexte que leurs camarades de classe finiront, un jour, par être leurs contacts. Eh bien, ce qui fonctionne pour les étudiants en commerce fonctionne aussi pour vous. Les médias sociaux sont particulièrement utiles à cet égard, rendant le réseautage plus facile que jamais (nous reviendrons sur les médias sociaux plus tard dans ce chapitre et dans le suivant).

Il n'est jamais trop tard pour commencer à réseauter. Si vous n'avez pas déjà une liste de contacts, créez-en une dès maintenant. Développer un réseau qui peut vous aider à trouver un emploi professionnel prend au moins quatre mois. Les réseaux sont fragiles, toutefois, et s'écrouleront si vous ne les entretenez pas correctement. Les construire lentement ajoutera à leur solidité et à leur stabilité. 
Les réseaux professionnels exigent de votre temps et de votre talent. Vous avez besoin de temps pour faire des entrevues d'information (dont nous traitons ci-dessous) et on pourrait vous demander de prêter main-forte lors des activités que vos contacts organisent. Toute contribution professionnelle de ce type améliorera l'ensemble de vos compétences ainsi que la taille et la signification de votre réseau. Soyez fiable et faites un effort réel. Le bouche-à-oreille fera ensuite le travail de communiquer qui est digne de confiance et qui ne l'est pas.

\section{Les curriculum vitæ}

Votre CV est le reflet de votre professionnalisme. Un CV impressionnant suggère une personnalité impressionnante. Un CV dont le vocabulaire est actif et projette l'image d'un individu qui a de l'initiative reflète le très recherché employé motivé. Un $\mathrm{CV}$ bien organisé témoigne d'une personne logique et efficace.

On a probablement écrit plus d'absurdités sur les CV que sur n'importe quel autre sujet. Il n'y a pas un modèle de CV qui est plus efficace qu'un autre, même si plusieurs personnes voudraient le croire. Un CV n'a pas à être limité à une seule page. Un CV de deux pages, organisé de manière élégante, se lira beaucoup plus facilement qu'une page sur laquelle vous avez tenté de tout entasser. L'utilisation d'une police de couleur est même permise, tant que vous n'exagérez pas.

La caractéristique la plus importante d'un bon CV est d'être le reflet de la personne dont le nom se trouve au haut de la page. Le CV est une façon de vous faire connaître. À quoi bon y décrire quelqu'un de différent de qui vous êtes vraiment?

Mentir ou exagérer dans un CV pourrait vous permettre d'obtenir un emploi, mais vous risquez que votre patron et vos collègues découvrent plus tôt que tard que vous n'êtes pas la personne que vous prétendiez être. Il est également possible que le travail en question ne corresponde pas à vos talents ou à vos intérêts. Demeurer vousmême, et vous représenter fidèlement, reste la meilleure façon de vous assurer de trouver la satisfaction au travail, dans votre carrière et dans votre vie.

La composition d'un CV solide et d'une bonne lettre d'accompagnement requiert toutefois plus que de l'exactitude et de la sincérité. Elle demande quelques-unes des habiletés utilisées lors de la rédaction d'une bonne dissertation. Vous devez vous mettre à la place du lecteur et faire preuve de clarté et de concision. 
La plupart des conseils sur la rédaction d'un CV relèvent du gros bon sens, en particulier si vous considérez la façon dont un employeur le lira :

- Si vous êtes un étudiant, ou un récent diplômé, soulignez votre formation en incluant beaucoup de détails, puisqu'au fond, c'est votre formation qui représente le mieux qui vous êtes en ce moment ;

- Écrivez le nom de votre programme d'études exactement comme il apparaît sur votre relevé de notes ;

- Si vous travaillez à temps plein depuis un moment, mettez l'accent sur votre expérience de travail, mais souvenez-vous que votre formation aussi est essentielle ;

- Incorporez tout bénévolat en lien avec l'emploi. Cette expérience est tout aussi importante que toute autre expérience de travail et témoigne du genre de personne que vous êtes ;

- Utilisez un vocabulaire actif qui met l'accent sur vos réalisations, comme : «développer», "gagner», « rechercher», « étudier », " évaluer », " améliorer », " apprendre » et « analyser», au lieu d'utiliser un vocabulaire passif comme: «tâches » et « responsabilités ";

- Faites la liste de vos passe-temps ou de vos autres intérêts seulement s'ils sont en lien avec le poste;

- N'incluez pas de références. Si un employeur est intéressé, il vous les demandera ;

- Ne faites jamais d'erreurs d'orthographe ou de grammaire. Les CV qui en contiennent finissent toujours au recyclage ;

- Joignez toujours une lettre d'accompagnement qui explique pourquoi vous êtes la personne tout désignée pour le poste, même si on ne vous en demande pas.

La plupart des employeurs n'ont aucune tolérance pour les CV peu soignés ou dont la mise en forme laisse à désirer. Si vous ne savez pas comment créer le vôtre et n'avez pas le temps de l'apprendre, embauchez quelqu'un qui le fera pour vous. Si le coût vous semble rédhibitoire, rappelez-vous simplement qu'un travail imparfait vous fait perdre votre temps et votre argent. Encore mieux, cherchez des échantillons en ligne, demandez à vos amis et à votre famille de vous en fournir, et créez votre propre CV. Vous trouverez de très bons exemples dans ce chapitre pour vous aider.

Vous pouvez améliorer de façon exponentielle les chances que votre $\mathrm{CV}$ provoque une réaction positive en y incorporant la preuve 
que vous possédez les caractéristiques recherchées, comme de bonnes habiletés en communication, la capacité à travailler en équipe, à résoudre des problèmes et à réfléchir de manière critique et créative, à travailler de façon autonome et à mener plusieurs tâches de front. Vous trouverez ci-dessous des exemples créatifs à cet effet.

\section{Exemples de curriculums vitæ}

Les trois CV qui se trouvent à l'annexe 1, à la fin de ce chapitre, sont des modèles que vous pourriez utiliser. Bien que les noms aient été changés, il s'agit de vrais CV qui ont permis à des étudiants de décrocher des emplois à temps partiel, d'été et à temps plein.

Ces CV ont eu du succès parce qu'ils sont le produit de beaucoup d'efforts, de temps et de réflexion. Les employeurs potentiels y trouvent le genre d'informations dont ils ont besoin pour décider si la personne mérite de passer une entrevue. Vous remarquerez qu'ils sont tous différents, puisque chaque personne est unique et a des objectifs d'emploi qui lui sont propres.

\section{Quelques trucs pour la rédaction d'un curriculum vitæ}

Truc 1: Utilisez la fonctionnalité tableau de votre logiciel de traitement de texte pour mettre en forme votre CV. Une fois que cela est terminé, cachez les bordures. Cela vous permettra d'organiser votre $\mathrm{CV}$ de manière originale et de le modifier facilement par la suite.

Truc 2: Utilisez votre courriel étudiant. N'importe qui peut se créer une adresse Gmail, mais ce n'est pas tout le monde qui est aux études. Vous pouvez paramétrer votre compte courriel étudiant pour qu'il fasse suivre automatiquement tous vos messages à un autre compte, si vous le voulez. Oh, et en passant, les noms ridicules comme megadeath@gmail.com ne donnent pas une bonne idée de votre professionnalisme aux employeurs potentiels.

Truc 3: Mettez toujours l'accent sur le poste que vous avez occupé plutôt que sur votre employeur ou la compagnie pour laquelle vous avez travaillé. Vos futurs employeurs s'intéresseront à ce que vous avez fait plutôt qu'aux endroits où vous l'avez fait. Il en est de même pour votre formation. Les employeurs potentiels s'intéressent moins à l'endroit où vous avez fait vos études qu'au programme particulier que vous avez suivi et aux résultats que vous avez obtenus. 
Le premier CV (page 195) est celui d'un étudiant qui a changé de programmes, d'universités, de pays et d'employeurs, et qui est sur le point d'obtenir son diplôme. Remarquez à quel point ce cheminement est bien présenté. Remarquez également tous les détails sur sa formation, y compris sa moyenne pondérée. Dans votre propre $\mathrm{CV}$, incluez les titres de vos dissertations, si c'est pertinent.

Le deuxième CV (page 198) est celui d'une étudiante de deuxième année. Remarquez les choix qu'elle a faits dans la construction de son $\mathrm{CV}$, comme celui de ne pas faire mention de ses études secondaires. Le dernier CV (page 200) illustre comment une étudiante qui a une expérience de travail limitée et qui vient tout juste de commencer ses études a présenté son historique de travail jusqu'à maintenant.

\section{La lettre d'accompagnement}

Si les CV sont nécessaires à l'obtention d'un poste professionnel, ils ne sont jamais aussi importants que les lettres d'accompagnement. $\mathrm{Si}$ on ne vous demande pas de soumettre une lettre d'accompagnement quand vous postulez un emploi, le travail que vous y feriez n'est probablement pas digne d'un professionnel.

Le CV fait état de vos compétences, de vos réalisations et de votre caractère. La lettre d'accompagnement explique pourquoi vous êtes le bon choix pour ce poste en particulier. Voyez cette lettre comme une espèce de demande en mariage. Les employeurs sont à la recherche de quelqu'un dont la contribution potentielle correspondra bien à leurs besoins.

Les recruteurs d'expérience et les employeurs qui s'y connaissent $s^{\prime}$ intéresseront davantage à votre lettre d'accompagnement qu'à votre $\mathrm{CV}$. Ils voudront y trouver des indices de votre personnalité et les raisons pour lesquelles vous avez choisi de postuler un emploi chez eux. S'ils découvrent que vous connaissez leur réputation et leurs réalisations, ils seront flattés. Ils seront agacés s'ils soupçonnent que vous postulez un peu partout et au hasard. Par-dessus tout, ils s'attendront à ce que vous ayez fait les recherches appropriées à leur sujet et au sujet du poste.

Faites en effet des recherches sur la compagnie ou l'organisation où vous postulez. Avant même de rédiger votre lettre d'accompagnement, vous devriez en savoir autant que possible sur ceux qui la liront. Quelle est la philosophie de l'organisation? A-t-elle un énoncé de mission? À quels problèmes fait-elle face en ce moment? À quels problèmes 
pourrait-elle faire face dans l'avenir (comme la concurrence ou des changements dans les grandes tendances démographiques)? Votre lettre d'accompagnement sera beaucoup plus efficace si elle fait état de votre appréciation des réalisations, de la culture et des défis de l'organisation.

La majorité de l'information de base à ce sujet se trouve sur Internet et dans d'autres sources, comme les rapports annuels. Toutefois, puisque vous êtes un étudiant qui s'y connaît bien en recherche et en pensée critique, vous pouvez ajouter de la valeur à l'information que vous amassez. Cela vous permettra de vous distinguer. Un fait intéressant que vous avez appris en rédigeant une dissertation s'appliquera peut-être à la compagnie ou au poste. Essayez de l'inclure dans votre lettre d'accompagnement.

S'il y a eu affichage du poste, répondez-y en utilisant les mêmes formulations et le même ton. Essayez toujours de montrer que vous avez les caractéristiques demandées. Répondez systématiquement à chacune d'elles. N'utilisez jamais une lettre d'accompagnement générique. Adaptez-la plutôt au poste affiché. Si vous trouvez que ça représente beaucoup de travail, dites-vous que les chances d'être présélectionné avec une lettre générique sont virtuellement nulles. Quelle perte de temps!

Prenez en considération le ton et la langue de l'affichage. Est-ce qu'il s'en dégage du dynamisme ? Sont-ils précis, conservateurs, détaillés, formels, informels? Le ton et la langue sont souvent une indication de ce que l'organisation recherche. Votre lettre d'accompagnement sera plus efficace s'il s'en dégage les mêmes éléments.

Dites aux employeurs potentiels ce que vous pouvez leur apporter. Les lettres d'accompagnement des étudiants s'attardent typiquement à ce qu'une organisation peut fournir et demeurent vagues quant à ce qu'ils peuvent eux-mêmes apporter comme contribution à la compagnie. Les recruteurs d'entreprise éliminent systématiquement ce genre de demande d'emploi. Ils cherchent des candidats motivés et qui font preuve d'initiative en résolution de problèmes, avec des connaissances récentes, acquises au cours de leurs études.

Votre objectif doit être de dire comment vous pouvez faire le travail. La lettre doit être «tournée vers l'avenir». Un employeur potentiel est en effet beaucoup plus intéressé à savoir ce que vous pourrez accomplir quand il vous aura embauché qu'à ce que vous avez fait jusqu'à maintenant.

Rédigez votre lettre avec grand soin. Vous seriez étonné d'apprendre combien de CV sont rejetés parce qu'ils sont accompagnés 
de lettres mal écrites et remplies d'erreurs de grammaire. Plus d'une faute d'orthographe dans une lettre d'accompagnement ou un $\mathrm{CV}$ garantit généralement un aller simple vers la poubelle!

N'essayez pas d'être quelqu'un que vous n'êtes pas. Faites des demandes d'emploi à des compagnies qui cherchent quelqu'un comme vous, avec vos caractéristiques. Même si vous utilisez avec succès les techniques mentionnées plus haut et obtenez un emploi, vous ne serez heureux que s'il correspond à qui vous êtes vraiment.

\section{Format de la lettre d'accompagnement}

La plupart des lettres d'accompagnement partagent le même format de base (comme tous les documents d'ailleurs, qu'il s'agisse de dissertations ou de poèmes). Vous en trouverez un exemple à la page 201. Ne vous sentez toutefois pas obligé de le suivre aveuglément. Adaptez-le plutôt à votre propre situation. L'objectif de la lettre d'accompagnement est de transmettre de l'information à votre sujet, qui n'apparaît pas dans votre $\mathrm{CV}$ et qui aidera l'employeur à prendre une décision.

Essayez de trouver le nom du gestionnaire qui embauche et utilisez-le. Gardez en tête qu'au final, c'est cette personne qui décidera qui sera embauché ou non, et non quelqu'un du département des ressources humaines. Vous devrez peut-être soumettre votre demande au département des ressources humaines, mais savoir qui est responsable de l'embauche (votre futur patron) confère à votre lettre d'accompagnement une qualité particulière et atteste le sérieux de votre démarche.

Commencez par un paragraphe d'introduction dans lequel vous énoncez votre intérêt pour le poste et essayez de capter l'attention de votre lecteur. Vos formulations doivent traduire un intérêt réel, sans toutefois manquer de professionnalisme. Une aguiche efficace pour le lecteur est souvent l'inclusion de vos connaissances sur la compagnie ou d'un compliment (la sincérité est de mise, mais pas la flatterie!). Les premières lignes de la lettre pourraient ressembler à ceci :

J'ai récemment appris qu'un poste contractuel de six mois comme assistant débutant en marketing, avec une spécialisation en médias sociaux, est disponible aux Entreprises TRK. 
Dans le paragraphe suivant, faites l'énoncé de vos forces et expliquez ce que vous pouvez apporter comme valeur ajoutée à l'organisation. Un récent diplômé pourrait écrire :

Au cours de mon baccalauréat en anglais et en histoire à mon collège universitaire, j'ai eu la chance d'étudier l'utilisation et les effets des médias sociaux dans deux cours de quatrième année. J'ai travaillé à temps partiel pendant quatre ans comme vendeur pour une compagnie de la taille des Entreprises TRK, où mes gestionnaires ont qualifié mon travail « d'excellent, avec une attention marquée pour les détails ».

Dans le paragraphe suivant, présentez un résumé détaillé de votre formation et de votre expérience pertinentes. Par exemple, vous pourriez nommer des cours précis que vous avez suivis et tout attribut particulier qui servirait à convaincre un employeur potentiel que vous êtes le candidat idéal :

Au cours de mes études de baccalauréat, mes cours d'anglais portaient sur la façon dont les auteurs utilisent la parole écrite pour communiquer des sentiments et des émotions complexes. Dans mes cours d'histoire de troisième année, j'ai étudié la manière dont les tendances démographiques canadiennes des 50 dernières années ont modelé, et modèlent toujours, les développements sociaux et économiques. J'ai utilisé certaines des connaissances acquises au cours de mes études ainsi que celles que j'avais déjà sur les médias sociaux pour créer mon propre site Web : monsiteweb.com.

Si nécessaire, vous pouvez ajouter un court paragraphe qui aborde tout problème que pourrait percevoir un employeur. Par exemple, vous devez expliquer tout manque ou tout intervalle dans votre cheminement :

Entre ma troisième et ma quatrième année d'études, j'ai voyagé et travaillé pendant 14 mois en Asie de l'Est avec ma sœur. Ce fut pour moi une merveilleuse occasion d'apprendre, qui m'a permis d'être exposé à une grande variété de cultures et d'attitudes. Bien que difficile par moments, en particulier quand j'ai travaillé pendant quatre mois dans un restaurant chic de Beijing, 
cette expérience m'a beaucoup appris sur la façon de surmonter les obstacles et d'interagir avec diverses personnes.

Si possible, ajoutez un paragraphe qui aborde des problèmes que pourrait avoir l'organisation. Il s'agit là d'une bonne façon de mettre en pratique de nouvelles connaissances ou des techniques apprises pendant vos études ou ailleurs :

J'ai récemment lu, dans le livre Le zen du marketing des médias sociaux : comment mieux bâtir votre crédibilité, que les médias sociaux sont très efficaces pour attirer les consommateurs potentiels, mais moins pour augmenter le chiffre d'affaires. Je suis grandement intéressé à travailler avec le personnel expérimenté en marketing des Entreprises TRK pour aider à convertir les consommateurs potentiels en clients à long terme.

Ajoutez une ou deux phrases qui expliquent comment vous entendez faire un suivi avec eux. Vous devez démontrer que vous avez de l'initiative et que vous n'attendrez pas passivement qu'on vous appelle :

C'est avec grand plaisir que je discuterai plus avant du poste et de ma contribution à l'occasion d'une entrevue personnelle. Je vous écrirai ou vous appellerai mardi prochain pour sonder votre intérêt à planifier une telle rencontre.

Concluez en affirmant combien vous êtes reconnaissant qu'on considère votre candidature. Ce remerciement formel peut s'énoncer de plusieurs façons :

Je vous remercie de considérer ma candidature et vous souhaite tout le succès possible pour l'embauche de la personne parfaite pour ce poste important et des plus motivants.

\section{Ce qu'il faut éviter}

Les huit erreurs suivantes nuisent à l'efficacité d'une lettre d'accompagnement :

- Être fade: Vous réussirez difficilement à capter l'attention de votre lecteur si vous utilisez des phrases insipides et 
fades. Rappelez-vous que vous êtes un candidat parmi des douzaines, voire des centaines, d'autres candidats ;

- Parler pour ne rien dire: N'utilisez pas de phrases creuses qui ne veulent rien dire. Ne donnez pas non plus trop $\mathrm{d}$ 'informations qui n'ont rien à voir avec l'emploi. Concentrezvous sur ce dernier et sur la correspondance entre les besoins de l'employeur et vos compétences. Vous pouvez, et devriez, y faire transparaître un peu de votre personnalité, mais il n'est absolument pas nécessaire d'entrer dans les détails ;

- Trop parler de soi : Vous devez parler de vous dans la lettre d'accompagnement, mais l'employeur ou l'organisation doit demeurer au centre du sujet ;

- Faire des fautes de grammaire, de ponctuation ou d'orthographe, ou avoir un style d'écriture pauvre: Comme nous l'avons indiqué plus haut, ces erreurs ressemblent au baiser de la mort. Si vous faites ce genre de fautes, votre demande d'emploi aura été une pure perte de votre temps ;

- Faire preuve de suffisance : Il n'y a qu'un pas à franchir entre expliquer votre valeur potentielle et crier sur tous les toits à quel point vous êtes extraordinaire. Encore une fois, si vous vous concentrez sur les besoins de votre employeur, vous éviterez l'égocentrisme qui mène à la vantardise ;

- Se dénigrer: La fortune sourit aux audacieux. S'il y a un endroit où vous devez faire preuve d'assurance quant à ce que vous pouvez offrir à une organisation, c'est dans votre lettre d'accompagnement et pendant votre entrevue. Tout le monde sait qu'elles servent à vous mettre en valeur et à convaincre que vous êtes le candidat idéal. La timidité n'est pas propre à ceux qui savent bien résoudre les problèmes;

- Pécher par excès d'ambition : L'excès d'ambition est rarement efficace. Ne dites pas à vos employeurs potentiels ce qu'ils devraient faire ou ne pas faire. Ne leur donnez pas une liste des moments où vous êtes disponible pour une entrevue ;

- Traîner en longueur: Une lettre d'accompagnement devrait idéalement faire une page. Il est acceptable de déborder sur une seconde page si votre lettre est bien écrite, mais la plus grande partie de cette deuxième page devrait demeurer blanche.

Vous trouverez un exemple de lettre d'accompagnement à l'annexe 2 , à la fin de ce chapitre. Si vous étiez recruteur, vous inciteraitelle à communiquer avec le candidat pour lui proposer une entrevue? 


\section{Le réseautage et l'entrevue d'information}

Ne laissez pas les livres et les entreprises qui se consacrent aux CV et aux lettres d'accompagnement vous induire en erreur au sujet de l'aspect le plus important de la recherche d'emploi. Le réseautage est plus efficace que de formellement postuler un emploi qui a été affiché, même si vous avez le meilleur CV ou la meilleure lettre d'accompagnement du monde. Mais que faire si vous consacrez chaque heure de votre journée à étudier ou à travailler à temps partiel et n'avez aucune expérience en réseautage? Par où commencer?

Il y a heureusement un mécanisme de réseautage bien établi et compris par tout le monde: l'entrevue d'information. Il s'agit d'une séance de questions et réponses avec quelqu'un qui travaille pour une organisation qui vous intéresse.

Cette personne n'est pas gestionnaire, ni n'a l'autorité de vous embaucher. Il s'agit plutôt de quelqu'un qui aime son travail et qui est prêt à vous accorder quelques minutes de son temps pour vous en parler. C'est une entrevue sans pression aucune pour les deux participants, au cours de laquelle il y a habituellement beaucoup d'enthousiasme partagé. Elle vous permet également de commencer à développer un réseau.

La planification d'une entrevue d'information est relativement simple. La plupart des professionnels savent de quoi il s'agit et se font un plaisir de parler de leur travail. Vos professeurs pourraient probablement vous donner au moins un nom. Vous pouvez également trouver des gens sur LinkedIn, dans un répertoire de courriels en ligne ou en faisant une recherche sur Internet. Cherchez des gens avec qui vous avez des points communs : votre programme d'études, votre école ou toute autre caractéristique.

Dès que vous avez un nom, envoyez un courriel ou téléphonez à la personne pour lui demander dix minutes de son temps, à un moment qui lui convient. Dites-lui que vous voulez seulement en apprendre davantage sur son travail et précisez-lui qu'elle ne s'engage absolument à rien en vous rencontrant. Les professionnels étant déjà assez occupés en raison de leur charge de travail et de la pression qui en découle, rassurez-la également que vous ne prendrez pas plus de dix minutes de son temps.

Habillez-vous de façon appropriée. Puisqu'il s'agit d'une rencontre informelle, vous n'avez probablement pas besoin de porter un complet, un tailleur, une cravate et tout le tralala qui accompagne une 
entrevue formelle. Inspirez-vous de la façon dont ceux qui occupent ce genre d'emploi s'habillent au travail. En cas de doute, choisissez des vêtements plus chics que décontractés.

Certains ont l'impression de ne plus être eux-mêmes en tenue professionnelle. C'est là une attitude très bête et défaitiste. La plupart des professions ont un code vestimentaire, même s'il permet une variété de choix. Les individus qui exercent une profession ne sont pas moins uniques du fait qu'ils se conforment à certaines normes reconnues.

Arrivez tôt. Vous indiquez ainsi que vous respectez le temps de la personne que vous rencontrez. Respectez donc également la durée prévue de dix minutes.

Apportez un bloc-notes et prenez des notes. Vous indiquez ainsi que vous prenez au sérieux ce qu'on vous dira. Posez les questions suivantes :

- Pourquoi avez-vous choisi cette profession?

- Comment avez-vous obtenu votre premier emploi après avoir terminé vos études?

- Qu'est-ce qui vous plaît le plus dans votre travail ?

- Qu'est-ce que vous aimez le moins?

- Si vous deviez donner un seul conseil à quelqu'un qui songe à exercer votre profession, quel serait-il ?

- Serait-il possible de me donner les noms et les coordonnées d'autres personnes qui seraient prêtes à me parler de la profession?

- Est-ce que je peux mentionner votre nom quand je communiquerai avec elles?

Ne vous étonnez pas si la rencontre dure plus longtemps que les dix minutes prévues, à la demande de votre interlocuteur. Après tout, qui n'aime pas parler de soi-même? Veillez toutefois à détecter les signes que la séance est terminée et partez dès que vous les remarquez.

\section{Après l'entrevue d'information}

La première chose à faire après une entrevue d'information est de remercier la personne que vous avez rencontrée de vous avoir accordé un peu de son temps précieux. De retour à la maison, mettez son nom sur une fiche ou créez un dossier électronique à son sujet, et rédigez quelques commentaires sur la rencontre. Profitez du fait que cette dernière est toujours fraîche à votre esprit pour composer et envoyer une 
note de remerciement, que ce soit par courriel ou sur une carte que vous posterez.

Commencez alors à créer votre réseau. En communiquant avec ceux à qui vous aura référé votre interlocuteur, vous aurez bientôt une liste de contacts dans la profession. Communiquez toujours avec tout le monde. Vous vous découvrirez des atomes crochus avec certains d'entre eux et vous sentirez à l'aise de recommuniquer avec eux. Félicitations! Vous avez commencé à développer un réseau.

Entretenez votre réseau, mais faites attention de ne pas tomber sur les nerfs de vos contacts. Si vous faites une entrevue avec quelqu'un qui vous a été recommandé par l'un d'entre eux, n'hésitez toutefois pas à lui envoyer un courriel pour lui dire comment ça s'est passé. Les réseaux professionnels sont complexes et se chevauchent, et vous découvrirez souvent que les personnes que vous rencontrez se connaissent toutes entre elles. Voilà une autre occasion d'envoyer un courriel. Certains de vos contacts utiliseront vos visites pour communiquer ou renouer avec quelqu'un d'autre qui se trouve sur votre liste. Avec le temps, tout le monde connaîtra votre nom et sera au courant de l'intérêt que vous portez à la profession.

Le plus grand avantage de votre réseau est qu'il vous informera des occasions d'emploi. La plupart des emplois (la majorité) ne sont jamais affichés et votre réseau constitue votre seul moyen d'être mis au courant de leur existence. En général, un de vos contacts vous informera si une occasion d'emploi se présente.

Quand cela arrive, vérifiez auprès de votre réseau pour :

- déterminer si le poste vous convient ;

- obtenir des conseils sur la meilleure façon de postuler;

- découvrir quels sont les problèmes principaux auxquels fait face l'organisation ;

- savoir qui embauche et s'il est approprié de communiquer avec cette personne. Établir un premier contact, au lieu de vous contenter de passer par le processus d'embauche, multiplie grandement vos chances de succès.

\section{Le réseautage quand on est aux études}

Ne vous attendez pas à voir apparaître un réseau complètement fonctionnel du jour au lendemain. À moins d'avoir des contacts en lien direct avec votre domaine de prédilection, il faudra plusieurs mois avant que votre réseau ne porte ses fruits, par des conseils et 


\section{Créer et dynamiser votre réseau}

Beaucoup d'associations professionnelles accueillent des membres étudiants. Par exemple, I'Institut d'administration publique du Canada offre une adhésion destinée aux étudiants qui souhaitent travailler pour le gouvernement. Pour la modique somme de 55 \$, les étudiants ont accès aux publications de l'Institut et sont invités à des événements professionnels dont certains sont organisés expressément pour eux et pour les nouveaux professionnels.

L'adhésion à une association professionnelle en tant qu'étudiant est un excellent moyen de commencer à créer un réseau et d'apprendre ailleurs qu'en salle de classe. Vous ne devrez investir que très peu de votre argent et de votre temps, mais vous pourriez être grandement récompensé.

Vous pourriez également élargir votre réseau en écrivant des articles dans les journaux locaux et les publications en ligne, lesquels sont toujours à la recherche de nouveau matériel, soumis au moment opportun (en particulier si ce matériel est gratuit!). Vous pourriez composer un court article (300 à 500 mots) sur un thème qui vous intéresse ou sur un sujet d'actualité dans votre domaine d'études ou d'activités parascolaires. Incorporez-y des citations de vos professeurs ou de professionnels provenant de votre réseau de contacts pour que votre histoire soit encore plus publiable.

La rédaction d'un article vous fera remarquer, vous donnera une raison légitime de communiquer avec les membres de votre réseau et vous pourrez leur laisser ce document.

des tuyaux sur les occasions d'emploi. Ceux qui ont changé de carrière affirment en effet qu'il leur a fallu de quatre à six mois pour se construire un réseau dans leur nouveau domaine.

La meilleure stratégie est de commencer alors que vous êtes aux études. Demandez à vos professeurs ou à vos assistants de recherche de vous donner des noms de contacts (comme d'anciens étudiants). Sur le campus, assistez à des présentations sur des sujets qui vous intéressent et présentez-vous au conférencier ou à la conférencière. S'il y a des conférenciers dans vos cours, allez les rencontrer et discuter avec eux après leur présentation. Demandez-leur une carte professionnelle et communiquez avec eux plus tard. Ils ne se 
souviendront peut-être pas de votre nom, mais ils seront heureux qu'un étudiant se souvienne d'eux et veuille plus d'informations.

Une recherche pour une dissertation ou une présentation représente une occasion idéale d'établir de nouveaux contacts et d'obtenir de l'information. Les programmes avec stages sont d'excellents moyens de créer un réseau. Si vous attendez l'obtention de votre diplôme pour commencer à chercher des occasions d'emploi, vous avez gaspillé toutes les chances que vous auriez eues pour ce faire pendant vos années d'études. De plus, si vous commencez tôt et entretenez votre réseau pendant des années au lieu de quelques mois, il sera basé sur de véritables relations et sera donc plus efficace.

\section{L'usage inapproprié de l'entrevue d'information}

Il est possible d'abuser de toute bonne chose. Certains tenteront hypocritement d'utiliser les entrevues d'information pour chercher un emploi. Sachez que les employeurs et les professionnels de votre domaine de prédilection détestent qu'on les utilise de la sorte. Il est donc essentiel que vous cherchiez réellement à obtenir de l'information sur une carrière professionnelle qui vous intéresse vraiment. Il est tout aussi important de ne pas utiliser l'entrevue comme un moyen de faire chercher les autres à votre place pour vous trouver du travail. Finalement, n'utilisez jamais l'entrevue comme un moyen détourné d'avoir accès à quelqu'un qui, croyez-vous, pourrait vous donner un emploi.

\section{Réseautage et mentorat}

Même si votre carrière progresse bien, vous aurez toujours besoin de l'appui de votre réseau. Vous devez donc l'entretenir. Comme le disent les conseillers en carrière, vous devez le garder au chaud. Pour certains, ce sera facile, mais pour d'autres, ce sera une tâche qui devra être planifiée.

Rappelez-vous qu'un réseau a comme base la réciprocité. Attendez-vous donc à ce que des étudiants vous sollicitent pour obtenir des entrevues $d$ 'information. Traitez-les bien et rappelez-vous que c'est donnant, donnant. Ils vous aideront à garder votre réseau bien au chaud en communiquant avec les gens que vous leur recommandez. 


\section{La vraie entrevue}

Dans son livre, De quelle couleur est votre parachute? : Un guide pratique pour les chercheurs d'emploi et les personnes en transition de carrière (alias le meilleur livre qui soit sur la recherche d'emploi!), Richard Bolles estime que $98 \%$ des chercheurs d'emploi se rendent à leur entrevue comme des moutons à l'abattoir. Ces moutons ne réalisent pas que les entrevues ne sont qu'un moyen d'évaluer s'ils ont des atomes crochus avec l'emploi et l'environnement de travail.

Cette évaluation mutuelle se compare à celle que vous faites quand vous essayez de trouver l'âme sœur. Vous devez être vousmême, mais à votre meilleur. Vous ne révéleriez jamais tout de vousmême à la première rencontre, n'est-ce pas? Vous écouteriez autant que vous parleriez, non? Vous voudriez vous assurer que cette première rencontre ne soit pas la dernière? Il en va de même dans le cas d'une entrevue.

Les entrevues sont régies par certaines conventions auxquelles vous devez vous soumettre :

- Portez une tenue professionnelle. Peu importe si vous n'êtes pas à l'aise. Ceux qui vous font passer l'entrevue s'attendent à ce que vous soyez habillé de manière appropriée à une entrevue professionnelle;

- Arrivez environ dix minutes à l'avance. Cela démontre que vous savez planifier votre temps et que vous respectez celui de ceux qui vous font passer l'entrevue ;

- Si on vous offre du thé ou du café, répondez «Non, merci». Siroter un café pendant une entrevue n'est pas professionnel ;

- Ouvrez votre bloc-notes et soyez prêt à prendre des notes. Vous indiquez ainsi que vous écoutez activement. Cela vous aidera également à répondre aux questions. Notez les noms de ceux qui vous font passer l'entrevue. Vous pourrez ainsi vous en souvenir pendant et après l'entrevue ;

- Ceux qui ont l'habitude de faire ce genre d'exercice vous parleront peut-être d'abord de tout et de rien pour vous mettre à l'aise. Sachez reconnaître de quoi il s'agit et ne vous laissez pas aller à relaxer. Souvenez-vous qu'une interaction complexe est sur le point de se produire. Encore une fois, pensez à une première date et vous ne serez pas loin de la réalité ;

- La plupart des questions d'entrevue comportent un sens caché. Nous traiterons de cela plus tard. Plusieurs d'entre 
elles cherchent à découvrir des problèmes dans votre passé ou votre personnalité, lesquels vous rendraient inadéquat pour le poste ;

- Ceux qui font passer l'entrevue sont à l'affût de tout signe qui indiquerait que vous manquez de motivation, d'enthousiasme, d'esprit d'équipe ou d'éthique, que vous êtes paresseux, arrogant, irresponsable, geignard, indiscret, ou que vous avez du mal à accepter l'autorité. Vous devez donc éviter de donner des réponses qui indiqueraient un tant soit peu que vous possédez ces traits de caractère indésirables.

Si vous regardez les choses du bon côté, l'employeur cherche aussi à déterminer si vous pourrez vous intégrer, apprendre et utiliser les compétences que vous avez acquises au cours de vos études. Tout ce que vous avez à faire est de fournir le plus d'informations possible pour prouver que vous êtes mature et responsable, un joueur d'équipe et que vous possédez d'excellentes habiletés en communication ainsi que de bonnes aptitudes critiques.

Regardez vos interlocuteurs dans les yeux. Souriez, mais ne faites pas de blagues. N'essayez pas d'être ironique, sarcastique, de faire des mots d'esprit ou quoi que ce soit d'autre qui pourrait miner votre professionnalisme. Beaucoup de ces comportements indiquent une personnalité potentiellement problématique.

Apportez quelques copies de votre CV et de votre lettre d'accompagnement, vos meilleures dissertations, vos relevés de notes et tout autre document scolaire ou provenant de votre emploi actuel ou antérieur. Vous n'en aurez peut-être pas besoin, mais le fait d'être en mesure de les produire sur demande pour montrer ce que vous avez accompli vous donnera confiance. Si on ne vous les demande pas, vous pouvez toujours offrir d'en laisser une copie, si c'est approprié. C'est un geste qui pourrait vous permettre de vous distinguer des autres candidats, car vous laisserez un souvenir de vous.

Généralement, vers la fin de l'entrevue, on vous demandera si vous avez des questions. Si vous n'en avez pas, vous avez gaspillé une merveilleuse occasion d'exposer votre connaissance de l'organisation et l'intérêt que vous y portez. Vous avez probablement aussi donné l'impression que vous êtes trop passif.

Que ce soit évident ou non, votre sang-froid, votre intelligence et votre professionnalisme sont toujours en observation. Certaines entrevues sont des interrogatoires en règle, alors que d'autres semblent faciles comme tout. Ne vous faites pas avoir par l'une ou l'autre. 
Gardez votre calme et ayez une attitude positive, même si vous croyez que la personne qui vous pose les questions vous déteste. Vous pourriez être agréablement surpris.

De même, ce n'est pas parce que l'entrevue a été une expérience agréable que c'est dans la poche. Soyez réaliste. Ce n'est pas parce que la première date s'est bien déroulée qu'on vous fera une demande en mariage ou qu'il y en aura même une deuxième.

Les émissions de télévision comme The Bachelor, The Bachelorette ou Occupation double peuvent être très informatives. Ce n'est pas toujours les concurrents ou les concurrentes les plus gentils ou les plus beaux qui gagnent. Parfois, le jeu n'en vaut même pas la chandelle, comme l'ont découvert certains participants par la suite. En même temps, il est facile d'y repérer ceux qui ruinent leurs chances en révélant trop d'eux-mêmes trop tôt, en ayant besoin de trop d'attention ou en agissant de manière à laisser croire qu'ils seraient plus à l'aise dans les bras noués d'une camisole de force!

Ceux qui font passer l'entrevue ont habituellement un horaire très serré. Ne soyez donc pas étonné si celle-ci se termine de manière un peu abrupte. Vous vous sentirez peut-être rejeté si ça se produit, mais il est très important de ne pas vous laisser déconcerter, de vous lever rapidement, de serrer la main de tous, de les remercier de manière courtoise et brève, et de quitter la pièce. N'ajoutez pas de dernières remarques ni n'affirmez à quel point vous aimez l'organisation ou quoi que ce soit d'autre. C'est fini. Circulez!

\section{Les exceptions}

Évidemment, il y a des exceptions à toutes les règles. Il est possible que ceux qui vous font passer l'entrevue ne se conforment pas du tout à ces rituels. Ils pourraient décider de vous embaucher justement parce que vous étiez sarcastique, que vous portiez des Converse ou que vous aviez du chien. Ne comptez pas trop là-dessus, par contre. Si vous ne vous pliez pas aux règles, vous signalez que vous pourriez représenter un problème. La dernière chose qu'un gestionnaire veut, c'est un problème potentiel.

Pensez-y. Le recrutement d'un diplômé coûte beaucoup de temps et d'argent à une organisation. Il en coûte toutefois encore plus de se débarrasser de quelqu'un qui ne s'intègre pas. C'est pourquoi les employeurs ne prennent presque jamais de risques quand ils sélectionnent un nouvel employé. 
En général, peu importe le contexte de l'entrevue, il est préférable de se garder une petite gêne. Ne révélez pas d'un coup toute votre personnalité. L'entrevue n'est ni le temps ni l'endroit de vous mettre à nu.

\section{Le courriel ou la lettre de remerciement}

Toutes choses étant égales par ailleurs, ceux qui envoient une note ou un courriel de remerciement après l'entrevue ont de bien meilleures chances d'obtenir le poste que ceux qui ne le font pas. De plus, c'est l'occasion d'aborder des sujets qui ne l'ont pas été pendant l'entrevue.

Une lettre ou un courriel de remerciement ne devraient pas comporter plus de deux ou trois courts paragraphes. On les lit rarement, mais ça ne veut pas dire qu'ils sont inutiles. On se souviendra que vous avez pris la peine de les rédiger et de les envoyer. Vous trouverez un exemple de lettre de remerciement à l'annexe 3, à la fin de ce chapitre.

\section{Décoder les questions d'entrevue}

Comme dans le cas d'un examen, pour réussir une entrevue, vous devez en comprendre les questions. Ci-dessous, nous décodons pour vous plusieurs questions d'entrevue:

\section{Parlez-nous de vous.}

Traduction: Voici un peu de corde. Voulez-vous vous la passer au cou?

Explication: En posant cette question, on cherche à savoir si vous risquez de poser problème. On s'interroge également sur l'image que vous avez de vous-même. Qu'est-ce que vous pensez de vous?

Solution: Vous devez indiquer que vous êtes quelqu'un de fiable, avec un bon dossier d'emploi, qui a bien profité de ses études, et non pas raconter votre vie. Vous pouvez répondre à cette question de manière constructive en citant des commentaires positifs qu'ont eus à votre sujet vos employeurs, vos professeurs ou vos références.

Durée: Maximum deux minutes.

\section{Pourquoi avez-vous postulé cet emploi ?}

Variante: Quel genre d'emploi cherchez-vous?

Traduction: Montrez-nous comment vous correspondez à ce que nous cherchons. 
Explication: De toute évidence, ceux qui vous font passer l'entrevue en savent beaucoup plus que vous sur le poste à pourvoir. On vous demande d'expliquer ce que vous voulez tirer de cet emploi avant même que vous n'en connaissiez tous les détails. Si votre description ne correspond pas à la leur, vous pourriez être éliminé.

Solution: Si vous savez exactement ce qu'ils cherchent (grâce à votre réseau ou parce que vous avez étudié l'organisation), prouvez-leur qu'ils ont trouvé chaussure à leur pied. Si vous ne le savez pas, renvoyezleur la question et demandez-leur plus de détails sur le travail comme tel. Dites quelque chose comme: «Pourriez-vous me donner quelques détails sur ce que ce poste implique? Ça me permettrait de vous montrer comment je réponds effectivement aux exigences de celui-ci.»

Durée : Jusqu'à dix minutes. C'est le cœur de l'entrevue.

\section{Quelle expérience/expertise avez-vous de ce genre de travail ?}

Variante: Qu'est-ce qui vous fait croire que vous pourriez accomplir ce travail?

Traduction: Vous ne semblez pas avoir beaucoup d'expérience. Vous ne pouvez probablement pas faire ce travail.

Explication: Les candidats qui entrent sur le marché du travail professionnel et qui n'ont pas encore l'expérience que beaucoup d'employeurs recherchent peuvent avoir la certitude qu'on leur posera cette question.

Solution: Ne vous excusez pas de ne pas avoir d'expérience. Ne dites pas non plus que vous êtes motivé à apprendre ce que vous ne savez pas. Vous donnerez l'impression que vous manquez d'autonomie et que vous aurez besoin d'attention. Prouvez-leur que vous avez des compétences que vous pouvez transférer à votre emploi, y compris votre professionnalisme. Finalement, rappelez-leur que vous avez acquis la maîtrise de plusieurs choses dans le passé et que vous comptez faire de même dans le futur.

Durée: Jusqu'à dix minutes. C'est le moment de vous mettre en valeur et de les convaincre que vous êtes le candidat idéal.

\section{Pouvez-vous nous décrire comment vous avez fait face à une situation difficile impliquant d'autres personnes?}

Variante: Vous entendez-vous bien avec vos employeurs et vos collègues?

Traduction: Prouvez-nous que ce n'est pas difficile de s'entendre avec vous. 
Explication: Tous les postes se fondent sur la collaboration et le travail d'équipe. C'est pourquoi il est de loin préférable d'embaucher quelqu'un qui est capable de travailler avec les autres, surtout dans le cas d'un poste de base, que quelqu'un de brillant, mais avec qui il est difficile de s'entendre. Si vos interlocuteurs soupçonnent que vous êtes du genre à créer des problèmes, vous serez rayé de la liste. C'est une question qui peut comporter plusieurs niveaux, lesquels deviendront de plus en plus évidents à mesure que l'entrevue progresse. On cherchera également à déterminer si vous avez tendance à critiquer vos employeurs ou vos collègues. Si vous le faites, vous vous tirez dans le pied. Si vous le faites pendant une entrevue, alors que vous vous comportez de manière exemplaire, vous indiquez que vous risquez de le faire à d'autres moments et encore plus fréquemment au travail.

Ceux qui vous font passer l'entrevue vous pousseront peut-être à parler d'un conflit sérieux que vous avez eu avec un employeur, un collègue ou un professeur. Puisque pratiquement tout le monde sur la planète a déjà eu au moins une relation interpersonnelle conflictuelle, vous ne pouvez pas éviter ce piège.

Solution: Relatez une situation de travail où vous avez agi en adulte et de manière à ce que les autres se rangent de votre côté ou à désamorcer un conflit. Mettez toujours l'accent sur votre grande capacité à résoudre les conflits et sur les leçons que vous avez tirées de situations ou de personnes difficiles.

Si on vous demande plus de détails, donnez-en. Souvenez-vous seulement de les présenter de façon mature et comme des expériences d'apprentissage. Trouvez le moyen de dire des choses positives sur les personnes impliquées. Ne dénigrez jamais les autres ou leurs actions, même s'ils sont les individus les plus détestables du monde. Cela vous ferait mal paraître. Votre objectif est de montrer que vous ne vous laissez pas atteindre par des situations somme toute insignifiantes, même si, de toute évidence, vous n'étiez pas dans votre tort. Ça nous est tous arrivé. Ce qui compte, c'est la façon dont nous faisons face à ce genre de situations ou de personnes.

Durée: De dix à quinze minutes, selon le nombre de questions qu'on vous posera.

\section{Pourquoi y a-t-il une interruption dans votre historique d'emploi/ d'études?}

Variante: Pourquoi y a-t-il un intervalle d'un an entre la fin de vos études secondaires et le début de vos études postsecondaires? 
Pourquoi avez-vous pris cinq ans pour terminer un programme de quatre ans ? Qu'avez-vous fait pendant l'année qui n'est pas mentionnée dans votre $\mathrm{CV}$ ?

Traduction: Avez-vous tendance à abandonner, à échouer ou à perdre votre temps?

Explication: Les interruptions dans l'historique d'emploi étaient autrefois les plus grosses taches sur un CV. De nos jours, les employeurs s'attendent à davantage de transitions dans le cheminement de carrière des individus et perçoivent le changement plus positivement qu'auparavant. Les interruptions leur donnent tout de même l'occasion de sonder votre parcours.

Solution: Vous devez démontrer que vous êtes sérieux par rapport à votre travail. Et c'est pour ça, parce que vous êtes sérieux, que vous n'avez ni travaillé ni étudié pendant un moment; ce temps vous a permis de vous préparer à relever des défis ou à mettre à jour vos compétences. Vous avez utilisé cette interruption pour devenir une meilleure personne et un meilleur employé. Rassurez vos interlocuteurs : dites-leur que vous êtes sérieux au sujet de cet emploi et prêt à vous engager pour au moins cinq ans.

Durée: Maximum cinq minutes.

\section{Donnez-nous un exemple de problème que vous avez résolu dans} la vie, à l'école ou au travail.

Variante: Avez-vous déjà fait face à un problème sérieux ? Comment l'avez-vous résolu?

Traduction: Prouvez-nous que vous pouvez résoudre des problèmes.

Explication: Il s'agit d'une question positive. On cherche à savoir si vous avez acquis les compétences essentielles dont nous avons traité dans ce livre. Les employeurs veulent embaucher des gens qui peuvent détecter des petits problèmes avant qu'ils ne deviennent de gros maux de tête et trouver des solutions créatives. Une des plus grandes déceptions d'un employeur est un employé passif qui fait bien son travail, mais qui ne contribue pas à la survie ou au succès de l'organisation. Le but de toute personne qui fait passer une entrevue est de trouver celui ou celle qui résoudra les problèmes sans ajouter aux difficultés que traverse l'organisation.

Solution: Il ne vous sera pas possible de bien répondre à cette question si vous n'avez pas développé vos compétences essentielles. Il est facile de repérer les réponses inventées de toutes pièces et les creuser un peu plus ne fera qu'exposer davantage vos faiblesses fondamentales. Une 
bonne réponse requiert de la préparation avant l'entrevue. Réfléchissez à des problèmes et à des difficultés que vous avez eus dans le passé et à la manière dont vous leur avez fait face. Si vous aviez pu mieux les aborder, dites-le et expliquez comment vous y répondriez aujourd'hui.

Vous pouvez utiliser des exemples qui viennent de vos études pour répondre à cette question (et à d'autres). Vous faisiez peutêtre partie d'un groupe pour un projet scolaire dans lequel un des membres ne faisait pas sa part de travail. Comment avez-vous fait face à ce problème ? Comment aborderiez-vous une situation similaire au travail ?

Ce que vous devez faire ici, c'est prouver que vous pouvez à la fois cerner les problèmes et travailler avec logique et créativité à les résoudre. Vous devez également démontrer que, pour ce faire, vous pouvez penser latéralement et verticalement. Si certaines de vos solutions les plus originales étaient le résultat de votre travail avec les autres, tant mieux!

La capacité à marier créativité et collaboration à la résolution de problèmes ne peut qu'impressionner vos interlocuteurs.

Durée: Jusqu'à dix minutes.

\section{Quelle est votre plus grande faiblesse?}

Variante: Selon vous, quelles qualités personnelles devriez-vous améliorer pour être une meilleure personne ou un meilleur employé ?

Traduction: Donnez-nous des raisons de rejeter sur le champ votre candidature.

Explication: Cette question arrive habituellement vers la fin de l'entrevue, alors que vous êtes un peu fatigué ou que vous vous sentez à l'aise au point d'être un peu arrogant. C'est un piège très dangereux qui vise uniquement à vous éliminer comme candidat. C'est la question qui tue, à moins d'y être bien préparé.

Les candidats honnêtes, mais qui manquent d'expérience, se font souvent avoir par cette question, surtout s'ils ont l'impression que l'entrevue s'est bien déroulée et qu'ils ont des atomes crochus avec leur interlocuteur. Peu importe à quel point la personne qui se trouve en face de vous est gentille, il s'agit d'une question provenant tout droit du répertoire classique des questions d'entrevue et elle vise à repérer les problèmes. L'honnêteté mal informée ne constitue pas ici la meilleure approche.

Solution: Heureusement, personne n'est parfait. Donnez un exemple de faiblesse qui compte également comme une force. Dites 
quelque chose comme: "J'ai tendance à travailler un peu trop fort quand je travaille sur un projet intéressant» et vous ne devriez pas être éliminé. Vous pouvez même tirer avantage d'une faiblesse si vous dites: "Je suis le genre de personne qui a de l'initiative et qui aime aller au-delà de ce qu'on attend d'elle. Ça signifie que je peux trouver occasionnellement difficile d'être supervisé de trop près. » Si vous obtenez l'emploi, votre superviseur vous laissera probablement plus de liberté que prévu.

Durée: Maximum cinq minutes.

\section{Où vous voyez-vous dans cinq ans?}

Variante: Comment envisagez-vous le développement de votre carrière?

Traduction: Êtes-vous seulement à la recherche d'un salaire ou voulez-vous une carrière qui veuille dire quelque chose pour vous?

Explication: Les employeurs veulent savoir si vous valez l'investissement. Progresserez-vous avec l'organisation? Voudrez-vous grimper les échelons? C'est ce qu'ils souhaitent.

La durée de cinq ans signifie aussi quelque chose. Au sein de beaucoup de professions, il est entendu que les gens sont susceptibles de changer d'organisation après cinq ans, afin de progresser dans leur carrière. Il est parfois plus facile de grimper les échelons en allant ailleurs qu'en restant au même endroit. Vous devez donc savoir cela, si ce n'est que parce que vos interlocuteurs en sont aussi conscients.

Solution: Démontrez que vous avez une dose raisonnable d'ambition, ce qui signifie que vous devez avoir fait un peu de recherches sur votre carrière et sa trajectoire possible. Vous voulez signifier que vous vous développez bien dans un environnement qui comporte des défis et qui offre des chances d'avancement, sans toutefois donner l'impression que vous bouleverserez le bureau. Vous voulez avoir l'air de quelqu'un qui évoluera et qui se développera au cours de sa carrière.

Dans cinq ans, vous voulez avoir amélioré vos habiletés professionnelles à un point tel que votre employeur n'aura d'autre choix que de reconnaître votre évolution et vous promouvoir à un poste qui vous convient. Vous sous-entendez ainsi que vous irez voir ailleurs pour trouver de quoi vous motiver si on ne vous l'offre pas au sein de votre organisation. Vous ne devriez toutefois jamais être explicite à ce sujet, au risque de mettre en doute votre engagement envers l'organisation.

Durée: Maximum cinq minutes. 


\section{9. À quel salaire vous attendez-vous?}

Variante: Quel était votre salaire au dernier emploi que vous avez occupé?

Traduction: Avons-nous les moyens de vous embaucher?

Explication: Pendant l'entrevue, votre interlocuteur a toujours à l'esprit ce que vous risquez de coûter, en particulier si vous êtes un candidat hors pair, avec des diplômes spécialisés et de l'expérience. Même s'il n'est pas très professionnel de la part de ceux qui font passer l'entrevue de poser des questions au sujet du salaire, ils essaieront souvent de découvrir à quoi vous vous attendez afin d'être plus en mesure de décider entre deux candidats équivalents.

Ils savent aussi que vous serez dans une meilleure posture pour négocier votre salaire quand ils vous auront offert le poste. Si vous vous laissez prendre à accepter un salaire maintenant au lieu d'attendre d'avoir un contrat en main, vous leur simplifiez énormément la vie.

Solution: Vous ne savez jamais quel genre de pression vous devrez subir en entrevue. C'est pourquoi c'est une bonne idée d'avoir en tête une fourchette salariale basée sur la moyenne statistique pour votre profession. Il est cependant important de ne pas négocier de salaire, à moins qu'on ne vous torde le bras.

Expliquez simplement que vous ne pouvez pas discuter de salaire avant qu'on ne vous ait fait une offre d'emploi formelle et que vous ne la considériez sérieusement. En ce moment, vous préférez vous concentrer sur le plus important, c'est-à-dire déterminer si vos compétences correspondent à leurs besoins.

Quand on vous offrira le poste, vous entamerez les négociations salariales et serez en mesure d'obtenir le salaire le plus élevé possible pour vous. Dès qu'ils auront fait leur choix, ils ne voudront plus, en effet, recommencer le processus d'entrevue. Soyez raisonnable, mais rappelez-vous également qu'il est plus facile d'obtenir un salaire plus élevé au moment du recrutement ou à l'embauche que si vous êtes déjà en poste.

Durée: Normalement, vous devriez répondre à cette question en moins d'une minute, étant donné que vous ne voulez pas vous engager maintenant.

\section{Avez-vous des questions pour nous?}

Variante: Y a-t-il quoi que ce soit d'autre que vous souhaitez nous dire? 
Traduction: Voici votre chance de vous faire une idée à notre sujet.

Explication: L'ensemble de l'entrevue est structuré autour de leurs questions et de vos réponses. Un candidat intelligent aura compilé une liste de questions avant l'entrevue et les posera au moment approprié. En règle générale, une bonne entrevue permet une répartition équitable du temps de parole entre le candidat et ses interlocuteurs. Chacun fait la cour à l'autre et essaie de déterminer s'il y aura entente.

Selon la nature de l'entrevue, il est possible que vous ne puissiez pas poser de questions en cours de route et que vous deviez attendre à la fin de la séance (si l'horaire d'entrevue est très chargé ou si plus d'une personne fait passer l'entrevue).

Même s'ils ne sont pas particulièrement intéressés par vos questions, vos interlocuteurs s'attendront à ce que vous en ayez quelquesunes. Si vous n'en avez pas, vous signifiez que vous n'êtes pas autonome, que l'emploi ne vous intéresse pas ou que vous n'avez pas fait suffisamment de recherches sur le poste et l'organisation.

Solution: Prouvez que vous avez fait vos recherches et posez des questions sur le travail ou sur l'organisation. Il est toujours très efficace de relever une problématique ou un problème auquel l'organisation, l'industrie ou la profession fait face et de demander l'opinion de vos interlocuteurs sur le sujet. Profitez de l'occasion pour faire étalage de vos connaissances et de votre intérêt.

Vous ne devriez pas être le sujet de vos questions. Vous pouvez demander des clarifications sur certains aspects du poste ou encore préciser une de vos réponses, mais il est plus efficace de démontrer votre intérêt envers eux plutôt que de parler de vos besoins et de vos désirs. C'est une approche qui fonctionne bien tant pour ceux qui sont à la recherche de l'âme sœur que ceux qui cherchent un emploi.

Durée: Cela dépend, mais préparez au moins dix minutes de questions, même si les réponses sont courtes. Voici les dix questions à poser lors d'une entrevue formelle :

- Pourriez-vous me décrire une journée typique dans ce poste?

- Quels sont les trois objectifs principaux de votre organisation pour l'année qui vient?

- Quelles sont les plus grandes difficultés du poste?

- Quels sont les plus grands défis auxquels doit faire face votre organisation?

- Quelles sont les possibilités d'avancement pour ceux qui excellent dans ce poste?

- Quel est votre style ou votre philosophie de gestion? 
- À quel niveau situez-vous ce poste par rapport à l'ensemble des postes de l'organisation?

- Quel genre de personnes ont le plus de succès au sein de votre organisation?

- Quel genre de personnes n'ont pas réussi au sein de votre organisation?

- Pouvez-vous me décrire votre employé idéal?

\section{Les services et les entrevues sur le campus}

Votre collège, votre cégep ou votre université a fort probablement un service ou un bureau de préparation à la carrière. Il serait judicieux de votre part de le visiter pour voir ce qu'il a à vous offrir. Peu importe que vous y trouviez peu ou beaucoup, l'important, c'est de vérifier. Il reste que trouver votre emploi idéal est un projet que vous devez réaliser vous-même. Votre établissement d'enseignement ou votre programme vous donnera peut-être les outils pour le faire, mais c'est à vous de les manipuler pour arriver à vos fins.

Certains employeurs font passer des entrevues sur le campus, lesquelles sont souvent coordonnées par le centre des carrières ou le bureau de placement. Vous pouvez vous y inscrire afin de mettre à jour votre $\mathrm{CV}$ et, si vous avez une entrevue, développer vos habiletés d'entrevue.

\section{Les entrevues dans de petites entreprises}

L'ensemble des conseils ci-dessus vaut pour de grandes organisations qui possèdent du personnel formé en ressources humaines. Il n'en demeure pas moins que, de nos jours, beaucoup de petites entreprises créent de l'emploi. Dans ce cas, la personne qui fait passer l'entrevue sera généralement l'employeur, ce qui a des avantages et des inconvénients pour celui qui se cherche un emploi.

Côté avantages, vous passerez probablement l'entrevue en présence de la personne qui embauche et qui prendra rapidement une décision. Par contre, elle n'aura pas de formation en technique d'entrevue. Les professionnels en ressources humaines possèdent, en effet, une expertise considérable et sont habiles pour mettre les gens à l'aise et pour découvrir leur potentiel.

Ceux qui ne possèdent pas de formation en ce sens sont souvent tendus et mal à l'aise lors de l'entrevue. Il peut arriver qu'ils soient insensibles, voire parfois insultants, quoique involontairement. Dans 
ce genre de situation, plusieurs des rituels dont nous avons traité plus haut ne s'appliqueront pas et vous devriez changer votre stratégie en conséquence.

Voici ce dont vous devez vous rappeler au sujet des entrevues menées par le propriétaire d'une petite entreprise :

- Il est probablement aussi nerveux que vous ;

- Vous avez la responsabilité de vous assurer qu'il vous pose les bonnes questions;

- Il est essentiel de le mettre à l'aise ;

- Il est encore plus important que jamais de faire un suivi après l'entrevue, puisque cette personne n'aura probablement pas grand-chose sur quoi baser sa décision;

- Ne vous en voulez pas si vous ne décrochez pas l'emploi. Vous vous êtes exercé à passer une entrevue et vous aurez la chance d'en passer beaucoup d'autres si vous êtes à la recherche d'un poste dans une petite entreprise ;

- Essayez d'ajouter l'employeur à votre réseau. Il y a de fortes chances qu'il embauche la mauvaise personne et qu'il cherche bientôt quelqu'un d'autre.

À bien des égards, les petites entreprises sont des environnements idéaux pour votre croissance personnelle, car elles reposent sur moins de bureaucratie et offrent beaucoup d'occasions de développer vos compétences professionnelles.

Les plus petites entreprises n'ont pas les moyens d'investir dans le recrutement et doivent donc davantage se fier au réseautage pour trouver des employés. Elles sont également plus ouvertes aux relations de travail non traditionnelles.

Vous devriez considérer les postes qu'offrent les petites entreprises innovantes. Elles représentent souvent le meilleur endroit où commencer votre carrière et accumuler de l'expérience professionnelle.

\section{Les styles d'entrevue}

Les conseils que nous avons fournis ici fonctionneront dans la plupart des cas. Certaines organisations choisissent toutefois des façons très différentes de mener une entrevue. 


\section{L'approche conversationnelle}

La personne qui fait passer l'entrevue converse ici avec vous au lieu de vous poser des questions directes. Elle vous sonde de manière tellement décontractée et détendue qu'il est facile de vous laisser amadouer et de révéler trop d'informations. La clé ici est de vous rappeler les règles pour bien vous en tirer en entrevue et de toujours demeurer professionnel.

\section{L'approche par consensus}

Puisque le recrutement et la rétention des bons employés coûtent cher, plusieurs organisations font des entrevues en comité, sous prétexte que plusieurs têtes valent mieux qu'une quand vient le moment de sélectionner un nouvel employé. Ces entrevues peuvent être intimidantes, car le candidat se trouve devant plusieurs personnes qui ont parfois des conflits de personnalité.

Dans de telles situations, il vous est impossible de contrôler les variables comme vous pourriez le faire au cours d'une entrevue en tête-à-tête. Le secret est de vous exercer à bien écouter (écoute active !) et de prendre des notes. Cela vous permet de saisir l'implicite de la question qu'untel vous posera, tout en continuant à regarder les autres dans les yeux et à garder un lien avec eux.

Ici, le plus important est de prendre votre temps avant de répondre aux questions. Si quelqu'un pense que vous êtes nerveux et essaie de vous aider, informez-le gentiment que vous réfléchissez à la meilleure façon de répondre à ce qu'on vous a demandé.

Même si les entrevues par consensus semblent stressantes, il est plus facile de bien y faire que dans une entrevue en tête-à-tête avec un interlocuteur expérimenté dans ce genre d'exercice. L'examen y est moins rigoureux et les différences entre les personnes vous donnent l'occasion de porter attention à la dynamique de l'équipe.

\section{L'approche par stress}

Un nouveau style d'entrevue vise à vous mettre dans des situations stressantes. On y parvient en utilisant une variété de techniques grossières, comme faire des affirmations controversées et vous demander d'y répondre immédiatement ou répondre à vos questions par un silence prolongé.

L'efficacité de cette approche est hautement discutable. Elle se fonde sur l'hypothèse suivante: puisque le monde moderne est un 
endroit en constante évolution, les meilleurs employés sont ceux qui arrivent à gérer leur stress. Ce pourrait être une hypothèse valide si ce n'était du fait que l'entrevue par stress est relativement facile à mâ̂triser. La meilleure stratégie pour vous en sortir est de garder votre sang-froid, de rediriger calmement les questions embarrassantes, de désamorcer les affirmations controversées en les reformulant de manière professionnelle et de donner une réponse équilibrée et judicieuse, malgré le ton ou l'agressivité des interlocuteurs.

Étant donné que la raison d'être des entrevues par stress est de déterminer si vous craquez sous la pression, tout ce que vous avez à faire, c'est de demeurer calme.

\section{Les entrevues d'une journée et au restaurant}

Certaines organisations aiment passer une journée complète ou, du moins, prendre un long repas avec des employés potentiels. Selon cette approche, même si les candidats arrivent à cacher leur vraie personnalité pendant une courte entrevue, celle-ci finira bien par refaire surface pendant une journée occupée ou un repas du soir. C'est un raisonnement discutable, mais si vous passez une entrevue du genre, vous devez savoir certaines choses.

Votre estomac est probablement votre pire ennemi. Pendant une entrevue d'une journée, vous devez vous assurer que le sang circule dans votre cerveau plutôt que dans votre ventre. Munissez-vous donc d'une réserve de collations nutritives en cas de fringales. Ne buvez pas d'alcool lors de l'entrevue au restaurant. La seule chose positive de celle-ci est que vous n'aurez pas à payer pour votre repas.

\section{Les deuxième et troisième entrevues}

Les entrevues successives démontrent à quel point les grandes organisations prennent le processus d'embauche au sérieux. Il fut un temps où on se contentait d'une seule rencontre. De nos jours, toutefois, trois entrevues semblent parfois insuffisantes et les candidats pleins d'espoir finissent par faire un tour de montagnes russes d'entrevues qui pourrait durer des semaines.

C'est une raison de plus de vous détendre. Ça ne vaut pas la peine de vous en faire au sujet d'un poste qui nécessite plusieurs entrevues avec une variété de personnes. Ce serait malsain. De plus, l'intervalle entre la première et la troisième entrevue vous donne le 
temps de réfléchir à la nature de l'organisation et de déterminer si elle vous convient.

Les chercheurs d'emploi d'expérience ne mettent jamais tous leurs œufs dans le même panier. Ils postulent plutôt plusieurs emplois à la fois. Ainsi, s'ils n'obtiennent pas l'un d'entre eux, ils peuvent immédiatement se concentrer sur les autres possibilités, au lieu de s'en faire pour ce qu'ils n'ont pas eu.

\section{Les références}

Dans la plupart des professions, vous ne devez pas soumettre vos références au moment de postuler un emploi. C'est inhabituel, voire très peu professionnel, de les exiger à cette étape. On pourrait vous les demander en entrevue, mais la plupart du temps, les employeurs se préoccuperont des références seulement quand un candidat sera vraiment considéré pour le poste. Ils pourraient demander une lettre de recommandation formelle ou se satisfaire de références par courriel.

Il est essentiel de bien choisir vos références, car les employeurs accordent une grande valeur à ce qu'elles révèlent à votre sujet. À une exception près, dont nous discuterons ci-dessous, ne donnez jamais le nom de quelqu'un comme référence sans lui en avoir d'abord demandé la permission. Décrivez-lui également le poste que vous postulez. Assurez-vous que la personne est à l'aise dans son rôle de référence, sinon, trouvez quelqu'un d'autre.

Des situations problématiques (des doutes sur votre loyauté, par exemple) pourraient survenir si vous donnez le nom de votre employeur actuel comme référence. Pour les éviter, vous pouvez fournir le nom, mais demander qu'on communique avec votre patron seulement si on vous considère vraiment très sérieusement pour le poste, et si on vous en informe d'abord. Vous pourrez ainsi parler à votre employeur actuel au moment où on s'apprête à communiquer avec lui.

Les professeurs sont de bonnes références pour les diplômés récents et les étudiants puisqu'on les perçoit comme impartiaux. Si vous vous entendez bien avec un ou deux professeurs, n'hésitez pas à leur demander d'agir comme références pour vous. Rappelez-vous toutefois qu'ils voyagent parfois pour leurs recherches (en particulier l'été) et qu'ils ne peuvent pas nécessairement répondre immédiatement aux demandes de référence. 
En général, on vous demandera de fournir trois références. Optez pour la variété : votre employeur actuel, un professeur, un ancien employeur, quelqu'un avec qui vous avez fait du bénévolat, etc.

La mise en page de vos trois références devrait ressembler aux exemples ci-dessous. Assurez-vous de donner des coordonnées complètes. Vous pouvez également inclure quelques notes au sujet de chaque référence pour aider ceux qui communiqueront avec les personnes référées à déterminer le rôle qu'elles ont joué dans votre vie.

Références pour [insérez votre nom]

Professeur Thomas Klassen

Département de science politique

Université York

4700, rue Keele

Toronto (Ontario) M3J 1P3

Téléphone : 416 736-2100, poste 88828

Courriel : tklassen@yorku.ca

[En 2013-2014, j'ai suivi deux cours dispensés par le professeur Klassen, l'un au sujet des politiques publiques et l'autre, sur les méthodes de recherche. Le professeur Klassen préfère qu'on communique avec lui par courriel.]

Madame Simone Sagesse

Gestionnaire

Département des plaintes

Xxx

$X x x$

Téléphone :

Courriel :

[Je suis actuellement à l'emploi de madame Sagesse.]

Monsieur Markhut Smart

Coordonnateur

Xxx

$X x x$

Xxx

Téléphone:

Courriel : 
[Monsieur Smart dirige le centre communautaire où j'ai fait du bénévolat pendant deux ans.]

\section{Après l'entrevue}

Certains employeurs essaient de garder les candidats en haleine pendant qu'ils prennent une décision ou négocient avec leur premier choix. Il est normal pour eux de penser d'abord à leur propre intérêt, mais il est tout aussi important pour vous de faire de même. S'ils vous demandent, comme il arrive souvent, si vous avez d'autres entrevues pour des postes semblables, vous pouvez le leur dire. S'ils sont raisonnables, ils comprendront. S'ils s'intéressent à vous, cette information les motivera peut-être à prendre une décision plus rapidement.

L'attente peut être longue après une entrevue et elle peut sembler durer une éternité. Il n'y a pas grand-chose à faire à cette étape, excepté continuer votre recherche d'emploi et passer des entrevues. Ça ne vous servira à rien de déprimer.

Vous êtes sur le marché de l'emploi et, comme dans n'importe quel autre marché, qu'il s'agisse d'actions, de charcuteries ou de pétrole, il doit y avoir correspondance entre le vendeur (vous) et l'acheteur (l'employeur). Celle-ci n'aura pas lieu immédiatement et probablement pas la première fois que vous essayerez. Attendez de trouver l'emploi qui correspond à vos intérêts et à vos compétences.

Évidemment, c'est beaucoup plus facile à dire qu'à faire. En attendant les réponses des employeurs, gardez contact avec votre réseau et continuez de chercher. Tout vient à point à qui sait attendre! 


\section{Annexe 1 : Exemples de curriculum vitæ}

Terry Mah

113-320, rue Heathdale

Toronto (Ontario) M7S $6 \mathrm{R} 8$

Téléphone/télécopieur: 416-987-5678

Courriel : t.mah@edu.ca

Site Web: monsiteweb.com

\section{ÉDUCATION}

Baccalauréat en histoire - Université York, Toronto, obtention du diplôme prévue en juin

- Spécialisation en histoire nord-américaine du $x^{e}$ siècle, plus particulièrement sur les ententes d'immigration et d'échanges commerciaux.

- MPC: moyenne générale de $B$; moyenne de $B+$ dans les cours d'histoire et de $A$ - dans les cours de quatrième année.

- J'ai rédigé une dissertation de 18 pages sur les relations commerciales entre le Canada et les États-Unis dans les années 1980 dans un cours de quatrième année, pour laquelle j'ai obtenu une note de $A+$ (le professeur a écrit: «travail fantastique, original, qui démontre une recherche en profondeur $»)$.

Baccalauréat en philosophie des religions - Beijing University, Chine, 2008-2010 - incomplet

- Spécialisation en philosophie bouddhiste de l'Asie de l'Est (Chine, Corée et Japon).

\section{EXPÉRIENCE PROFESSIONNELLE}

Commis de bureau - Kim et associés, experts en comptabilité et en impôts sur le revenu, Toronto

Depuis 2014 (temps plein à l'été et temps partiel pendant l'année scolaire, à raison de six heures par semaine)

- Apprendre les principes et les techniques de comptabilité de base afin de soutenir l'équipe de comptables.

- Aider les clients en répondant à diverses demandes de renseignements comptables et financiers au téléphone, en personne et par courrier électronique - comme la confirmation des détails des 
transactions et la vérification des fichiers de l'Agence du revenu du Canada.

- Assigner les questions et les demandes complexes au bon membre de l'équipe et faire un suivi.

- Garder un environnement de travail propre et encourager une atmosphère professionnelle amicale.

- Faire le classement des documents en formats papier et électronique, et les commandes de fournitures de bureau.

Agent à la réception - Hôtel Delta Chelsea, Toronto

2011-2013 (temps plein, été et temps partiel)

- Régler et confirmer, au départ, les comptes des clients, dont certains ne parlent pas français, souvent sous pression.

- Aider à la formation et à la supervision des nouveaux employés à temps partiel, y compris à l'utilisation par ceux-ci des logiciels de réservations et financiers.

- Traiter les dossiers des clients à l'arrivée et au départ, y compris les clients privilégiés et le personnel des compagnies aériennes au comptoir qui leur était réservé.

Agent de réservation - Utell International (agence de voyages internationaux), succursale de Beijing

2010-2011 (temps plein)

- Réserver des chambres d'hôtel partout dans le monde pour des représentants de commerce chinois, selon des échéanciers très serrés, par courriel et au téléphone.

- Faire le suivi des plaintes des clients et de leur résolution auprès d'hôtels en Europe et en Australie.

\section{Chercheur - China National Tourism Corporation, Beijing} 2009 (été)

- Réaliser de courts sondages en personne auprès de voyageurs internationaux en partance des aéroports de Beijing et de Shanghai.

\section{BÉNÉVOLAT}

\section{Enseignant d'éducation physique - École Sainte-Anne, Toronto}

Depuis 2014 (quatre heures le samedi)

- Préparer des exercices hebdomadaires pour les élèves de quatrième et cinquième année, y compris la démonstration des premiers soins. 
- organiser des soirées aux matches des Maple Leafs de Toronto avec les élèves, y compris la collecte de fonds auprès des parents

\section{Ambassadeur étudiant - Université York}

Depuis 2014 (trois heures par semaine)

- Faire des tournées du campus avec les futurs étudiants et leurs parents.

- Traduire des courriels du mandarin vers l'anglais pour les délégations qui proviennent de Chine.

- Diriger les visiteurs vers les événements sur le campus et leur donner de l'information sur l'Université. 
Alex Menzies

Adresse: 34, avenue Hillcrest, Anytown, MY L4S 2Y1

Courriel: Alex.Menzies@myuni.edu

Téléphone: 816 234-5678

\section{ÉDUCATION}

\section{Baccalauréat en commerce et société \\ Université MY, La Ville, 2014}

Date prévue d'obtention du diplôme: mai 2018

- Moyenne de B en première année (cinq cours, y compris psychologie, histoire et anglais)

- Cheminement en sociologie et en études du travail

\section{EXPÉRIENCE PROFESSIONNELLE}

\section{Tutrice privée (soirs)}

\section{À mon compte, Ma Ville, 2012-2013}

- Tutorat d'élèves de cinquième et de sixième année en anglais, français et mathématiques

- Motivation des élèves en organisant des exercices et des devoirs hebdomadaires, et en leur donnant ensuite des commentaires constructifs

- Suivi des progrès des élèves avec les parents, parfois sur rendez-vous

\section{Superviseure adjointe (temps plein)}

Tim Hortons, L'Autre-Ville, 2011-2012

- Résolution de conflits avec les clients et les employés au sujet de la qualité du service

- Gestion des employés à temps partiel (inexpérimentés et énergiques)

- Développement de relations de loyauté à l'égard des clients en mémorisant leur nom et leurs préférences

\section{Commis aux retours (temps plein)}

\section{Entrepôt Nexten, N'importe-Où, 2010-2012}

- Entrée et collecte de données sur les produits dans une grande base de données électronique

- Inventaire physique quotidien des produits retournés et entrée dans la base de données 
- Emballage et étiquetage des produits pour empêcher les dommages et en assurer un transport sans heurts

\section{COMPÉTENCES EN TECHNOLOGIES DE L'INFORMATION}

- Experte en logiciels variés, y compris Microsoft Word, Microsoft Outlook et Adobe Acrobat

- Compétente dans I'utilisation de feuilles de calcul, y compris celles de Microsoft Excel et de Lotus

- Excellentes compétences en saisie au clavier, 80 mots à la minute

\section{AUTRES HABILETÉS ET RÉALISATIONS}

- Parle couramment le français et peut lire le hindi

- Instructrice certifiée en entraînement de groupe (aérobie) avec expérience en enseignement

- Expérience de bénévolat auprès d'enfants de 8 à 12 ans ayant des problèmes de croissance 
Samantha Pereira

2600, rue White, Vancouver (Colombie-Britannique) V1Z 2X3

Téléphone: 604 234-5678

Courriel: sperreira@ubc.ca

\section{ÉDUCATION}

Baccalauréat en sociologie, Université de la Colombie-Britannique - en voie de terminer la première année d'études

\section{EXPÉRIENCE PROFESSIONNELLE}

\section{CAISSIÈRE Safeway}

16 à 24 heures par semaine, 13 avril 2013-14 décembre 2014

- Offrir un service à la clientèle amical et patient pendant les heures d'affluence, sans faire d'erreurs de transaction

- Connaître avec exactitude 245 codes de produits

- Formation en contrôle des vols, y compris la fraude par carte de crédit et par chèque

\section{ÉDUCATRICE DE LA PETITE ENFANCE travailleuse autonome}

3 jours par semaine, 8 heures par jour, 12 juin 2013-13 septembre 2013

- Prendre en charge deux enfants (âgés de 3 et 5 ans) chez eux

- Préparer des repas nutritifs équilibrés et des collations

- Organiser des activités intérieures et extérieures avec les enfants

CHEF D'UN GROUPE DE PAIRS (bénévolat) Service de travail auprès de la jeunesse de Vancouver, prévention de l'usage de stupéfiants et d'alcool, janvier 2013-13 juin 2013

- Aider à la création, avec les autres bénévoles, de matériel d'enseignement créatif et intéressant en formats papier et électronique

- Accompagner les bénévoles lors de présentations dans des classes de $9^{\mathrm{e}}$ et $10^{\mathrm{e}}$ année d'écoles secondaires

TUTRICE (bénévolat) Club de devoirs de la Bibliothèque municipale de Vancouver, mai 2011-juin 2012

- Aider les nouveaux arrivants de 8 à 11 ans à faire leurs devoirs d'anglais

- Créer du matériel d'apprentissage enrichi pour ceux qui terminaient leurs devoirs 


\section{Annexe 2: Exemple de lettre de présentation}

Montréal, le 28 novembre 2018

À l'attention de Madame Gloria Park

Directrice des prêts

Bibliothèque de Montréal

Ressources humaines

$123,15^{\mathrm{e}}$ Rue, $16^{\mathrm{e}}$ étage

Montréal (Québec) A1B 2C3

\section{Objet : Demande d'emploi}

\section{Madame la Directrice,}

Si vous avez besoin d'un employé ayant des aptitudes hors pair en organisation et en communication avec une variété de personnes dans des situations complexes, j'aimerais avoir la chance de vous rencontrer.

Le curriculum vitæ ci-joint décrit quelqu'un qui a fait deux ans d'études collégiales et qui aime travailler avec des groupes diversifiés et des gens qui proviennent d'horizons différents.

II représente quelqu'un qui porte une attention particulière aux détails, croit au contrôle de qualité et possède une connaissance des processus, trois traits de caractère qui sont nécessaires à quiconque souhaite obtenir un poste d'adjoint aux prêts. J'espère également qu'il décrit quelqu'un que vous aimeriez voir faire partie de votre équipe pour offrir des services de manière efficace et amicale.

Bien que je n'aie pas d'expérience en bibliothèque, je connais très bien l'industrie du livre. Dans mon emploi actuel, je suis, en effet, responsable de l'achat et de la vente de livres. L'été dernier, j'ai fait du bénévolat pendant trois mois, les soirs et les fins de semaine, dans le cadre d'un programme de lecture pour enfants dans un centre communautaire près de chez moi. Je devais trouver du matériel de lecture, en formats papier et numérique, convenant à différents groupes d'âge, non seulement en anglais, mais aussi dans d'autres langues, pour les nouveaux arrivants. 
Je me tiens à votre disposition pour discuter avec vous de la façon dont je pourrais contribuer au bon fonctionnement et à l'ambiance chaleureuse du comptoir de prêts.

J'espère que vous ne verrez pas d'inconvénient à ce que je communique avec vous la semaine prochaine, par courriel ou par téléphone. Veuillez agréer, Madame la Directrice, mes salutations distinguées.

[Signature]

André Dawson

53, croissant Marue

Montréal (Québec) L4S 2 Y1

Téléphone: 514-456-7890

Courriel: dawson@moncollege.edu

p. j. Curriculum vitæ 


\section{Annexe 3: Exemple de lettre de remerciement}

Ottawa, le 15 avril 2018

Sabrina Makhamra

Directrice des programmes d'été

Camps d'été Joyeux

12345, rue Sparks, bureau 800

Ottawa (Ontario) K1N 2A1

Madame la directrice,

Je tiens à vous remercier de votre accueil et de m'avoir accordé du temps hier. J'ai été enchanté d'en apprendre davantage sur les Camps d'été Joyeux.

Je vous suis particulièrement reconnaissant d'avoir pris le temps de me montrer les installations et de me donner plus de détails sur les responsabilités du coordonnateur d'été. Pendant notre conversation, vous avez insisté sur le rôle important du coordonnateur d'été dans la formation des conseillers. Soyez assurée que mon programme d'études et mes emplois antérieurs dans des camps d'été m’ont bien préparé au poste de coordonnateur. L'expérience que j'ai acquise à titre de gestionnaire de personnel adjoint, l'été dernier, au Camp Lakeview, m'a été particulièrement utile sur ce plan.

Je suis emballé à l'idée de pouvoir faire de cet été le meilleur été pour le personnel et les enfants des Camps d'été Joyeux.

Si d'autres informations vous étaient nécessaires, n'hésitez aucunement à communiquer avec moi. Il me fera plaisir de répondre à vos questions.

Dans l'attente d'une réponse de votre part, je vous prie de recevoir, Madame la directrice, mes cordiales salutations.

[Signature]

Bruno Baytes

24, chemin Laurier Ouest

Ottawa (Ontario) L4S 2 Y1

Téléphone : 613-456-7890

Courriel: B.Baytes@uni.edu 


\section{Notes}

1. Voir Gouvernement du Canada, Services aux jeunes, «The Hidden Job Market », 22 mai 2014, http://www.youth.gc.ca/eng/topics/jobs/looking.shtml

2. Business Examiner, « Networking, Not Internet Cruising, Still Lands Most Jobs ", Blogue du Business Examiner, 9 août 2012, http://www.businessexaminer.com/ blog/August-2012/Networking-not-Internet-cruising-still-lands-most-jobs/ 


\title{
Tirer profit des médias sociaux
}

\author{
«En ces temps de connexions permanentes \\ et instantanées, quand on fait un retour sur \\ soi on ne se demande plus «Qui suis-je?» \\ mais «Que suis-je en train de faire?»- \\ Tom Chatfield, Comment mieux viore à l'ère \\ numérique
}

T e moins qu'on puisse dire, c'est que rien n'a autant brouillé la rela$\mathcal{L}$ tion entre le soi privé et le soi public qu'Internet. Auparavant, le
domaine privé possédait un caractère sacré que les gouvernements, les employeurs, les professions et les collègues ne cherchaient pas à envahir. En grande partie à cause des médias sociaux, le privé est peu à peu devenu public.

Nous suggérons que vous saisissiez à bras-le-corps les problèmes et les occasions que présentent les médias sociaux. Vous ne serez jamais aussi efficace que vous le pourriez en tant qu'étudiant ou employé, ou même en tant qu'ami ou amoureux, si vous ne profitez pas de ce qu'ont à offrir les médias sociaux.

Nous entendons par médias sociaux la création et l'échange d'idées et d'informations sur Internet : Facebook, Twitter, Tumblr, les wikis, les blogues, YouTube, les mondes virtuels de jeux et beaucoup d'autres. Nous connaissons tous des gens qui semblent se satisfaire de laisser définir par Facebook, Twitter, Instagram, les simulations par 
ordinateurs et les tendances Web, une partie de qui ils sont et de ce qu'ils veulent dans la vie. Ces individus ne font pas de bons étudiants, de bons employés ou de bons amis. Ils ne comprennent pas la différence entre l'utilisation d'outils et de techniques, et le fait d'être l'outil pour quelqu'un ou quelque chose d'autre.

Une stratégie sensée est de profiter des aspects utiles d'un monde sans frontières, sans se laisser envahir. Utilisez les communications sur Internet pour vous développer une identité unique et authentique. Faites vos propres choix.

Le professeur Dwyer est une sorte de béotien en technologies. Il n’a jamais eu de téléphone cellulaire. Il n'est pas sur Facebook et il déteste Twitter, mais il ne peut pas échapper complètement à Internet. Ses étudiants préfèrent communiquer avec lui par courriel. Son université insiste pour qu'il ait une présence en ligne. Son enseignement exige le recours à des pages Web ; au moins, il a un certain contrôle sur l'information qui en provient.

\section{Les cinq règles d'or des médias sociaux}

Internet émet à notre sujet énormément de signes et de signaux, et nous n'avons que très peu de contrôle sur ceux-ci. Tout ce que nous disons ou faisons constitue pour nous de l'information potentiellement dommageable. Par exemple, si vous allez sur le site Web ratemyprofessor.com, vous apprendrez toutes sortes de choses sur le professeur Dwyer qu'il préférerait que vous ne sachiez pas à son sujet. Dans plusieurs années, le professeur Dwyer sera mort et enterré, certes, mais les commentaires de ses étudiants au sujet de son intelligence, de son enseignement et de son apparence risquent d'être toujours bien vivants sur Internet - et à la portée de quiconque veut les lire.

Le professeur Dwyer est vieux (65 ans) et cet âge vénérable lui donne le privilège d'ignorer ce qu'on dit de lui sur Internet. Vous n'êtes vraisemblablement pas aussi vieux que lui (nos condoléances, le cas échéant), ce qui signifie que, pour modeler votre carrière d'étudiant et planifier celle qui viendra par la suite, vous devez prendre en considération les possibilités et les problèmes que présentent Internet et les médias sociaux. La première règle, et la plus importante, au sujet des médias sociaux est de toujours présumer que rien n'est privé et que l'information à votre sujet sera utilisée de toutes sortes de façons sur lesquelles vous n'avez aucun contrôle. 
La deuxième règle est que vous avez le pouvoir et la responsabilité de vous assurer que les messages négatifs sur vous ne se rendent jamais sur Internet. Mieux encore, remplacez-les par des messages positifs que vous contrôlez. Pour vous donner une idée, cherchez votre nom sur Internet. Que trouvez-vous? Comment les autres interpréteront-ils tout statut, gazouillis, photo ou tout autre matériel qu'ils trouveront en ligne sur vous?

Troisièmement, rappelez-vous que l'érosion de la vie privée comporte ses avantages. De plus en plus, les discours publics nous forcent à sortir de l'isolement; les réseaux de communication encouragent la création de relations positives et de réseaux utiles qui compensent leur empiètement sur la vie privée. Comme nous le suggérons plus loin dans ce chapitre, vous pouvez tirer avantage des médias sociaux à l'école, au travail et dans la vie.

Quatrièmement, Internet est une autoroute express, ce qui veut dire qu'il est toujours bondé. Chaque minute équivaut à l'heure de pointe et les gens perdent leur sang-froid et se klaxonnent les uns les autres. Les médias sociaux profitent davantage aux rares personnes qui possèdent le sens critique et les aptitudes créatives pour passer au crible toutes les cochonneries qui s'y trouvent et faire ressortir ce qui n'en est pas. Si vous choisissez les chemins que vous empruntez et que vous gardez votre calme, la route sera beaucoup plus agréable et remplie d'aventures plaisantes.

Cinquièmement, si un problème survient sur les médias sociaux ou si vous faites une erreur, prenez les mesures nécessaires sur-lechamp. Supposons que vous avez envoyé un courriel, fait un commentaire ou affiché une photo et que vous le regrettez. Instinctivement, votre réaction sera d'attendre et d'espérer que personne ne le remarquera. N'attendez pas. Cela ne fera qu'aggraver le problème. Faites immédiatement des excuses à quiconque est susceptible d'être affecté. Assumez la responsabilité de la situation.

Vos excuses pourraient ressembler à ceci : "Chère Suzanne, je suis désolée du message déplacé que je t'ai envoyé il y a quelques minutes. Je l'ai écrit à la hâte et ne l'ai pas relu avant de l'envoyer. Sois assurée que je n'ai jamais voulu remettre en question ta compétence ou ta précieuse contribution au projet. J'ai mis tous les autres membres du groupe en copie de ce courriel pour qu'ils soient au courant. »

Les excuses n'ont pas besoin d'être longues, mais elles doivent être sincères et tomber à point nommé. Essayez également d'offrir vos excuses en personne. Ne les transformez pas en explications : «J'étais 
fatigué et débordé ce matin, et je devais également m'occuper d'une demande urgente. »

Si quelqu'un affiche des photos de vous en ligne, vous avez tout à fait le droit de demander qu'elles soient retirées. Si quelqu'un vous identifie dans une photo (tag), vous pouvez presque toujours retirer vous-même l'identification.

\section{Les médias sociaux et la création de relations}

Les carrières qui ont du succès ont toujours été dépendantes du réseautage et de la création de relations. Les médias sociaux peuvent servir à créer et à adapter vos réseaux. Ne pas en tirer profit, c'est tout simplement vous faire du tort. Comme nous l'avons indiqué dans le chapitre précédent, les médias sociaux facilitent la création d'un réseau de contacts et la planification d'entrevues d'information.

L'omniprésence des courriels, de Facebook et des autres services de messagerie est un bon exemple de la façon dont les relations se créent. Le courriel est parfois embêtant, mais il est de nos jours absolument essentiel aux affaires et à l'obtention de résultats. Aussitôt que vous envoyez un message, vous vous engagez envers une autre personne. Autrement dit, vous entrez dans une relation qui pourrait vous aider ou vous nuire quant à ce que vous voulez faire et où vous voulez aller. Faites attention chaque fois que vous envoyez un message, car chacun d'eux peut soit créer, soit briser une relation.

Par exemple, les étudiants communiquent habituellement par courriel avec leurs professeurs et les assistants à l'enseignement. Beaucoup d'étudiants sont tellement habitués à la satisfaction instantanée dans la vie de tous les jours qu'ils s'y attendent tout le temps, sans se poser de questions. C'est particulièrement vrai au début de la session: "Cher Professeur, est-ce que votre cours est facile ou difficile? », «Je ne trouve pas le livre du cours à la librairie. Que dois-je faire? », « Je veux changer de groupe de discussion », «J'ai été malade. Est-ce que j'ai raté quelque chose d'important? », «J'ai besoin de votre aide! », «Une réponse immédiate serait grandement appréciée ».

Ces messages ne sont souvent pas signés, n'indiquent pas de numéro étudiant ou de référence au cours dont il s'agit. Ils viennent parfois de comptes de courriel personnels. L'orthographe et la ponctuation y sont épouvantables, ce qui indique qu'ils ont probablement été rédigés rapidement, sans réflexion. C'est pourquoi ils finissent dans le dossier des messages supprimés. Dans leur quête de satisfaction 
immédiate, les étudiants oublient souvent que la communication n'est pas à sens unique.

Les étudiants qui exigent de leurs professeurs qu'ils leur répondent immédiatement réduisent à néant le potentiel de création de relations que peuvent avoir les courriels. Un courriel poli d'un étudiant qui s'identifie et identifie le cours en question crée une image positive dans l'esprit de celui qui le lit. Une grammaire et une ponctuation correctes, et de la concision, indiquent de la réflexion dans la composition du message et que l'étudiant a fait quelques démarches ( J'ai demandé aux employés de la librairie quand ils recevraient de nouveaux exemplaires, mais ils ne le savaient pas»), ce qui dénote de l'initiative. Un court message de remerciement pour l'information reçue consolide l'image positive ainsi créée.

Sur le marché du travail, seuls les idiots, les enragés ou les insouciants oseraient envoyer un courriel impoli. Ce courriel peut être ensuite envoyé à n'importe qui, n'importe où, n'importe quand et il demeure dans un dossier bien après qu'un commentaire oral aura été oublié.

Le désir de se protéger ou d'assurer ses arrières constitue une composante dominante et, selon nous, très toxique, de la communication électronique. Sauvegardez-vous les courriels que vous envoyez ? Est-ce pour prouver que vous n'avez pas fait d'erreur ou que d'autres en ont fait? L'objectif de cette accumulation de courriels est d'être au-dessus de tout blâme. Ceux qui cherchent d'abord et avant tout à se protéger sont soit de très mauvais employés, ou ils ont la malchance de travailler dans un environnement malsain qu'ils devraient penser à quitter.

Une autre composante de la communication électronique privilégie l'apparence au lieu de la substance. Les courriels sont souvent des demandes d'aide, une réalité qui a motivé certains employés à devenir des experts dans l'art d'avoir l'air d'aider. Après avoir donné une raison plus ou moins valable de ne pas être en mesure d'aider, ils finissent leur message par quelque formule du genre : « N'hésitez pas à communiquer avec moi si je peux faire quoi que ce soit pour vous aider. » Ceux qui évitent constamment de contribuer au succès des autres découvriront un jour que personne ne voudra les aider.

Vous devez évidemment vous protéger de ceux qui jettent le blâme injustement. Et refuser d'aider est parfois la bonne réponse, mais si vous utilisez ce genre de technique pour éviter de travailler avec les autres et de faire votre part pour le succès du groupe ou de l'organisation, vous n'irez pas loin. 
Beaucoup de scénarios malheureux commencent par une simple communication par courriel. Beaucoup de relations positives peuvent se développer si vous traitez le courriel comme une façon d'entrer en relation avec les autres.

Réfléchissez à la nature de vos échanges électroniques au travail ou à l'école. Sont-ils positifs, véhiculent-ils de l'optimisme, de l'organisation, du dynamisme, c'est-à-dire les qualités que tout le monde apprécie?

\section{Créer une image de marque}

Votre présence en ligne est remarquable et permanente parce que l'information n'y est jamais complètement effacée. C'est à vous de projeter et de protéger une image positive de vous en ligne. Cela commence par ne jamais mettre d'images ou faire de commentaires stupides ou salaces sur Internet.

Croyez-nous, un employeur ou un conjoint potentiel finira par découvrir ces manques de jugement et ne considérera pas sérieusement votre candidature. Vous pourrez peut-être parfois limiter les dommages, mais le plus sage est de ne simplement pas faire ce genre d'erreurs.

Comme on le dit dans l'univers des affaires, tout le monde est une marque, que ça lui plaise ou non. Vous avez même une image de marque si vous n'avez aucune présence en ligne! Elle est seulement négative et pourrait donner l'impression que vous n'avez rien accompli, que vous vivez en ermite ou que vous n'avez pas les compétences nécessaires pour utiliser les médias sociaux. L'image que vous projetez (ou non) dans le monde ne représente peut-être pas qui vous êtes vraiment ou complètement, mais elle est ce que le monde voit de vous. C'est pourquoi vous devriez vous appliquer à la développer et à la promouvoir comme toute image de marque.

Nous ne voulons pas dire par là que vous devez vous transformer en agent de marketing. Nous vous encourageons plutôt à créer une version virtuelle de vous-même qui vous aidera à atteindre vos objectifs à l'école, au travail et dans la vie.

Une manière simple d'y arriver est de créer votre propre site Web. Une page Web bien conçue, laquelle peut être créée facilement avec un gratuiciel de Weebly.com, vous aidera à contrôler les messages que vous voulez communiquer. Au prix de seulement quelques heures de travail, vous aurez un site que d'autres peuvent visiter, $y$ compris des employeurs potentiels. 
De plus, un site Web peut être l'emplacement principal de toute information à votre sujet, dont votre $\mathrm{CV}$. Vous pouvez y afficher vos dissertations (elles sont à vous, après tout) et toute autre réalisation : des photos, des œuvres d'art, une vidéo où on vous voit réciter un poème dans un cours d'anglais; n'importe quoi. Rappelez-vous toutefois que vous devez sélectionner soigneusement tout ce que vous affichez.

En faisant la promotion de vous-même, ne faites jamais l'erreur de penser que le grésillement est plus important que le steak, c'est-àdire que l'image a plus de valeur que le produit. Cela ne veut pas dire que vous devez vous retenir de montrer un peu de ce grésillement sur votre page Web. Faites preuve de créativité.

Quand vous donnez votre CV à un employeur potentiel, il est préférable de demeurer traditionnel parce que plusieurs recruteurs se méfient de tout ce qui est tape-à-l'œil. Dans le même ordre d'idées, les demandes de bourses ou d'admission aux études supérieures devraient être plutôt conservatrices. Cependant, une page Web vous permet une plus grande marge de manœuvre dans le développement de votre image, en plus de vous donner la chance de démontrer vos compétences en multimédia.

Même si votre page doit être propre, claire, nette, précise et professionnelle, vous pouvez y ajouter des hyperliens qui mèneront vos lecteurs vers des endroits un peu moins conventionnels s'ils choisissent de le faire. Utilisez-les pour présenter vos compétences et vos passions.

Le conseil le plus important pour la création de votre page Web est de conserver un ton optimiste, sans avoir l'air trop naïf. Éloignezvous de la négativité, même si vous croyez fermement qu'elle est intelligente, sophistiquée et discrète. Un corollaire de cela est que, si vous hyperliez à d'autres sites Web, assurez-vous que ceux-ci évitent aussi la négativité car on pourrait vous condamner par association.

Un site Web personnel est un outil extraordinairement puissant. Beaucoup de gens préfèrent la visite d'un site Web à la lecture d'un interminable CV ou d'une longue lettre d'accompagnement. Plusieurs personnes, y compris un conjoint potentiel, se tourneront vers votre site Web pour en apprendre davantage sur vous.

Il existe beaucoup d'applications qui vous permettent de créer et de manipuler du matériel multimédia d'une manière créative qui vous est propre. Dans l'univers d'Internet, où n'importe quel morceau de musique peut être jumelé à n'importe quelle photo, et où ce 
jumelage peut ensuite être manipulé pour pratiquement toutes les raisons possibles, l'investissement d'un peu d'énergie créative dans la création de votre site Web peut avoir des résultats extraordinaires.

Le professeur Klassen, qui n'est pas tout à fait aussi vieux que le professeur Dwyer, vient tout juste d'apprendre comment créer des sites Web. Son site personnel se trouve au www.thomasklassen. net. Quels choix a-t-il faits lors de la création de son site? Lequel des quatre groupes de compétences essentielles des professionnels (communication, résolution de problèmes, travail d'équipe et adaptation au changement) a-t-il mis en évidence ? En quoi ce site est-il différent de son site Web professionnel au klassen.blog.yorku.ca?

Quand vous créez votre image de marque, réfléchissez à la façon dont les autres pourraient vous interpréter. Trop de sites Web sont des exercices égoïstes en découverte et en promotion de soi (la forme la moins efficace de marketing).

Il est acceptable de montrer aux autres qui vous êtes et ce que vous aimez, mais la communication par Internet devrait toujours avoir pour objet le développement et l'entretien de relations. Cette suggestion se base sur la reconnaissance du fait qu'une page Web ou une présence en ligne est inévitablement publique. Même s'il n'est pas nécessaire d'éliminer tous les aspects privés et personnels (après tout, ils sont ce qui vous rend unique et intéressant), vous devriez exploiter à votre avantage la différence entre le public et le privé.

Si la création de votre propre page Web vous semble trop intimidante, joignez-vous à LinkedIn et créez-y un profil (voir www. linkedin.com). LinkedIn est un service de réseautage social, principalement utilisé par les professionnels. Il constitue une excellente façon de communiquer avec des gens qui partagent vos intérêts d'études ou de carrière, et de distribuer votre $\mathrm{CV}$. Nous recommandons à tout étudiant qui en est à sa dernière année d'études (voire avant) de se créer un profil LinkedIn.

Rappelez-vous toutefois que tout le monde peut voir votre profil LinkedIn. Assurez-vous qu'il reflète parfaitement votre CV et votre relevé de notes.

De plus en plus d'employeurs scrutent la présence d'employés potentiels en ligne afin de trouver des défauts et des failles, qu'ils exagèrent invariablement. Pourquoi ? Parce qu'un employé difficile, rebelle ou mal disposé représente un énorme problème pour une organisation. Ceux qui, imprudemment, ne perçoivent pas comment leur identité virtuelle peut influencer leur identité professionnelle ont 
un réveil brutal quand ils découvrent le manque de séparation entre les deux.

\section{La métaphore des sites Web de rencontre}

Ce livre s'appuie énormément sur la métaphore de la recherche d'un partenaire amoureux pour illustrer le besoin de choisir des cours qui vous plaisent puis, plus tard, un emploi qui vous correspond. Nous croyons en effet que la recherche du bon programme d'études et du bon travail ressemble beaucoup à la recherche de l'âme sœur, cette dernière impliquant le développement particulièrement sérieux de relations.

La plupart d'entre nous prennent très au sérieux la recherche d'un partenaire de vie; nous devrions faire de même dans notre recherche d'emploi postétudes.

L'utilisation de la métaphore de la recherche de l'âme sœur suppose deux choses. D'abord, que vous établissiez une connexion émotive entre la recherche de l'âme sœur et la recherche d'emploi, qui souligne des éléments importants et souvent négligés de cette dernière. Deuxièmement, que vous ayez déjà une meilleure compréhension de la recherche de l'âme sœur que de la recherche d'emploi.

Notre but premier n'est pas de faire la promotion des sites de rencontre, lesquels peuvent ou peuvent ne pas fonctionner selon les individus, mais plutôt de suggérer que, si vous prenez le développement de relations au sérieux, vous devez absolument prendre en considération l'environnement en ligne. De plus, nous citons en exemple le cas des rencontres sur Internet pour mettre en lumière ce dont on doit tirer avantage et ce qu'on doit éviter dans toute relation ayant une composante en ligne (il est de plus en plus difficile d'en imaginer une qui n'en ait pas).

Pour trouver quelqu'un à aimer, vous devez envoyer des signaux sur vous-même et sur votre disponibilité. Pour vos parents et vos grands-parents, cela s'est produit lors de rencontres en personne avec d'autres familles, au sein de groupes religieux et peut-être à l'école et au travail.

De nos jours, de plus en plus de personnes trouvent «l'amour de leur vie » en ligne ou sur des sites de rencontre qui exigent le partage d'informations détaillées. Tenter de faire des rencontres en utilisant des sites Web dans ce but signifie communiquer qui vous êtes, non seulement en faisant preuve d'intégrité, mais également avec 
intelligence critique et créativité. De plus, vous êtes responsable d'interpréter les signes et les signaux qu'envoient les autres. Une stratégie simple pour ce faire est de suivre l'exemple des employeurs : éliminez ceux qui n'entrent pas dans le moule ou qui envoient des messages qui évoquent instabilité, insécurité, manque de fiabilité ou tout autre trait de personnalité indésirable.

Quand vous êtes à la recherche d'un emploi, l'envoi de signaux est tout aussi important. Comme nous l'avons exposé dans le chapitre précédent, vous faites cela par réseautage, mais aussi, et de plus en plus, par votre présence en ligne, sur LinkedIn et sur votre propre site Web.

Les rencontres amoureuses sur Internet pourraient et devraient être une métaphore pour tout développement important de relations dans le monde d'aujourd'hui. Elles incarnent les défis auxquels font face les individus dans leur quête d'espace personnel et de relations profondes, dans un monde où les signes et les signaux abondent, mais sont souvent trompeurs. Penser que les aptitudes critiques et créatives ne sont utiles qu'à l'école ou au travail, c'est ignorer la très grande tâche de notre vie: trouver et donner de l'amour. Les aptitudes critiques et créatives demeureront peu gratifiantes si nous ne nous rendons pas compte à quel point elles ont aussi à voir avec les grands thèmes de la vie.

Les relations les plus importantes que nous avons, outre celles que nous avons avec nos parents, sont avec ceux que nous choisissons d'aimer. La relation amoureuse est à la fois le modèle idéal pour toute autre relation significative et l'environnement dans lequel nous nous développons. Trouver un environnement de travail tout aussi positif garantira pratiquement une vie épanouissante et heureuse. 


\title{
La réussite au travail et au-delà
}

\begin{abstract}
"Quelle est la recette du véritable accomplissement? À mon sens, elle est composée de seulement quatre ingrédients essentiels : choisissez une carrière que vous aimez, donnez-lui le meilleur de vous-même, sautez sur chaque occasion et soyez un bon joueur d'équipe. » - Benjamin F. Fairless
\end{abstract}

$\mathrm{V}$

ous avez décroché votre premier emploi après avoir obtenu votre diplôme. Félicitations! Pendant les premières semaines ou les premiers mois, vous vous sentirez peut-être comme quand vous avez commencé vos études postsecondaires. Vous vivrez une période de transition et de nouvelles attentes. Pratiquement tout vous semblera étranger et vous devrez tout comprendre rapidement. Heureusement, vos études vous auront préparé à l'absorption et à la compréhension d'un maximum d'informations et, avec un peu de chance, vous auront fourni les compétences pour que vous apportiez votre propre contribution.

Au début, il se peut que vous soyez un peu déçu par votre travail. Il avait semblé tellement passionnant pendant l'entrevue, mais maintenant que vous vous y habituez, vous vous rendez compte que vous avez vraiment commencé au bas de l'échelle et vous avez hérité de ce dont les autres ne voulaient pas : clients, étudiants, horaires de travail, projets et tâches. 
Ce n'est pas un hasard. Juste avant que vous arriviez dans le décor, vos collègues ont pris ce qu'il y avait de meilleur. Ne désespérez pas. Vous avez été embauché justement parce que personne d'autre ne pouvait ou ne voulait faire ces tâches plus ingrates et moins motivantes. Maintenant que vous faites partie de l'équipe, prouvez que vous pouvez apprendre rapidement et faire votre part, tout en acceptant de bonne grâce d'être le petit nouveau ou la petite nouvelle.

\section{Le premier emploi}

Les premiers collègues et les premiers supérieurs que vous aurez évalueront de près votre personnalité. Pendant ces moments difficiles, vous ferez bonne impression si vous vous appliquez à contribuer au lieu de vous plaindre, ce qui pourrait grandement vous rendre service quand des possibilités d'avancement se présenteront.

Les nouveaux employés souhaitent souvent progresser rapidement. Détrompez-vous si vous croyez qu'après deux semaines ou deux mois en poste, vous aurez découvert une façon révolutionnaire de faire les choses et à laquelle personne n'a jamais pensé auparavant. Il est très peu probable que vous résoudrez un problème de longue date en aussi peu de temps.

Croire que vous êtes meilleur que les autres risque également d'insulter et d'irriter vos collègues et vos supérieurs. Vous serez perçu comme quelqu'un d'arrogant qui n'est pas un joueur d'équipe. Il est important d'avancer prudemment à ce stade, si ce n'est que pour faire preuve de courtoisie et de respect.

Ne rejetez pas non plus vos observations du revers de la main. Le fait que vous soyez nouveau et que vous ayez des connaissances et une expertise fraîchement sorties des études peut également jouer à votre avantage. Vous posez en effet un regard neuf sur les habitudes et les processus, ce qui vous aidera à apporter votre contribution.

Tenez un journal ou prenez des notes pendant les quelques premières semaines, car, dès que vous aurez fait partie d'une organisation pendant un certain temps, vous aurez tendance à accepter les choses comme elles sont. Tout le monde a une inclination naturelle à trouver la routine réconfortante et à devenir, par conséquent, moins apte à remettre en question les idées tenues pour acquises. Vous aurez peutêtre même davantage cette tendance parce que vous voudrez désespérément vous sentir à l'aise dans votre nouveau poste. 
Pour parvenir à contribuer positivement au travail, en particulier par votre capacité à penser de manière critique, ou originale, il est essentiel que vous essayiez de préserver le plus longtemps possible votre regard neuf, votre curiosité et votre aptitude à remettre les idées en question. Tout cela combiné à un peu plus d'expérience, vous ajouterez de la valeur à n'importe quelle organisation.

Commencez également à vous spécialiser au travail. Devenez plus efficace que d'autres dans l'exécution de certaines tâches. Il s'agira peut-être des corvées que tout le monde fuit. Cependant, maîtriser certaines d'entre elles vous permettra d'ajouter immédiatement de la valeur, vous donnera la chance de vraiment comprendre le travail ou le processus, et indiquera le genre de personne et d'employé que vous deviendrez.

Les recruteurs d'entreprise se plaignent souvent que beaucoup de diplômés postsecondaires manquent d'aptitudes collaboratives. Vous ferez donc bonne impression si vous faites preuve d'esprit d'équipe. C'est à ce moment que vous récolterez les fruits de votre expérience des travaux de groupe faits pendant vos études.

Si vous avez naturellement un bon esprit d'équipe, vous voudrez peut-être immédiatement faire partie des projets. Vous risquez ici d'avoir l'air trop arrogant et de vouloir vous mettre trop de l'avant. Soyez patient. Vos supérieurs et vos collègues voudront sans doute vous observer et évaluer votre talent avant de vous inclure. Ne vous en faites pas. Ils constateront votre potentiel bien assez tôt! Quand cela se produira, vous serez peut-être inondé de demandes d'aide.

Les organisations complexes d'envergure divisent généralement le travail en projets et les employés participent habituellement à plusieurs d'entre eux en même temps. Conséquemment, ils jouent plusieurs rôles différents. L'expérience acquise en groupes lors de vos études postsecondaires vous sera ici très utile.

Il est aussi possible que vous ayez plusieurs différents superviseurs et collègues, selon le nombre de projets auxquels vous participerez. Ils n'auront peut-être pas conscience que vous travaillez sur plusieurs projets à la fois. Même s'ils en sont conscients, la nature humaine étant ce qu'elle est, ils croiront que leur projet est le plus important et que leur échéancier et leur date limite sont les seuls qui comptent!

Heureusement, vous aurez beaucoup d'expériences semblables avec vos professeurs, qui vous demandent tous de rendre vos devoirs la même semaine. Vous aurez appris à gérer votre temps et les 
échéances. Idéalement, vous aurez aussi appris que certaines tâches sont plus difficiles que d'autres et que certains emplois et certains professeurs/employeurs sont plus exigeants que d'autres.

Donnez-vous le temps de vous ajuster. Visez la compétence, voire l'excellence, mais ne vous attendez pas à la perfection. Personne n'est parfait, mais plusieurs se sont épuisés à essayer de l'être!

Pendant que vous apprenez à faire votre travail, servez-vous des compétences que vous avez développées pendant vos études postsecondaires. Faites-vous un horaire, comme vous l'avez fait à l'école. Organisez vos tâches en ordre de priorité et ajoutez toujours un petit quelque chose de plus à ce que vous faites, par de la recherche ou par votre façon de terminer les projets. Utilisez vos aptitudes critiques pour déterminer ce qui est essentiel. Par-dessus tout, faites preuve d'initiative et acceptez les critiques avec grâce. Une bonne partie de votre succès au travail dépendra de ce que vous avez ou non profité de vos études pour développer et perfectionner vos aptitudes.

Au lieu d'être une période d'adaptation stressante, vos premières semaines ou vos premiers mois sur le marché du travail pourraient plutôt être amusants et stimulants. Et puis, vous gagnez de l'argent! Contrairement à l'époque où vous étiez aux études, vous pourriez même être libre la fin de semaine ! Les travaux de session et les dissertations sont choses du passé. En plus, vous rencontrez de nouvelles personnes et apprenez de nouvelles aptitudes et habiletés.

Il arrive occasionnellement que l'enthousiasme persiste pendant toute une carrière. Généralement, par contre, celui-ci finit par se dissiper. Après quelques mois ou un an au travail, beaucoup d'employés se sentent un peu démotivés. Ils sont au même bureau, dans le même cubicule, et font les mêmes tâches que lorsqu'ils sont arrivés en poste. Ils ne s'épanouissent pas au travail et doutent que ce qu'ils font soit même reconnu. La routine quotidienne s'installe, avec comme seul objectif, très loin à l'horizon, deux petites semaines de vacances, au lieu de trois ou quatre mois comme lorsqu'ils étaient aux études. L'enthousiasme du début commence à disparaître et ils feraient tout pour un changement. N'importe quel changement!

Ne vous en faites pas, c'est normal. Contrairement à vos professeurs, les gestionnaires sont généralement avares de notes ou de commentaires. Ils ne produisent pas de relevés de notes, résumant ce que vous avez appris ou réalisé (les évaluations de rendement n'ont généralement lieu qu'une fois l'an). Au lieu de travailler avec plusieurs professeurs et beaucoup d'autres étudiants, vous avez un 
groupe social beaucoup plus restreint pour vous stimuler.

Beaucoup d'entreprises et de grandes organisations ont une structure hiérarchique. Vous aurez peut-être l'impression que vous êtes au bas d'une interminable échelle, que tout le monde est au-dessus de vous et que votre comportement est continuellement en observation. Bien sûr, pendant vos études, vos professeurs avaient une certaine influence sur vous, mais elle se limitait à un seul cours que vous pouviez toujours décider d'abandonner. Et pour chaque mauvais professeur ou assistant à l'enseignement, il y en avait probablement un excellent qui vous forçait à vous dépasser ou qui vous motivait.

Ironiquement, après trois ou quatre années à attendre l'obtention de votre diplôme et à rêver d'en avoir terminé une fois pour toutes avec les études, vous vous prendrez peut-être à vous ennuyer de votre vie d'étudiant. Au moins, les études vous offraient quelque chose de nouveau au début de chaque session: de nouveaux cours, de nouveaux camarades de classe, de nouveaux professeurs et de nouveaux livres.

Si ça vous arrive, rappelez-vous que les choses ne sont pas aussi terribles qu'elles en ont l'air. Vous habituer aux rythmes du monde du travail pourrait vous prendre un an, voire deux, mais vous vous rendrez graduellement compte que les récompenses que procure un travail exigeant, mais épanouissant, compensent la perte de votre vie d'étudiant. Il est très satisfaisant de maîtriser des tâches du monde réel. De plus, vous pouvez toujours utiliser vos aptitudes critiques et créatives pour rendre vos projets plus gratifiants.

\section{Se garder à jour}

Si vous vous ennuyez des études, vous pouvez toujours suivre un cours. S'il est en lien avec votre travail, beaucoup d'employeurs iront même jusqu'à payer une partie des frais. Cependant, pourquoi ne pas choisir un sujet qui n'a rien à voir avec le travail, quelque chose d'autre qui vous intéresse? C'est là une bonne façon de rester créatif.

Pour déterminer ce qui vous manque exactement, faites appel à vos aptitudes en résolution de problèmes, telles que décrites au chapitre 7. Si le changement vous manque, essayez de trouver des façons de varier votre travail. Si c'est de développement personnel dont vous avez besoin, profitez de vos soirées et de vos fins de semaine pour vous découvrir d'autres intérêts. Si vous voulez approfondir vos compétences ou varier vos perspectives, essayez de trouver comment 
vous pourriez y arriver au travail. Vous êtes devenu un expert de l'apprentissage ; commencez à vous motiver et à apprendre au travail et à l'extérieur de celui-ci.

Par-dessus tout, ne vous limitez pas. Une formation générale, en particulier en sciences humaines, vous offre quelque chose qu'une formation spécialisée ou professionnelle ne peuvent livrer : une grande capacité d'adaptation. Tout employeur est à la recherche d'employés qui savent s'adapter, mais vous pouvez également en tirer avantage.

En effet, l'avantage principal de votre éducation postsecondaire, par rapport au marché de l'emploi, est la flexibilité. Elle vous donne la capacité incomparable de faire ce qui vous intéresse : travail autonome, bénévolat, travail à temps plein et à temps partiel, contrats, permanence, travail à l'étranger, etc.

Les étudiants se demandent souvent: "À quoi ça sert, un diplôme d'études postsecondaires? » et il est difficile de répondre à cette question. Votre diplôme sert à tellement de choses! Les diplômés se retrouvent dans toute sorte de postes qui semblent n'avoir aucun lien avec leur domaine d'études.

Il est tout aussi fascinant de constater à quel point ces diplômés réussissent bien dans autant de domaines. Leurs compétences les rendent indispensables aux yeux de leurs employeurs et leur permettent de gravir les échelons raisonnablement rapidement, mais ils ont également la liberté inégalée de changer d'emploi et même de se lancer en affaires.

Par contre, avec la liberté et la flexibilité vient la responsabilité de découvrir leurs intérêts et de les développer.

\section{Faire la transition}

La transition de l'école au travail ne se fait pas toujours en douceur. Même s'il peut être stressant, l'environnement scolaire devient parfois très réconfortant pour les étudiants. Au moment d'obtenir leur diplôme, ils auront compris le fonctionnement de l'école et s'ils sont de bons étudiants, il se peut qu'ils aient aussi eu beaucoup de plaisir.

Pendant les études postsecondaires, votre travail commence et se termine en quelques mois seulement, du début de la session à la période des examens. Votre progression est régulière et vous obtenez un diplôme en un court laps de temps. Au travail, votre cheminement de carrière devient beaucoup plus élastique et les changements 
significatifs prennent beaucoup plus de temps à se produire que vous ne le croyiez.

On dit que le changement caractérise le monde du travail, mais cela ne s'appliquera pas nécessairement à votre propre avancement professionnel. Ne soyez pas trop impatient. Vous finirez bien par trouver votre zone de confort au travail. Les baby-boomers ont commencé à prendre leur retraite et vous aurez considérablement plus de chances d'avancement dans quelques années. Si vous venez tout juste d'entamer vos études postsecondaires, vos perspectives d'avenir sont particulièrement bonnes.

Si vous êtes malheureux ou impatient au travail, votre emploi ne vous convient peut-être pas et vous devriez peut-être penser à changer. N'envisagez surtout pas ce changement comme quelque chose dont vous devriez avoir peur. Voyez-le plutôt comme une occasion de croissance et une possibilité de retrouver votre enthousiasme.

Après quelques mois ou une année, vous pourriez trouver que votre emploi n'est pas aussi gratifiant que vous le croyiez. C'est tout à fait normal. Après tout, il y a très peu de chances que votre premier emploi corresponde à votre emploi idéal. La plupart des gens ont deux emplois pendant les deux années qui suivent l'obtention de leur diplôme. Les stratégies et les suggestions incluses dans ce livre peuvent vous aider pendant que vous êtes à la recherche de la correspondance parfaite entre vous et les exigences d'un poste.

Notre conseil au sujet de la façon de choisir vos cours s'applique également très bien au choix d'un emploi. Suivez votre instinct. Consultez votre réseau et réfléchissez à vos intérêts, à vos désirs et à vos rêves. Après une année ou deux sur le marché du travail, vous serez différent de la personne que vous étiez aux études. Nous exposons ci-dessous les stratégies que tout professionnel doit adopter, peu importe à quel stade de sa carrière il se trouve.

\section{Passer à autre chose}

Dès que vous commencez à travailler à temps plein, votre $\mathrm{CV}$ devient plus important que jamais. Mettez-le à jour toutes les deux semaines ou chaque mois, au fur et à mesure que vous entreprenez de nouvelles tâches et que vous développez de nouvelles habiletés. Si vous poursuivez des études à temps partiel, assurez-vous de l'indiquer. Vous seriez étonné de la fréquence à laquelle on peut vous demander votre $\mathrm{CV}$, parfois à la dernière minute. 
Gardez également à jour votre liste de références. Votre employeur sera toujours votre référence clé. Ne coupez donc jamais les ponts au travail, même si cela signifie parfois vous mordre la langue et ravaler votre orgueil, aussi difficile que cela puisse être. Dans plusieurs années, vous serez content de ne pas avoir dit votre façon de penser et vous vous souviendrez peut-être avec beaucoup d'affection de ces employeurs difficiles. De la même façon, vous verrez peut-être autrement votre professeur exigeant!

Dès que vous avez décidé de passer à autre chose, vous devez faire des recherches. Vous aurez élargi votre réseau pendant que vous travaillez et pouvez ainsi commencer à communiquer avec vos contacts clés. Il est préférable de chercher un emploi alors que vous en avez un; ne donnez donc pas votre démission avant d'avoir reçu une offre ferme.

Évidemment, votre disponibilité pour chercher dépend de votre emploi. Certains emplois sont tellement exigeants et prennent tellement de votre temps que vous n'aurez tout bonnement pas le loisir de réfléchir, encore moins de vous mettre à la recherche d'un emploi. De plus, certains emplois ne permettent pas de construire un bon réseau de contacts et finissent par être des impasses de carrière. Serrez alors les dents : remettez votre démission et commencez à chercher un nouvel emploi.

Si vous n'avez pas déjà un nouveau poste quand vous quittez votre emploi, attendez-vous à ce que plusieurs mois s'écoulent avant que vous n'en trouviez un. Surtout, ne perdez pas confiance en vous pendant cette période. Quand vous doutez de vous, pensez à ce que vous avez réussi à accomplir en obtenant un diplôme d'études postsecondaires. Si vous avez pu faire ça, vous pouvez faire n'importe quoi !

Essayez d'éviter de vous apitoyer sur votre sort. Vous ne vous sentirez pas en chômage si vous considérez votre recherche d'emploi comme un travail à temps plein. Levez-vous tôt chaque matin, habillez-vous comme si vous alliez au travail, développez votre réseau de contacts, faites des entrevues d'information, repensez votre cheminement de carrière et évaluez vos options. La recherche d'emploi est l'emploi le plus difficile que vous aurez. Si vous la faites bien, vous n'aurez pas le temps de déprimer et vous finirez par réussir.

Une des clés du succès, que nous n'avons que trop brièvement mentionnée dans ce livre, est la confiance. Le succès à l'école donne une forme de confiance; le succès au travail en insuffle une autre. 
Le fait d'être en chômage et à la recherche d'un emploi mine généralement la confiance en soi et c'est pourquoi nous recommandons de chercher un nouvel emploi alors que vous en avez toujours un. Même si vous vous retrouvez sans emploi, vous pouvez toujours trouver réconfort et confiance dans le fait que vous avez réussi à surmonter les obstacles et à obtenir un diplôme d'études postsecondaires.

Vous pouvez également utiliser vos habiletés en recherche et en lecture pour affronter les périodes plus sombres de votre vie et développer les aptitudes personnelles qui amélioreront vos chances de réussir. Bien que l'anxiété et la dépression sévissent partout, il existe énormément de documentation sur la façon d'améliorer son bonheur et sa confiance.

\section{On n'arrête jamais d'apprendre}

Vous n'arrêterez pas d'apprendre quand vous commencerez à travailler. Par exemple, beaucoup de ceux qui ont actuellement des postes de cadres utilisaient des bouliers quand ils étaient au secondaire. Ils n'ont connu les ordinateurs que très tard dans leur carrière. Pensez à tout l'apprentissage que ça a dû nécessiter! Vous ferez face au même genre de situation au fur et à mesure que la technologie, les lois et les normes professionnelles et technologiques évolueront au cours de votre carrière.

La fluidité et la flexibilité vont de pair avec le marché global. Dans un monde qui est en changement constant, les compétences et le savoir-faire que vous avez développés pendant vos études sont plus pertinents que jamais, mais il n'en tient qu'à vous, pas à vos parents, à vos professeurs ou à vos employeurs, de les actualiser et de les appliquer au présent.

\section{Les études supérieures, les formations professionnelles et techniques, et les cours en ligne}

Après environ une année sur le marché du travail, vous aurez probablement une meilleure idée de ce qu'est votre emploi idéal. Vous déciderez peut-être à ce moment-là de retourner aux études pour compléter votre formation. Les écoles de formation professionnelle et les programmes d'études supérieures voient habituellement d'un bon œil les candidats qui ont choisi de travailler à temps plein après avoir obtenu un premier diplôme. Pour certains programmes de maîtrise en 
administration des affaires (MBA), travailler à temps plein pendant plusieurs années est en fait une exigence d'admission. Assurez-vous d'utiliser votre expérience de travail à votre avantage.

Les programmes d'études supérieures et les écoles professionnelles veulent avant tout admettre des individus qui termineront leur programme, pour ensuite contribuer à la profession. Faire une demande d'admission à ces écoles est un peu comme postuler un emploi. Comme les employeurs, celles-ci cherchent à éviter les étudiants ayant des problèmes parce qu'ils ne sont pas à leur place ou qu'ils ne peuvent pas suivre les règles.

Les programmes d'études supérieures et les écoles professionnelles ne s'intéressent pas d'emblée à l'intelligence ou au potentiel scolaire des candidats. Ils veulent par-dessus tout savoir si les candidats ont ce qu'il faut pour terminer le programme et devenir de bons collègues au sein de la profession.

Dans votre demande d'admission, faites la preuve que vous avez sérieusement évalué la manière dont vous pouvez (ou voulez) contribuer à votre profession de choix. Utilisez des références de votre réseau de contacts pour appuyer vos affirmations. Faites preuve de ténacité si vous ne réussissez pas du premier coup ou essayez de trouver d'autres façons d'atteindre votre but, comme des études à temps partiel ou des études en ligne. Il y a plusieurs façons de se rendre à destination.

Si vos notes laissent à désirer, expliquez pourquoi. Fournissez ensuite la preuve que votre capacité à apprendre et votre motivation se sont améliorées depuis l'obtention de votre diplôme. Obtenir quelques bonnes notes dans des cours à temps partiel pourrait ici vous servir.

Vos relevés de notes ne refléteront pas avec exactitude l'ensemble de vos compétences et de vos habiletés. Le bénévolat pourrait ici aider à combler les trous. Certaines professions, comme en éducation et en gestion, donnent presque autant de valeur aux activités de bénévolat qu'aux résultats scolaires.

Les candidats à certains programmes d'études supérieures ou professionnels doivent passer des examens normalisés comme le LSAT, GMAT ou le TAGE MAGE, précédemment mentionnés au chapitre 3. La meilleure façon de réussir ces tests est de vous familiariser avec les questions qu'on y pose. Il existe des milliers de sites Web qui fournissent des questions pour vous exercer. Vous pouvez les trouver en entrant des termes comme «échantillons de questions GMAT/ LSAT/TAGE MAGE ». Il existe également plusieurs livres dédiés à la préparation à ces examens. Référez-vous aussi à la section sur les tests 
à choix multiples au chapitre 3 de ce livre. Ceux qui s'exercent à ce genre de tests obtiennent des résultats beaucoup plus élevés que ceux qui ne le font pas.

Vous devrez soumettre des lettres de recommandation avec votre demande d'admission à une école professionnelle ou à un programme d'études supérieures. Comme certaines de ces lettres proviendront de vos professeurs, vous auriez intérêt à garder contact avec quelquesuns d'entre eux.

Suivez les étapes suivantes pour demander une lettre de recommandation pour un dossier d'admission à un programme d'études supérieures (ou pour une demande d'emploi):

- Donnez des détails sur l'endroit où vous demandez l'admission et pourquoi vous l'avez choisi ;

- Demandez à la personne si elle peut rédiger «une lettre de recommandation solide». Si la réponse est: «Je ne suis pas certaine» ou "Laisse-moi y penser», dites-lui poliment que vous demanderez à quelqu'un d'autre. Quand vous demandez une lettre de recommandation, vous devez vous assurer que la personne fera de vous le meilleur portrait possible. Pour différentes raisons, il se peut que certaines personnes ne soient pas capables de le faire. Si cela arrive, trouvez quelqu'un d'autre ;

- Envoyez une copie de votre relevé de notes ou une liste de vos cours et de vos notes à ceux qui rédigeront une lettre de recommandation. Pour vos professeurs, vous pouvez également faire la liste des travaux que vous avez faits ;

- Incluez votre CV. Plus vous donnez d'informations à votre sujet, plus la lettre de recommandation a de chances de vous être utile. Si vos notes se sont récemment améliorées ou si vous avez accompli d'autres réalisations pertinentes, soulignez-le. Faites ressortir toute réalisation en lien avec vos études ou toute autre activité ;

- Ajoutez une ébauche de votre lettre d'intention. Elle aidera votre professeur à comprendre vos objectifs à long terme et les raisons pour lesquelles vous soumettez une demande d'admission au programme en question ;

- Expliquez comment soumettre la lettre ou la référence (électroniquement ou sur papier) et assurez-vous d'indiquer la date limite.

$\mathrm{Si}$ vous suivez les étapes ci-dessus et que vous vous y prenez au moins quatre semaines d'avance, vous aurez la meilleure lettre de 
recommandation possible, qui fera même probablement mention de vos grandes aptitudes organisationnelles. Une demande de dernière minute se soldera par une lettre écrite à la va-vite, à laquelle il manque de l'information, et vous aurez peu de chances d'être accepté dans le programme. Faites un suivi de rappel à vos références une semaine avant la date limite.

La composante clé d'une demande d'admission à un programme d'études supérieures est la lettre d'intention. Elle ressemble beaucoup à la lettre d'accompagnement d'une demande d'emploi. Vous devez y être tourné vers l'avenir : ceux qui la liront veulent savoir ce que vous accomplirez dans le futur comme diplômé et comme professionnel. Ils ne sont pas vraiment intéressés au passé.

La lettre d'intention n'est pas une autobiographie ou une justification des résultats que vous avez obtenus dans le passé. Il s'agit plutôt d'un résumé de ce que vous avez l'intention d'accomplir en tant qu'étudiant aux études supérieures : quel sujet ou quelle question vous souhaitez explorer et comment vous comptez le faire. Vous pouvez également mentionner ce qui vous a incité à prendre la décision de faire une demande d'admission au programme: un livre précis, un auteur ou une théorie en particulier. La lettre d'intention est une dissertation. Abordez-la comme telle.

Les demandes d'admission aux études supérieures ou aux écoles professionnelles exigent un travail considérable et requièrent beaucoup de recherches. La date limite de soumission de la demande est souvent une année avant le début du programme. Vous devez avoir une idée précise de la recherche que vous voulez faire ou du programme que vous souhaitez intégrer. Une demande faite rapidement, comme un $\mathrm{CV}$ peu soigné, constitue une perte de temps absolue. Faites-nous confiance: nous avons beaucoup d'expérience dans ce domaine.

\section{Les collèges communautaires et la formation en ligne}

Il arrive parfois qu'un diplôme collégial ou un certificat viennent compléter une formation universitaire (ou vice versa) et vous permettent de mieux réussir sur le marché du travail. La combinaison de cours universitaires et de cours collégiaux est d'ailleurs beaucoup plus fréquente qu'auparavant.

Encore une fois, vous devez faire des recherches à ce sujet et consulter votre réseau pour déterminer s'il s'agit là de la meilleure 
option pour vous. Généralement, un diplôme d'études collégiales après des études universitaires est une bonne solution si vous n'avez pas beaucoup d'expérience de travail ou si vous savez exactement quel genre d'emploi vous voulez.

En raison de leur disponibilité, les cours et les programmes en ligne, lesquels peuvent parfois être suivis à temps partiel, offrent une autre avenue pour quiconque souhaite faire davantage d'études. Au travail, vous devrez souvent acquérir une compétence ou un savoirfaire que vous ne possédez pas. Un programme en ligne pourrait être exactement ce dont vous avez besoin dans ce cas.

Allant de la négociation à la rédaction de chansons, en passant par l'histoire du monde enseignée par des experts provenant des quatre coins de la planète, les cours en ligne sont d'une variété impressionnante. Certains sont même gratuits et disponibles quand et où ça vous convient ${ }^{1}$.

La plupart des étudiants trouvent que les cours en ligne sont ardus parce qu'ils exigent d'être très motivés. Par contre, ceux qui les font pour les bonnes raisons et qui utilisent les compétences en écoute, en lecture et en écriture décrites dans ce livre les réussissent habituellement très bien.

\section{Cumuler les succès}

La route qui mène à l'emploi idéal n'est finalement que ça : une route. Vous atteindrez peut-être votre emploi idéal pour découvrir qu'il ne l'est pas tant que ça, finalement. La vie est parfois ainsi faite. Nous devrions prendre les paroles de ces vieux croulants de rockers que sont les Rolling Stones: "You can't always get what you want. But if you try sometimes, you just might find, you get what you need. " «Tu ne peux pas toujours avoir ce que tu veux. Mais si tu essaies parfois, tu pourrais trouver ce dont tu as besoin »).

Vous rencontrerez des difficultés en cours de route, peut-être à cause de votre sexe, de votre origine ethnique ou de votre personnalité. Encore une fois, la clé est de vous faire confiance et d'essayer de réaliser vos rêves. Si vous ne vous sentez pas bien au travail, c'est probablement parce que votre expertise et vos intérêts ne correspondent pas aux exigences du poste.

Plus vous progresserez dans le monde du travail, plus vous gagnerez en expérience (en particulier celle qui vous convaincra que vous devez suivre votre instinct!) et trouverez des collègues et des 
associés fiables qui vous conseilleront. L'appui de votre réseau sera essentiel à votre succès et à votre bonheur. Un ou plusieurs de vos professeurs pourraient bien en faire partie, tout comme certains de vos camarades d'études actuels.

Peu importe où vous irez, les connaissances et les compétences acquises au cours de vos études vous seront toujours utiles : communication, résolution de problèmes, travail d'équipe et adaptabilité. $\mathrm{Si}$ vous en faites bon usage, vous êtes assuré de finir par trouver le succès.

\section{Note}

1. Pour commencer, consultez le «Catalogue» de Coursera à www.coursera.org/ ou la liste des cours à www.coursera.org/courses?languages=fr, ou encore Class Central au www.class-central.com/(en anglais seulement). Vous trouverez également de l'information sur le site Web de l'Université TELUQ au www.teluq.ca, ainsi que sur ceux des diverses universités canadiennes. 


\section{Autres ressources}

\section{Livres}

Babcock, L., et S. Laschever. Ask for It: How Women Can Use the Power of Negotiation to Get What They Really Want. New York, Random House, 2009.

Bailey, S. Academic Writing for International Students of Business, New York, Routledge, 2011.

BAIn, K. What the Best College Students Do, Cambridge, MA, Harvard University Press, 2012.

Bolles, R.N. What Color Is Your Parachute? 2015: A Practical Manual for JobHunters and Career-Changers, Berkeley, Ten Speed Press, 2014.

Bolles, R.N. De quelle couleur est votre parachute? Un guide pratique pour les chercheurs d'emploi et les personnes en transition de carrière, Repentigny, QC, Les Éditions Reynald Goulet, 2014.

Bowell, T., et G. Kemp. Critical Thinking : A Concise Guide, New York, Routledge, 2010.

Bureau de la traduction. Le guide du rédacteur, $2^{\mathrm{e}}$ éd., Ottawa, ON, Travaux publics et Services gouvernementaux Canada, 1996.

Burke, D., et J. Pieterick. Giving Students Effective Written Feedback, Maidenhead, UK, McGraw-Hill Education, 2010.

Chingouris, L. The Secret to Getting a Job after College: Marketing Tactics to Turn Degrees into Dollars, New York, Brand New World, 2011.

Cioffi, F.L. The Imaginative Argument : A Practical Manifesto for Writers, Princeton, Princeton University Press, 2005.

Congru, C. La ponctuation correcte, Paris, France, De Vecchi, 2005. 
Cottrell, S. Critical Thinking Skills : Developing Effective Analysis and Argument, New York, Palgrave Macmillan, 2011.

De VILlERs, M.-É. La nouvelle grammaire en tableaux, 6e éd., Montréal, QC, Québec Amérique, 2015.

De Villers, M.-É. Multidictionnaire de la langue française, $6^{\mathrm{e}}$ éd., Montréal, QC, Québec Amérique, 2015.

Dionne, B. Pour réussir: Guide méthodologique pour les études et la recherche, $6^{e}$ éd., Montréal, QC, Chenelière Éducation, 2013.

FARID, G. Mieux comprendre le participe passé, Montréal, QC, Éditions Nouvelles, 2004.

FitzPAtrick, M. Engaging Writing 2 : Essential Skills for Academic Writing, New York, Pearson Education, 2011.

Forest, C., et D. Boudreau. Le Colpron: le dictionnaire des anglicismes, 4e éd., Montréal, QC, Beauchemin, 1998.

Gilbert, M. How to Win an Argument, New York, John Wiley, 1996.

Gill, C.M. Essential Writing Skills for College and Beyond, Blue Ash, OH, Writer's Digest Books, 2014.

Gillett, A., A. Hammond, et M. Martala. Inside Track to Successful Academic Writing, Harlow, UK, Pearson Education, 2009.

Harvard University Business School. Giving Presentations : Expert Solutions to Everyday Challenges, Boston, MA, Harvard Business School Press, 2007.

Hewings, M. Advanced Grammar in Use, Cambridge, Cambridge University Press, 2005.

Jimenez, A., et J.-E. Tadlaoui. Guide méthodologique universitaire : un programme en 12 semaines, Montréal, QC, Presses de l'Université de Montréal, 2011.

Laurencelle, L. Abrégé sur les méthodes de recherche et la recherche expérimentale, Sainte-Foy, QC, Presses de l'Université du Québec, 2005.

Lispon, C. Doing Honest Work in College: How to Prepare Citations, Avoid Plagiarism, and Achieve Real Academic Success, Chicago, University of Chicago Press, 2004.

Malo, M. Guide de la communication écrite au cégep, à l'université et en entreprise, Montréal, QC, Québec Amérique, 1996.

McMillan, K., et J. Weyers. How to Write Essays and Assignments, Harlow, UK, Pearson Education, 2010.

Messenger, W., et al. The Canadian Writer's Handbook, Don Mills, ON, Oxford University Press, 2014.

Murphy, R. English Grammar in Use, Cambridge, Cambridge University Press, 2004.

Newport, C. How to Become a Straight-A Student: The Unconventional Strategies Real College Students Use to Score High While Studying Less, New York, Broadway, 2007.

Northey, M., et J. McKibbin. Making Sense: A Student's Guide to Research and Writing, Don Mills, ON, Oxford University Press, 2012. 
Northey, M., L. Tepperman, et P. albanese. Making Sense in the Social Sciences : A Student's Guide to Research and Writing, Don Mills, ON, Oxford University Press, 2012.

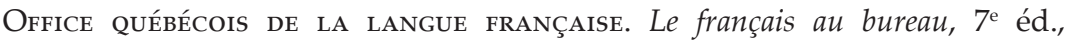
Montréal, QC, Publications du Québec, 2014.

Oshima, A., et A. Hogue. Writing Academic English: Level 4, White Plains, NY, Pearson/Longman, 2006.

Poirier, M. La grammaire expliquée, $4^{\mathrm{e}}$ éd., Montréal, QC, Isabelle Quentin éditeur, 2014.

Pollak, L. Getting from College to Career: Your Essential Guide to Succeeding in the Real World, New York, Harper, 2012.

Powers, P. Winning Job Interviews, Franklin Lakes, NJ, Career Press, 2005.

Provost, M.A., et al. Normes de présentation d'un travail de recherche, 4e éd., Trois-Rivières, QC, Éditions SMG, 2010.

Ramat, A., et A.-M. Benoit. Le Ramat de la typographie, 10éd., Montréal, QC, 2012.

Reinders, H., N. Moore, et M. Lewis. The International Student Handbook, London, Palgrave Macmillan, 2008.

Reynolds, G. Presentation Zen: Simple Ideas on Presentation Design and Delivery, Berkeley, New Riders, 2012.

Ruggerio, V.R. Becoming a Critical Thinker, Boston, MA, Houghton-Mifflin, 1996.

Runion, M. How to Use Power Phrases to Say What You Mean, Mean What You Say, and Get What You Want, New York, McGraw-Hill, 2004.

Schultze, Q.J. Resume 101 : A Student and Recent-Grad Guide to Crafting Resumes and Cover Letters That Land Jobs, New York, Ten Speed Press, 2012.

Shea, V., et W. Whitla. Foundations: Critical Thinking, Reading and Writing, Toronto, Pearson, 2001.

Simard, J.-P. Guide du savoir-écrire, nouvelle édition revue et corrigée, Montréal, QC, Éditions de l'Homme, 2005.

Swales, J.M., et C.B. Feak. Academic Writing for Graduate Students, Ann Arbor, University of Michigan Press, 2007.

Swan, M. Practical English Usage, Oxford, Oxford University Press, 2005.

Tanguay, B. L'art de ponctuer, 3e éd., Montréal, QC, Québec Amérique, 2006.

Tissington, P., M. Hasel, et J. Matthiesen. How to Write Successful Business and Management Essays, Los Angeles, Sage, 2009.

Van Roey, J., S. Granger, et H. Swallow. Dictionnaire des faux amis, $3^{e}$ éd., Bruxelles, Belgique, Duculot, 1998.

Westerfield, J. I Have to Give a Presentation, Now What?! New York, Silver Lining Books, 2002.

YAte, M. Resumes That Knock'em Dead, Avon, MA, Adams Media, 2004. 


\section{Sites Web}

Gouvernement du Canada, [s.d.], Ressources du Portail linguistique du Canada. Tiré de www.noslangues-ourlanguages.gc.ca

Office québécois de la langue française, [s.d.], Banque de dépannage linguistique. Tiré de www.oqlf.gouv.qc.ca/ressources/bdl.html

\section{Ressources universitaires et collégiales}

Tous les établissements d'enseignement postsecondaires disposent de ressources et de services pour appuyer les étudiants dans leur apprentissage et pour les aider à avoir du succès dans leur carrière après l'obtention de leur diplôme. Vérifiez ce que votre établissement d'enseignement peut vous offrir. Vous trouverez ci-dessous quatre autres endroits où chercher :

Centre collégial de développement de matériel didactique, [s.d.], Amélioration du français. Tiré de www.ccdmd.qc.ca/fr

Cornell University - Learning Strategies Center : http://lsc.cornell.edu/Sidebars/ Study_Skills_Resources/SKResources. html

Kwantlen Polytechnic University - Essential Skills Resources: http://www. kpu.ca/learningcentres/resources

Stanford University - Resumes/CVs and Cover Letters : https://stu-dentaffairs. stanford. edu/cdc/resumes

Wilfrid Laurier University - Study Skills Webinars : http://www.wlu.ca/page. php?grp_id=1866\&p=12593

\section{Tutoriels d'aide à la rédaction sur YouTube}

«How to Write an Effective Essay»: https://www.youtube.com/watch?v= nWqMQ26Gqi4

« How to Write an Effective Essay: The Introduction » : https://www.youtube. com/watch?v=IN6IOSMviS4

«How to Write an Essay » : https://www.youtube.com/watch?v=liyFKU FCQno

\section{Sites Web canadiens affichant des emplois et des outils de carrière pour les jeunes}

Colombie-Britannique, WorkBC : http://www.workbc.ca/

Canada, Guichet-Emplois : https://www.guichetemplois.gc.ca/accueil-fra.do? lang=fra

Charity Village (pour ceux qui s'intéressent au secteur non marchand, c'està-dire aux organismes sans but lucratif) : http://charityvillage.com/

Ontario, Youth Employment Services : http://www.yes.on.ca

Québec, Emploi-Québec: http://www.emploiquebec.gouv.qc.ca/citoyens/profils/ jeune/ 


\section{Au sujet des auteurs}

Thomas Klassen est professeur au Département de science politique et à l'École de politiques et d'administration publiques de l'Université York. Il a obtenu son doctorat en sociologie de l'Université de Toronto alors qu'il travaillait à temps plein au gouvernement comme conseiller en politiques. Il a enseigné aux universités Ryerson et Trent, ainsi qu'en Allemagne et en Corée du Sud, où il a donné une variété de cours en sociologie, en études sociales, en science politique et en administration publique, y compris un cours du programme de MBA international (International Master of Business Administration) à la Schulich School of Business.

Auteur de plusieurs livres sur les politiques du milieu de travail, il a édité beaucoup d'autres ouvrages, y compris Casino State: Legalized Gambling in Canada. Son plus récent livre s'intitule Retirement in Canada. Il écrit souvent pour les médias de masse, comme le Toronto Star, le Globe and Mail et quelques autres.

Avant de devenir professeur, il a été pendant dix ans conseiller en politiques sociales et économiques et a passé une année à travailler pour une firme de consultants d'Ottawa. Au cours des dix dernières années, il a été consultant en problématiques liées aux milieux de travail dans des organismes communautaires, des cabinets d'avocats, des gouvernements provinciaux et fédéral, et des agences internationales.

En tant que personne qui bégaye (il n'a pas posé une seule question en classe pendant toutes ses études secondaires), il a également 
écrit sur l'expérience des personnes handicapées. Il fait actuellement partie d'un projet de développement de grande envergure, s'échelonnant sur plusieurs années et subventionné par le gouvernement du Canada, dont l'objectif est de créer de meilleurs débouchés pour les personnes handicapées au Bangladesh, en Inde et au Népal.

On peut souvent l'entendre marmonner « Personne ne m'écoute, personne ne m'écoute. Mes jumeaux de 8 ans ne m'écoutent pas, mes étudiants ne m'écoutent pas et ma femme ne m'écoute pas. » Il répète constamment les mêmes choses, ce qu'il attribue au fait qu'il est professeur, père de jumeaux et quelqu'un qui bégaye. Et au fait que personne ne l'écoute.

En 2014, il est déménagé en Corée du Sud pour deux ans avec sa famille parce que sa femme y avait été mutée par son employeur. Il a rédigé sa partie de ce livre à Séoul. Incapable de parler coréen, il remarque que personne ne porte attention quand il parle.

Il a récemment créé son propre site Web, dont il est très fier: www.thomasklassen.net.

Vous pouvez communiquer avec lui au tklassen@yorku.ca.

John A. Dwyer est professeur au Département des humanités de l'Université York à Toronto. Il a obtenu son doctorat en histoire des idées à l'Université de la Colombie-Britannique et a enseigné aux universités de la Colombie-Britannique et Simon Fraser, ainsi qu'au Collège North Island sur l'île de Vancouver. Il a édité de nombreuses collections, écrit beaucoup d'articles de journaux et est l'auteur de deux livres, l'un portant sur l'économiste et philosophe Adam Smith et l'autre, sur les Lumières écossaises du xviiie siècle. Son ouvrage intitulé Business History: Canada in the Global Community sert de manuel de cours dans les écoles de commerce.

En plus d'être professeur, il a travaillé pendant plusieurs années comme administrateur et consultant. Il a été directeur intérimaire du placement pour une importante école de commerce, où il aidait des diplômés à trouver des emplois au sein de compagnies. Il a également œuvré comme éducateur à distance pour les régions éloignées, collecteur de fonds pour une université, consultant en perfectionnement professionnel et directeur associé d'un centre d'enseignement universitaire. En tant que président de sa propre entreprise, il a fourni ses services de consultant à plusieurs universités et collèges.

Professeur talentueux, lauréat de plusieurs prix pour ses activités en salle de classe, il a pratiquement tout enseigné, des sciences 
naturelles à l'histoire, en passant par l'éthique et le commerce. Pendant plusieurs années, il a enseigné un cours de première année sur la pensée critique et analytique en affaires à la Schulich School of Business. Plus récemment, il a enseigné un cours intitulé «On Love » («Sur l'amour»).

Amateur de musique rock, ses musiciens préférés sont, entre autres, Joy Division, Radiohead, Sigur Rós, Pearl Jam, Nirvana, Dead Can Dance, Peter Gabriel, Björk, Richard Thompson et, surtout, Pere Ubu. Ses préférences plus obscures incluent Art Bears, Soft Machine, After Dinner et Honeymoon Killers. Il aime la nourriture la plus exotique et épicée possible, tant que ce n'est pas du poisson. Il déteste le poisson, en dépit de toutes les tentatives faites par sa blonde (comme elle aime qu'on l'appelle) d'en cacher dans ses ragoûts. Mais ce qu'il apprécie par-dessus tout, c'est le vin, le brandy et le whisky pur malt (chers, de préférence). Il pourra tout de même se contenter de bière s'il n'y a rien d'autre.

À la publication de ce livre, il sera près de la retraite. En tant qu'enfant de parents hippies des années 1960, il se souvient de certaines choses de la période psychédélique et demeure amer quant à la publicité mensongère de l'époque au sujet de "l'amour libre». Puisqu'il a une fille d'âge adulte, née d'un mariage antérieur, il aime prétendre qu'il comprend les jeunes d'aujourd'hui.

Vous pouvez communiquer avec lui au jdwyer@yorku.ca. 
Page blanche conservée intentionnellement 
Page blanche conservée intentionnellement 
Poursuivre ses études, c'est palpitant, mais c'est également stressant. Quels cours devrais-je suivre? Quel programme devrais-je choisir? Vais-je décrocher un emploi après avoir obtenu mon diplôme?

Rassurez-vous: ce livre permet de constater que la meilleure façon de bien préparer son entrée dans le marché du travail, c'est de réussir ses études. Travailler en équipe, respecter des échéanciers, relever des défis, savoir manier la plume et interagir avec des collègues: voilà autant d'éléments essentiels à tout emploi professionnel, et ces mêmes habiletés constituent justement les fondements de tout bon étudiant.

Que vous veniez de commencer vos études postsecondaires, que vous vous trouviez au beau milieu de celles-ci ou que vous soyez sur le point d'obtenir votre diplôme, ce guide pratique vous montre comment maîtriser ces compétences clés ainsi que les stratégies qui mènent à la réussite. Il s'adresse à tous les étudiants, qu'ils soient à l'université, au collège, au cégep ou au secondaire, ainsi qu'à leurs parents. Ce livre vous aidera à vous épanouir sur les plans scolaire, professionnel et personnel, l'objectif ultime étant d'atteindre le bonheur duquel découle une vie réfléchie, équilibrée et sur laquelle vous avez le contrôle.

« Dans un monde parfait, votre emploi idéal vous attendrait dès votre cérémonie de collation des grades terminée. Illusoire? Pas du tout! Avec un peu de planification, cela pourrait très bien vous arriver. "

Thomas R. Klassen est professeur au Département de science politique et à l'École d'administration et de politiques publiques à l'Université York.

John A. Dwyer est professeur au Département des humanités de I'Université York.

Émilie Laramée est traductrice-réviseure à l'Université d'Ottawa.

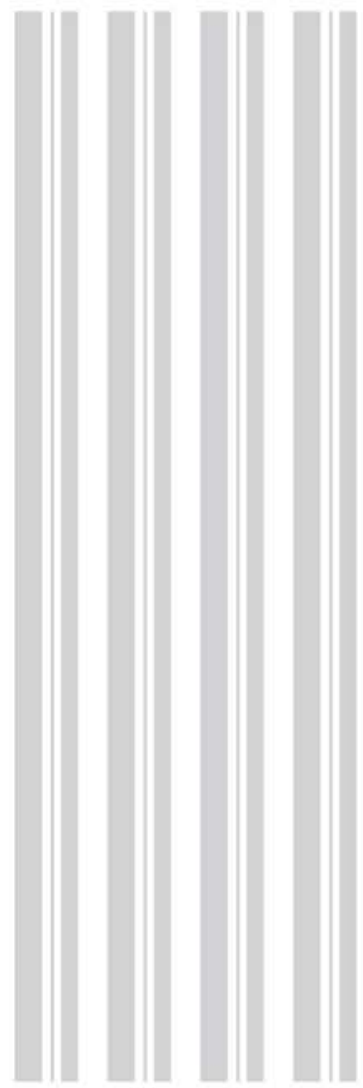

